\title{
De novo design, synthesis, and mechanistic evaluation of short peptides that mimic heat shock protein 27 activity
}

\author{
Jessica Khoa , P. Chi Phamª, Suhyeon Kwon ${ }^{a}$, Alana Y. Huanga, Joel P. Rivers ${ }^{a}$, Huixin \\ Wang $^{a}$, Heath Ecroyd ${ }^{b}$, W. Alexander Donald ${ }^{a}$, and Shelli R. McAlpine *c \\ aDepartment of Chemistry, University of New South Wales, Sydney NSW 2052 Australia. \\ ' School of Biological Sciences, University of Wollongong, Wollongong, NSW, 2522, Australia \\ c Department of Chemistry, University of California, Irvine CA, USA \\ *Corresponding author email: Mcalpine@UCl.edu
}

De novo design, synthesis and development of micro-chaperones that inhibit citrate synthase aggregation based on the $\mathrm{N}$-terminus of heat shock protein 27 (Hsp27). ........................................... S1

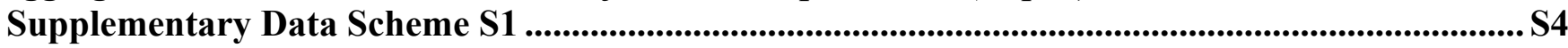

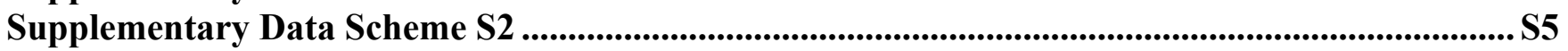

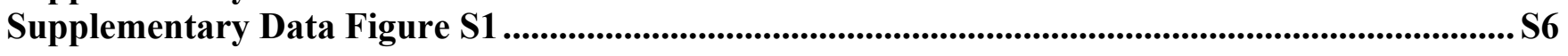

Supplementary Data Figure S2 ................................................................................................................. S7

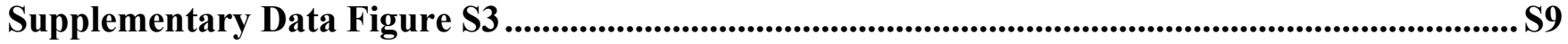

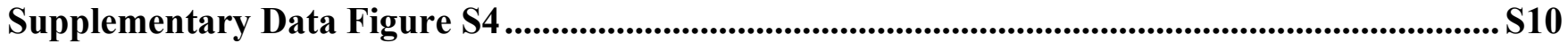

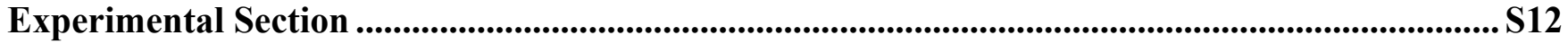

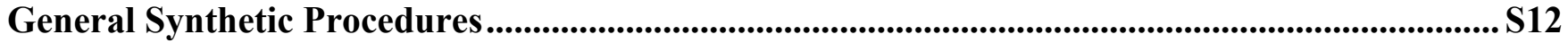

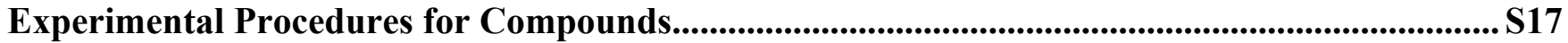

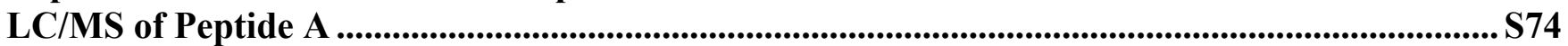

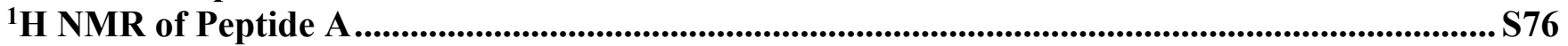

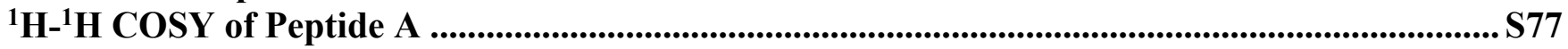

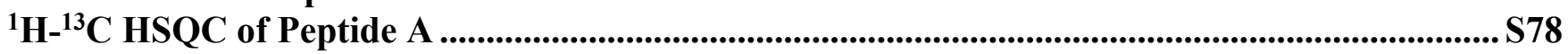

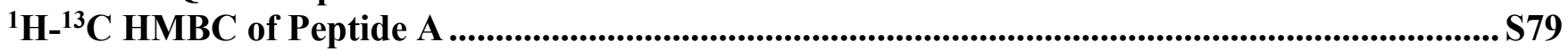

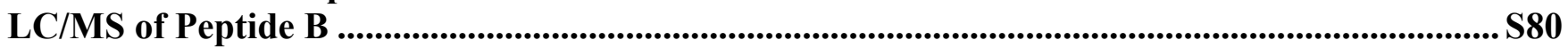

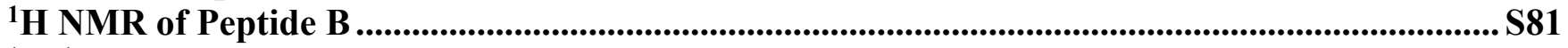

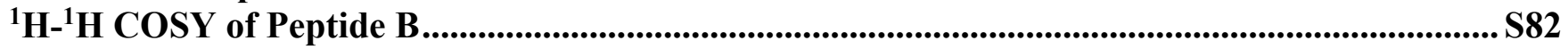

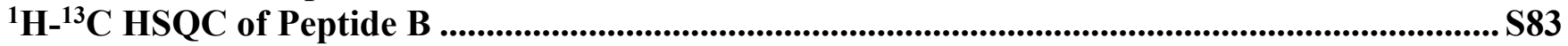

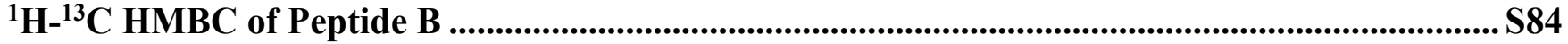

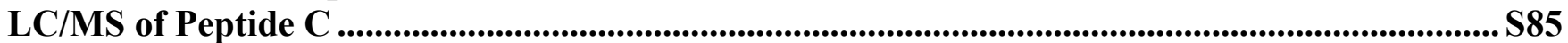

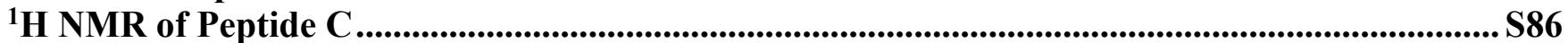

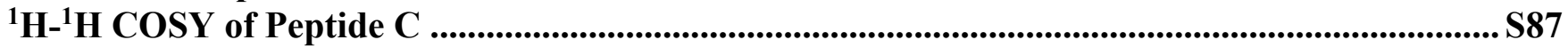

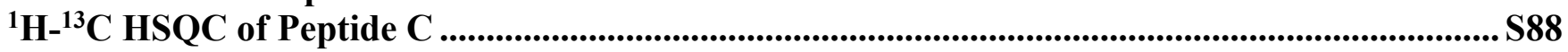

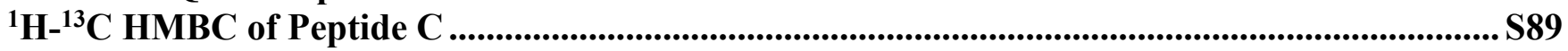

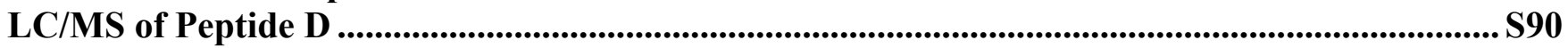

${ }^{1}$ H NMR of Peptide D ............................................................................................................................................ S91

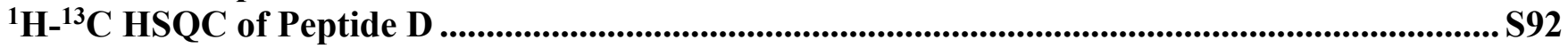

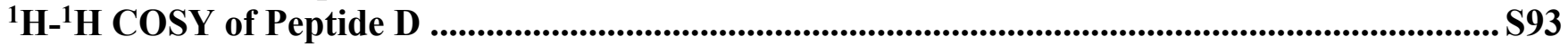

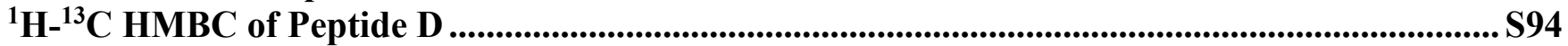

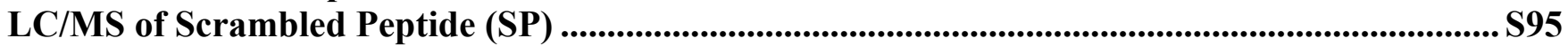

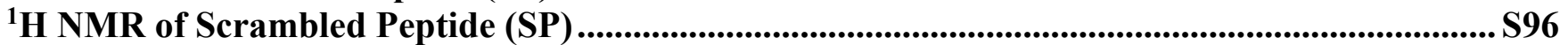

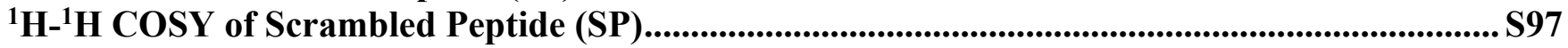

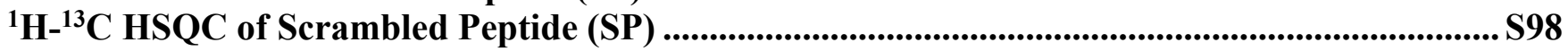

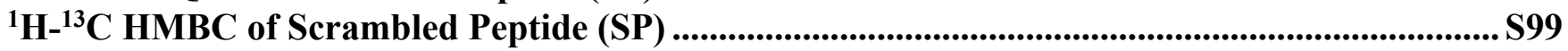

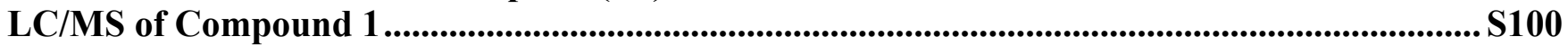

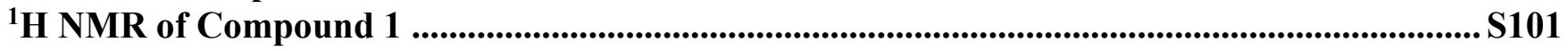




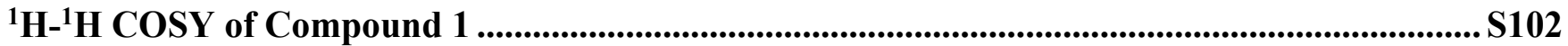

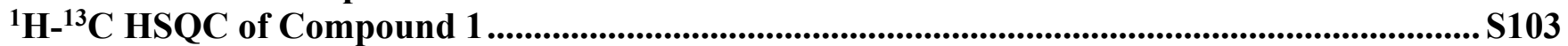

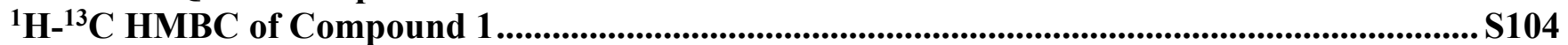

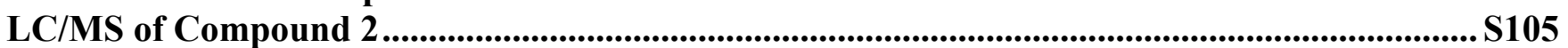

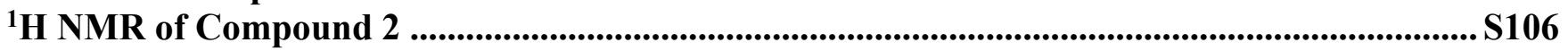

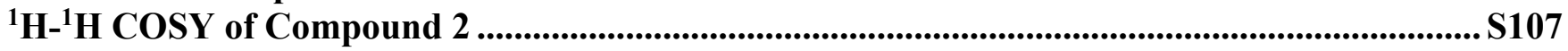

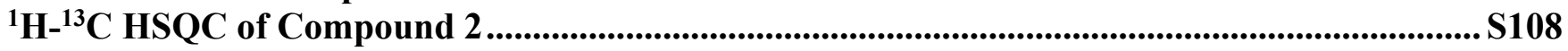

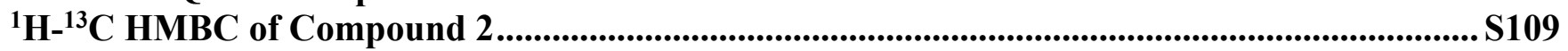

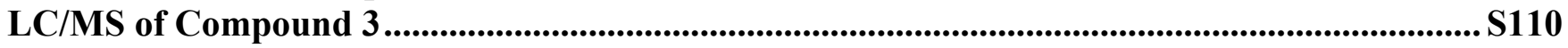

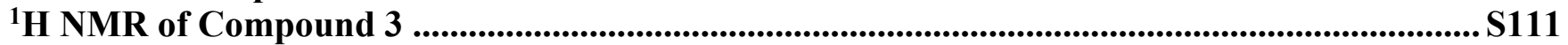

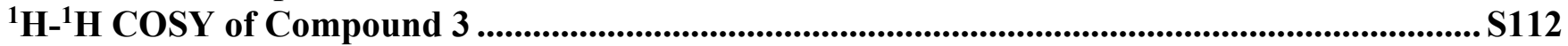

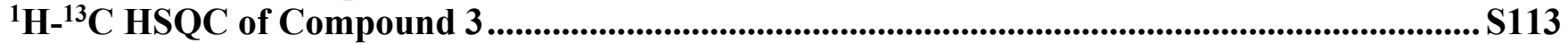

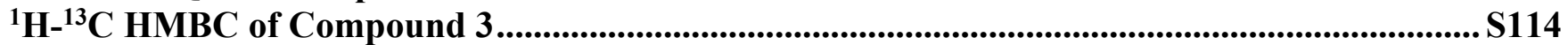

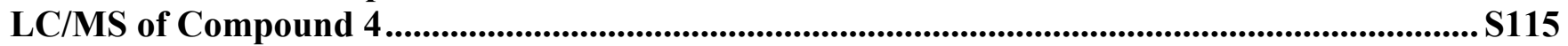

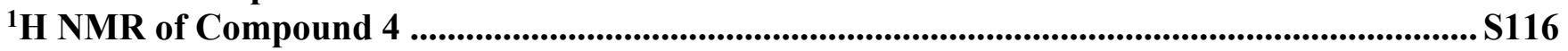

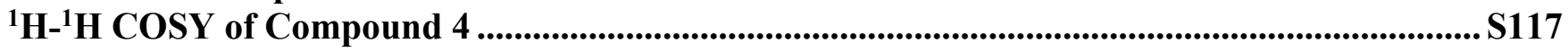

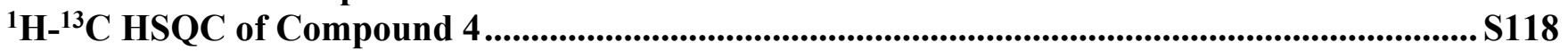

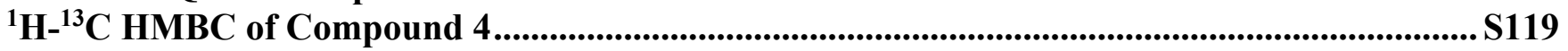

LC/MS of Compound 5 ...................................................................................................................................S120

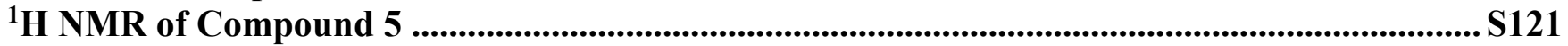

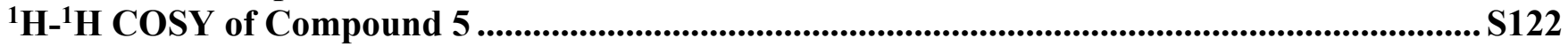

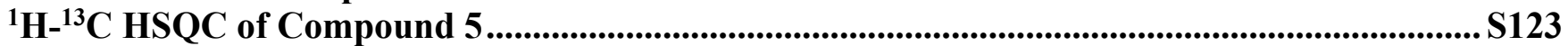

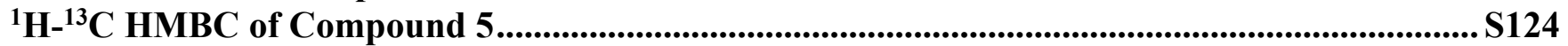

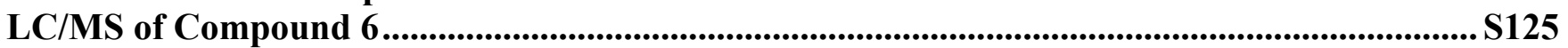

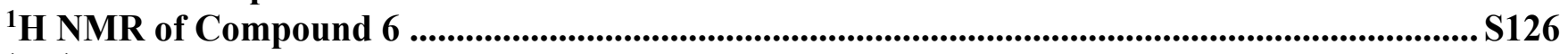

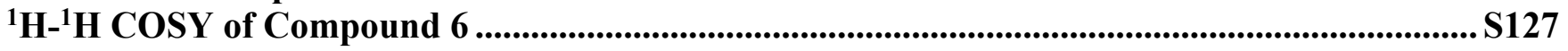

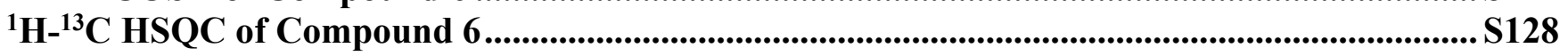

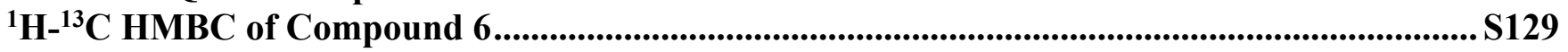

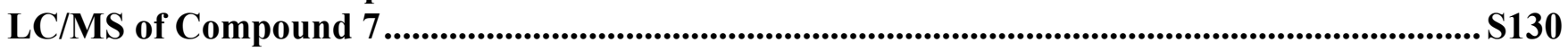

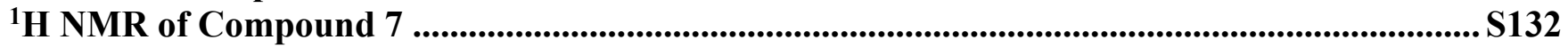

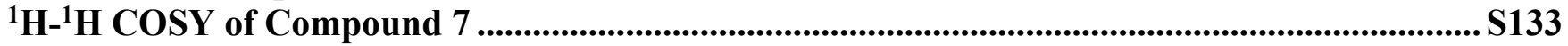

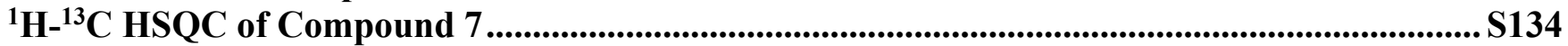

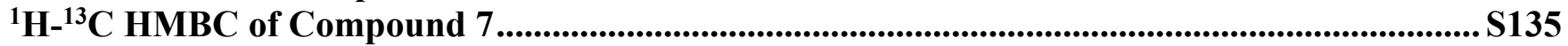

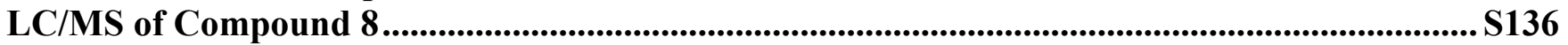

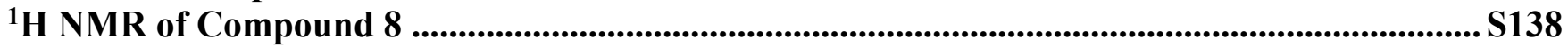

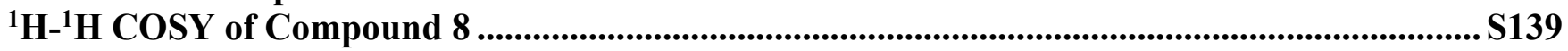

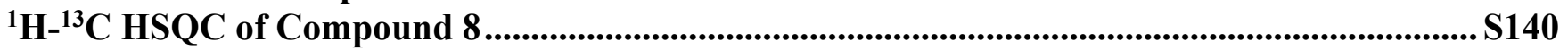

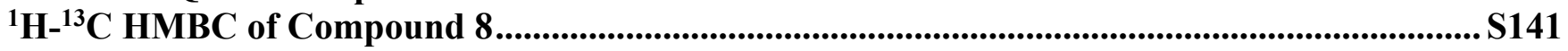

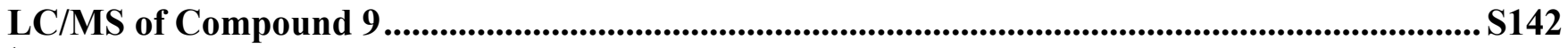

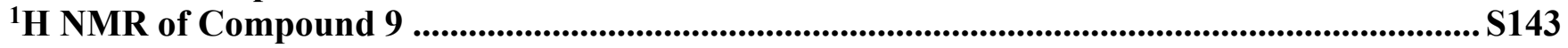

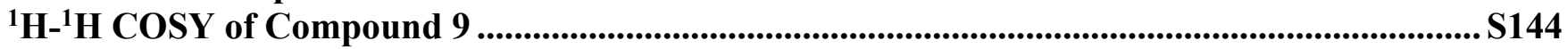

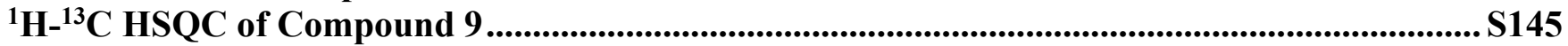

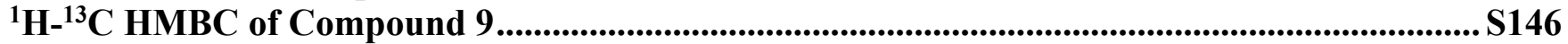

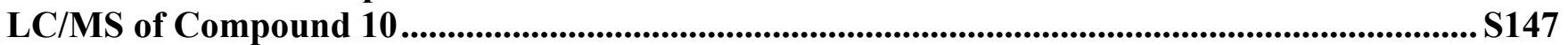

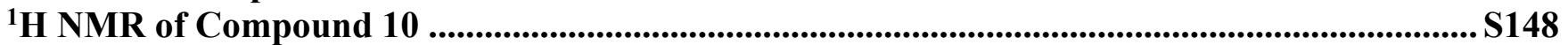

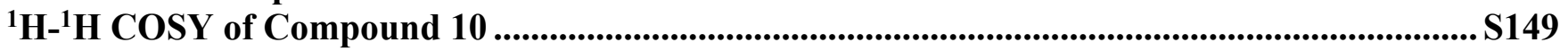

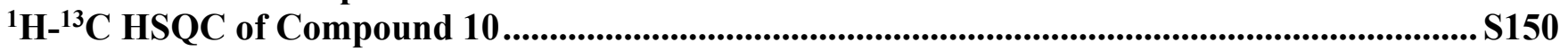

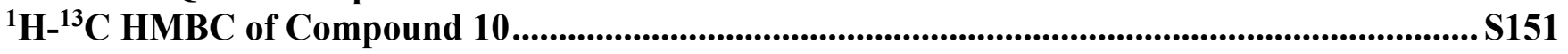

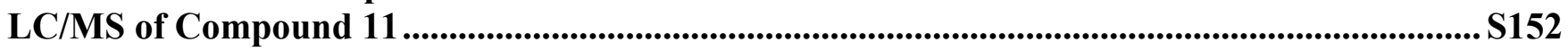

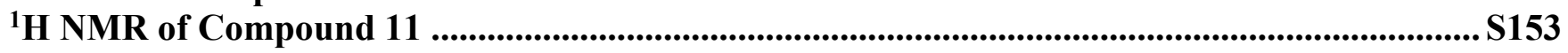

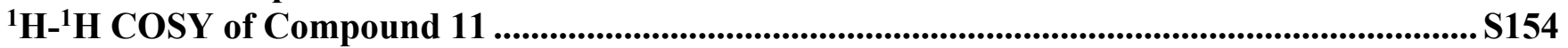




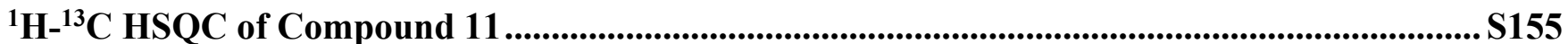

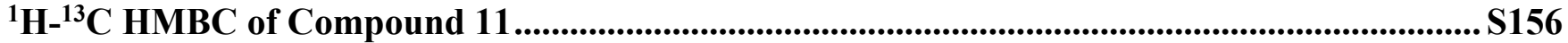

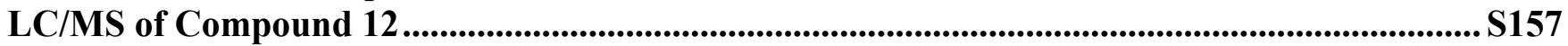

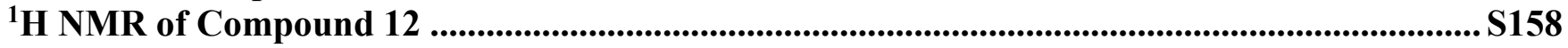

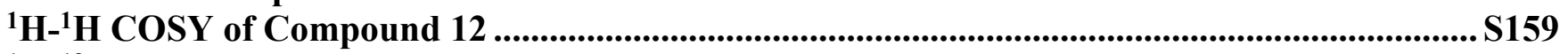

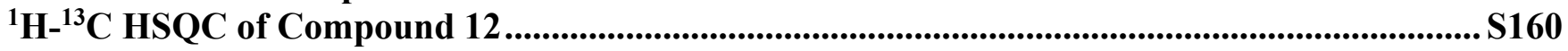

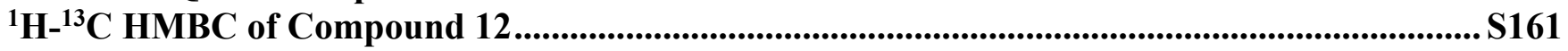

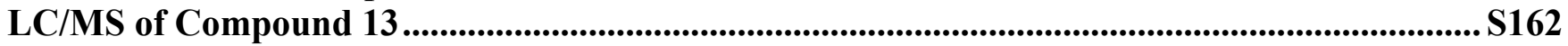

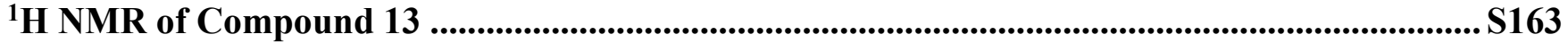

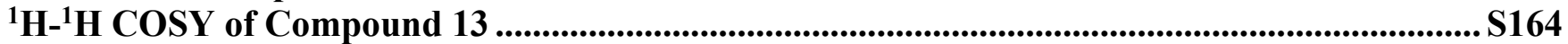

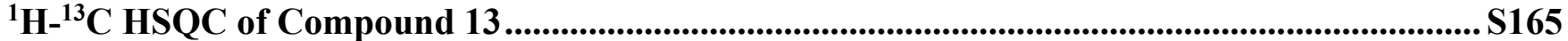

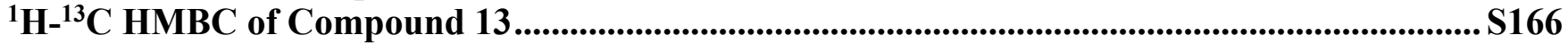




\section{Supplementary Data Scheme S1}
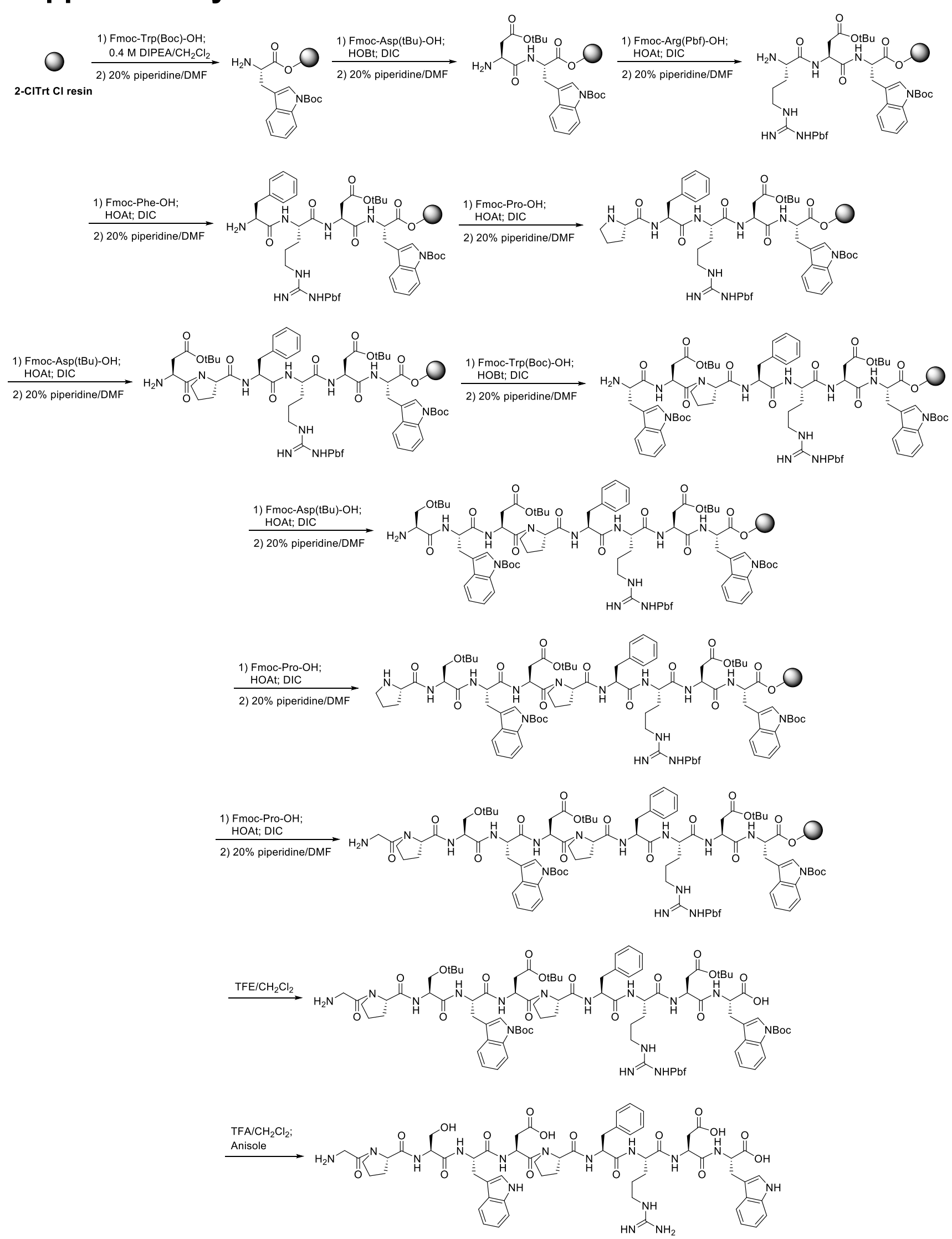

Scheme S1. Synthetic scheme of Peptide A. Resin loading was done using Fmoc-Trp(Boc)-OH (3 equiv.) and $0.4 \mathrm{M}$ DIPEA $/ \mathrm{CH}_{2} \mathrm{Cl}_{2}$. Subsequent peptide coupling were completed using their respective Fmoc-protected amino acids (2 equiv.), HOAt or HOBt (2 equiv.), DIC (4 equiv.) in 
DMF (0.3 M). Fmoc-protecting groups were removed using $20 \%$ piperidine/DMF. Resin cleavage was completed using TFE: $\mathrm{CH}_{2} \mathrm{Cl}_{2}(1: 1,10 \mathrm{~mL} / \mathrm{g})$ and the protecting groups were removed using TFA ( $4 \mathrm{~mL} / \mathrm{g}$ of peptide with anisole (2 equiv./side chain protecting group).

\section{Supplementary Data Scheme S2}

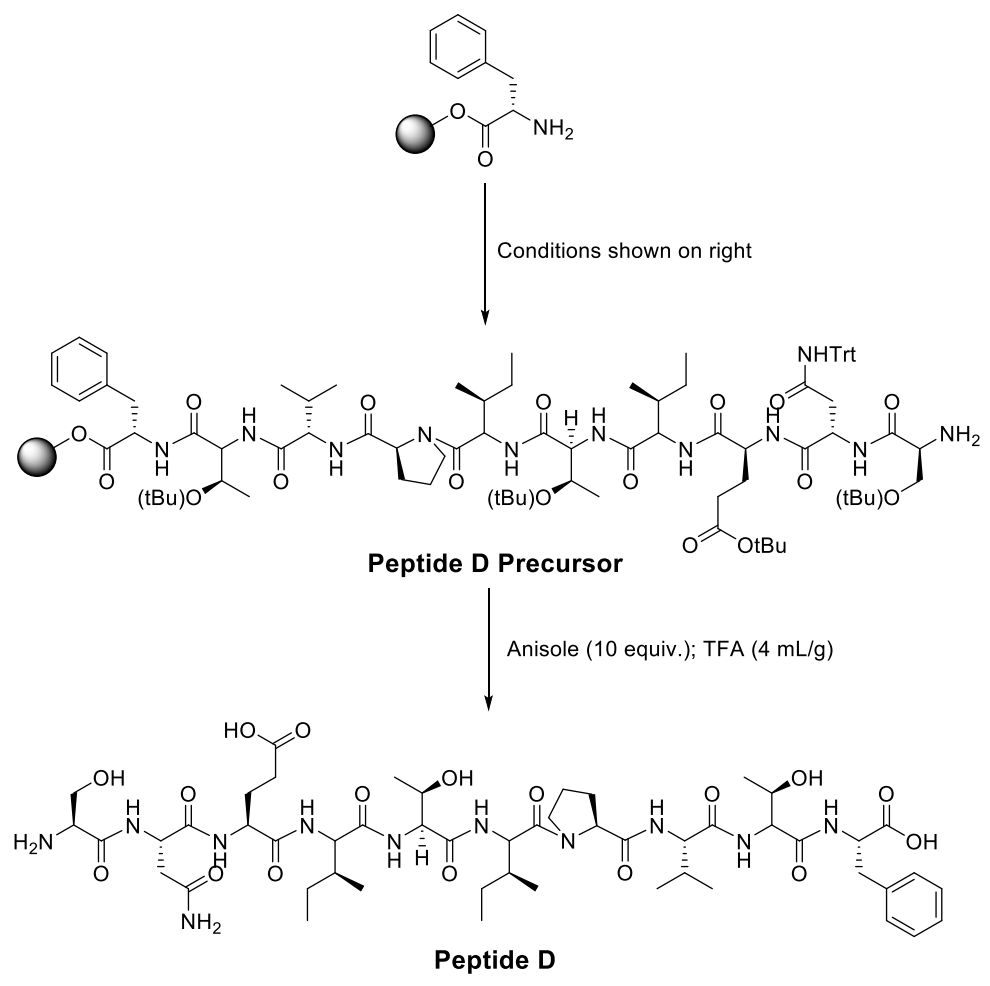

\section{Coupling Conditions}

1. (a) Fmoc-Thr(tBu)-OH (3 equiv.); HOBt (3 equiv); DIC (6 equiv.); in DMF $(0.2 \mathrm{M})$

(b) $20 \%$ piperidine in DMF

2. (a) Fmoc-Val-OH (3 equiv.); HOBt (3 equiv); DIC (6 equiv.); in DMF ( $0.2 \mathrm{M})$

(b) $20 \%$ piperidine in DMF

3. (a) Fmoc-Pro-OH (3 equiv.); HOAt (3 equiv); DIC (6 equiv.); in DMF $(0.2 \mathrm{M})$

(b) $20 \%$ piperidine in DMF

4. (a) Fmoc-lle-OH (3 equiv.); HOAt (3 equiv); DIC (6 equiv.); in DMF ( $0.2 \mathrm{M})$

(b) $20 \%$ piperidine in DMF

5. (a) Fmoc-Thr(tBu)-OH (3 equiv.); HOBt (3 equiv); DIC (6 equiv.); in DMF ( $0.2 \mathrm{M})$

(b) $20 \%$ piperidine in DMF

6. (a) Fmoc-lle-OH (3 equiv.); HOBt (3 equiv); DIC (6 equiv.); in DMF (0.2 M)

(b) $20 \%$ piperidine in DMF

7. (a) Fmoc-Glu(tBu)-OH (3 equiv.); HOBt (3 equiv); DIC (6 equiv.); in DMF ( $0.2 \mathrm{M})$

(b) $20 \%$ piperidine in DMF

8. (a) Fmoc-Asn(Trt)-OH (3 equiv.); HOAt (3 equiv); DIC (6 equiv.); in DMF ( $0.2 \mathrm{M})$

(b) $20 \%$ piperidine in DMF

9. (a) Fmoc-Ser(tBu)-OH (3 equiv.); HOAt (3 equiv); DIC (6 equiv.); in DMF ( $0.2 \mathrm{M})$

(b) $20 \%$ piperidine in DMF

Scheme S2. Synthetic scheme of Peptide D using a Biotage Initiator + Alstra Automated Microwave Peptide Synthesiser. Using pre-loaded H-Phe-2-CITrt resin, subsequent peptide couplings were completed using their respective Fmoc-protected amino acids (3 equiv.), HOAt or HOBt (3 equiv.), DIC (6 equiv.) in DMF $(0.2 \mathrm{M})$ at $70{ }^{\circ} \mathrm{C}$. Fmoc-protecting groups were removed using $20 \%$ piperidine/DMF. Resin cleavage was manually completed using TFE: $\mathrm{CH}_{2} \mathrm{Cl}_{2}(1: 1,10 \mathrm{~mL} / \mathrm{g})$ and the protecting groups were removed using TFA $(4 \mathrm{~mL} / \mathrm{g}$ of peptide with anisole ( 2 equiv./side chain protecting group). 


\section{Supplementary Data Figure S1}

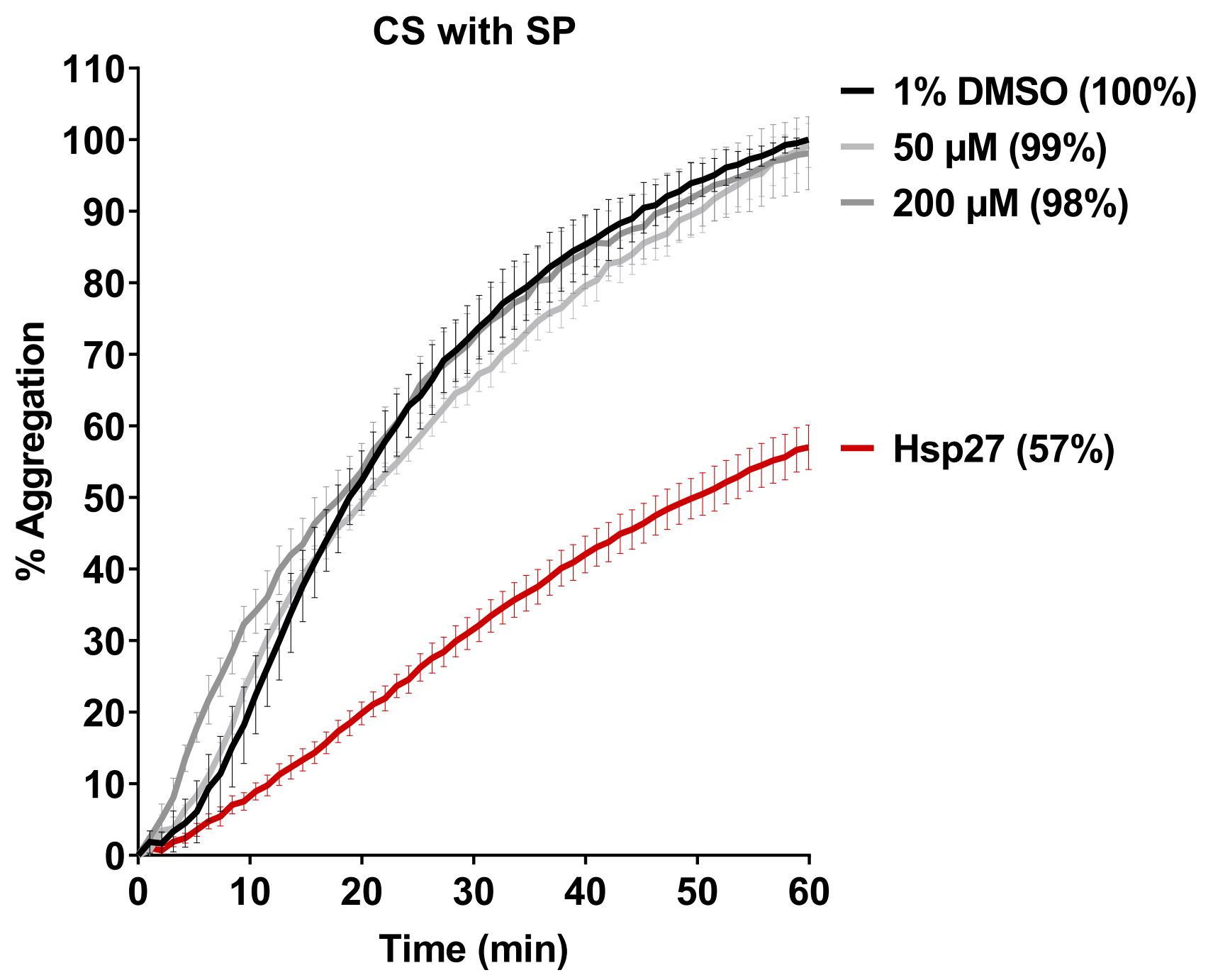

Figure S1. Effects of the Scrambled Peptide (SP) on CS aggregation, as monitored by light scattering at $360 \mathrm{~nm}$. CS $(150 \mathrm{nM})$ was heated at $43^{\circ} \mathrm{C}$ for 1 hour in the presence of 50 and 200 $\mu \mathrm{M}$ of SP. Aggregation of CS with $1 \%$ DMSO was set to $100 \%$ and Hsp27 (600 nM) reflects the impact of the chaperone on CS on the day the experiments were run. All data and error bars represent mean \pm S.E.M. for at least three independent experiments. 


\section{Supplementary Data Figure S2}

(a)

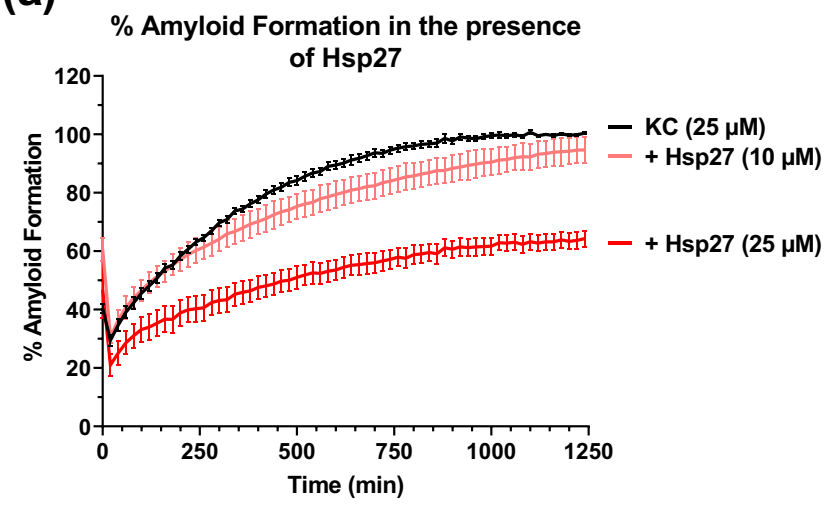

(c)

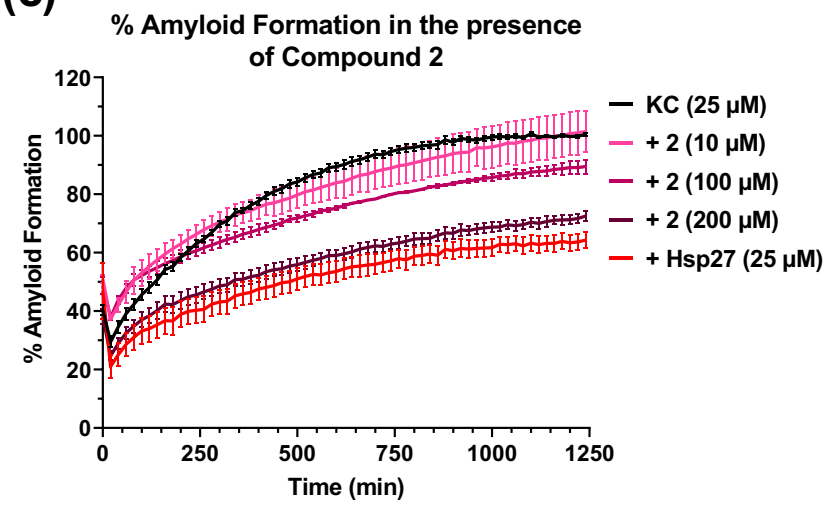

(b)

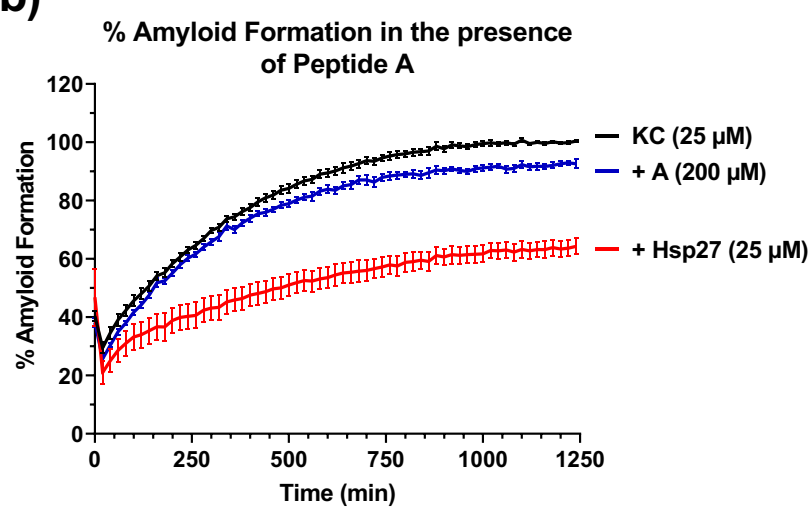

(d)

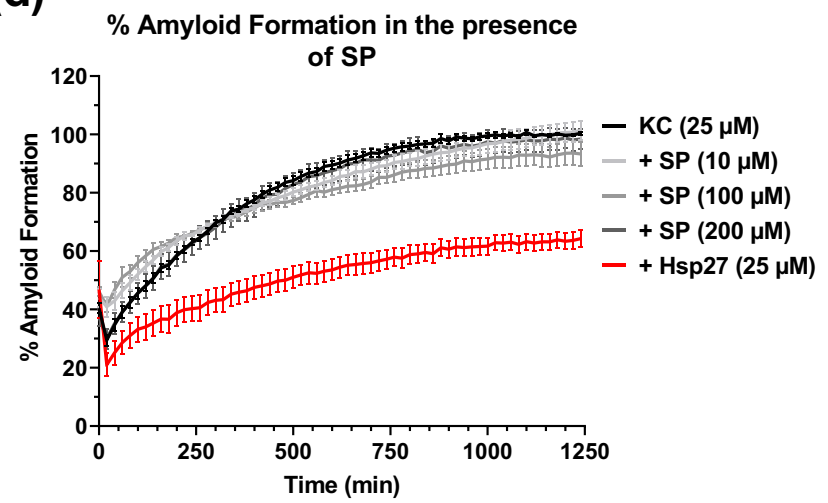

(e)

\section{$\%$ Amyloid Formation in the presence of Hsp27 and Peptides A, 2, and SP}

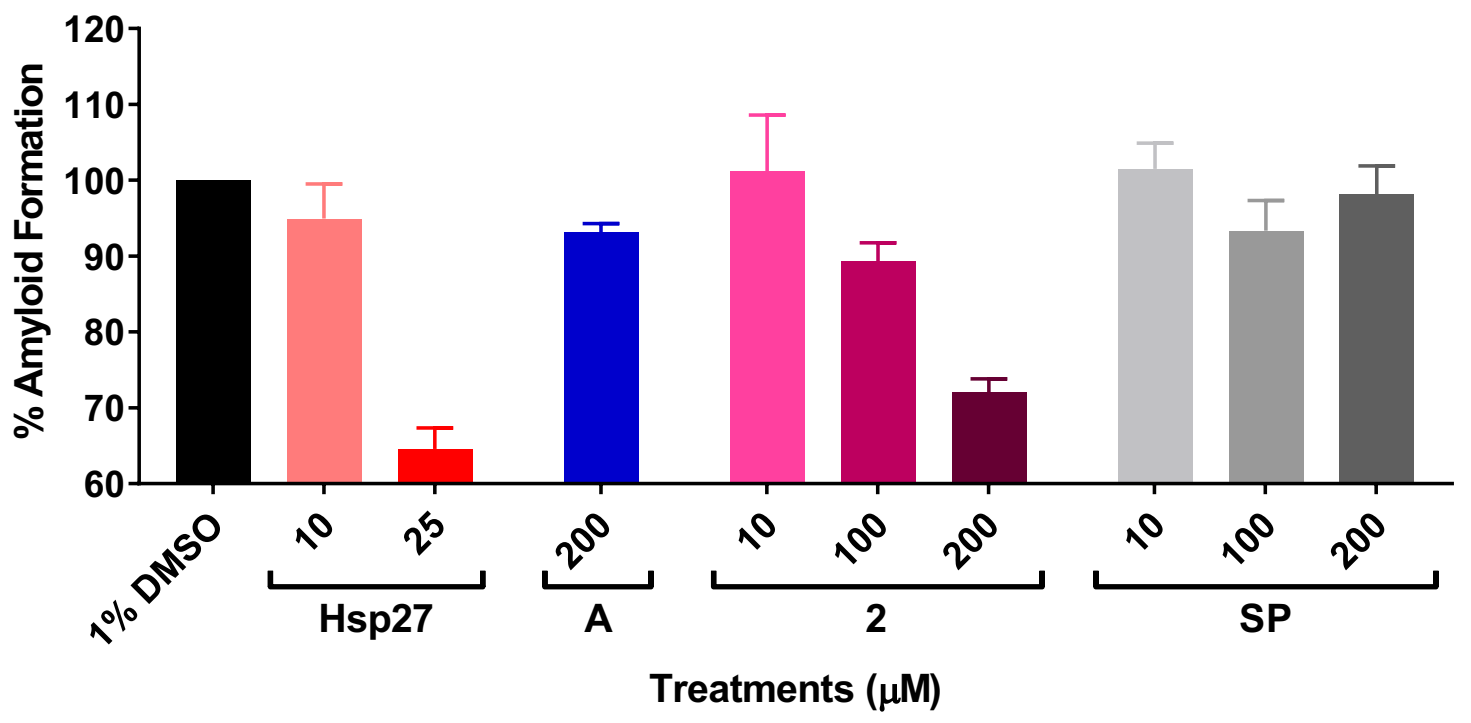

Figure S2. Effect of Hsp27 and Peptides A, 2 and SP on amyloid formation via K-casein assay. K-casein $\left(25 \mu \mathrm{M}\right.$ ) was incubated at $37^{\circ} \mathrm{C}$ for $\sim 21$ hours in the presence of (a) Hsp27 (10 or 25 $\mu \mathrm{M})$ or (b) Peptides A (200 $\mu \mathrm{M})$, (c) $2(10,100$ or $200 \mu \mathrm{M})$, or (d) SP $(10,100$ or $200 \mu \mathrm{M})$ in 50 $\mathrm{mM}$ phosphate buffer $(\mathrm{pH} 7)$. A control with 1\% DMSO was used, where amyloid formation in this sample was set to $100 \%$. Amyloid formation was initiated using DTT (20 mM) and detected 
via ThT $(20 \mu \mathrm{M})$ fluorescence. Excitation and emission wavelengths were 440 and $480 \mathrm{~nm}$ respectively. All data and error bars represent mean \pm S.E.M. for at least three independent experiments. (e) Summary of data where the endpoints of each trace are plotted. 
CS with Hsp27 vs Hsp27-3D

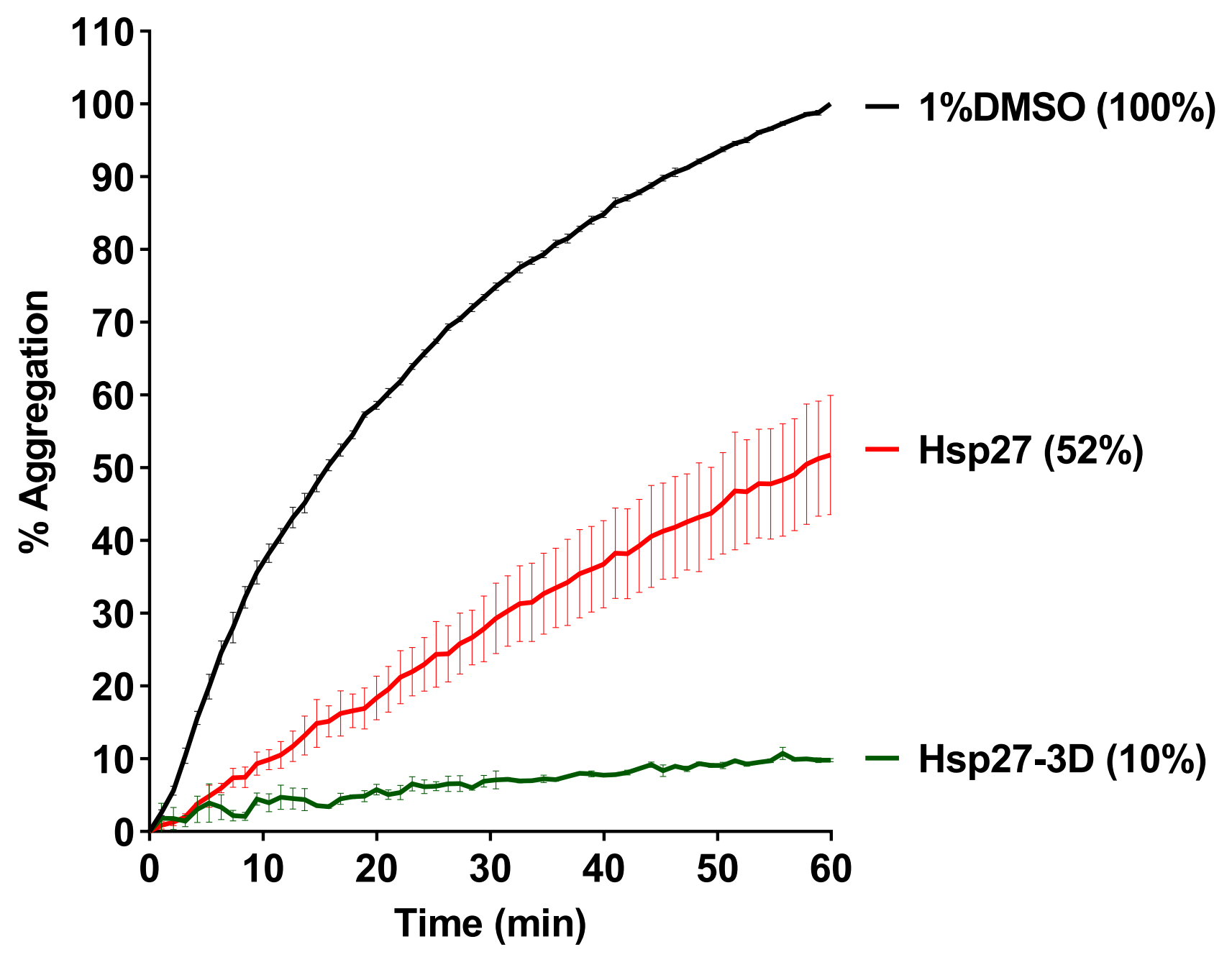

Figure S3. Effect of Hsp27-3D on CS aggregation, as monitored by light scattering at $360 \mathrm{~nm}$. $\mathrm{CS}$ was heated at $43^{\circ} \mathrm{C}$ for 1 hour in the presence of Hsp27-3D (600 nM). Aggregation of CS was set to $100 \%$ and Hsp27 (600 nM) reflects the impact of the chaperone on CS on the day the experiments were run. All data and error bars represent mean \pm S.E.M. for at least two independent experiments. 


\section{Supplementary Data Figure S4}

\section{CS with Compound 13}

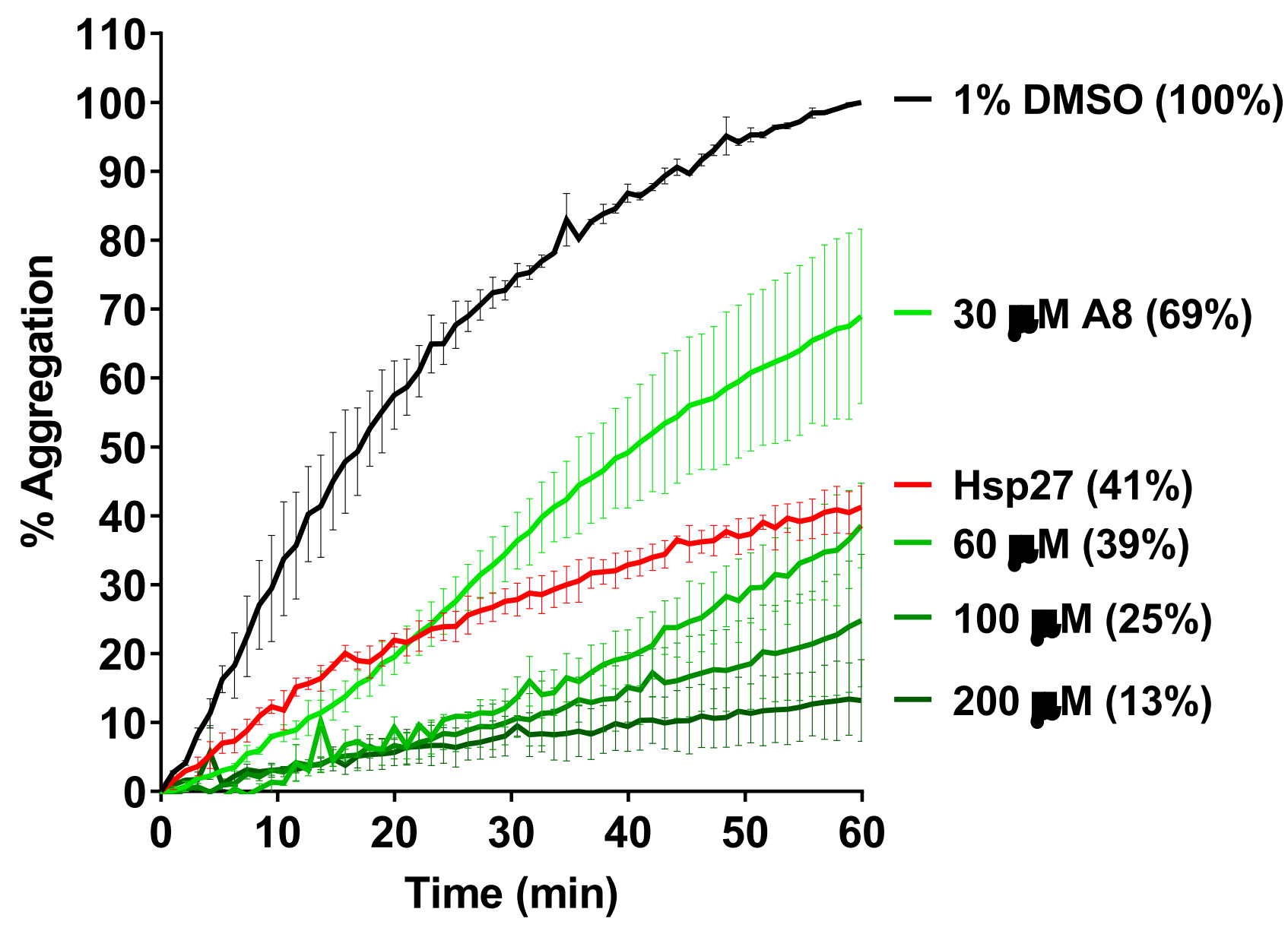

Figure S4. Effects of compound 13 on CS aggregation at multiple concentrations, as monitored by light scattering at $360 \mathrm{~nm}$. CS $(150 \mathrm{nM})$ was heated at $43^{\circ} \mathrm{C}$ for 1 hour in the presence of 50 and $200 \mu \mathrm{M}$ of SP. Aggregation of CS with 1\% DMSO was set to $100 \%$ and Hsp27 (600 nM) reflects the impact of the chaperone on CS on the day the experiments were run. All data and error bars represent mean \pm S.E.M. for at least two independent experiments 


\section{Experimental Section}

\section{General Remarks}

All chemicals were purchased from commercial suppliers (Chem-Impex International and Sigma Aldrich) and used without further purification. All moisture sensitive reactions were performed using anhydrous solvents under nitrogen gas. Removal of solvent was carried out under reduced pressure using a Buchi R210 rotary evaporator.

Semi-preparative HPLC for purification was performed using a GRACE VisionHT C18 column $(5 \mu \mathrm{m}, 22 \times 150 \mathrm{~mm})$ or a Phenomenex Aeris XB-C18 column $(5 \mu \mathrm{m}, 21.2 \times 150 \mathrm{~mm})$ on a Shimadzu Prominence High Performance LCMS 2010EV system. The mobile phase consisted of milli-Q water with $0.1 \%(\mathrm{v} / \mathrm{v})$ formic acid (Mobile Phase A), and HPLC grade acetonitrile with $0.1 \%(\mathrm{v} / \mathrm{v})$ formic acid (Mobile Phase B) at a flow rate of $5 \mathrm{~mL} / \mathrm{min}$, starting at 95\% Mobile Phase A and 5\% Mobile Phase B.

LC/MS analyses were performed using a Phenomenex Aeris XB-C18 column $(3.6 \mu \mathrm{m}, 2.1$ x 100 mm) on either a Shimadzu LCMS 2020 or Shimadzu LCMS 8030. The mobile phase consisted of milliQ water with $0.1 \%(\mathrm{v} / \mathrm{v})$ formic acid (Mobile Phase A), and HPLC grade acetonitrile with $0.1 \%(\mathrm{v} / \mathrm{v})$ formic acid (Mobile Phase B) at a flow rate of $0.2 \mathrm{~mL} / \mathrm{min}$, starting at 95\% Mobile Phase A and 5\% Mobile Phase B.

${ }^{1} \mathrm{H}$ and ${ }^{13} \mathrm{C}$ NMR spectra were obtained on Bruker Avance III $600 \mathrm{MHz}$. Multiplicity of NMR signals were represented by the following abbreviations: $\mathrm{s}=$ singlet, $\mathrm{d}=$ doublet, $\mathrm{t}=$ triplet, $\mathrm{q}=$ quartet, quin $=$ quintet $\mathrm{m}=$ multiplet, $\mathrm{dd}=$ doublet of doublet. Assignment of resonances for each residue was accomplished using 1H, HSQC, HMBC and COSY spectra.

\section{General Synthetic Procedures}

\section{Solid-Phase Peptide Synthesis}

Stepwise SPPS was performed in a polypropylene solid-phase extraction cartridge fitted with a $20 \mu \mathrm{m}$ polyethylene frit purchased from Applied Separations (Allentown, PA). 


\section{Resin Loading}

The resin was weighed, transferred to the cartridge and swelled in $\mathrm{CH}_{2} \mathrm{Cl}_{2}$ for 30 mins prior to the resin loading reaction. The appropriate Fmoc-protected amino acid was dissolved in the minimum amount of 0.4M DIPEA in $\mathrm{CH}_{2} \mathrm{Cl}_{2}$. The swelled resin was then drained, and the dissolved amino acid was added to resin in the cartridge. The suspension was agitated for a minimum of 3 hours at room temperature. The resin was washed 3 times with $\mathrm{CH}_{2} \mathrm{Cl}_{2}, 3$ times with DMF and 3 times with $\mathrm{CH}_{2} \mathrm{Cl}_{2}$. The resin was then dried in vacuo overnight. A $~ 5 \mathrm{mg}$ sample of resin was used to determine the amino acid loading. $20 \%$ piperidine in DMF was added to the sample to cleave the Fmoc protecting group. The resin was filtered away and the remaining solution was diluted 1 in 20 and the UV absorbance measured at $301 \mathrm{~nm}$ using a Cary 50 Bio UV-Vis instrument. DMF was used as a blank and samples were measured in a $1 \mathrm{~mL}$ quartz cuvette. The resin loading was then determined using the following formula:

Resin loading (mmol/g)

$=($ Abs $\mathrm{x}$ cleavage volume $\mathrm{x}$ dilution factor $) /($ extinction coefficient $\mathrm{x}$ cuvette width $\mathrm{x}$ resin mass $)$

$=($ Abs $\times 1 \mathrm{~mL} \times 20) /\left(7800 \mathrm{~mL} \mathrm{mmol}^{-1} \mathrm{~cm}^{-1} \times 1 \mathrm{~cm}^{\circ}\right.$ resin mass in $\left.\mathrm{g}\right)$

$=($ Abs $\times 20 \mathrm{~mL}) /\left(7800 \mathrm{~mL} \mathrm{mmol}^{-1} \mathrm{x}\right.$ resin mass in $\left.\mathrm{g}\right)$

\section{Coupling Reaction}

Prior to each coupling reaction, the resin was swelled in DMF for $0.5-1 \mathrm{hr}$, then the DMF was drained. Couplings were performed in DMF at a concentration of $0.3 \mathrm{M}$. Fmoc-protected amino acid (2 equiv.) and either 1-Hydroxybenzotriazole hydrate (HOBt) or 1-Hydroxy-7-azabenzotriazole HOAt (2 equiv.) were mixed with the resin. N, N'-Diisopropylcarbodiimide (DIC) (4 equiv.) was then added to activate the reaction. Coupling reaction was run for a minimum of 4 hours while shaking (Labquake tube shaker, Thermo Fisher Scientific) at room temperature. A negative ninhydrin test was used to confirm reaction completion. Once completed, the reaction mixture was drained, and the resin was subjected to Fmoc Removal. 


\section{Fmoc Removal}

The Fmoc protecting group was removed using the following washes: DMF ( 3 x $1 \mathrm{~min}$ ), 20\% piperidine in DMF (1 x $5 \mathrm{~min}), 20 \%$ piperidine in DMF (1 x $10 \mathrm{~min}), \mathrm{DMF}(2 \times 1 \mathrm{~min})$, $i \operatorname{PrOH}(1 \times 1 \mathrm{~min}), \mathrm{DMF}(1 \times 1 \mathrm{~min}), i \operatorname{PrOH}(1 \times 1 \mathrm{~min})$ and $\mathrm{DMF}(3 \times 1 \mathrm{~min})$. The resin was then ready for the next coupling reaction.

\section{Resin Cleavage of Linear Peptide}

Once the desired peptide was generated, the final Fmoc protecting group was removed following Fmoc Removal procedure with the following additional washes: $\mathrm{DMF}(3 \times 1 \mathrm{~min}), i \mathrm{PrOH}(3 \times 1 \mathrm{~min})$ and $\mathrm{MeOH}$ ( $3 \times 1 \mathrm{~min}$ ). The resin-bound peptide was then dried in vacuo overnight. The resin was then cleaved from the linear peptide using TFE and $\mathrm{CH}_{2} \mathrm{Cl}_{2}(1: 1 \mathrm{v} / \mathrm{v})$ at a concentration of $10 \mathrm{~mL} / \mathrm{g}$ resin, adding the $\mathrm{CH}_{2} \mathrm{Cl}_{2}$ first to swell the resin for 30 mins followed by TFE. The reaction mixture was stirred at room temperature for 48 hours before filtering the resin. The filtrate was concentrated and washed at least 10 times with $\mathrm{CH}_{2} \mathrm{Cl}_{2}$ to remove residual entrapped TFE. The product was then dried in vacuo overnight to produce the linear peptide.

\section{Side Chain Deprotection}

Amino acid side chain protecting groups were removed using TFA in $\mathrm{CH}_{2} \mathrm{Cl}_{2}(9: 1 \mathrm{v} / \mathrm{v})$ with anisole or EDT (at least 2 equiv. per protecting group). Anisole or EDT was added to the peptide, whilst stirring, followed by the TFA solution at a concentration of $4 \mathrm{~mL} / \mathrm{g}$ compound. The reaction was left stirring at room temperature overnight. The reaction was monitored using LC/MS and once complete the reaction solution was dried under a stream of nitrogen before redissolving in $\mathrm{CH}_{2} \mathrm{Cl}_{2}$ and evaporating multiple times to remove residual entrapped TFA. The product was precipitated using diethyl ether, collected via centrifugation and lyophilised to produce the crude linear peptide. 


\section{Biology Methods}

\section{Heat denatured citrate synthase aggregation assay}

An ammonium sulfate suspension of CS from porcine heart (Sigma-Aldirch, C3260) was centrifuged at $15,000 \mathrm{x}$ g at $4^{\circ} \mathrm{C}$ for 10 mins. The supernatant is removed and the pellet is redissolved in TE buffer (50 $\mathrm{mM}$ Tris-base, $2 \mathrm{mM} \mathrm{Na}$-EDTA, $\mathrm{pH}$ 8.0). The concentration was determined using a Pierce ${ }^{\mathrm{TM}}$ BCA Protein Assay Kit (ThermoFisher, 23225), following the manufacturers protocol. The CS solution was then aliquoted and stored at $-80^{\circ} \mathrm{C}$. For each set of experiments, the $\mathrm{CS}$ was thawed and diluted to 30 $\mu \mathrm{M}$ (monomer) with TE buffer. The protein is kept on ice.

Hsp27 was diluted to $60 \mu \mathrm{M}$ using $40 \mathrm{mM}$ HEPES-KOH buffer (pH 7.5, filtered and degassed) and kept on ice. All test compounds were dissolved in 100\% DMSO and were diluted to a final concentration of $1 \%$ DMSO in each reaction.

All readings were taken on a Varian Cary Eclipse fluorometer at $43^{\circ} \mathrm{C}$. Excitation and emission wavelengths were both set to $360 \mathrm{~nm}$ and both excitation and emission slit widths were set to $2.5 \mathrm{~nm}$. Data points were recorded every $30 \mathrm{sec}$.

In a $400 \mathrm{uL} \mathrm{Hellma}{ }^{\circledR}$ Fluorescence Microcuvette (Sigma-Aldrich, Z802778), $390 \mu \mathrm{L}$ of $40 \mathrm{mM}$ HEPES-KOH buffer ( $\mathrm{pH} 7.5$, filtered and degassed) was pipetted into the cuvette followed by $4 \mu \mathrm{L}$ of Hsp27 or buffer, and $4 \mu \mathrm{L}$ of DMSO or test compound. The solution was mixed by pipetting before being incubated at $43^{\circ} \mathrm{C}$ for 5 mins. The light scattering is monitored for 5 min to observe any changes due to the Hsp27 and compounds. Once completed, CS is diluted 1:200 into the reaction mixture, stirred and incubated at $43^{\circ} \mathrm{C}$ for $3 \mathrm{~min}$ before monitoring light scattering for $60 \mathrm{~min}$.

\section{Chemically denatured citrate synthase aggregation assay}

An ammonium sulfate suspension of CS from porcine heart (Sigma-Aldrich, C3260) was incubated in denaturing buffer (6.6 M GdnCl, 5.5 mM DTT) at concentration of $18.7 \mu \mathrm{M}$ for $1 \mathrm{hr}$ at room temperature. Hsp27 was diluted to $60 \mu \mathrm{M}$ using Refolding buffer ( $25 \mathrm{mM}$ Tris-base, $100 \mathrm{mM} \mathrm{NaCl}, \mathrm{pH}$ 7.5) and kept on ice. All test compounds were dissolved in 100\% DMSO and were diluted to a final 
concentration of $1 \%$ DMSO in each reaction. All readings were taken on a Varian Cary Eclipse fluorometer at room temperature. Excitation and emission wavelengths were both set to $320 \mathrm{~nm}$ and both excitation and emission slit widths were set to $2.5 \mathrm{~nm}$. Data points were recorded every $30 \mathrm{sec}$.

In a $400 \mathrm{uL} \mathrm{Hellma}{ }^{\circledR}$ Fluorescence Microcuvette (Sigma-Aldrich, Z802778), 388.8 $\mu \mathrm{L}$ of Refolding buffer ( $\mathrm{pH} 7.5$, degassed) was pipetted into the cuvette followed by $4 \mu \mathrm{L}$ of Hsp27 or buffer, and $4 \mu \mathrm{L}$ of DMSO or test compound. The solution was mixed by pipetting solution up and down. The light scattering is monitored for 5 min to observe any changes due to the Hsp27 and compounds. Once completed, CS is diluted 1:125 into the reaction mixture, stirred before monitoring light scattering for $60 \mathrm{~min}$.

\section{א-casein amyloid assay}

A $200 \mu \mathrm{M}$ stock of K-casein from bovine milk (Sigma-Aldrich, C0406) was made in cold $50 \mathrm{mM}$ sodium phosphate buffer ( $\mathrm{pH}$ 7.0). The mixture was centrifuged at the maximum speed for 10 mins at $4^{\circ} \mathrm{C}$. The concentration $(\mathrm{mg} / \mathrm{ml})$ was determined by nanodrop at $280 \mathrm{~nm}$ from which the molar concentration was determined. The stock was then diluted to $100 \mu \mathrm{M}$, aliquoted and stored at $-80^{\circ} \mathrm{C}$. For each experiment, a single aliquot was thawed and kept on ice.

Using $50 \mathrm{mM}$ sodium phosphate buffer (pH 7.0), $200 \mu \mathrm{M}$ ThT, $200 \mathrm{mM}$ DTT and $60 \mu \mathrm{M}$ Hsp27 stocks were made separately and kept on ice. All test compounds were dissolved in 100\% DMSO and diluted to $10 \%$ DMSO stocks with the buffer.

All readings were taken on either a SpectraMax M3 or FlexStation 3 multimode plate reader at $37^{\circ} \mathrm{C}$. Excitation and emission wavelengths were 440 and $480 \mathrm{~nm}$ respectively. Data points were recorded every 5 mins for 250 cycles using 20 flashes/well and a low PMT.

In a 96-well black clear-bottom plate (Greiner, 655900) on ice, $27 \mu \mathrm{L}$ of cold phosphate buffer was added to each well followed by the $6 \mu \mathrm{L}$ of test peptide or $10 \%$ DMSO in buffer, $15 \mu \mathrm{L}$ of $\kappa$-casein, $6 \mu \mathrm{L}$ of ThT and $6 \mu \mathrm{L}$ of DTT. The plate was then centrifuged, shaken for $10 \mathrm{sec}$ in the plate reader and scanned. 
When testing Hsp27, $10 \mu \mathrm{L}$ or $25 \mu \mathrm{L}$ of Hsp27 was added to the well followed by $6 \mu \mathrm{L}$ of $10 \%$ DMSO. $17 \mu \mathrm{L}$ or $2 \mu \mathrm{L}$ of cold buffer was added when testing $10 \mu \mathrm{M}$ or $25 \mu \mathrm{M}$ Hsp27 respectively. This was followed by $15 \mu \mathrm{L} \kappa$-casein, $6 \mu \mathrm{L}$ of ThT and $6 \mu \mathrm{L}$ of DTT. The plate was then centrifuged, shaken for $10 \mathrm{sec}$ in the plate reader and scanned.

\section{Experimental Procedures for Compounds}

\section{Experimental Procedures for Peptide A}

\section{Resin-O-Trp(Boc)-NH}

The resin bound amino acid Resin-O-Trp(Boc)- $\mathrm{NH}_{2}$ was synthesized following the Resin Loading procedure. A sample of 2-ClTrt $\mathrm{Cl}(1.0 \mathrm{~g}, 1.1 \mathrm{mmol} / \mathrm{g}$ loading, 1 equiv.) was placed in a solid phase tube, swelled with $\mathrm{CH}_{2} \mathrm{Cl}_{2}$ for 30 mins and then drained. $1.74 \mathrm{~g}$ Fmoc-Trp(Boc)-OH (3.3 mmol, 3 equiv.) that was pre-dissolved in the minimum volume of DIPEA in $\mathrm{CH}_{2} \mathrm{Cl}_{2}(0.40 \mathrm{M})$ was added to the swelled resin. The reaction was agitated at room temperature for 4 hours. The reaction mixture was drained, and the resin was washed according to the Resin Loading protocol to produce Resin-OTrp(Boc)-NHFmoc. A sample of resin was removed, and the resin loading was determined to be 0.633 $\mathrm{mmol} / \mathrm{g}$. The Fmoc group was then removed using the Fmoc Removal procedure to produce Resin-O$\operatorname{Trp}(\mathrm{Boc})-\mathrm{NH}_{2}$.

\section{Resin-O-Trp(Boc)-Asp(tBu)-NH2}

Resin-O-Trp(Boc)-Asp(tBu)- $\mathrm{NH}_{2}$ was synthesized according to the Coupling Reaction procedure, using Resin-O-Trp(Boc)-NH2 $(0.63$ mmol, 1 equiv.), 0.52 g Fmoc-Asp(tBu)-OH (1.27 mmol, 2 equiv.), $0.17 \mathrm{~g}$ HOBt (1.27 mmol, 2 equiv.), $0.40 \mathrm{~mL}$ DIC (2.5 mmol, 4 equiv.) and $3.82 \mathrm{~mL}$ of DMF to generate a concentration of $0.3 \mathrm{M}$. The coupling reaction was run for 4 hours and a negative ninhydrin test was used to confirm reaction completion. The reaction mixture was drained to afford Resin-O-Trp(Boc)$\mathrm{Asp}(\mathrm{tBu})-\mathrm{NHFmoc}$. The Fmoc group was then removed following the Fmoc Removal procedure to afford Resin-O-Trp(Boc)-Asp(tBu)-NH . 


\section{Resin-O-Trp(Boc)-Asp(tBu)-Arg(Pbf)-NH2}

Resin-O-Trp(Boc)-Asp(tBu)-Arg(Pbf)- $\mathrm{NH}_{2}$ was synthesized according to the Coupling Reaction procedure, using Resin-O-Trp(Boc)-Asp(tBu)- $\mathrm{NH}_{2}$ from the previous reaction, $0.82 \mathrm{~g}$ Fmoc-Arg( $\left.\mathrm{Pbf}\right)-$ OH (1.27 mmol, 2 equiv), $2.1 \mathrm{~mL}$ HOAt (1.27 mmol, 2 equiv.), $0.40 \mathrm{~mL}$ DIC (2.5 mmol, 4 equiv.) and $1.72 \mathrm{~mL}$ of DMF to generate a concentration of $0.3 \mathrm{M}$. The coupling reaction was run overnight and the reaction mixture was drained. The resin was washed with DMF ( $3 \times 1 \mathrm{~min})$ before repeating the coupling reaction under the same conditions for 4 hours. A negative ninhydrin test was used to confirm reaction completion. The reaction mixture was drained to afford Resin-O-Trp(Boc)-Asp(tBu)-Arg(Pbf)NHFmoc. The Fmoc group was then removed following the Fmoc Removal procedure to afford ResinO-Trp(Boc)-Asp(tBu)-Arg(Pbf)- $\mathrm{NH}_{2}$.

\section{Resin-O-Trp(Boc)-Asp(tBu)-Arg(Pbf)-Phe-NH}

Resin-O-Trp(Boc)-Asp(tBu)-Arg(Pbf)-Phe- $\mathrm{NH}_{2}$ was synthesized according to the Coupling Reaction procedure, using Resin-O-Trp(Boc)-Asp(tBu)-Arg $(\mathrm{Pbf})-\mathrm{NH}_{2}$ from the previous reaction, $0.49 \mathrm{~g}$ FmocPhe-OH (1.27 mmol, 2 equiv), $2.1 \mathrm{~mL}$ HOAt (1.27 mmol, 2 equiv.), $0.40 \mathrm{~mL}$ DIC (2.5 mmol, 4 equiv.) and $1.72 \mathrm{~mL}$ of DMF to generate a concentration of $0.3 \mathrm{M}$. The coupling reaction was run for 4 hours and a negative ninhydrin test was used to confirm reaction completion. The reaction mixture was drained to afford Resin-O-Trp(Boc)-Asp(tBu)-Arg(Pbf)-Phe-NHFmoc. The Fmoc group was then removed following the Fmoc Removal procedure to afford Resin-O-Trp(Boc)-Asp(tBu)-Arg(Pbf)-Phe$\mathrm{NH}_{2}$.

\section{Resin-O-Trp(Boc)-Asp(tBu)-Arg(Pbf)-Phe-Pro-NH}

Resin-O-Trp(Boc)-Asp(tBu)-Arg(Pbf)-Phe-Pro-NH2 was synthesized according to the Coupling Reaction procedure, using Resin-O-Trp(Boc)-Asp(tBu)-Arg( $\mathrm{Pbf})-\mathrm{Phe}-\mathrm{NH}_{2}$ from the previous reaction, 0.43 g Fmoc-Pro-OH (1.27 mmol, 2 equiv), 2.1 mL HOAt (1.27 mmol, 2 equiv.), 0.40 mL DIC (2.5 mmol, 4 equiv.) and $1.72 \mathrm{~mL}$ of DMF to generate a concentration of $0.3 \mathrm{M}$. The coupling reaction was 
run overnight and a negative ninhydrin test was used to confirm reaction completion. The reaction mixture was drained to afford Resin-O-Trp(Boc)-Asp(tBu)-Arg(Pbf)-Phe-Pro-NFmoc was then removed following the Fmoc Removal procedure to afford Resin-O-Trp(Boc)-Asp(tBu)-Arg(Pbf)-PhePro-NH.

\section{Resin-O-Trp(Boc)-Asp(tBu)-Arg(Pbf)-Phe-Pro-Asp(tBu)-NH 2}

Resin-O-Trp(Boc)-Asp(tBu)-Arg(Pbf)-Phe-Pro-Asp(tBu)- $\mathrm{NH}_{2}$ was synthesized according to the Coupling Reaction procedure, using Resin-O-Trp(Boc)-Asp(tBu)-Arg(Pbf)-Phe-Pro-NH from the previous reaction, $0.52 \mathrm{~g}$ Fmoc-Asp(tBu)-OH (1.27 mmol, 2 equiv), $2.1 \mathrm{~mL}$ HOAt (1.27 mmol, 2 equiv.), $0.40 \mathrm{~mL}$ DIC (2.5 mmol, 4 equiv.) and $1.72 \mathrm{~mL}$ of DMF to generate a concentration of $0.3 \mathrm{M}$. The coupling reaction was run for 8 hours and the reaction mixture was drained. A small portion of resin-bound peptide was cleaved with TFE: $\mathrm{CH}_{2} \mathrm{Cl}_{2}(1: 1 \mathrm{v} / \mathrm{v})$, filtered and the filtrate was dried under a stream of nitrogen. The sample was redissolved in acetonitrile/milli-Q water $(1: 1 \mathrm{v} / \mathrm{v})$ at a concentration of $1 \mathrm{mg} / \mathrm{ml}$ and was further diluted to $50 \mathrm{ppm}$. The sample was analysed by LC/MS where the reaction was determined to be completed by a change in the $\mathrm{m} / \mathrm{z}$ of the major product. The Fmoc group on Resin-O-Trp(Boc)-Asp(tBu)-Arg(Pbf)-Phe-Pro-Asp(tBu)-NHFmoc was then removed following the Fmoc Removal procedure to afford Resin-O-Trp(Boc)-Asp(tBu)-Arg(Pbf)-Phe-Pro-Asp(tBu)-NH2.

\section{Resin-O-Trp(Boc)-Asp(tBu)-Arg(Pbf)-Phe-Pro-Asp(tBu)-Trp(Boc)-NH2}

Resin-O-Trp(Boc)-Asp(tBu)-Arg(Pbf)-Phe-Pro-Asp(tBu)-Trp(Boc)- $\mathrm{NH}_{2}$ was synthesized according to the Coupling Reaction procedure, using Resin-O-Trp(Boc)-Asp(tBu)-Arg(Pbf)-Phe-Pro-Asp(tBu)- $\mathrm{NH}_{2}$ from the previous reaction, $0.67 \mathrm{~g}$ Fmoc-Trp(Boc)-OH (1.27 mmol, 2 equiv), $0.17 \mathrm{~g} \mathrm{HOBt}$ (1.27 mmol, 2 equiv.), $0.40 \mathrm{~mL}$ DIC (2.5 mmol, 4 equiv.) and $3.82 \mathrm{~mL}$ of DMF to generate a concentration of $0.3 \mathrm{M}$. The coupling reaction was run overnight and a negative ninhydrin test was used to confirm reaction completion. The reaction mixture was drained to afford Resin-O-Trp(Boc)-Asp(tBu)-Arg(Pbf)-Phe-Pro- 
Asp(tBu)-Trp(Boc)-NHFmoc was then removed following the Fmoc Removal procedure to afford Resin-O-Trp(Boc)-Asp(tBu)-Arg(Pbf)-Phe-Pro-Asp(tBu)-Trp(Boc)- $\mathrm{NH}_{2}$.

\section{Resin-O-Trp(Boc)-Asp(tBu)-Arg(Pbf)-Phe-Pro-Asp(tBu)-Trp(Boc)-Ser(tBu)-NH}

Resin-O-Trp(Boc)-Asp(tBu)-Arg(Pbf)-Phe-Pro-Asp(tBu)-Trp(Boc)-Ser(tBu)- $\mathrm{NH}_{2}$ was synthesized according to the Coupling Reaction procedure, using Resin-O-Trp(Boc)-Asp(tBu)-Arg(Pbf)-Phe-Pro$\operatorname{Asp}(\mathrm{tBu})-\operatorname{Trp}(\mathrm{Boc})-\mathrm{NH}_{2}$ from the previous reaction, $0.49 \mathrm{~g}$ Fmoc-Ser(tBu)-OH (1.27 mmol, 2 equiv), 2.1 mL HOAt (1.27 mmol, 2 equiv.), $0.40 \mathrm{~mL}$ DIC (2.5 mmol, 4 equiv.) and $1.72 \mathrm{~mL}$ of DMF to generate a concentration of $0.3 \mathrm{M}$. The coupling reaction was run for 8 hours and a negative ninhydrin test was used to confirm reaction completion. The reaction mixture was drained to afford Resin-O$\operatorname{Trp}(\mathrm{Boc})-\mathrm{Asp}(\mathrm{tBu})-\mathrm{Arg}(\mathrm{Pbf})-\mathrm{Phe}-\mathrm{Pro}-\mathrm{Asp}(\mathrm{tBu})-\mathrm{Trp}(\mathrm{Boc})-\mathrm{Ser}(\mathrm{tBu})-\mathrm{NHFmoc}$ was then removed following the Fmoc Removal procedure to afford Resin-O-Trp(Boc)-Asp(tBu)-Arg(Pbf)-Phe-Pro$\operatorname{Asp}(\mathrm{tBu})-\operatorname{Trp}(\mathrm{Boc})-\mathrm{Ser}(\mathrm{tBu})-\mathrm{NH}_{2}$.

\section{Resin-O-Trp(Boc)-Asp(tBu)-Arg(Pbf)-Phe-Pro-Asp(tBu)-Trp(Boc)-Ser(tBu)-Pro-NH}

Resin-O-Trp(Boc)-Asp(tBu)-Arg(Pbf)-Phe-Pro-Asp(tBu)-Trp(Boc)-Ser(tBu)-Pro-NH2 was synthesized according to the Coupling Reaction procedure, using Resin-O-Trp(Boc)-Asp(tBu)-Arg(Pbf)-Phe-Pro$\operatorname{Asp}(\mathrm{tBu})-\operatorname{Trp}(\mathrm{Boc})-\mathrm{Ser}(\mathrm{tBu})-\mathrm{NH}_{2}$ from the previous reaction, $0.43 \mathrm{~g}$ Fmoc-Pro-OH (1.27 mmol, 2 equiv), $2.1 \mathrm{~mL}$ HOAt (1.27 mmol, 2 equiv.), $0.40 \mathrm{~mL}$ DIC (2.5 mmol, 4 equiv.) and $1.72 \mathrm{~mL}$ of DMF to generate a concentration of $0.3 \mathrm{M}$. The coupling reaction was run overnight and a negative ninhydrin test was used to confirm reaction completion. The reaction mixture was drained to afford Resin-O$\operatorname{Trp}(\mathrm{Boc})-\mathrm{Asp}(\mathrm{tBu})-\mathrm{Arg}(\mathrm{Pbf})-\mathrm{Phe}-\mathrm{Pro}-\mathrm{Asp}(\mathrm{tBu})-\operatorname{Trp}(\mathrm{Boc})-\mathrm{Ser}(\mathrm{tBu})-\mathrm{Pro}-\mathrm{NFmoc}$ was then removed following the Fmoc Removal procedure to afford Resin-O-Trp(Boc)-Asp(tBu)-Arg(Pbf)-Phe-Pro$\operatorname{Asp}(\mathrm{tBu})-\operatorname{Trp}(\mathrm{Boc})-\mathrm{Ser}(\mathrm{tBu})-\mathrm{Pro}-\mathrm{NH}$. 


\section{Resin-O-Trp(Boc)-Asp(tBu)-Arg(Pbf)-Phe-Pro-Asp(tBu)-Trp(Boc)-Ser(tBu)-Pro-Gly-NH2}

Resin-O-Trp(Boc)-Asp(tBu)-Arg(Pbf)-Phe-Pro-Asp(tBu)-Trp(Boc)-Ser(tBu)-Pro-Gly-NH $\mathrm{N}_{2}$ was synthesized according to the Coupling Reaction procedure, using Resin-O-Trp(Boc)-Asp(tBu)$\operatorname{Arg}(\mathrm{Pbf})-\mathrm{Phe}-\mathrm{Pro}-\mathrm{Asp}(\mathrm{tBu})-\operatorname{Trp}(\mathrm{Boc})-\mathrm{Ser}(\mathrm{tBu})-\mathrm{Pro}-\mathrm{NH}$ from the previous reaction, $0.33 \mathrm{~g}$ Fmoc-Gly$\mathrm{OH}$ (1.27 mmol, 2 equiv), $2.1 \mathrm{~mL}$ HOAt (1.27 mmol, 2 equiv.), $0.40 \mathrm{~mL}$ DIC (2.5 mmol, 4 equiv.) and $1.72 \mathrm{~mL}$ of DMF to generate a concentration of $0.3 \mathrm{M}$. The coupling reaction was run overnight and a negative ninhydrin test was used to confirm reaction completion. The reaction mixture was drained and a small portion of resin-bound peptide was cleaved with TFE: $\mathrm{CH}_{2} \mathrm{Cl}_{2}(1: 1 \mathrm{v} / \mathrm{v})$, filtered and the filtrate was dried under a stream of nitrogen. The sample was redissolved in acetonitrile/milli-Q water $(1: 1 \mathrm{v} / \mathrm{v})$ at a concentration of $1 \mathrm{mg} / \mathrm{ml}$ and was further diluted to $50 \mathrm{ppm}$. The sample was analysed by LC/MS where the reaction was determined to be completed by a change in the $\mathrm{m} / \mathrm{z}$ of the major product. The Fmoc group on the Resin-O-Trp(Boc)-Asp(tBu)-Arg(Pbf)-Phe-Pro-Asp(tBu)-Trp(Boc)-Ser(tBu)-ProGly-NHFmoc was then removed following the Fmoc Removal procedure to afford Resin-O-Trp(Boc)$\operatorname{Asp}(\mathrm{tBu})-\operatorname{Arg}(\mathrm{Pbf})-\mathrm{Phe}-\mathrm{Pro}-\mathrm{Asp}(\mathrm{tBu})-\mathrm{Trp}(\mathrm{Boc})-\mathrm{Ser}(\mathrm{tBu})-\mathrm{Pro}-\mathrm{Gly}-\mathrm{NH}_{2}$.

\section{HO-Trp(Boc)-Asp(tBu)-Arg(Pbf)-Phe-Pro-Asp(tBu)-Trp(Boc)-Ser(tBu)-Pro-Gly-NH2}

The protected linear peptide HO-Trp(Boc)-Asp(tBu)-Arg(Pbf)-Phe-Pro-Asp(tBu)-Trp(Boc)-Ser(tBu)Pro-Gly- $\mathrm{NH}_{2}$ was generated following the Resin Cleavage procedure. The linear peptide was cleaved from $0.80 \mathrm{~g}$ of the resin using a solution of $4.0 \mathrm{~mL}$ of trifluoroethanol and $4.0 \mathrm{~mL}$ of $\mathrm{CH}_{2} \mathrm{Cl}_{2}$. $\mathrm{The}$ resin containing solution was filtered and dried in vacuo to yield the protected linear pentapeptide as a paleyellow solid (355 mg, 37\% overall).

\section{HO-Trp-Asp-Arg-Phe-Pro-Asp-Trp-Ser-Pro-Gly-NH2 (Peptide A)}

The deprotected linear peptide was generated following the Global Deprotection procedure using $0.30 \mathrm{~g}$ HO-Trp(Boc)-Asp(tBu)-Arg(Pbf)-Phe-Pro-Asp(tBu)-Trp(Boc)-Ser(tBu)-Pro-Gly-NH2 (0.16 mmol, 1 equiv.), $1.2 \mathrm{~mL}$ of a mixed solution of TFA and $\mathrm{CH}_{2} \mathrm{Cl}_{2}(9: 1 \mathrm{v} / \mathrm{v})$ and $0.21 \mathrm{~mL}$ anisole $(1.91 \mathrm{mmol}, 12$ 
equiv.) to remove the side chain protecting groups. The reaction mixture was stirred overnight and monitored via LC/MS. Upon completion, the reaction mixture was worked up as described in the Global Deprotection procedure to afford the crude linear peptide. The crude product was purified using HPLC, then lyophilised to generate pure compound as a white solid ( $25 \mathrm{mg}, 13 \%$ overall). The purity of the final compound was found to be $>95 \%$ by ${ }^{1} \mathrm{H}$ NMR, 2-D NMR (COSY, HSQC, HMBC) and LC/MS.

LC/MS (ESI) $m / z:[\mathrm{M}+\mathrm{H}]^{+}$calculated for $\mathrm{C}_{60} \mathrm{H}_{75} \mathrm{~N}_{15} \mathrm{O}_{16}{ }^{+}, 1262.55$; found 1263.40 .

${ }^{1} \mathrm{H}$ NMR (600 MHz, D $2 \mathrm{O}$ ): $\delta$ 7.61-7.49 (m, 4H, ArH Trp), 7.44-7.36 (m, 4H, ArH Phe), 7.28-7.01 (m, 7H, ArH Trp), 4.72-4.67 (m, 1H, $\alpha \mathrm{CH}$ Asp), 4.64-4.55 (m, 3H, $\alpha \mathrm{CH}$ Asp, $\alpha \mathrm{CH}$ Ser, $\alpha \mathrm{CH}$ Phe), 4.434.35 (m, 2H, $\alpha \mathrm{CH}$ Trp, $\alpha \mathrm{CH}$ Pro), 4.31 (t, $J=5.26 \mathrm{~Hz}, 1 \mathrm{H}, \alpha \mathrm{CH}$ Trp), 4.02-3.95 (m, 2H, $\alpha \mathrm{CH}$ Arg, $\alpha \mathrm{CH}$ Pro), 3.87 (s, $2 \mathrm{H}, \alpha \mathrm{CH}_{2}$ Gly) 3.81-3.75 (m, $2 \mathrm{H}, \beta \mathrm{CH}_{2} \mathrm{Ser}$ ), 3.49-3.36 (m, $2 \mathrm{H}, \delta \mathrm{CH}_{2}$ Pro), 3.49-3.36 \& 3.11-3.03 (m, $2 \mathrm{H}, \delta \mathrm{CH}_{2}$ Pro), 3.30-3.23 \& 3.23-3.12 (m, $\left.2 \mathrm{H}, \beta \mathrm{CH}_{2} \mathrm{Trp}\right), 3.23-3.12\left(\mathrm{~m}, 2 \mathrm{H}, \beta \mathrm{CH}_{2}\right.$ Trp), 3.12-3.03 \& 2.90-2.82 (m, 2H, $\left.\beta \mathrm{CH}_{2} \mathrm{Phe}\right), 2.81-2.75$ (m, 2H, $\left.\delta \mathrm{CH}_{2} \mathrm{Arg}\right), 2.81-2.75 \& 2.71-2.57$ ((m, 2H, $\left.\beta \mathrm{CH}_{2} \mathrm{Asp}\right), 2.71-2.57 \& 2.46-2.40\left(\mathrm{~m}, 2 \mathrm{H}, \beta \mathrm{CH}_{2} \mathrm{Asp}\right), 2.11-2.03 \& 1.65-1.54\left(\mathrm{~m}, 2 \mathrm{H}, \beta \mathrm{CH}_{2}\right.$ Pro), 1.94-1.72 (m, 2H, $\beta \mathrm{CH}_{2}$ Pro), 1.94-1.72 (m, $2 \mathrm{H}, \gamma \mathrm{CH}_{2}$ Pro), 1.66-1.54 \& 1.49-1.32 (m, $2 \mathrm{H}, \gamma \mathrm{CH}_{2}$ Pro), 1.49-1.32 (m, 2H, $\left.\beta \mathrm{CH}_{2} \mathrm{Arg}\right), 1.22-1.12$ (m, 2H, $\left.\gamma \mathrm{CH}_{2} \mathrm{Arg}\right)$.

\section{Experimental Procedures for Peptide B}

\section{Resin-O-Val-NH2}

The resin bound amino acid Resin-O-Val- $\mathrm{NH}_{2}$ was synthesized following the Resin Loading procedure. A sample of 2-ClTrt $\mathrm{Cl}$ resin $(1.0 \mathrm{~g}, 1.1 \mathrm{mmol} / \mathrm{g}$ loading, 1 equiv.) was placed in a solid phase tube, swelled with $\mathrm{CH}_{2} \mathrm{Cl}_{2}$ for 30 mins and then drained. $1.12 \mathrm{~g}$ Fmoc-Val-OH (3.3 mmol, 3 equiv.) that was pre-dissolved in the minimum volume of DIPEA in $\mathrm{CH}_{2} \mathrm{Cl}_{2}(0.40 \mathrm{M})$ was added to the swelled resin. The reaction was agitated at room temperature for 4 hours. The reaction mixture was drained, and the resin was washed according to the Resin Loading protocol to produce Resin-O-Trp(Boc)-NHFmoc. A 
sample of resin was removed, and the resin loading was determined to be $0.764 \mathrm{mmol} / \mathrm{g}$. The Fmoc group was then removed using the Fmoc Removal procedure to produce Resin-O-Trp(Boc)- $\mathrm{NH}_{2}$.

\section{Resin-O-Val-Gly-NH2}

Resin-O-Val-Gly- $\mathrm{NH}_{2}$ was synthesized according to the Coupling Reaction procedure, using Resin-OVal-NH 2 (0.762 mmol, 1 equiv.), 0.45 g Fmoc-Asp(tBu)-OH (1.53 mmol, 2 equiv.), 0.21 g HOBt (1.53 mmol, 2 equiv.), $0.49 \mathrm{~mL}$ DIC (3.1 mmol, 4 equiv.) and $2.06 \mathrm{~mL}$ of DMF to generate a concentration of 0.3 M. The coupling reaction was run overnight and a negative ninhydrin test was used to confirm reaction completion. The reaction mixture was drained to afford Resin-O-Val-Gly-NHFmoc. The Fmoc group was then removed following the Fmoc Removal procedure to afford Resin-O-Val-Gly-NH2.

\section{Resin-O-Val-Gly-Ser(tBu)-NH2}

Resin-O-Val-Gly-Ser(tBu)-NH 2 was synthesized according to the Coupling Reaction procedure, using Resin-O-Val-Gly- $\mathrm{NH}_{2}$ from the previous reaction, $0.59 \mathrm{~g}$ Fmoc-Ser(tBu)-OH (1.53 mmol, 2 equiv.), $0.21 \mathrm{~g} \mathrm{HOBt}$ (1.53 mmol, 2 equiv.), $0.49 \mathrm{~mL}$ DIC (3.1 mmol, 4 equiv.) and $2.06 \mathrm{~mL}$ of DMF to generate a concentration of $0.3 \mathrm{M}$. The coupling reaction was run for 4 hours and a negative ninhydrin test was used to confirm reaction completion. The reaction mixture was drained to afford Resin-O-ValGly-Ser(tBu)-NHFmoc. The Fmoc group was then removed following the Fmoc Removal procedure to afford Resin-O-Val-Gly-Ser(tBu)- $\mathrm{NH}_{2}$.

\section{Resin-O-Val-Gly-Ser(tBu)-Ser(tBu)-NH2}

Resin-O-Val-Gly-Ser(tBu)-Ser(tBu)- $\mathrm{NH}_{2}$ was synthesized according to the Coupling Reaction procedure, using Resin-O-Val-Gly-Ser(tBu)- $\mathrm{NH}_{2}$ from the previous reaction, $0.59 \mathrm{~g}$ Fmoc-Ser(tBu)-OH (1.53 mmol, 2 equiv.), $0.21 \mathrm{~g} \mathrm{HOBt} \mathrm{(1.53} \mathrm{mmol,} 2$ equiv.), $0.49 \mathrm{~mL}$ DIC (3.1 mmol, 4 equiv.) and 2.06 $\mathrm{mL}$ of DMF to generate a concentration of $0.3 \mathrm{M}$. The coupling reaction was run for 4 hours and a negative ninhydrin test was used to confirm reaction completion. The reaction mixture was drained to 
afford Resin-O-Val-Gly-Ser(tBu)-Ser(tBu)-NHFmoc. The Fmoc group was then removed following the Fmoc Removal procedure to afford Resin-O-Val-Gly-Ser(tBu)-Ser(tBu)- $\mathrm{NH}_{2}$.

\section{Resin-O-Val-Gly-Ser(tBu)-Ser(tBu)-Leu-NH}

Resin-O-Val-Gly-Ser(tBu)-Ser(tBu)-Leu- $\mathrm{NH}_{2}$ was synthesized according to the Coupling Reaction procedure, using Resin-O-Val-Gly-Ser( $(\mathrm{Bu})-\mathrm{NH}_{2}$ from the previous reaction, $0.54 \mathrm{~g}$ Fmoc-Leu-OH (1.53 mmol, 2 equiv.), $0.21 \mathrm{~g} \mathrm{HOBt}$ (1.53 mmol, 2 equiv.), $0.49 \mathrm{~mL}$ DIC (3.1 mmol, 4 equiv.) and 4.6 $\mathrm{mL}$ of DMF to generate a concentration of $0.3 \mathrm{M}$. The coupling reaction was run overnight and a negative ninhydrin test was used to confirm reaction completion. The reaction mixture was drained to afford Resin-O-Val-Gly-Ser(tBu)-Ser(tBu)-Leu-NHFmoc. The Fmoc group was then removed following the Fmoc Removal procedure to afford Resin-O-Val-Gly-Ser(tBu)-Ser(tBu)-Leu-NH 2 .

\section{Resin-O-Val-Gly-Ser(tBu)-Ser(tBu)-Leu-GIn(Trt)-NH2}

Resin-O-Val-Gly-Ser(tBu)-Ser(tBu)-Leu-Gln(Trt)- $\mathrm{NH}_{2}$ was synthesized according to the Coupling Reaction procedure, using Resin-O-Val-Gly-Ser(tBu)-Ser( $\mathrm{tBu})-\mathrm{Leu}-\mathrm{NH}_{2}$ from the previous reaction, 0.93 g Fmoc-Gln(Trt)-OH (1.53 mmol, 2 equiv.), 2.55 mL HOAt (1.53 mmol, 2 equiv.), 0.49 mL DIC (3.1 mmol, 4 equiv.) and $2.06 \mathrm{~mL}$ of DMF to generate a concentration of $0.3 \mathrm{M}$. The coupling reaction was run for 4 hours and a negative ninhydrin test was used to confirm reaction completion. The reaction mixture was drained to afford Resin-O-Val-Gly-Ser(tBu)-Ser(tBu)-Leu-NHFmoc. The Fmoc group was then removed following the Fmoc Removal procedure to afford Resin-O-Val-Gly-Ser(tBu)-Ser(tBu)Leu-Gln(Trt)-NH2.

\section{Resin-O-Val-Gly-Ser(tBu)-Ser(tBu)-Leu-GIn(Trt)-Arg(Pbf)-NH2}

Resin-O-Val-Gly-Ser(tBu)-Ser(tBu)-Leu-Gln(Trt)-Arg(Pbf)-NH 2 was synthesized according to the Coupling Reaction procedure, using Resin-O-Val-Gly-Ser(tBu)-Ser(tBu)-Leu-Gln(Trt)- $\mathrm{NH}_{2}$ from the previous reaction, $0.99 \mathrm{~g}$ Fmoc-Arg(Pbf)-OH (1.53 mmol, 2 equiv.), $2.55 \mathrm{~mL}$ HOAt (1.53 mmol, 2 
equiv.), $0.49 \mathrm{~mL}$ DIC (3.1 mmol, 4 equiv.) and $2.06 \mathrm{~mL}$ of DMF to generate a concentration of $0.3 \mathrm{M}$. The coupling reaction was run overnight and the reaction mixture was drained. The resin was washed with DMF (3 x $1 \mathrm{~min})$ before repeating the coupling reaction under the same conditions for 6 hours. A negative ninhydrin test was used to confirm reaction completion. The reaction mixture was drained to afford Resin-O-Val-Gly-Ser(tBu)-Ser(tBu)-Leu-Arg(Pbf)-NHFmoc. The Fmoc group was then removed following the Fmoc Removal procedure to afford Resin-O-Val-Gly-Ser(tBu)-Ser(tBu)-Leu-Gln(Trt)$\operatorname{Arg}(\mathrm{Pbf})-\mathrm{NH}_{2}$.

\section{Resin-O-Val-Gly-Ser(tBu)-Ser(tBu)-Leu-Gln(Trt)-Arg(Pbf)-Ser(tBu)-NH}

Resin-O-Val-Gly-Ser(tBu)-Ser(tBu)-Leu-Gln(Trt)-Arg(Pbf)-Ser(tBu)-NH 2 was synthesized according to the Coupling Reaction procedure, using Resin-O-Val-Gly-Ser(tBu)-Ser(tBu)-Leu-Gln(Trt)-Arg(Pbf)$\mathrm{NH}_{2}$ from the previous reaction, $0.59 \mathrm{~g}$ Fmoc-Ser(tBu)-OH (1.53 mmol, 2 equiv.), $2.55 \mathrm{~mL}$ HOAt (1.53 mmol, 2 equiv.), $0.49 \mathrm{~mL}$ DIC (3.1 mmol, 4 equiv.) and $2.06 \mathrm{~mL}$ of DMF to generate a concentration of $0.3 \mathrm{M}$. The coupling reaction was run for 4 hours and a negative ninhydrin test was used to confirm reaction completion. The reaction mixture was drained to afford Resin-O-Val-Gly-Ser(tBu)-Ser(tBu)Leu-Arg(Pbf)-Ser(tBu)-NHFmoc. The Fmoc group was then removed following the Fmoc Removal procedure to afford Resin-O-Val-Gly-Ser(tBu)-Ser(tBu)-Leu-Gln(Trt)-Arg(Pbf)-Ser(tBu)-NH2.

\section{Resin-O-Val-Gly-Ser(tBu)-Ser(tBu)-Leu-GIn(Trt)-Arg(Pbf)-Ser(tBu)-Leu-NH2}

Resin-O-Val-Gly-Ser(tBu)-Ser(tBu)-Leu-Gln(Trt)-Arg(Pbf)-Ser(tBu)-Leu-NH $\mathrm{N}_{2}$ was synthesized according to the Coupling Reaction procedure, using Resin-O-Val-Gly-Ser(tBu)-Ser(tBu)-Leu$\mathrm{Gln}(\mathrm{Trt})-\mathrm{Arg}(\mathrm{Pbf})-\mathrm{Ser}(\mathrm{tBu})-\mathrm{NH}_{2}$ from the previous reaction, $0.54 \mathrm{~g}$ Fmoc-Leu-OH (1.53 mmol, 2 equiv.), $2.55 \mathrm{~mL}$ HOAt (1.53 mmol, 2 equiv.), $0.49 \mathrm{~mL}$ DIC (3.1 mmol, 4 equiv.) and $2.06 \mathrm{~mL}$ of DMF to generate a concentration of $0.3 \mathrm{M}$. The coupling reaction was run overnight and a negative ninhydrin test was used to confirm reaction completion. The reaction mixture was drained to afford Resin-O-ValGly-Ser(tBu)-Ser(tBu)-Leu-Arg(Pbf)-Ser(tBu)-Leu-NHFmoc. The Fmoc group was then removed 
following the Fmoc Removal procedure to afford Resin-O-Val-Gly-Ser(tBu)-Ser(tBu)-Leu-Gln(Trt)$\operatorname{Arg}(\mathrm{Pbf})-\mathrm{Ser}(\mathrm{tBu})-\mathrm{Leu}-\mathrm{NH}_{2}$.

\section{Resin-O-Val-Gly-Ser(tBu)-Ser(tBu)-Leu-GIn(Trt)-Arg(Pbf)-Ser(tBu)-Leu-Ala-NH2}

Resin-O-Val-Gly-Ser(tBu)-Ser(tBu)-Leu-Gln(Trt)-Arg(Pbf)-Ser(tBu)-Leu-Ala- $\mathrm{NH}_{2}$ was synthesized according to the Coupling Reaction procedure, using Resin-O-Val-Gly-Ser(tBu)-Ser(tBu)-Leu$\mathrm{Gln}(\mathrm{Trt})-\mathrm{Arg}(\mathrm{Pbf})-\mathrm{Ser}(\mathrm{tBu})-\mathrm{Leu}-\mathrm{NH}_{2}$ from the previous reaction, $0.48 \mathrm{~g}$ Fmoc-Ala-OH (1.53 mmol, 2 equiv.), $2.55 \mathrm{~mL}$ HOAt (1.53 mmol, 2 equiv.), $0.49 \mathrm{~mL}$ DIC (3.1 mmol, 4 equiv.) and $2.06 \mathrm{~mL}$ of DMF to generate a concentration of $0.3 \mathrm{M}$. The coupling reaction was run overnight and a negative ninhydrin test was used to confirm reaction completion. The reaction mixture was drained to afford Resin-O-ValGly-Ser(tBu)-Ser(tBu)-Leu-Gln(Trt)-Arg(Pbf)-Ser(tBu)-Leu-Ala-NHFmoc. The Fmoc group was then removed following the Fmoc Removal procedure to afford Resin-O-Val-Gly-Ser(tBu)-Ser(tBu)-Leu$\mathrm{Gln}(\mathrm{Trt})-\mathrm{Arg}(\mathrm{Pbf})-\mathrm{Ser}(\mathrm{tBu})-\mathrm{Leu}-\mathrm{Ala}-\mathrm{NH}_{2}$.

\section{HO-Val-Gly-Ser(tBu)-Ser(tBu)-Leu-GIn(Trt)-Arg(Pbf)-Ser(tBu)-Leu-Ala-NH2}

The protected linear peptide HO-Val-Gly-Ser(tBu)-Ser(tBu)-Leu-Gln(Trt)-Arg(Pbf)-Ser(tBu)-Leu-Ala$\mathrm{NH}_{2}$ was generated following the Resin Cleavage procedure. The linear peptide was cleaved from the resin using a solution of $4.5 \mathrm{~mL}$ of trifluoroethanol and $4.5 \mathrm{~mL}$ of $\mathrm{CH}_{2} \mathrm{Cl}_{2}$. The resin containing solution was filtered and dried in vacuo to yield the protected linear pentapeptide as a pale-yellow solid (458 $\mathrm{mg}$, $36 \%$ overall).

\section{HO-Val-Gly-Ser-Ser-Leu-Gln-Arg-Ser-Leu-Ala-NH 2 (Peptide B)}

The deprotected linear peptide was generated following the Global Deprotection procedure using $0.30 \mathrm{~g}$ HO-Val-Gly-Ser(tBu)-Ser(tBu)-Leu-Gln(Trt)-Arg(Pbf)-Ser(tBu)-Leu-Ala-NH2 (0.18 mmol, 1 equiv.), $1.2 \mathrm{~mL}$ of a mixed solution of TFA and $\mathrm{CH}_{2} \mathrm{Cl}_{2}(9: 1 \mathrm{v} / \mathrm{v})$ and $0.19 \mathrm{~mL}$ anisole $(1.11 \mathrm{mmol}, 10$ equiv. $)$ to remove the side chain protecting groups. The reaction mixture was stirred overnight and monitored via 
LC/MS. Upon completion, the reaction mixture was worked up as described in the Global Deprotection procedure to afford the crude linear peptide. The crude product was purified using HPLC, then lyophilised to generate pure compound as a white solid $(21 \mathrm{mg}, 11 \%$ overall). The purity of the final compound was found to be $>95 \%$ by ${ }^{1} \mathrm{H}$ NMR, 2-D NMR (COSY, HSQC, HMBC) and LC/MS. LC/MS (ESI) $m / z$ : $[\mathrm{M}+\mathrm{H}]^{+}$calculated for $\mathrm{C}_{42} \mathrm{H}_{76} \mathrm{~N}_{14} \mathrm{O}_{15}{ }^{+}, 1016.56$; found 1017.25.

${ }^{1} \mathrm{H}$ NMR (600 MHz, $\left.\mathrm{D}_{2} \mathrm{O}\right): \delta$ 4.52-4.47 (m, 1H, $\alpha \mathrm{H}$ Ser), 4.45 (t, $J=5.46 \mathrm{~Hz}, 1 \mathrm{H}, \alpha \mathrm{CH}$ Ser), 4.44-4.37 (m, 1H, $\alpha \mathrm{CH}$ Ser) 4.44-4.37 (m, 2H, $\alpha \mathrm{CH}$ Leu), 4.37-4.29 (m, 1H, $\alpha \mathrm{CH}$ Arg) 4.37-4.29 (m, 1H, $\alpha \mathrm{CH}$ Gln), $4.21\left(\mathrm{~d}, J=5.70,1 \mathrm{H}, \alpha \mathrm{CH}\right.$ Val), $4.10\left(\mathrm{dd}, J=7.12,14.24 \mathrm{~Hz}, 1 \mathrm{H}, \alpha \mathrm{CH}\right.$ Ala), $4.00\left(\mathrm{~s}, 2 \mathrm{H}, \alpha \mathrm{CH}_{2}\right.$ Gly), 3.20 (dd, J = 7.12, $\left.14.24 \mathrm{~Hz}, 2 \mathrm{H}, \delta \mathrm{CH}_{2} \mathrm{Arg}\right), 2.37$ (t, $\left.J=7.60 \mathrm{~Hz}, 2 \mathrm{H}, \gamma \mathrm{CH}_{2} \mathrm{Gln}\right), 2.21-2.06$ (m, $1 \mathrm{H}, \beta \mathrm{CH}$ Val), 2.21-2.06 \& 2.04-1.95 (m, 2H, $\left.\beta \mathrm{CH}_{2} \mathrm{Gln}\right), 1.90-1.82 \& 1.80-1.72\left(\mathrm{~m}, 1 \mathrm{H}, \beta \mathrm{CH}_{2} \mathrm{Arg}\right)$, 1.72-1.57 (m, 4H, $\beta \mathrm{CH}_{2}$ Leu) 1.72-1.57(m, 2H, $\gamma \mathrm{CH}$ Leu), 1.53 (d, $\left.J=7.13 \mathrm{~Hz}, 3 \mathrm{H}, \beta \mathrm{CH}_{3} \mathrm{Ala}\right), 0.97-$ $0.84\left(\mathrm{~m}, 12 \mathrm{H}, \delta \mathrm{CH}_{3} \mathrm{Leu}\right), 0.97-0.84\left(\mathrm{~m}, 6 \mathrm{H}, \gamma \mathrm{CH}_{3} \mathrm{Val}\right)$.

\section{Experimental Procedures for Peptide C}

\section{Resin-O-Tyr(tBu)-- $\mathrm{NH}_{2}$}

The resin bound amino acid Resin-O-Tyr(tBu)- $\mathrm{NH}_{2}$ was synthesized following the Resin Loading procedure. A sample of 2-ClTrt $\mathrm{Cl}$ resin $(1.0 \mathrm{~g}, 1.1 \mathrm{mmol} / \mathrm{g}$ loading, 1 equiv. $)$ was placed in a solid phase tube, swelled with $\mathrm{CH}_{2} \mathrm{Cl}_{2}$ for 30 mins and then drained. $1.52 \mathrm{~g}$ Fmoc-Tyr(tBu)-OH (3.3 mmol, 3 equiv.) that was pre-dissolved in the minimum volume of DIPEA in $\mathrm{CH}_{2} \mathrm{Cl}_{2}(0.40 \mathrm{M})$ was added to the swelled resin. The reaction was agitated at room temperature for 4 hours. The reaction mixture was drained, and the resin was washed according to the Resin Loading protocol to produce Resin-O$\operatorname{Tyr}(\mathrm{tBu})$-NHFmoc. A sample of resin was removed, and the resin loading was determined to be 0.715 $\mathrm{mmol} / \mathrm{g}$. The Fmoc group was then removed using the Fmoc Removal procedure to produce Resin-O$\operatorname{Tyr}(\mathrm{tBu})-\mathrm{NH}_{2}$. 


\section{Resin-O-Tyr(tBu)-Lys(Boc)--NH2}

Resin-O-Tyr(tBu)-Lys(Boc)- $\mathrm{NH}_{2}$ was synthesized according to the Coupling Reaction procedure, using Resin-O-Tyr(tBu)-NH 2 (0.715 mmol, 1 equiv.), 0.67 g Fmoc-Lys(Boc)-OH (1.43 mmol, 2 equiv.), 2.38 mL HOAt (1.43 mmol, 2 equiv.), $0.45 \mathrm{~mL}$ DIC (3.1 mmol, 4 equiv.) and $1.93 \mathrm{~mL}$ of DMF to generate a concentration of $0.3 \mathrm{M}$. The coupling reaction was run overnight and a negative ninhydrin test was used to confirm reaction completion. The reaction mixture was drained to afford Resin-O-Tyr $(\mathrm{tBu})-$ Lys(Boc)-NHFmoc. The Fmoc group was then removed following the Fmoc Removal procedure to afford Resin-O-Tyr(tBu)-Lys(Boc)- $\mathrm{NH}_{2}$.

\section{Resin-O-Tyr(tBu)-Lys(Boc)-Arg(Pbf)-NH2}

Resin-O-Tyr(tBu)-Lys(Boc)-Arg(Pbf)-NH 2 was synthesized according to the Coupling Reaction procedure, using Resin-O-Tyr(tBu)-Lys(Boc)-NH 2 (0.715 mmol, 1 equiv.), 0.93 g Fmoc-Arg(Pbf)-OH (1.43 mmol, 2 equiv.), $2.38 \mathrm{~mL}$ HOAt (1.43 mmol, 2 equiv.), $0.45 \mathrm{~mL}$ DIC (3.1 mmol, 4 equiv.) and $1.93 \mathrm{~mL}$ of DMF to generate a concentration of $0.3 \mathrm{M}$. The coupling reaction was run for 4 hours and a negative ninhydrin test was used to confirm reaction completion. The reaction mixture was drained to afford Resin-O-Tyr(tBu)-Lys(Boc)-Arg(Pbf)-NHFmoc. The Fmoc group was then removed following the Fmoc Removal procedure to afford Resin-O-Tyr(tBu)-Lys(Boc)-Arg( $\mathrm{Pbf})-\mathrm{NH}_{2}$.

\section{Resin-O-Tyr(tBu)-Lys(Boc)-Arg(Pbf)-Thr(tBu)-NH2}

Resin-O-Tyr(tBu)-Lys(Boc)-Arg(Pbf)-Thr(tBu)-NH 2 was synthesized according to the Coupling Reaction procedure, using Resin-O-Tyr(tBu)-Lys(Boc)-Arg(Pbf)- $\mathrm{NH}_{2}(0.715 \mathrm{mmol}, 1$ equiv.), $0.57 \mathrm{~g}$ Fmoc-Thr(tBu)-OH (1.43 mmol, 2 equiv.), 2.38 mL HOAt (1.43 mmol, 2 equiv.), 0.45 mL DIC (3.1 mmol, 4 equiv.) and $1.93 \mathrm{~mL}$ of DMF to generate a concentration of $0.3 \mathrm{M}$. The coupling reaction was run for 4 hours and the reaction mixture was drained. The resin was washed with DMF ( $3 \mathrm{x} 1 \mathrm{~min})$ before repeating the coupling reaction under the same conditions for overnight. A negative ninhydrin test was used to confirm reaction completion. The reaction mixture was drained to afford Resin-O- 
$\operatorname{Tyr}(\mathrm{tBu})-\mathrm{Lys}(\mathrm{Boc})-\operatorname{Arg}(\mathrm{Pbf})-\mathrm{Thr}(\mathrm{tBu})-\mathrm{NHFmoc}$. The Fmoc group was then removed following the Fmoc Removal procedure to afford Resin-O-Tyr(tBu)-Lys(Boc)-Arg( $\mathrm{Pbf})-\mathrm{Thr}(\mathrm{tBu})-\mathrm{NH}_{2}$.

\section{Resin-O-Tyr(tBu)-Lys(Boc)-Arg(Pbf)-Thr(tBu)-Phe-NH}

Resin-O-Tyr(tBu)-Lys(Boc)-Arg(Pbf)-Thr(tBu)-Phe- $\mathrm{NH}_{2}$ was synthesized according to the Coupling Reaction procedure, using Resin-O-Tyr(tBu)-Lys(Boc)-Arg(Pbf)-Thr(tBu)- $\mathrm{NH}_{2}(0.715$ mmol, 1 equiv.), 0.55 g Fmoc-Phe-OH (1.43 mmol, 2 equiv.), 2.38 mL HOAt (1.43 mmol, 2 equiv.), 0.45 mL DIC (3.1 mmol, 4 equiv.) and $1.93 \mathrm{~mL}$ of DMF to generate a concentration of $0.3 \mathrm{M}$. The coupling reaction was run for overnight and a negative ninhydrin test was used to confirm reaction completion. The reaction mixture was drained to afford Resin-O-Tyr(tBu)-Lys(Boc)-Arg(Pbf)-Thr(tBu)-Phe-NHFmoc. The Fmoc group was then removed following the Fmoc Removal procedure to afford Resin-O-Tyr(tBu)-Lys(Boc)$\operatorname{Arg}(\mathrm{Pbf})-\mathrm{Thr}(\mathrm{tBu})-\mathrm{Phe}-\mathrm{NH}_{2}$.

\section{Resin-O-Tyr(tBu)-Lys(Boc)-Arg(Pbf)-Thr(tBu)-Phe-Cys(Trt)-NH}

Resin-O-Tyr(tBu)-Lys(Boc)-Arg(Pbf)-Thr(tBu)-Phe-Cys(Trt)- $\mathrm{NH}_{2}$ was synthesized according to the Coupling Reaction procedure, using Resin-O-Tyr(tBu)-Lys(Boc)-Arg(Pbf)-Thr(tBu)-NH2 $(0.715$ mmol, 1 equiv.), 0.84 g Fmoc-Cys(Trt)-OH (1.43 mmol, 2 equiv.), 2.38 mL HOAt (1.43 mmol, 2 equiv.), 0.45 mL DIC (3.1 mmol, 4 equiv.) and $1.93 \mathrm{~mL}$ of DMF to generate a concentration of $0.3 \mathrm{M}$. The coupling reaction was run for 4 hours and a negative ninhydrin test was used to confirm reaction completion. The reaction mixture was drained to afford Resin-O-Tyr(tBu)-Lys(Boc)-Arg(Pbf)-Thr(tBu)-Phe-Cys(Trt)NHFmoc. The Fmoc group was then removed following the Fmoc Removal procedure to afford Resin$\mathrm{O}-\mathrm{Tyr}(\mathrm{tBu})-\mathrm{Lys}(\mathrm{Boc})-\operatorname{Arg}(\mathrm{Pbf})-\mathrm{Thr}(\mathrm{tBu})-\mathrm{Phe}-\mathrm{Cys}(\mathrm{Trt})-\mathrm{NH}_{2}$.

\section{Resin-O-Tyr(tBu)-Lys(Boc)-Arg(Pbf)-Thr(tBu)-Phe-Cys(Trt)-Arg(Pbf)-NH2}

Resin-O-Tyr(tBu)-Lys(Boc)-Arg(Pbf)-Thr(tBu)-Phe-Cys(Trt)-Arg(Pbf)- $\mathrm{NH}_{2}$ was synthesized according to the Coupling Reaction procedure, using Resin-O-Tyr(tBu)-Lys(Boc)-Arg(Pbf)-Thr(tBu)-Cys(Trt)- 
$\mathrm{NH}_{2}$ (0.715 mmol, 1 equiv.), 0.93 g Fmoc-Arg(Pbf)-OH (1.43 mmol, 2 equiv.), $2.38 \mathrm{~mL}$ HOAt (1.43 mmol, 2 equiv.), $0.45 \mathrm{~mL}$ DIC (3.1 mmol, 4 equiv.) and $1.93 \mathrm{~mL}$ of DMF to generate a concentration of 0.3 M. The coupling reaction was run for overnight and a negative ninhydrin test was used to confirm reaction completion. The reaction mixture was drained to afford Resin-O-Tyr(tBu)-Lys(Boc)-Arg(Pbf)$\operatorname{Thr}(\mathrm{tBu})-\mathrm{Phe}-\mathrm{Cys}(\mathrm{Trt})-\mathrm{Arg}(\mathrm{Pbf})-\mathrm{NHFmoc}$. The Fmoc group was then removed following the Fmoc Removal procedure to afford Resin-O-Tyr(tBu)-Lys(Boc)-Arg( $\mathrm{Pbf})-\mathrm{Thr}(\mathrm{tBu})-\mathrm{Phe}-\mathrm{Cys}(\mathrm{Trt})-\mathrm{Arg}(\mathrm{Pbf})-$ $\mathrm{NH}_{2}$.

\section{Resin-O-Tyr(tBu)-Lys(Boc)-Arg(Pbf)-Thr(tBu)-Phe-Cys(Trt)-Arg(Pbf)-Ser(tBu)-NH2} Resin-O-Tyr(tBu)-Lys(Boc)-Arg(Pbf)-Thr(tBu)-Phe-Cys(Trt)-Arg(Pbf)-Ser(tBu)-NH $\mathrm{NH}_{2}$ was synthesized according to the Coupling Reaction procedure, using Resin-O-Tyr(tBu)-Lys(Boc)-Arg(Pbf)-Thr(tBu)Cys(Trt)-Arg(Pbf)-NH2 (0.715 mmol, 1 equiv.), 0.55 g Fmoc-Ser(tBu)-OH (1.43 mmol, 2 equiv.), 2.38 mL HOAt (1.43 mmol, 2 equiv.), $0.45 \mathrm{~mL}$ DIC (3.1 mmol, 4 equiv.) and $1.93 \mathrm{~mL}$ of DMF to generate a concentration of $0.3 \mathrm{M}$. The coupling reaction was run for overnight and a negative ninhydrin test was used to confirm reaction completion. The reaction mixture was drained to afford Resin-O-Tyr(tBu)Lys(Boc)-Arg(Pbf)-Thr(tBu)-Phe-Cys(Trt)-Arg(Pbf)-Ser(tBu)-NHFmoc. The Fmoc group was then removed following the Fmoc Removal procedure to afford Resin-O-Tyr(tBu)-Lys(Boc)-Arg(Pbf)$\operatorname{Thr}(\mathrm{tBu})-\mathrm{Phe}-\mathrm{Cys}(\mathrm{Trt})-\mathrm{Arg}(\mathrm{Pbf})-\mathrm{Ser}(\mathrm{tBu})-\mathrm{NH}_{2}$.

\section{Resin-O-Tyr(tBu)-Lys(Boc)-Arg(Pbf)-Thr(tBu)-Phe-Cys(Trt)-Arg(Pbf)-Ser(tBu)-Ile-NH 2} Resin-O-Tyr(tBu)-Lys(Boc)-Arg(Pbf)-Thr(tBu)-Phe-Cys(Trt)-Arg(Pbf)-Ser(tBu)-Ile-NH 2 was synthesized according to the Coupling Reaction procedure, using Resin-O-Tyr(tBu)-Lys(Boc)$\operatorname{Arg}(\mathrm{Pbf})-\mathrm{Thr}(\mathrm{tBu})-\mathrm{Cys}(\mathrm{Trt})-\mathrm{Arg}(\mathrm{Pbf})-\mathrm{Ser}(\mathrm{tBu})-\mathrm{NH}_{2}$ (0.715 mmol, 1 equiv.), $0.51 \mathrm{~g}$ Fmoc-Ile-OH (1.43 mmol, 2 equiv.), $2.38 \mathrm{~mL}$ HOAt (1.43 mmol, 2 equiv.), $0.45 \mathrm{~mL}$ DIC (3.1 mmol, 4 equiv.) and $1.93 \mathrm{~mL}$ of DMF to generate a concentration of $0.3 \mathrm{M}$. The coupling reaction was run for 4 hours and the reaction mixture was drained. The resin was washed with DMF ( $3 \times 1 \mathrm{~min})$ before repeating the 
coupling reaction under the same conditions for overnight. A negative ninhydrin test was used to confirm reaction completion. The reaction mixture was drained to afford Resin-O-Tyr(tBu)-Lys(Boc)$\operatorname{Arg}(\mathrm{Pbf})-\mathrm{Thr}(\mathrm{tBu})-\mathrm{Phe}-\mathrm{Cys}(\mathrm{Trt})-\mathrm{Arg}(\mathrm{Pbf})-\mathrm{Ser}(\mathrm{tBu})-\mathrm{Ile}-\mathrm{NHFmoc}$. The Fmoc group was then removed following the Fmoc Removal procedure to afford Resin-O-Tyr(tBu)-Lys(Boc)-Arg(Pbf)-Thr(tBu)-PheCys(Trt)-Arg(Pbf)-Ser(tBu)-Ile-NH .

\section{Resin-O-Tyr(tBu)-Lys(Boc)-Arg(Pbf)-Thr(tBu)-Phe-Cys(Trt)-Arg(Pbf)-Ser(tBu)-Ile-Tyr(tBu)-} $\mathrm{NH}_{2}$

Resin-O-Tyr(tBu)-Lys(Boc)-Arg(Pbf)-Thr(tBu)-Phe-Cys(Trt)-Arg(Pbf)-Ser(tBu)-Ile- $\mathrm{NH}_{2}$ was synthesized according to the Coupling Reaction procedure, using Resin-O-Tyr(tBu)-Lys(Boc)$\operatorname{Arg}(\mathrm{Pbf})-\mathrm{Thr}(\mathrm{tBu})-\mathrm{Cys}(\mathrm{Trt})-\mathrm{Arg}(\mathrm{Pbf})-\mathrm{Ile}-\mathrm{NH}_{2}(0.715$ mmol, 1 equiv.), $0.71 \mathrm{~g}$ Fmoc-Tyr(tBu)-OH (1.43 mmol, 2 equiv.), $2.38 \mathrm{~mL}$ HOAt (1.43 mmol, 2 equiv.), $0.45 \mathrm{~mL}$ DIC (3.1 mmol, 4 equiv.) and $1.93 \mathrm{~mL}$ of DMF to generate a concentration of $0.3 \mathrm{M}$. The coupling reaction was run for overnight and a negative ninhydrin test was used to confirm reaction completion. The reaction mixture was drained to afford Resin-O-Tyr(tBu)-Lys(Boc)-Arg(Pbf)-Thr(tBu)-Phe-Cys(Trt)-Arg(Pbf)-Ser(tBu)-Ile-Tyr(tBu)NHFmoc. The Fmoc group was then removed following the Fmoc Removal procedure to afford ResinO-Tyr(tBu)-Lys(Boc)-Arg(Pbf)-Thr(tBu)-Phe-Cys(Trt)-Arg(Pbf)-Ser(tBu)-Ile-Tyr(tBu)-NH2.

\section{HO-Tyr(tBu)-Lys(Boc)-Arg(Pbf)-Thr(tBu)-Phe-Cys(Trt)-Arg(Pbf)-Ser(tBu)-Ile-Tyr(tBu)-NH2} The protected linear peptide HO-Val-Gly-Ser(tBu)-Ser(tBu)-Leu-Gln(Trt)-Arg(Pbf)-Ser(tBu)-Leu-Ala$\mathrm{NH}_{2}$ was generated following the Resin Cleavage procedure. The linear peptide was cleaved from $0.44 \mathrm{~g}$ of resin ( $0.32 \mathrm{mmol}, 1$ equiv) using a solution of $2.2 \mathrm{~mL}$ of trifluoroethanol and $2.2 \mathrm{~mL}$ of $\mathrm{CH}_{2} \mathrm{Cl}_{2}$. The resin containing solution was filtered and dried in vacuo to yield the protected linear pentapeptide as a pale-yellow solid (111 mg, 15\% overall). 


\section{HO-Tyr-Lys-Arg-Thr-Phe-Cys-Arg-Ser-Ile-Tyr-NH 2 (Peptide C)}

The deprotected linear peptide was generated following the Global Deprotection procedure using $0.11 \mathrm{~g}$ HO-Tyr(tBu)-Lys(Boc)-Arg(Pbf)-Thr(tBu)-Phe-Cys(Trt)-Arg(Pbf)-Ser(tBu)-Ile-Tyr(tBu)-NH $2(0.18$ mmol, 1 equiv.), $0.44 \mathrm{~mL}$ of a mixed solution of TFA and $\mathrm{CH}_{2} \mathrm{Cl}_{2}(9: 1 \mathrm{v} / \mathrm{v})$ and $0.06 \mathrm{~mL}$ EDT $(0.74$ mmol, 16 equiv.) to remove the side chain protecting groups. The reaction mixture was stirred overnight and monitored via LC/MS. Upon completion, the reaction mixture was worked up as described in the Global Deprotection procedure to afford the crude linear peptide. The crude product was purified using HPLC, then lyophilised to generate pure compound as a white solid ( $7.1 \mathrm{mg}, 12 \%$ overall). The purity of the final compound was found to be $>95 \%$ by ${ }^{1} \mathrm{H}$ NMR, 2-D NMR (COSY, HSQC, HMBC) and LC/MS.

LC/MS (ESI) $m / z$ : $[\mathrm{M}+2 \mathrm{Na}]^{+}$calculated for $\mathrm{C}_{61} \mathrm{H}_{93} \mathrm{~N}_{17} \mathrm{O}_{15} \mathrm{~S}^{+}, 690.83$; found 693.25.

${ }^{1} \mathrm{H}$ NMR (600 MHz, D $2 \mathrm{O}$ ): $\delta$ 7.32-7.14 (m, 5H, ArH Phe), 7.08-6.98 (m, 4H, ArH Tyr), 6.81-6.69 (m, 4H, ArH Tyr), 4.68-4.56 (m, 1H, $\alpha \mathrm{CH}$ Phe), 4.68-4.56 (m, 1H, $\alpha \mathrm{CH}$ Cys), 4.60-4.49 (m, 1H, $\alpha \mathrm{CH}$ Ser), 4.60-4.49 (m, 1H, $\alpha \mathrm{CH}$ Tyr), 4.49-4.33 (m, 1H, $\alpha \mathrm{CH}$ Lys), 4.49-4.33 (m, 2H, $\alpha \mathrm{CH}$ Arg), 4.49-4.33 (m, 1H, $\alpha \mathrm{CH}$ Thr), 4.49-4.33 (m, 1H, $\alpha \mathrm{CH}$ Ile), 4.49-4.33 (m, 1H, $\alpha \mathrm{CH}$ Tyr), 4.31-4.21 (m, 1H, $\beta \mathrm{CH}$ Thr), 4.08-3.91 (m, 2H, $\beta \mathrm{CH}_{2}$ Ser), 3.47-3.40 \& 3.37-3.18 (m, $\left.2 \mathrm{H}, \beta \mathrm{CH}_{2} \mathrm{Phe}\right), 3.37-3.17$ (m, $4 \mathrm{H}, \delta \mathrm{CH}_{2} \mathrm{Arg}$ ), 3.37-3.17 (m, 4H, $\beta \mathrm{CH}_{2}$ Tyr), 3.08 (t, $\left.J=\mathrm{Hz}, 2 \mathrm{H}, \varepsilon \mathrm{CH}_{2} \mathrm{Lys}\right), 3.03-3.95$ (m, $\left.2 \mathrm{H}, \beta \mathrm{CH}_{2} \mathrm{Cys}\right), 1.94-1.37$ (m, 2H, $\beta \mathrm{CH}_{2}$ Lys), 1.94-1.37 (m, 2H, $\gamma \mathrm{CH}_{2}$ Lys), 1.94-1.37 (m, 2H, $\delta \mathrm{CH}_{2}$ Lys), 1.94-1.37 (m, 4H, $\gamma \mathrm{CH}_{2} \mathrm{Arg}$ ), 1.94-1.37 (m, 4H, $\left.\beta \mathrm{CH}_{2} \mathrm{Arg}\right), 1.31-1.27$ (m, 3H, $\left.\gamma \mathrm{CH}_{3} \mathrm{Thr}\right), 1.31-1.20$ (m, $\left.2 \mathrm{H}, \gamma \mathrm{CH}_{2} \mathrm{Ile}\right)$, 0.81-0.74 (m, 3H, $\delta \mathrm{CH}_{3}$ Ile), 0.81-0.74 (m, 3H, $\gamma \mathrm{CH}_{3}$ Ile).

\section{Experimental Procedures for Peptide D}

\section{Resin-O-Phe-Thr(tBu)-Val-Pro-Ile-Thr(tBu)-Ile-Glu(tBu)-Asn(Trt)-Ser(tBu)-NH2}

Resin-bound decapeptide Resin-O-Phe-Thr(tBu)-Val-Pro-Ile-Thr(tBu)-Ile-Glu(tBu)-Asn(Trt)-Ser(tBu)$\mathrm{NH}_{2}$ was synthesised using a Biotage Initiator + Alstra Automated Microwave Peptide Synthesiser. 0.50 g H-Phe-2-ClTrt resin was weighed and added to a $10 \mathrm{~mL}$-capacity dedicated reactor vial. All amino 
acids were pre-dissolved in DMF to produce $3 \mathrm{~mL}$ solutions with concentrations of $0.25 \mathrm{M}$. Coupling reagents HOBt and HOAt were dissolved separately in a mix of DIC and DMF (1:1 v/v) also with concentrations of $0.25 \mathrm{M}$. The system was set up according to the manufacturer's protocol.

The resin underwent sequential coupling reactions with each amino acid as described in the Coupling Reaction procedure, but the reaction time was shortened to 35 minutes and the $\mathrm{R}=$ reaction temperature was increased to $70^{\circ} \mathrm{C}$.

Fmoc removal washes were performed as described in the Fmoc Removal procedure. Once the final amino acid coupling reaction was complete, the system automatically performed the pre-cleavage washes as described in the Cleavage procedure. Once the synthesis was complete, the resin-bound peptide was removed from the system and dried in vacuo overnight.

\section{HO-Phe-Thr(tBu)-Val-Pro-Ile-Thr(tBu)-Ile-Glu(tBu)-Asn(Trt)-Ser(tBu)-NH2}

The protected linear peptide HO-Phe-Thr(tBu)-Val-Pro-Ile-Thr(tBu)-Ile-Glu(tBu)-Asn(Trt)-Ser(tBu)$\mathrm{NH}_{2}$ was generated following the Resin Cleavage procedure. The linear peptide was cleaved from $0.66 \mathrm{~g}$ of resin $\left(0.50 \mathrm{mmol}, 1\right.$ equiv) using a solution of $3.3 \mathrm{~mL}$ of trifluoroethanol and $3.3 \mathrm{~mL}$ of $\mathrm{CH}_{2} \mathrm{Cl}_{2}$. The resin containing solution was filtered and dried in vacuo to yield the protected linear pentapeptide as a pale-yellow solid (227 mg, 29\% overall).

\section{HO-Phe-Thr-Val-Pro-Ile-Thr-Ile-Glu-Asn-Ser-NH2 (Peptide D)}

The deprotected linear peptide was generated following the Global Deprotection procedure using $0.09 \mathrm{~g}$ HO-Phe-Thr(tBu)-Val-Pro-Ile-Thr(tBu)-Ile-Glu(tBu)-Asn(Trt)-Ser(tBu)-NH $\mathrm{NH}_{2}(0.06$ mmol, 1 equiv.), $0.36 \mathrm{~mL}$ of a mixed solution of TFA and $\mathrm{CH}_{2} \mathrm{Cl}_{2}(9: 1 \mathrm{v} / \mathrm{v})$ and $0.06 \mathrm{~mL}$ anisole $(0.06 \mathrm{mmol}, 10$ equiv.) to remove the side chain protecting groups. The reaction mixture was stirred overnight and monitored via $\mathrm{LC} / \mathrm{MS}$. Upon completion, the reaction mixture was worked up as described in the Global Deprotection procedure to afford the crude linear peptide. The crude product was purified using HPLC, 
then lyophilised to generate pure compound as a white solid (24.7 mg, 39\% overall). The purity of the final compound was found to be $>95 \%$ by ${ }^{1} \mathrm{H}$ NMR, 2-D NMR (COSY, HSQC, HMBC) and LC/MS. LC/MS (ESI) $m / z:[\mathrm{M}+\mathrm{H}]^{+}$calculated for $\mathrm{C}_{51} \mathrm{H}_{81} \mathrm{~N}_{11} \mathrm{O}_{17}{ }^{+}, 1120.58$; found 1120.30 .

${ }^{1} \mathrm{H}$ NMR (600 MHz, DMSO-d6): $\delta 8.29(\mathrm{~d}, J=6.51 \mathrm{~Hz}, 1 \mathrm{H}, \mathrm{NH}), 7.92(\mathrm{~d}, J=8.79 \mathrm{~Hz}, 1 \mathrm{H}, \mathrm{NH}), 7.82$ (m, 1H, NH), $7.78(\mathrm{~d}, J=7.65 \mathrm{~Hz}, 1 \mathrm{H}, \mathrm{NH}), 7.67(\mathrm{~d}, J=8.34 \mathrm{~Hz}, 1 \mathrm{H}, \mathrm{NH}), 7.61(\mathrm{~d}, J=8.34 \mathrm{~Hz}, 1 \mathrm{H}$, NH), 7.35 (s, 1H, NH), 7.26-7.15 (m, 5H, ArH Phe), 6.89 (s, 1H, NH), 4.43-4.34 (m, 1H, $\alpha \mathrm{CH}$ Asp), 4.434.34 (m, 1H, $\alpha \mathrm{CH}$ Val), 4.43-4.34 (m, 1H, $\alpha \mathrm{CH}$ Thr), 4.43-4.34 (m, 1H, Glu), 4.25-4.18 (m, 1H, $\alpha \mathrm{CH}$ Thr), 4.25-4.18 (m, 2H, $\alpha \mathrm{CH}$ Ile), 4.18-4.12 (m, 1H, $\alpha \mathrm{CH}$ Ser), 3.98-3.90 (m, 2H, $\beta \mathrm{CH}$ Thr), 3.72-3.66 \& 3.55-3.48 (m, $2 \mathrm{H}, \delta \mathrm{CH}_{2}$ Pro), 3.64-3.57 (m, $1 \mathrm{H}, \alpha \mathrm{CH}$ Phe), 3.64-3.57 \& 3.55-3.48 (m, $2 \mathrm{H}, \beta \mathrm{CH}_{2}$ Ser), 3.08-3.02 (dd, $\left.J=8.79,13.82 \mathrm{~Hz}, 1 \mathrm{H}, \beta \mathrm{CH}_{2} \mathrm{Phe}\right), 2.94-2.87$ (dd, $J=6.11,13.82 \mathrm{~Hz}, 1 \mathrm{H}, \beta \mathrm{CH}_{2} \mathrm{Phe}$ ), 2.62-2.56 \& 2.53-2.45 (m, 2H, $\beta \mathrm{CH}_{2}$ Asp), 2.22-2.09 (m, $\left.2 \mathrm{H}, \gamma \mathrm{CH}_{2} \mathrm{Glu}\right), 2.02-1.92 \&$ 1.85-1.66 (m, $2 \mathrm{H}$, $\beta \mathrm{CH}_{2}$ Pro), 2.02-1.92 (m, $1 \mathrm{H}, \beta \mathrm{CH}$ Val), 1.92-1.85 \& 1.85-1.66 (m, 2H, $\gamma \mathrm{CH}_{2}$ Pro), 1.85-1.66 (m, $2 \mathrm{H}$, $\beta \mathrm{CH}_{2}$ Glu $), 1.85-1.66$ (m, 2H, $\beta \mathrm{CH}$ Ile), $1.55-1.47 \& 1.47-1.38\left(\mathrm{~m}, 2 \mathrm{H}, \gamma \mathrm{CH}_{2} \mathrm{Ile}\right), 1.11-1.02\left(\mathrm{~m}, 2 \mathrm{H}, \gamma \mathrm{CH}_{2}\right.$ Ile), 0.98 (dd, $\left.J=3.35,9.73 \mathrm{~Hz}, 6 \mathrm{H}, \gamma \mathrm{CH}_{3} \mathrm{Thr}\right), 0.87$ (d, $J=6.63 \mathrm{~Hz}, 3 \mathrm{H}, \gamma \mathrm{CH}_{3} \mathrm{Val}$ ), 0.84-0.77 (m, 3H, $\gamma \mathrm{CH}_{3} \mathrm{Val}$ ), 0.84-0.77 (m, 6H, $\left.\gamma \mathrm{CH}_{3} \mathrm{Ile}\right), 0.84-0.77$ (m, 6H, $\left.\delta \mathrm{CH}_{3} \mathrm{Ile}\right)$.

\section{Experimental Procedures for Scrambled Peptide (SP)}

\section{Resin-O-Leu- $\mathrm{NH}_{2}$}

The resin bound amino acid Resin-O-Leu- $\mathrm{NH}_{2}$ was synthesized following the Resin Loading procedure. A sample of 2-ClTrt $\mathrm{Cl}$ resin $(0.55 \mathrm{~g}, 1.14 \mathrm{mmol} / \mathrm{g}$ loading, 1 equiv. $)$ was placed in a solid phase tube, swelled with $\mathrm{CH}_{2} \mathrm{Cl}_{2}$ for 30 mins and then drained. $0.66 \mathrm{~g}$ Fmoc-Leu-OH (1.87 mmol, 3 equiv.) that was pre-dissolved in the minimum volume of DIPEA in $\mathrm{CH}_{2} \mathrm{Cl}_{2}(0.40 \mathrm{M})$ was added to the swelled resin. The reaction was agitated at room temperature for 4 hours. The reaction mixture was drained, and the resin was washed according to the Resin Loading protocol to produce Resin-O-Leu-NHFmoc. A sample of resin was removed, and the resin loading was determined to be $0.700 \mathrm{mmol} / \mathrm{g}$. The Fmoc group was then removed using the Fmoc Removal procedure to produce Resin-O-Leu-NH $\mathrm{N}_{2}$. 


\section{Resin-O-Leu-Leu-NH2}

Resin-O-Leu-Leu-NH $\mathrm{N}_{2}$ was synthesized according to the Coupling Reaction procedure, using Resin-O-

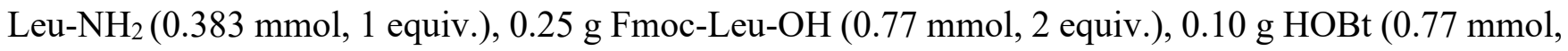
2 equiv.), $0.24 \mathrm{~mL}$ DIC (1.53 mmol, 4 equiv.) and $2.31 \mathrm{~mL}$ of DMF to generate a concentration of $0.3 \mathrm{M}$. The coupling reaction was run for 2 hours and a negative ninhydrin test was used to confirm reaction completion. The reaction mixture was drained to afford Resin-O-Leu-Leu-NHFmoc. The Fmoc group was then removed following the Fmoc Removal procedure to afford Resin-O-Leu-Leu-NH $\mathrm{NH}_{2}$

\section{Resin-O-Leu-Leu-Val-NH2}

Resin-O-Leu-Leu-Val- $\mathrm{NH}_{2}$ was synthesized according to the Coupling Reaction procedure, using ResinO-Leu-Leu-NH 2 (0.383 mmol, 1 equiv.), 0.26 g Fmoc-Val-OH (0.77 mmol, 2 equiv.), 0.10 g HOBt (0.77 mmol, 2 equiv.), $0.24 \mathrm{~mL}$ DIC (1.53 mmol, 4 equiv.) and $2.31 \mathrm{~mL}$ of DMF to generate a concentration of $0.3 \mathrm{M}$. The coupling reaction was run for 2 hours and a negative ninhydrin test was used to confirm reaction completion. The reaction mixture was drained to afford Resin-O-Leu-Leu-Val-NHFmoc. The Fmoc group was then removed following the Fmoc Removal procedure to afford Resin-O-Leu-Leu-Val$\mathrm{NH}_{2}$.

\section{Resin-O- Leu-Leu-Val-Glu(tBu)-NH2}

Resin-O-Leu-Leu-Val-Glu(tBu)- $\mathrm{NH}_{2}$ was synthesized according to the Coupling Reaction procedure, using Resin-O-Leu-Leu-Val-NH 2 (0.383 mmol, 1 equiv.), 0.33 g Fmoc-Glu(tBu)-OH (0.77 mmol, 2 equiv.), $0.10 \mathrm{~g}$ HOBt (0.77 mmol, 2 equiv.), $0.24 \mathrm{~mL}$ DIC (1.53 mmol, 4 equiv.) and $2.31 \mathrm{~mL}$ of DMF to generate a concentration of $0.3 \mathrm{M}$. The coupling reaction was run overnight a negative ninhydrin test was used to confirm reaction completion. The reaction mixture was drained to afford Resin-O-Leu-Leu-ValGlu(tBu)-NHFmoc. The Fmoc group was then removed following the Fmoc Removal procedure to afford Resin-O-Leu-Leu-Val-Glu(tBu)-NH2. 


\section{Resin-O- Leu-Leu-Val-Glu(tBu)-Pro--NH2}

Resin-O-Leu-Leu-Val-Glu(tBu)-Pro- $\mathrm{NH}_{2}$ was synthesized according to the Coupling Reaction procedure, using Resin-O-Leu-Leu-Val-Glu(tBu)-NH $\mathrm{NH}_{2}$ (0.383 mmol, 1 equiv.), 0.26 g Fmoc-Pro-OH (0.77 mmol, 2 equiv.), $1.28 \mathrm{~mL}$ HOAt (0.77 mmol, 2 equiv.), $0.24 \mathrm{~mL}$ DIC (1.53 mmol, 4 equiv.) and $1.03 \mathrm{~mL}$ of DMF to generate a concentration of $0.3 \mathrm{M}$. The coupling reaction was run overnight and a negative ninhydrin test was used to confirm reaction completion. The reaction mixture was drained to afford Resin-O-LeuLeu-Val-Glu(tBu)-Pro-NHFmoc. The Fmoc group was then removed following the Fmoc Removal procedure to afford Resin-O-Leu-Leu-Val-Glu(tBu)-Pro- $\mathrm{NH}_{2}$.

\section{Resin-O- Leu-Leu-Val-Glu(tBu)-Pro-Thr(tBu)-NH2}

Resin-O-Leu-Leu-Val-Glu(tBu)-Pro- $\mathrm{Thr}(\mathrm{tBu})-\mathrm{NH}_{2}$ was synthesized according to the Coupling Reaction procedure, using Resin-O-Leu-Leu-Val-Glu(tBu)-Pro-NH2 (0.383 mmol, 1 equiv.), 0.30 g FmocThr(tBu)-OH (0.77 mmol, 2 equiv.), 1.28 mL HOAt (0.77 mmol, 2 equiv.), 0.24 mL DIC (1.53 mmol, 4 equiv.) and $1.03 \mathrm{~mL}$ of DMF to generate a concentration of $0.3 \mathrm{M}$. The coupling reaction was run overnight and a negative ninhydrin test was used to confirm reaction completion. The reaction mixture was drained and a small portion of resin-bound peptide was cleaved with TFE: $\mathrm{CH}_{2} \mathrm{Cl}_{2}(1: 1 \mathrm{v} / \mathrm{v})$, filtered and the filtrate was dried under a stream of nitrogen. The sample was redissolved in acetonitrile/milli-Q water $(1: 1 \mathrm{v} / \mathrm{v})$ at a concentration of $1 \mathrm{mg} / \mathrm{ml}$ and was further diluted to $50 \mathrm{ppm}$. The sample was analysed by LC/MS where the reaction was determined to be completed by a change in the $\mathrm{m} / \mathrm{z}$ of the major product. The Fmoc group on Resin-O-Leu-Leu-Val-Glu(tBu)-Pro-Thr(tBu)-NHFmoc was then removed following the Fmoc Removal procedure to afford Resin-O-Leu-Leu-Val-Glu(tBu)-Pro-Thr(tBu)- $\mathrm{NH}_{2}$.

\section{Resin-O- Leu-Leu -Val-Glu(tBu)-Pro-Thr(tBu)-Thr(tBu)-NH2}

Resin-O-Leu-Leu-Val-Glu(tBu)-Pro-Thr(tBu)-Thr(tBu)-NH 2 was synthesized according to the Coupling Reaction procedure, using Resin-O-Leu-Leu-Val-Glu(tBu)-Thr(tBu)- $\mathrm{NH}_{2}(0.383 \mathrm{mmol}, 1$ equiv.), $0.30 \mathrm{~g}$ Fmoc-Thr(tBu)-OH (0.77 mmol, 2 equiv.), 1.28 mL HOAt (0.77 mmol, 2 equiv.), 0.24 mL DIC (1.53 
mmol, 4 equiv.) and $1.03 \mathrm{~mL}$ of DMF to generate a concentration of $0.3 \mathrm{M}$. The coupling reaction was run overnight and a negative ninhydrin test was used to confirm reaction completion. The reaction mixture was drained to afford Resin-O-Leu-Leu-Val-Glu(tBu)-Pro-Thr(tBu)-Thr(tBu)-NHFmoc. The Fmoc group was then removed following the Fmoc Removal procedure to afford Resin-O-Leu-Leu-ValGlu(tBu)-Pro-Thr(tBu)-Thr(tBu)- $\mathrm{NH}_{2}$.

\section{Resin-O- Leu-Leu -Val-Glu(tBu)-Pro-Thr(tBu)-Thr(tBu)-Asn(Trt)-NH2}

Resin-O-Leu-Leu-Val-Glu(tBu)-Pro-Thr(tBu)-Thr(tBu)-Asn(Trt)- $\mathrm{NH}_{2}$ was synthesized according to the Coupling Reaction procedure, using Resin-O-Leu-Leu-Val-Glu(tBu)-Thr(tBu)-Thr(tBu)- $\mathrm{NH}_{2}(0.383$ mmol, 1 equiv.), 0.46 g Fmoc-Asn(Trt)-OH (0.77 mmol, 2 equiv.), 1.28 mL HOAt (0.77 mmol, 2 equiv.), $0.24 \mathrm{~mL}$ DIC (1.53 mmol, 4 equiv.) and $1.03 \mathrm{~mL}$ of DMF to generate a concentration of $0.3 \mathrm{M}$. The coupling reaction was run overnight and a negative ninhydrin test was used to confirm reaction completion. The reaction mixture was drained to afford Resin-O-Leu-Leu-Val-Glu(tBu)-Pro-Thr(tBu)Thr(tBu)-Asn(Trt)-NHFmoc. The Fmoc group was then removed following the Fmoc Removal procedure to afford Resin-O-Leu-Leu-Val-Glu(tBu)-Pro-Thr(tBu)-Thr(tBu)-Asn(Trt)- $\mathrm{NH}_{2}$.

\section{Resin-O- Leu-Leu -Val-Glu(tBu)-Pro-Thr(tBu)-Thr(tBu)-Asn(Trt)-Phe-NH2}

Resin-O-Leu-Leu-Val-Glu(tBu)-Pro-Thr(tBu)-Thr(tBu)-Asn(Trt)-Phe- $\mathrm{NH}_{2}$ was synthesized according to the Coupling Reaction procedure, using Resin-O-Leu-Leu-Val-Glu(tBu)-Thr(tBu)-Asn(Trt)- $\mathrm{NH}_{2}(0.383$ mmol, 1 equiv.), 0.30 g Fmoc-Phe-OH (0.77 mmol, 2 equiv.), 1.28 mL HOAt (0.77 mmol, 2 equiv.), 0.24 $\mathrm{mL}$ DIC (1.53 mmol, 4 equiv.) and $1.03 \mathrm{~mL}$ of DMF to generate a concentration of $0.3 \mathrm{M}$. The coupling reaction was run overnight and a negative ninhydrin test was used to confirm reaction completion. The reaction mixture was drained to afford Resin-O-Leu-Leu-Val-Glu(tBu)-Pro-Thr(tBu)-Thr(tBu)-Asn(Trt)Phe-NHFmoc. The Fmoc group was then removed following the Fmoc Removal procedure to afford Resin-O-Leu-Leu-Val-Glu(tBu)-Pro-Thr(tBu)-Thr(tBu)-Asn(Trt)-Phe-NH2. 


\section{Resin-O- Leu-Leu -Val-Glu(tBu)-Pro-Thr(tBu)-Thr(tBu)-Asn(Trt)-Phe-Ser(tBu)-NH2}

Resin-O-Leu-Leu-Val-Glu(tBu)-Pro-Thr(tBu)-Thr(tBu)-Asn(Trt)-Phe-Ser(tBu)- $\mathrm{NH}_{2}$ was synthesized according to the Coupling Reaction procedure, using Resin-O-Leu-Leu-Val-Glu(tBu)-Thr(tBu)Asn(Trt)-NH 2 (0.383 mmol, 1 equiv.), 0.29 g Fmoc-Thr(tBu)-OH (0.77 mmol, 2 equiv.), 1.28 mL HOAt (0.77 mmol, 2 equiv.), $0.24 \mathrm{~mL}$ DIC (1.53 mmol, 4 equiv.) and $1.03 \mathrm{~mL}$ of DMF to generate a concentration of $0.3 \mathrm{M}$. The coupling reaction was run overnight and a negative ninhydrin test was used to confirm reaction completion. The reaction mixture was drained to afford Resin-O-Leu-Leu-ValGlu(tBu)-Pro-Thr(tBu)-Thr(tBu)-Asn(Trt)-Phe-Ser(tBu)-NHFmoc. The Fmoc group was then removed following the Fmoc Removal procedure to afford Resin-O-Leu-Leu-Val-Glu(tBu)-Pro-Thr(tBu)$\operatorname{Thr}(\mathrm{tBu})-\mathrm{Asn}(\mathrm{Trt})-\mathrm{Phe}-\mathrm{Ser}(\mathrm{tBu})-\mathrm{NH}_{2}$.

\section{HO- Leu-Leu -Val-Glu(tBu)-Pro-Thr(tBu)-Thr(tBu)-Asn(Trt)-Phe-Ser(tBu)-NH2}

The protected linear peptide HO- Leu-Leu -Val-Glu(tBu)-Pro-Thr(tBu)-Thr(tBu)-Asn(Trt)-Phe$\mathrm{Ser}(\mathrm{tBu})-\mathrm{NH}_{2}$ was generated following the Resin Cleavage procedure. The linear peptide was cleaved from the resin using a solution of $4.5 \mathrm{~mL}$ of trifluoroethanol and $4.5 \mathrm{~mL}$ of $\mathrm{CH}_{2} \mathrm{Cl}_{2}$. The resin containing solution was filtered and dried in vacuo to yield the protected linear pentapeptide as a pale-yellow solid (407 mg, 67\% overall).

\section{HO- Leu-Leu -Val-Glu-Pro-Thr-Thr-Asn-Phe-Ser-NH 2 (SP)}

The deprotected linear peptide HO-Leu-Leu-Val-Glu-Pro-Thr-Thr-Asn-Phe-Ser- $\mathrm{NH}_{2}$ was generated following the Global Deprotection procedure using 0.20 g HO-Leu-Leu-Val-Glu-Pro-Thr(tBu)-Thr(tBu)Asn(Trt)-Phe-Ser(tBu)-NH$(0.13$ mmol, 1 equiv. $), 0.87 \mathrm{~mL}$ of a mixed solution of TFA and $\mathrm{CH}_{2} \mathrm{Cl}_{2}(9: 1$ $\mathrm{v} / \mathrm{v}$ ) and $0.14 \mathrm{~mL}$ anisole (1.24 mmol, 10 equiv.) to remove the side chain protecting groups. The reaction mixture was stirred overnight and monitored via LC/MS. Upon completion, the reaction mixture was worked up as described in the Global Deprotection procedure to afford the crude linear peptide. The crude product was purified using HPLC, then lyophilised to generate pure compound as a white solid (32.3 mg, 
$22 \%$ overall). The purity of the final compound was found to be $>95 \%$ by ${ }^{1} \mathrm{H}$ NMR, $2-\mathrm{D}$ NMR (COSY, HSQC, HMBC) and LC/MS.

LC/MS (ESI) $m / z$ : $[\mathrm{M}+\mathrm{H}]^{+}$calculated for $\mathrm{C}_{51} \mathrm{H}_{81} \mathrm{~N}_{11} \mathrm{O}_{17}{ }^{+}, 1120.58$; found 1120.60 .

${ }^{1} \mathrm{H}$ NMR (600 MHz, DMSO-d6): $\delta 8.48(\mathrm{~d}, J=7.62 \mathrm{~Hz}, 1 \mathrm{H}, \mathrm{NH}), 8.13(\mathrm{~d}, J=6.20 \mathrm{~Hz}, 1 \mathrm{H}, \mathrm{NH}), 7.97$ $(\mathrm{d}, J=8.32 \mathrm{~Hz}, 1 \mathrm{H}, \mathrm{NH}), 7.88-7.83(\mathrm{~m}, 2 \mathrm{H}, \mathrm{NH}), 7.69(\mathrm{~d}, J=8.50 \mathrm{~Hz}, 1 \mathrm{H}, \mathrm{NH}), 7.64(\mathrm{~d}, J=8.68 \mathrm{~Hz}$, 1H, NH), 7.43 (s, 1H, NH), 7.25-7.15 (m, 5H, ArH Phe), 6.95 (s, 1H, NH), 4.61 (dd, J = 6.49, 13.87 Hz, 1H, $\alpha \mathrm{CH}$ Asp), 4.58-4.54 (m, 1H, $\alpha \mathrm{CH}$ Val), 4.39-4.35 (t, $J=6.97 \mathrm{~Hz}, 1 \mathrm{H}, \alpha \mathrm{CH}$ Thr), 4.35-4.28 (m, 1H, $\alpha \mathrm{CH}$ Thr), 4.35-4.28 (m, 1H, $\alpha \mathrm{CH}$ Glu), 4.28-4.24 (m, 1H, $\alpha \mathrm{CH}$ Leu), 4.21 (dd, $J=6.33,14.03 \mathrm{~Hz}, 1 \mathrm{H}$, $\alpha \mathrm{CH}$ Pro), 4.16-4.11 (m, 1H, $\alpha \mathrm{CH}$ Ser), 4.16-4.11 (m, 1H, $\alpha \mathrm{CH}$ Leu), 4.06-4.00 (m, 1H, $\beta \mathrm{CH}$ Thr), 3.88 (quin, $J=6.49 \mathrm{~Hz}, 1 \mathrm{H}, \beta \mathrm{CH}$ Thr), 3.76-3.70 \& 3.67-3.61 (m, $2 \mathrm{H}, \delta \mathrm{CH}_{2}$ Pro), 3.49-3.43 \& 3.32-3.26 (m, $\left.2 \mathrm{H}, \beta \mathrm{CH}_{2} \mathrm{Ser}\right), 3.32-3.26$ (m, $\left.1 \mathrm{H}, \alpha \mathrm{CH} \mathrm{Phe}\right), 3.07-3.02$ (dd, $J=9.84,14.01 \mathrm{~Hz}, 1 \mathrm{H}, \beta \mathrm{CH}_{2} \mathrm{Phe}$ ), 2.85$2.78\left(\mathrm{dd}, J=4.77,13.72 \mathrm{~Hz}, 1 \mathrm{H}, \beta \mathrm{CH}_{2} \mathrm{Phe}\right), 2.63-2.57 \& 2.46-2.41\left(\mathrm{~m}, 2 \mathrm{H}, \beta \mathrm{CH}_{2} \mathrm{Asp}\right), 2.28-2.15$ (m, $2 \mathrm{H}, \gamma \mathrm{CH}_{2} \mathrm{Glu}$ ), 2.05-1.69 (m, 2H, $\beta \mathrm{CH}_{2}$ Pro), 1.95 (dd, $J=6.88,13.47 \mathrm{~Hz}, 1 \mathrm{H}, \beta \mathrm{CH}$ Val), 2.05-1.69 (m, $\left.2 \mathrm{H}, \beta \mathrm{CH}_{2} \mathrm{Glu}\right), 2.05-1.69$ (m, 2H, $\gamma \mathrm{CH}_{2}$ Pro), 1.66-1.55 (m, 2H, $\gamma \mathrm{CH}$ Leu), 1.54-1.41 (m, 4H, $\beta \mathrm{CH}_{2} \mathrm{Leu}$ ), $1.12\left(\mathrm{~d}, J=6.14 \mathrm{~Hz}, 3 \mathrm{H}, \gamma \mathrm{CH}_{3} \mathrm{Thr}\right), 1.01$ (d, $\left.J=6.38 \mathrm{~Hz}, 3 \mathrm{H}, \gamma \mathrm{CH}_{3} \mathrm{Thr}\right), 0.89-0.85$ (m, 3H, $\left.\gamma \mathrm{CH}_{3} \mathrm{Val}\right)$, 0.83-0.77 (m, 3H, $\left.\gamma \mathrm{CH}_{3} \mathrm{Val}\right), 0.83-0.77\left(\mathrm{~m}, 12 \mathrm{H}, \delta \mathrm{CH}_{3} \mathrm{Leu}\right)$.

\section{Experimental Procedures for Compound 1}

\section{Resin-O-Phe- $\mathrm{NH}_{2}$}

The resin bound amino acid Resin-O-Phe- $\mathrm{NH}_{2}$ was synthesized according to the Resin Loading procedure. A sample of 2-ClTrt $\mathrm{Cl}$ resin $(0.6 \mathrm{~g}, 1.14 \mathrm{mmol} / \mathrm{g}$ loading, 1 equiv.) was placed in a reaction vessel and swelled with $\mathrm{CH}_{2} \mathrm{Cl}_{2}$ for 30 minutes then drained. To the swelled resin, $0.80 \mathrm{~g}$ Fmoc-Phe-OH (2.05 mmol, 3 equiv.) that was pre-dissolved in the minimum volume of DIPEA in $\mathrm{CH}_{2} \mathrm{Cl}_{2}(0.40 \mathrm{M})$ was added and the reaction was shaken at room temperature for $4 \mathrm{~h}$. The reaction mixture was drained and the resin was washed according to the Resin Loading protocol to produce Resin-O-Phe-NHFmoc. A sample 
of resin was removed and the resin loading was determined to be $0.493 \mathrm{mmol} / \mathrm{g}$. The Fmoc group was then removed following the Fmoc Removal procedure to produce Resin-O-Phe- $\mathrm{NH}_{2}$.

\section{Resin-O-Phe-Pro- $\mathrm{NH}_{2}$}

Resin-O-Phe-Pro- $\mathrm{NH}_{2}$ was synthesized following the Coupling Reaction procedure, using $0.685 \mathrm{~g}$ ResinO-Phe- $\mathrm{NH}_{2}$ (0.34 mmol, 1 equiv.), 0.23 g Fmoc-Pro-OH (0.68 mmol, 2 equiv.), 1.13 mL HOAt (0.68 mmol, 2 equiv.), $0.21 \mathrm{~mL}$ DIC (1.35 mmol, 4 equiv.), and $0.91 \mathrm{~mL}$ of DMF to generate a concentration of $0.3 \mathrm{M}$. The coupling reaction was run for $2 \mathrm{~h}$ and a negative ninhydrin test was used to confirm reaction completion. The reaction mixture was drained to afford Resin-O-Phe-Pro-NHFmoc. The Fmoc group was then removed following the Fmoc Removal procedure to afford Resin-O-Phe-Pro- $\mathrm{NH}_{2}$.

\section{Resin-O-Phe-Pro-Asp(OtBu)- $\mathrm{NH}_{2}$}

Resin-O-Phe-Pro-Asp(OtBu)- $\mathrm{NH}_{2}$ was synthesized following the Coupling Reaction procedure, using Resin-O-Phe-Pro- $\mathrm{NH}_{2}$ from the previous reaction, $0.28 \mathrm{~g}$ Fmoc-Asp(OtBu)-OH (0.68 mmol, 2 equiv.), $1.13 \mathrm{~mL}$ HOAt (0.68 mmol, 2 equiv.), $0.21 \mathrm{~mL}$ DIC (1.35 mmol, 4 equiv.), and $0.91 \mathrm{~mL}$ of DMF to generate a concentration of $0.3 \mathrm{M}$. The coupling reaction was run for $2 \mathrm{~h}$ and a negative ninhydrin test was used to confirm reaction completion. The reaction mixture was drained to afford Resin-O-Phe-Pro$\mathrm{Asp}(\mathrm{OtBu})-\mathrm{NHFmoc}$. The Fmoc group was then removed following the Fmoc Removal procedure to afford Resin-O-Phe-Pro-Asp(OtBu)- $\mathrm{NH}_{2}$.

\section{Resin-O-Phe-Pro-Asp(OtBu)-Trp(Boc)- $\mathrm{NH}_{2}$}

Resin-O-Phe-Pro_Asp(OtBu)-Trp(Boc)- $\mathrm{NH}_{2}$ was synthesized following the Coupling Reaction procedure, using Resin-O-Phe-Pro-Asp $(\mathrm{OtBu})-\mathrm{NH}_{2}$ from the previous reaction, $0.36 \mathrm{~g}$ Fmoc-Trp(Boc)$\mathrm{OH}$ (0.68 mmol, 2 equiv.), $0.091 \mathrm{~g} \mathrm{HOBt} \mathrm{(0.68} \mathrm{mmol,} 2$ equiv.), $0.21 \mathrm{~mL}$ DIC (1.35 mmol, 4 equiv.), and $2.04 \mathrm{~mL}$ DMF to generate a concentration of $0.3 \mathrm{M}$. The coupling reaction was run for $2 \mathrm{~h}$ and a negative 
ninhydrin test was used to confirm reaction completion. The reaction mixture was drained to afford ResinO-Phe-Pro-Asp(OtBu)-Trp(Boc)-NHFmoc. The Fmoc group was then removed following the Fmoc Removal procedure to afford Resin-O-Phe-Pro-Asp(OtBu)-Trp(Boc)- $\mathrm{NH}_{2}$.

\section{HO-Phe-Pro-Asp(OtBu)-Trp(Boc)--NH}

The protected linear peptide HO-Phe-Pro-Asp(OtBu)-Trp(Boc)- $\mathrm{NH}_{2}$ was generated following the Resin Cleavage of Linear Peptide procedure. The linear peptide was cleaved from the resin using a solution of $3.4 \mathrm{~mL}$ trifluoroethanol and $3.4 \mathrm{~mL} \mathrm{CH}_{2} \mathrm{Cl}_{2}$. The resin containing solution was filtered and dried in vacuo to yield the protected linear peptide as a pale yellow solid (153 mg, 63\% overall).

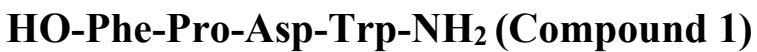

The deprotected linear peptide was synthesized following the Side Chain Deprotection procedure using 0.11 g crude HO-Phe-Pro-Asp(OtBu)-Trp(Boc)- $\mathrm{NH}_{2}(0.15 \mathrm{mmol}, 1$ equiv.), $0.55 \mathrm{~mL}$ of a mixed solution of TFA and $\mathrm{CH}_{2} \mathrm{Cl}_{2}(9: 1 \mathrm{v} / \mathrm{v})$ and $0.07 \mathrm{~mL}$ anisole $(0.61 \mathrm{mmol}, 4$ equiv.) to remove side chain protecting groups. The reaction mixture was stirred for $12 \mathrm{~h}$ and monitored via LC/MS. Upon completion, the reaction mixture was worked up as described in the Side Chain Deprotection procedure to afford the crude linear peptide. The crude product was purified using HPLC, then lyophilized to generate pure compound as a white solid $\left(54.5 \mathrm{mg}, 40 \%\right.$ overall). The purity of the final compound was found to be $>95 \%$ by ${ }^{1} \mathrm{H}$ NMR, 2-D NMR (COSY, HSQC, HMBC) and LC/MS.

LC/MS (ESI) $m / z:[\mathrm{M}+\mathrm{H}]^{+}$calculated for $\mathrm{C}_{29} \mathrm{H}_{33} \mathrm{~N}_{5} \mathrm{O}_{7}{ }^{+}$564.24, found 564.30.

${ }^{1} \mathrm{H}$ NMR (600 MHz, $\left.\mathrm{D}_{2} \mathrm{O}\right): \delta 7.56(\mathrm{~d}, J=7.98 \mathrm{~Hz}, 1 \mathrm{H}, \operatorname{ArH} \operatorname{Trp}), 7.50(\mathrm{~d}, J=8.16 \mathrm{~Hz}, 1 \mathrm{H}, \operatorname{ArH} \operatorname{Trp})$, 7.40-7.23 (m, 7H, ArH Trp, ArH Phe), 7.14 (t, J=7.5 Hz, 1H, ArH Trp), 4.83-4.81 (m, 1H, $\alpha \mathrm{H}$ Asp), 4.53-4.49 (m, 1H, $\alpha \mathrm{H}$ Phe), 4.27 (t, $J=7.44$ Hz, 1H, $\alpha \mathrm{H}$ Trp), 4.10 (dd, $J=3.96,1 \mathrm{H}, \alpha \mathrm{H}$ Pro), 3.53-3.49 (m, 1H, $\delta$ H Pro), 3.39-3.35 (m, 2H, $\beta$ H Trp), 3.31-3.25 (m, 1H, $\delta \mathrm{H} \mathrm{Pro),} \mathrm{3.21-2.98} \mathrm{(m,} \mathrm{2H,} \beta$ H Phe), 2.69- 
2.48 (m, 2H, $\beta \mathrm{H}$ Asp), 2.10-2.01 (m, 1H, $\beta \mathrm{H}$ Pro), 1.84-1.78 (m, 1H, $\gamma \mathrm{H}$ Pro), 1.76-1.72 (m, 1H, $\beta \mathrm{H}$ Pro), 1.69-1.61 (m, 1H, $\gamma \mathrm{H}$ Pro).

\section{Experimental Procedures for Compound 2}

\section{Resin-O-Phe- $\mathrm{NH}_{2}$}

The resin bound amino acid Resin-O-Phe- $\mathrm{NH}_{2}$ was synthesized according to the Resin Loading procedure. A sample of 2-ClTrt $\mathrm{Cl}$ resin $(0.5 \mathrm{~g}, 1.14 \mathrm{mmol} / \mathrm{g}$ loading, 1 equiv.) was placed in a reaction vessel and swelled with $\mathrm{CH}_{2} \mathrm{Cl}_{2}$ for 30 minutes then drained. To the resin, $0.66 \mathrm{~g}$ Fmoc-Phe-OH (1.7 mmol, 3 equiv.) that was pre-dissolved in the minimum amount of DIPEA in $\mathrm{CH}_{2} \mathrm{Cl}_{2}(0.40 \mathrm{M})$ was added and the reaction was shaken at room temperature for 4 hours. The reaction mixture was drained and washed according to the protocol to produce Resin-O-Phe-NHFmoc. A sample of resin was removed and the resin loading was determined to be $0.71 \mathrm{mmol} / \mathrm{g}$. The Fmoc group was then removed following the Fmoc Removal procedure to produce Resin-O-Phe- $\mathrm{NH}_{2}$.

\section{Resin-O-Phe-Pro-NH}

Resin-O-Phe-Pro- $\mathrm{NH}_{2}$ was synthesized following the Coupling Reaction procedure, using $0.5 \mathrm{~g}$ ResinO-Phe- $\mathrm{NH}_{2}$ (0.35 mmol, 1 equiv.), 0.24 g Fmoc-Pro-OH (0.71 mmol, 2 equiv.), 1.2 mL HOAt (0.71 mmol, 2 equiv.), $0.22 \mathrm{~mL}$ DIC (1.4 mmol, 4 equiv.), and $0.96 \mathrm{~mL}$ of DMF to generate a concentration of $0.3 \mathrm{M}$. The coupling reaction was run for 2 hours and a negative ninhydrin test was used to confirm reaction completion. The reaction mixture was drained to afford Resin-O-Phe-Pro-NHFmoc. The Fmoc group was then removed following the Fmoc Removal procedure to afford Resin-O-Phe-Pro- $\mathrm{NH}_{2}$.

\section{Resin-O-Phe-Pro-Asp(OtBu)-NH}

Resin-O-Phe-Pro-Asp $(\mathrm{OtBu})-\mathrm{NH}_{2}$ was synthesized following the Coupling Reaction procedure, using Resin-O-Phe-Pro- $\mathrm{NH}_{2}$ from the previous reaction, $0.29 \mathrm{~g}$ Fmoc-Asp(OtBu)-OH (0.71 mmol, 2 equiv.), 
$1.2 \mathrm{~mL}$ HOAt (0.71 mmol, 2 equiv.), $0.22 \mathrm{~mL}$ DIC (1.4 mmol, 4 equiv.), and $0.96 \mathrm{~mL}$ of DMF to generate a concentration of $0.3 \mathrm{M}$. The coupling reaction was run for 2 hours and a negative ninhydrin test was used to confirm reaction completion. The reaction mixture was drained to afford Resin-O-Phe-Pro$\mathrm{Asp}(\mathrm{OtBu})-\mathrm{NHFmoc}$. The Fmoc group was then removed following the Fmoc Removal procedure to afford Resin-O-Phe-Pro-Asp(OtBu)- $\mathrm{NH}_{2}$.

\section{Resin-O-Phe-Pro-Asp(OtBu)-Trp(Boc)-- $\mathrm{NH}_{2}$}

Resin-O-Phe-Pro_Asp(OtBu)-Trp(Boc)- $\mathrm{NH}_{2}$ was synthesized following the Coupling Reaction procedure, using Resin-O-Phe-Pro-Asp $(\mathrm{OtBu})-\mathrm{NH}_{2}$ from the previous reaction, $0.37 \mathrm{~g}$ Fmoc-Trp(Boc)$\mathrm{OH}$ (0.71 mmol, 2 equiv.), $1.2 \mathrm{~mL}$ HOAt ( $0.71 \mathrm{mmol}, 2$ equiv.), $0.22 \mathrm{~mL}$ DIC (1.4 mmol, 4 equiv.), and $0.96 \mathrm{~mL}$ DMF to generate a concentration of $0.3 \mathrm{M}$. The coupling reaction was run for 2 hours and a negative ninhydrin test was used to confirm reaction completion. The reaction mixture was drained to afford Resin-O-Phe-Pro-Asp(OtBu)-Trp(Boc)-NHFmoc. The Fmoc group was then removed following the Fmoc Removal procedure to afford Resin-O-Phe-Pro-Asp(OtBu)-Trp(Boc)- $\mathrm{NH}_{2}$.

\section{Resin-O-Phe-Pro-Asp(OtBu)-Trp(Boc)-Ser(OtBu)-NH}

Resin-O-Phe-Pro-Asp(OtBu)-Trp(Boc)-Ser(OtBu)- $\mathrm{NH}_{2}$ was synthesized following the Coupling Reaction procedure, using Resin-O-Phe-Pro-Asp $(\mathrm{OtBu})-\mathrm{Trp}(\mathrm{Boc})-\mathrm{NH}_{2}$ from the previous reaction, 0.27 g Fmoc-Ser(OtBu)-OH (0.71 mmol, 2 equiv.), 1.2 mL HOAt (0.71 mmol, 2 equiv.), 0.22 mL DIC (1.4 mmol, 4 equiv.), and $0.96 \mathrm{~mL} \mathrm{DMF}$ to generate a concentration of $0.3 \mathrm{M}$. The coupling reaction was run for 2 hours and a negative ninhydrin test was used to confirm reaction completion. The reaction mixture was drained to afford Resin-O-Phe-Pro-Asp(OtBu)-Trp(Boc)-Ser(OtBu)-NHFmoc. The Fmoc group was then removed following the Fmoc Removal procedure to afford Resin-O-Phe-Pro-Asp(OtBu)-Trp(Boc)$\operatorname{Ser}(\mathrm{OtBu})-\mathrm{NH}_{2}$. 


\section{HO-Phe-Pro-Asp(OtBu)-Trp(Boc)-Ser(OtBu)-NH}

The protected linear pentapeptide HO-Phe-Pro-Asp(OtBu)-Trp(Boc)-Ser(OtBu)- $\mathrm{NH}_{2}$ was generated following the Resin Cleavage of Linear Peptide procedure. The linear peptide was cleaved from the resin using a mixed solution of $3.5 \mathrm{~mL}$ trifluoroethanol and $3.5 \mathrm{~mL} \mathrm{CH}_{2} \mathrm{Cl}_{2}$. The resin containing solution was filtered and dried in vacuo to yield the protected linear pentapeptide as a pale yellow solid (287 $\mathrm{mg}$, overall $79 \%)$.

\section{HO-Phe-Pro-Asp-Trp-Ser-NH2 (Compound 2)}

The deprotected linear peptide was synthesized following the Side Chain Deprotection procedure using $0.25 \mathrm{~g}$ crude HO-Phe-Pro-Asp-Trp-Ser- $\mathrm{NH}_{2}$ (0.31 mmol, 1 equiv. $), 0.9 \mathrm{~mL}$ of a mixed solution of TFA and $\mathrm{CH}_{2} \mathrm{Cl}_{2}(9: 1 \mathrm{v} / \mathrm{v})$ and $0.2 \mathrm{~mL}$ anisole $(1.8 \mathrm{mmol}, 6$ equiv.) to generate the free side chains. The reaction mixture was stirred for 4 hours and monitored via LC/MS. Upon completion, the reaction mixture was worked up as described in the Side Chain Deprotection procedure and lyophilised to produce the crude linear peptide. The crude product was purified using HPLC to generate pure compound as a white solid (43 mg, 21\%). The purity of the final compound was found to be $>95 \%$ by ${ }^{1} \mathrm{H}$ NMR, 2-D NMR (COSY, HSQC, HMBC) and LC/MS.

LC/MS (ESI) $m / z:[\mathrm{M}+\mathrm{H}]^{+}$calculated for $\mathrm{C}_{32} \mathrm{H}_{39} \mathrm{~N}_{6} \mathrm{O}_{9}{ }^{+}, 651.28$; found 651.40 .

${ }^{1} \mathrm{H}$ NMR (600 MHz, D2O): $\delta 7.64(\mathrm{~d}, J=8.00 \mathrm{~Hz}, 1 \mathrm{H}, \operatorname{ArH} \operatorname{Trp}), 7.51$ (d, $\left.J=8.31 \mathrm{~Hz}, 1 \mathrm{H}, \operatorname{ArH} \operatorname{Trp}\right)$, 7.41-7.22 (m, 7H, ArH Trp, ArH Phe), 7.20-7.12 (m, 1H ArH Trp), 4.76 (d, 1H, $\alpha$ CH Asp), 4.51-4.37 (m, 1H, $\alpha \mathrm{CH}$ Trp), 4.17-4.02 (m, 2H, $\alpha \mathrm{CH}$ Pro, $\alpha \mathrm{H}$ Ser), 4.02-3.83 (m, 1H, $\alpha \mathrm{CH}$ Phe), 4.02-3.83 (m, $\left.1 \mathrm{H}, \beta \mathrm{CH}_{2} \mathrm{Ser}\right), 3.55-3.44 \& 3.32-3.21$ (m, $2 \mathrm{H}, \delta \mathrm{CH}_{2}$ Pro), 3.44-3.32 \& 3.32-3.21(m, $2 \mathrm{H}, \beta \mathrm{CH}_{2}$ Phe), 3.21-3.13 \& 3.05-2.93 (m, 2H, $\beta \mathrm{CH}_{2}$ Trp), 2.69-2.55 \& 2.51-2.35 (m, 2H, $\beta \mathrm{CH}_{2}$ Asp), 2.06-1.93 \&1.821.68 (m, 2H, $\gamma \mathrm{CH}_{2}$ Pro), $1.82-1.68$ \& 1.68-1.57 (m, $2 \mathrm{H}, \beta \mathrm{CH}_{2}$ Pro). 


\section{Experimental Procedures for Compound 3}

\section{Resin-O-Arg(Pbf)-NH}

The resin bound amino acid Resin-O-Arg(Pbf)- $\mathrm{NH}_{2}$ was synthesized according to the Resin Loading procedure. A sample of 2-ClTrt $\mathrm{Cl}$ resin $(0.7 \mathrm{~g}, 1.14 \mathrm{mmol} / \mathrm{g}$ loading, 1 equiv. $)$ was placed in a reaction vessel and swelled with $\mathrm{CH}_{2} \mathrm{Cl}_{2}$ for 30 minutes then drained. To the swelled resin, $1.55 \mathrm{~g}$ Fmoc- $\mathrm{Arg}(\mathrm{Pbf})-$ $\mathrm{OH}$ (2.39 mmol, 3 equiv.) that was predissolved in the minimum volume of DIPEA in $\mathrm{CH}_{2} \mathrm{Cl}_{2}(0.40 \mathrm{M})$ was added and the reaction was shaken at room temperature for $4 \mathrm{~h}$. The reaction mixture was drained and the resin was washed according to the Resin Loading protocol to produce Resin-O-Arg(Pbf)NHFmoc. A sample of resin was removed and the resin loading was determined to be $0.274 \mathrm{mmol} / \mathrm{g}$. The Fmoc group was then removed following the Fmoc Removal procedure to produce Resin-O-Arg(Pbf)$\mathrm{NH}_{2}$.

\section{Resin-O-Arg(Pbf)-Phe-NH}

Resin-O-Arg(Pbf)-Phe- $\mathrm{NH}_{2}$ was synthesized following the Coupling Reaction procedure, using $0.77 \mathrm{~g}$ Resin-O-Arg(Pbf)-NH $\mathrm{NH}_{2}$ (0.21 mmol, 1 equiv.), 0.16 g Fmoc-Phe-OH (0.42 mmol, 2 equiv.), $0.70 \mathrm{~mL}$ HOAt (0.42 mmol, 2 equiv.), $0.13 \mathrm{~mL}$ DIC (0.85 mmol, 4 equiv.), and $0.58 \mathrm{~mL}$ of DMF to generate a concentration of $0.3 \mathrm{M}$. The coupling reaction was run for $2 \mathrm{~h}$ and a negative ninhydrin test was used to confirm reaction completion. The reaction mixture was drained to afford Resin-O-Arg(Pbf)-PheNHFmoc. The Fmoc group was then removed following the Fmoc Removal procedure to afford Resin$\mathrm{O}-\mathrm{Arg}(\mathrm{Pbf})-\mathrm{Phe}-\mathrm{NH}_{2}$.

\section{Resin-O-Arg(Pbf)-Phe-Pro-NH}

Resin-O-Arg(Pbf)-Phe-Pro- $\mathrm{NH}_{2}$ was synthesized following the Coupling Reaction procedure, using Resin-O-Arg(Pbf)-Phe- $\mathrm{NH}_{2}$ from the previous reaction, $0.14 \mathrm{~g}$ Fmoc-Pro-OH (0.42 mmol, 2 equiv.), 0.70

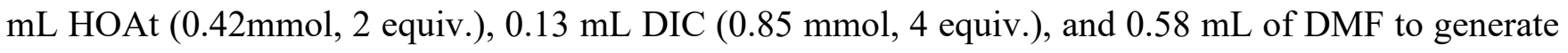


a concentration of $0.3 \mathrm{M}$. The coupling reaction was run for $2 \mathrm{~h}$ and a negative ninhydrin test was used to confirm reaction completion. The reaction mixture was drained to afford Resin-O-Arg(Pbf)-Phe-ProNHFmoc. The Fmoc group was then removed following the Fmoc Removal procedure to afford ResinResin-O-Arg(Pbf)-Phe-Pro- $\mathrm{NH}_{2}$.

\section{Resin-O-Arg(Pbf)-Phe-Pro-Asp(OtBu)--NH2}

Resin-O-Arg(Pbf)-Phe-Pro-Asp(OtBu)- $\mathrm{NH}_{2}$ was synthesized following the Coupling Reaction procedure, using Resin-O-Arg(Pbf)-Phe-Pro- $\mathrm{NH}_{2}$ from the previous reaction, $0.17 \mathrm{~g}$ Fmoc-Asp(OtBu)OH (0.42 mmol, 2 equiv.), $0.70 \mathrm{~mL}$ HOAt (0.42 mmol, 2 equiv.), $0.13 \mathrm{~mL}$ DIC (0.85 mmol, 4 equiv.), and $0.58 \mathrm{~mL}$ DMF to generate a concentration of $0.3 \mathrm{M}$. The coupling reaction was run for $2 \mathrm{~h}$ and a negative ninhydrin test was used to confirm reaction completion. The reaction mixture was drained to afford Resin-O-Arg(Pbf)-Phe-Pro-Asp(OtBu)-NHFmoc. The Fmoc group was then removed following the Fmoc Removal procedure to afford Resin-O-Arg(Pbf)-Phe-Pro-Asp(OtBu)- $\mathrm{NH}_{2}$.

\section{Resin-O-Arg(Pbf)-Phe-Pro-Asp(OtBu)-Trp(Boc)-NH}

Resin-O-Arg(Pbf)-Phe-Pro-Asp(OtBu)-Trp(Boc)- $\mathrm{NH}_{2}$ was synthesized following the Coupling Reaction procedure, using Resin-O-Arg( $\mathrm{Pbf})-\mathrm{Phe}-\mathrm{Pro}-\mathrm{Asp}(\mathrm{OtBu})-\mathrm{NH}_{2}$ from the previous reaction, $0.22 \mathrm{~g}$ FmocTrp(Boc)-OH (0.42 mmol, 2 equiv.), 0.057 g HOBt (0.42 mmol, 2 equiv.), 0.13 mL DIC (0.85 mmol, 4 equiv.), and 1.28 mL DMF to generate a concentration of $0.3 \mathrm{M}$. The coupling reaction was run for $2 \mathrm{~h}$ and a negative ninhydrin test was used to confirm reaction completion. The reaction mixture was drained to afford Resin-O-Arg(Pbf)-Phe-Pro-Asp(OtBu)-Trp(Boc)-NHFmoc. The Fmoc group was then removed following the Fmoc Removal procedure to afford Resin-O-Arg(Pbf)-Phe-Pro-Asp(OtBu)-Trp(Boc)-NH ${ }_{2}$. 


\section{HO-Arg(Pbf)-Phe-Pro-Asp(OtBu)-Trp(Boc)-NH}

The protected linear peptide $\mathrm{HO}-\mathrm{Arg}(\mathrm{Pbf})-\mathrm{Phe}-\mathrm{Pro}-\mathrm{Asp}(\mathrm{OtBu})-\mathrm{Trp}(\mathrm{Boc})-\mathrm{NH}_{2}$ was generated following the Resin Cleavage of Linear Peptide procedure. The linear peptide was cleaved from the resin using a solution of $3.65 \mathrm{~mL}$ trifluoroethanol and $3.65 \mathrm{~mL} \mathrm{CH}_{2} \mathrm{Cl}_{2}$. The resin containing solution was filtered and dried in vacuo to yield the protected linear peptide as a pale yellow solid (152.4 $\mathrm{mg}, 64 \%$ overall)

\section{HO-Arg-Phe-Pro-Asp-Trp-NH2 (Compound 3)}

The deprotected linear peptide was synthesized following the Side Chain Deprotection procedure using 0.1 g crude $\mathrm{HO}-\mathrm{Arg}(\mathrm{Pbf})-\mathrm{Phe}-\mathrm{Pro}-\mathrm{Asp}(\mathrm{OtBu})-\mathrm{Trp}(\mathrm{Boc})-\mathrm{NH}_{2}(0.089 \mathrm{mmol}, 1$ equiv. $), 0.50 \mathrm{~mL}$ of a mixed solution of TFA and $\mathrm{CH}_{2} \mathrm{Cl}_{2}(9: 1 \mathrm{v} / \mathrm{v})$ and $0.06 \mathrm{~mL}$ anisole $(0.53 \mathrm{mmol}, 6$ equiv.) to remove side chain protecting groups. The reaction mixture was stirred for $6 \mathrm{~h}$ and monitored via LC/MS. Upon completion, the reaction mixture was worked up as described in the Side Chain Deprotection procedure to afford the crude linear peptide. The crude product was purified using HPLC, then lyophilized to generate pure compound as a white solid ( $42.3 \mathrm{mg}, 42 \%$ overall). The purity of the final compound was found to be $>95 \%$ by ${ }^{1} \mathrm{H}$ NMR, 2-D NMR (COSY, HSQC, HMBC) and LC/MS.

LC/MS (ESI) m/z: [M+H] $]^{+}$calculated for $\mathrm{C}_{35} \mathrm{H}_{45} \mathrm{~N}_{9} \mathrm{O}_{8}{ }^{+}$720.34, found 720.35 .

${ }^{1} \mathrm{H}$ NMR (600 MHz, D $\left.2 \mathrm{O}\right): \delta 7.57$ (d, J = 8.04 Hz, 1H, ArH Trp), 7.50 (d, J = 8.22 Hz, 1H, ArH Trp), 7.40-7.24 (m, 7H, ArH Trp, ArH Phe), 7.14 (t, J = 7.5 Hz, 1H, ArH Trp), 4.83 (t, J = 7.29 Hz, 1H, $\alpha \mathrm{H}$ Asp), 4.59 (dd, J = 5.82 Hz, 1H, $\alpha \mathrm{H}$ Phe), 4.28 (t, J = 7.44 Hz, 1H, $\alpha \mathrm{H}$ Trp), 4.17 (dd, J = 5.34 Hz, 1H, $\alpha \mathrm{H}$ Arg), 4.09 (dd, J = 3.93 Hz, 1H, $\alpha \mathrm{H}$ Pro), 3.57-3.53 (m, 1H, $\delta \mathrm{H}$ Pro), 3.37-3.32 (m, 3H, $\beta H$ Trp, $\delta H$ Pro), 3.24-3.14 (m, 3H, $\beta \mathrm{H}$ Phe, $\delta \mathrm{H}$ Arg), 3.02 (dd, J = 9.72 Hz, 1H, $\beta \mathrm{H}$ Phe), 2.67-2.44 (m, 2H, $\beta \mathrm{H}$ Asp), 2.11-2.00 (m, 1H, $\beta \mathrm{H}$ Pro), 1.85-1.75 (m, 2H, $\beta \mathrm{H}$ Arg, $\gamma \mathrm{H}$ Pro), 1.72-1.63 (m, 1H, $\beta \mathrm{H}$ Arg), 1.62-1.52 (m, 4H, $\gamma \mathrm{H}$ Pro, $\beta \mathrm{H}$ Pro, $\gamma \mathrm{H}$ Arg). 


\section{Experimental Procedures for Compound 4}

\section{Resin-O-Phe- $\mathrm{NH}_{2}$}

The resin bound amino acid Resin-O-Phe- $\mathrm{NH}_{2}$ was synthesized according to the Resin Loading procedure. A sample of 2-ClTrt $\mathrm{Cl}$ resin $\left(0.6 \mathrm{~g}, 1.14 \mathrm{mmol} / \mathrm{g}\right.$ loading, 1 equiv.) was swelled in $\mathrm{CH}_{2} \mathrm{Cl}_{2}$ for 30 minutes then drained. To the resin, $0.80 \mathrm{~g}$ Fmoc-Phe-OH (2.1 mmol, 3 equiv.) that was predissolved in the minimum amount of DIPEA in $\mathrm{CH}_{2} \mathrm{Cl}_{2}(0.40 \mathrm{M})$ was added and the reaction was shaken at room temperature for 4 hours. The reaction mixture was drained and washed according to the protocol to produce Resin-O-Phe-NHFmoc. A sample of resin was removed and the resin loading was determined to be $0.57 \mathrm{mmol} / \mathrm{g}$. The Fmoc group was then removed following the Fmoc Removal procedure to produce Resin-O-Phe- $\mathrm{NH}_{2}$.

\section{Resin-O-Phe-Pro-Asp(OtBu)-Trp(Boc)-Ala- $\mathrm{NH}_{2}$}

Resin-O-Phe-Pro-Asp(OtBu)-Trp(Boc)-Ala- $\mathrm{NH}_{2}$ was synthesized following the Coupling Reaction procedure, using $0.6 \mathrm{~g}$ Resin-O-Phe- $\mathrm{NH}_{2}(0.34 \mathrm{mmol}, 1$ equiv.), from the previous reaction, $0.35 \mathrm{~g}$ FmocPro-OH (1.0 mmol, 3 equiv.), 0.42 g Fmoc-Asp(OtBu)-OH (1.0 mmol, 3 equiv.), 0.54 g Fmoc-Trp(Boc)$\mathrm{OH}$ (1.0 mmol, 3 equiv.), 0.32 g Fmoc-Ala-OH (1.0 mmol, 3 equiv.), were sequentially coupled utilizing $1.1 \mathrm{~mL}$ HOAt (0.68 mmol, 2 equiv.), $0.21 \mathrm{~mL}$ DIC (1.4 mmol, 4 equiv.), and 2.1 mL DMF to generate a concentration of $0.3 \mathrm{M}$. The coupling reaction was run for 2 hours and a negative ninhydrin test was used to confirm reaction completion. The reaction mixture was drained to afford Resin-O-Phe-Pro-Asp(OtBu)Trp(Boc)-Ala-NHFmoc. The Fmoc group was then removed following the Fmoc Removal procedure to afford Resin-O-Phe-Pro-Asp(OtBu)-Trp(Boc)-Ala- $\mathrm{NH}_{2}$.

\section{HO-Phe-Pro-Asp(OtBu)-Trp(Boc)-Ala- $\mathrm{NH}_{2}$}

The protected linear pentapeptide HO-Phe-Pro-Asp(OtBu)-Trp(Boc)-Ala- $\mathrm{NH}_{2}$ was generated following the Resin Cleavage of Linear Peptide procedure. The linear peptide was cleaved from the resin using a 
mixed solution of $3.9 \mathrm{~mL}$ trifluoroethanol and $3.9 \mathrm{~mL} \mathrm{CH}_{2} \mathrm{Cl}_{2}$. The resin containing solution was filtered and dried in vacuo to yield the protected linear pentapeptide as a pale yellow solid (194 mg, overall 72\%).

\section{HO-Phe-Pro-Asp-Trp-Ala-NH 2 (Compound 4)}

The deprotected linear peptide was synthesized following the Side Chain Deprotection procedure using $0.19 \mathrm{~g}$ crude HO-Phe-Pro-Asp-Trp-Ala- $\mathrm{NH}_{2}(0.25 \mathrm{mmol}, 1$ equiv. $), 774 \mu \mathrm{L}$ of a mixed solution of TFA and $\mathrm{CH}_{2} \mathrm{Cl}_{2}(9: 1 \mathrm{v} / \mathrm{v})$ and $160 \mu \mathrm{L}$ anisole $(1.5 \mathrm{mmol}, 6$ equiv.) to generate the free side chains. The reaction mixture was stirred for 4 hours and monitored via LC/MS. Upon completion, the reaction mixture was worked up as described in the Side Chain Deprotection procedure and lyophilised to produce the crude linear peptide. The crude product was purified using HPLC to generate pure compound as a white solid $(34 \%)$.

LC/MS (ESI) $m / z$ : $[\mathrm{M}+\mathrm{H}]^{+}$calculated for $\mathrm{C}_{32} \mathrm{H}_{39} \mathrm{~N}_{6} \mathrm{O}_{8}{ }^{+}, 635.28$; found 635.35 . The purity of the final compound was found to be $>95 \%$ by ${ }^{1} \mathrm{H}$ NMR, 2-D NMR (COSY, HSQC, HMBC) and LC/MS.

${ }^{1} \mathrm{H}$ NMR (600 MHz, D2O): $\delta 7.64(\mathrm{~d}, \mathrm{~J}=7.91 \mathrm{~Hz}, 1 \mathrm{H}, \operatorname{ArH} \operatorname{Trp}), 7.50$ (d, J = 8.20 Hz, 1H, ArH Trp), 7.42-7.22 (m, 7H, ArH Trp, ArH Phe), 7.18-7.12 (m, 1H ArH Trp), 4.70 (d, 1H, $\alpha \mathrm{H}$ Asp), 4.50-4.38 (m, 1H, $\alpha \mathrm{H}$ Trp), 4.14-4.01 (m, 2H, $\alpha \mathrm{H}$ Phe, $\alpha \mathrm{H}$ Ala), 3.52-3.44 (m, 1H, $\beta \mathrm{H}$ Pro), 3.31-3.12 (m, 4H, $\beta \mathrm{H}$ Pro, $\beta \mathrm{H}$ Trp, $\delta \mathrm{H}$ Pro), 3.04-2.95 (m, 1H, $\beta \mathrm{H}$ Trp), 2.70-2.56 (m, 1H, $\beta \mathrm{H}$ Asp), 2.53-2.39 (m, 1H, $\beta \mathrm{H}$ Asp), 2.06-1.95 (m, 1H, $\gamma \mathrm{H}$ Pro), 1.81-1.71 (m, 2H, $\gamma \mathrm{H}$ Pro, $\beta \mathrm{H}$ Pro), 1.67-1.56 (m, 1H, $\beta \mathrm{H}$ Pro), $1.51(\mathrm{~d}, \mathrm{~J}=$ $7.10 \mathrm{~Hz}, 3 \mathrm{H}, \mathrm{Ala})$.

\section{Experimental Procedures for Compound 5}

\section{Resin-O-Phe- $\mathrm{NH}_{2}$}

The resin bound amino acid Resin-O-Phe- $\mathrm{NH}_{2}$ was synthesized according to the Resin Loading procedure. A sample of 2-ClTrt $\mathrm{Cl}$ resin (1 g, $1.14 \mathrm{mmol} / \mathrm{g}$ loading, 1 equiv.) was swelled in $\mathrm{CH}_{2} \mathrm{Cl}_{2}$ for 30 minutes then drained. To the resin, $1.33 \mathrm{~g}$ Fmoc-Phe-OH (3.4 mmol, 3 equiv.) that was pre-dissolved 
in the minimum amount of DIPEA in $\mathrm{CH}_{2} \mathrm{Cl}_{2}(0.40 \mathrm{M})$ was added and the reaction was shaken at room temperature for 4 hours. The reaction mixture was drained and washed according to the protocol to produce Resin-O-Phe-NHFmoc. A sample of resin was removed and the resin loading was determined to be $0.45 \mathrm{mmol} / \mathrm{g}$. The Fmoc group was then removed following the Fmoc Removal procedure to produce Resin-O-Phe- $\mathrm{NH}_{2}$.

\section{Resin-O-Phe-Pro-Asp(OtBu)-Ala-Ser(Trt)- $\mathrm{NH}_{2}$}

Resin-O-Phe-Pro-Asp(OtBu)-Ala-Ser(Trt)- $\mathrm{NH}_{2}$ was synthesized following the Coupling Reaction procedure, using 1 g Resin-O-Phe- $\mathrm{NH}_{2}$ (0.45 mmol, 1 equiv.), 0.31 g Fmoc-Pro-OH (0.90 mmol, 2 equiv.), 0.37 g Fmoc-Asp(OtBu)-OH (0.90 mmol, 2 equiv.), 0.17 g Fmoc-Ala-OH (0.54 mmol, 2 equiv.), 0.31 g Fmoc-Ser(Trt)-OH (0.54 mmol, 2 equiv.) were sequentially coupled utilizing 1.5 mL HOAt (0.90 mmol, 2 equiv.), $0.28 \mathrm{~mL}$ DIC (1.8 mmol, 4 equiv.), and $1.2 \mathrm{~mL}$ of DMF to generate a concentration of $0.3 \mathrm{M}$. The coupling reaction was run for 2 hours and a negative ninhydrin test was used to confirm reaction completion. The reaction mixture was drained to afford Resin-O-Phe-Pro-Asp(OtBu)-AlaSer(Trt)-NHFmoc. The Fmoc group was then removed following the Fmoc Removal procedure to afford Resin-O-Phe-Pro-Asp(OtBu)-Ala-Ser(Trt)- $\mathrm{NH}_{2}$.

\section{HO-Phe-Pro-Asp(OtBu)-Ala-Ser(Trt)-NH}

The protected linear pentapeptide HO-Phe-Pro-Asp(OtBu)-Ala-Ser(Trt)- $\mathrm{NH}_{2}$ was generated following the Resin Cleavage of Linear Peptide procedure. The linear peptide was cleaved from the resin using a mixed solution of $3.9 \mathrm{~mL}$ trifluoroethanol and $3.9 \mathrm{~mL} \mathrm{CH}_{2} \mathrm{Cl}_{2}$. The resin containing solution was filtered and dried in vacuo to yield the protected linear pentapeptide as a pale yellow solid (527 mg, overall 52\%). 


\section{HO-Phe-Pro-Asp-Ala-Ser-NH2 (Compound 5)}

The deprotected linear peptide was synthesized following the Side Chain Deprotection procedure using $0.12 \mathrm{~g}$ crude HO-Phe-Pro-Asp-Trp-Ser- $\mathrm{NH}_{2}$ (0.14 mmol, 1 equiv. $), 462 \mu \mathrm{L}$ of a mixed solution of TFA and $\mathrm{CH}_{2} \mathrm{Cl}_{2}(9: 1 \mathrm{v} / \mathrm{v})$ and $92 \mu \mathrm{L}$ anisole $(0.84 \mathrm{mmol}, 6$ equiv.) to generate the free side chains. The reaction mixture was stirred for 4 hours and monitored via LC/MS. Upon completion, the reaction mixture was worked up as described in the Side Chain Deprotection procedure and lyophilised to produce the crude linear peptide. The crude product was purified using HPLC to generate pure compound as a white solid $(4 \%)$.

LC/MS (ESI) $m / z$ : $[\mathrm{M}+\mathrm{H}]^{+}$calculated for $\mathrm{C}_{24} \mathrm{H}_{34} \mathrm{~N}_{5} \mathrm{O}_{9}{ }^{+}$, 536.23; found 536.35. The purity of the final compound was found to be $>95 \%$ by ${ }^{1} \mathrm{H}$ NMR, 2-D NMR (COSY, HSQC, HMBC) and LC/MS.

${ }^{1} \mathrm{H}$ NMR (600 MHz, $\left.\mathrm{D}_{2} \mathrm{O}\right): \delta$ 7.41-7.25 (m, 5H, ArH Phe), 4.86-4.82 (dd, J = 9.48 Hz, 1H, $\alpha \mathrm{H}$ Asp), 4.504.34 (m, 3H $\alpha \mathrm{H}$ Phe, $\alpha \mathrm{H}$ Ala, $\alpha \mathrm{H}$ Pro), 4.18-4.11 (m, 1H, $\alpha \mathrm{H}$ Ser), 4.07-3.91 (m, 2H, $\beta \mathrm{H}$ Ser), 3.84-3.72 (m, 1H, $\delta \mathrm{H}$ Pro), 3.72-3.60 (m, 1H, $\delta \mathrm{H}$ Pro), 3.28-3.13 (m, 1H, $\beta \mathrm{H}$ Phe), 3.13-2.94 (m, 1H, $\beta \mathrm{H}$ Phe), 2.74-2.61 (m, 1H, $\beta \mathrm{H} \mathrm{Asp),} \mathrm{2.55-2.46} \mathrm{(m,} \mathrm{1H,} \beta \mathrm{H} \mathrm{Asp),} \mathrm{2.25-2.10} \mathrm{(m,} \mathrm{1H,} \beta \mathrm{H}$ Pro), 2.10-1.93 (m, 1H, $\gamma \mathrm{H}$ Pro), 1.93-1.76 (m, 2H, $\gamma \mathrm{H}$ Pro, $\beta \mathrm{H}$ Pro), 1.40 (d, J = 7.26 Hz, 3H, Ala).

\section{Experimental Procedures for Compound 6}

\section{Resin-O-Phe- $\mathrm{NH}_{2}$}

The resin bound amino acid Resin-O-Phe- $\mathrm{NH}_{2}$ was synthesized according to the Resin Loading procedure. A sample of 2-ClTrt $\mathrm{Cl}$ resin (0.6 g, $1.14 \mathrm{mmol} / \mathrm{g}$ loading, 1 equiv.) was swelled in $\mathrm{CH}_{2} \mathrm{Cl}_{2}$ for 30 minutes then drained. To the resin, $0.80 \mathrm{~g}$ Fmoc-Phe-OH (2.1 mmol, 3 equiv.) that was predissolved in the minimum amount of DIPEA in $\mathrm{CH}_{2} \mathrm{Cl}_{2}(0.40 \mathrm{M})$ was added and the reaction was shaken at room temperature for 4 hours. The reaction mixture was drained and washed according to the protocol to produce Resin-O-Phe-NHFmoc. A sample of resin was removed and the resin loading was determined 
to be $0.35 \mathrm{mmol} / \mathrm{g}$. The Fmoc group was then removed following the Fmoc Removal procedure to produce Resin-O-Phe- $\mathrm{NH}_{2}$.

\section{Resin-O-Phe-Pro-Ala-Trp(Boc)-Ser(Trt)- $\mathrm{NH}_{2}$}

Resin-O-Phe-Pro-Ala-Trp(Boc)-Ser(Trt)- $\mathrm{NH}_{2}$ was synthesized following the Coupling Reaction procedure, using 0.6 g Resin-O-Phe- $\mathrm{NH}_{2}$ (0.48 mmol, 1 equiv.), 0.16 g Fmoc-Pro-OH (0.49 mmol, 2 equiv.), 0.15 g Fmoc-Ala-OH (0.49 mmol, 2 equiv.), 0.26 g Fmoc-Trp(Boc)-OH (0.49 mmol, 2 equiv.), 0.28 g Fmoc-Ser(Trt)-OH (0.49 mmol, 2 equiv.) were sequentially coupled utilizing $0.81 \mathrm{~mL}$ HOAt (0.49 mmol, 2 equiv.), $0.15 \mathrm{~mL}$ DIC (0.97 mmol, 4 equiv.), and $0.66 \mathrm{~mL}$ of DMF to generate a concentration of $0.3 \mathrm{M}$. The coupling reaction was run for 2 hours and a negative ninhydrin test was used to confirm reaction completion. The reaction mixture was drained to afford Resin-O-Phe-Pro-Ala-Trp(Boc)Ser(Trt)-NHFmoc. The Fmoc group was then removed following the Fmoc Removal procedure to afford Resin-O-Phe-Pro-Ala-Trp(Boc)-Ser(Trt)-NH $\mathrm{NH}_{2}$

\section{HO-Phe-Pro-Ala-Trp(Boc)-Ser(Trt)- $\mathrm{NH}_{2}$}

The protected linear pentapeptide HO-Phe-Pro-Asp-Ala-Trp(Boc)-Ser(Trt)- $\mathrm{NH}_{2}$ was generated following the Resin Cleavage of Linear Peptide procedure. The linear peptide was cleaved from the resin using a mixed solution of $6.4 \mathrm{~mL}$ trifluoroethanol and $6.4 \mathrm{~mL} \mathrm{CH}_{2} \mathrm{Cl}_{2}$. The resin containing solution was filtered and dried in vacuo to yield the protected linear pentapeptide as a pale yellow solid (372 $\mathrm{mg}$, overall 87\%).

\section{HO-Phe-Pro-Ala-Trp-Ser-NH (Compound 6) $^{-}$}

The deprotected linear peptide was synthesized following the Side Chain Deprotection procedure using $0.37 \mathrm{~g}$ crude HO-Phe-Pro-Ala-Trp-Ser- $\mathrm{NH}_{2}$ (0.39 mmol, 1 equiv.), $1.5 \mathrm{~mL}$ of a mixed solution of TFA and $\mathrm{CH}_{2} \mathrm{Cl}_{2}(9: 1 \mathrm{v} / \mathrm{v})$ and $250 \mu \mathrm{L}$ anisole $(2.4 \mathrm{mmol}, 6$ equiv.) to generate the free side chains. The reaction mixture was stirred for 4 hours and monitored via LC/MS. Upon completion, the reaction mixture was 
worked up as described in the Side Chain Deprotection procedure and lyophilised to produce the crude linear peptide. The crude product was purified using HPLC to generate pure compound as a white solid (27\%). The purity of the final compound was found to be $>95 \%$ by ${ }^{1} \mathrm{H}$ NMR, 2-D NMR (COSY, HSQC, $\mathrm{HMBC}$ ) and LC/MS.

LC/MS (ESI) $m / z$ : $[\mathrm{M}+\mathrm{H}]^{+}$calculated for $\mathrm{C}_{31} \mathrm{H}_{39} \mathrm{~N}_{6} \mathrm{O}_{7}{ }^{+}, 606.28$; found 607.55.

${ }^{1} \mathrm{H}$ NMR (600 MHz, $\left.\mathrm{D}_{2} \mathrm{O}\right): \delta 7.59(\mathrm{~d}, \mathrm{~J}=8.05 \mathrm{~Hz}, 1 \mathrm{H}$ ArH Trp), 7.53 (d, J = 8.24 Hz, 1H, ArH Trp), 7.42-7.09 (m, 8H, ArH Phe, ArH Trp), 4.65 (t, J = 7.64 Hz, 1H $\alpha \mathrm{H}$ Trp), 4.43-4.31 (m, 2H, $\alpha \mathrm{H}$ Phe, $\alpha \mathrm{H}$ Ala), 4.15-4.01 (m, 2H, $\alpha \mathrm{H}$ Ser, $\alpha \mathrm{H}$ Pro), 4.01-3.83 (m, 2H, $\beta \mathrm{H}$ Ser), 3.50-3.35 (m, 1H, $\delta \mathrm{H}$ Pro), 3.353.16 (m, 3H, $\delta$ H Pro, $\beta$ H Trp), 3.16-3.08 (m, 1H, $\beta$ H Phe), 3.08-2.97 (m, 1H, $\beta$ H Phe), 2.27-2.07 (m, 1H, $\gamma$ H Pro), 1.99-1.71 (m, 3H, $\gamma \mathrm{H}$ Pro, $\beta \mathrm{H}$ Pro), 1.24 (d, J = 7.09 Hz, 3H, Ala).

\section{Experimental Procedures for Compound 7}

\section{Resin-O-Phe- $\mathrm{NH}_{2}$}

The resin bound amino acid Resin-O-Phe- $\mathrm{NH}_{2}$ was synthesized according to the Resin Loading procedure. A sample of 2-ClTrt $\mathrm{Cl}$ resin (0.8 g, $1.14 \mathrm{mmol} / \mathrm{g}$ loading, 1 equiv.) was swelled in $\mathrm{CH}_{2} \mathrm{Cl}_{2}$ for 30 minutes then drained. To the resin, $1.1 \mathrm{~g}$ Fmoc-Phe-OH (2.8 mmol, 3 equiv.) that was pre-dissolved in the minimum amount of DIPEA in $\mathrm{CH}_{2} \mathrm{Cl}_{2}(0.40 \mathrm{M})$ was added and the reaction was shaken at room temperature for 4 hours. The reaction mixture was drained and washed according to the protocol to produce Resin-O-Phe-NHFmoc. A sample of resin was removed and the resin loading was determined to be $0.71 \mathrm{mmol} / \mathrm{g}$. The Fmoc group was then removed following the Fmoc Removal procedure to produce Resin-O-Phe- $\mathrm{NH}_{2}$.

\section{Resin-O-Phe-Ala-Asp(OtBu)-Trp(Boc)-Ser(Trt)-NH}

Resin-O-Phe-Ala-Asp(OtBu)-Trp(Boc)-Ser(Trt)- $\mathrm{NH}_{2}$ was synthesized following the Coupling Reaction procedure, using 0.8 g Resin-O-Phe- $\mathrm{NH}_{2}$ (0.58 mmol, 1 equiv.), 0.36 g Fmoc-Ala-OH (1.2 mmol, 2 
equiv.), 0.47 g Fmoc-Asp(OtBu)-OH (1.2 mmol, 2 equiv.), 0.61 g Fmoc-Trp(Boc)-OH (1.2 mmol, 2 equiv.), 0.66 g Fmoc-Ser(Trt)-OH (1.2 mmol, 2 equiv.) were sequentially coupled utilizing 1.9 mL HOAt (1.2 mmol, 2 equiv.), $0.36 \mathrm{~mL}$ DIC (2.3 mmol, 4 equiv.), and $1.6 \mathrm{~mL}$ of DMF to generate a concentration of $0.3 \mathrm{M}$. The coupling reaction was run for 2 hours and a negative ninhydrin test was used to confirm reaction completion. The reaction mixture was drained to afford Resin-O-Phe-Ala-Asp(OtBu)-Trp(Boc)Ser(Trt)-NHFmoc. The Fmoc group was then removed following the Fmoc Removal procedure to afford Resin-O-Phe-Ala-Asp(OtBu)-Trp(Boc)-Ser(Trt)- $\mathrm{NH}_{2}$.

\section{HO-Phe-Ala-Asp(OtBu)-Trp(Boc)-Ser(Trt)-NH}

The protected linear pentapeptide HO-Phe-Ala-Asp(OtBu)-Trp(Boc)-Ser(Trt)- $\mathrm{NH}_{2}$ was generated following the Resin Cleavage of Linear Peptide procedure. The linear peptide was cleaved from the resin using a mixed solution of $6.8 \mathrm{~mL}$ trifluoroethanol and $6.8 \mathrm{~mL} \mathrm{CH}_{2} \mathrm{Cl}_{2}$. The resin containing solution was filtered and dried in vacuo to yield the protected linear pentapeptide as a pale yellow solid (472 $\mathrm{mg}$, overall $80 \%$ ).

\section{HO-Phe-Ala-Asp-Trp-Ser-NH2 (Compound 7)}

The deprotected linear peptide was synthesized following the Side Chain Deprotection procedure using $0.47 \mathrm{~g}$ crude HO-Phe-Ala-Asp-Trp-Ser- $\mathrm{NH}_{2}$ (0.46 mmol, 1 equiv.), $1.9 \mathrm{~mL}$ of a mixed solution of TFA and $\mathrm{CH}_{2} \mathrm{Cl}_{2}(9: 1 \mathrm{v} / \mathrm{v})$ and $301 \mu \mathrm{L}$ anisole $(2.8 \mathrm{mmol}, 6$ equiv. $)$ to generate the free side chains. The reaction mixture was stirred for 4 hours and monitored via LC/MS. Upon completion, the reaction mixture was worked up as described in the Side Chain Deprotection procedure and lyophilised to produce the crude linear peptide. The crude product was purified using HPLC to generate pure compound as a white solid (38\%). The purity of the final compound was found to be $>95 \%$ by ${ }^{1} \mathrm{H}$ NMR, 2 -D NMR (COSY, HSQC, HMBC) and LC/MS.

LC/MS (ESI) $m / z$ : $[\mathrm{M}+\mathrm{H}]^{+}$calculated for $\mathrm{C}_{30} \mathrm{H}_{37} \mathrm{~N}_{6} \mathrm{O}_{9}{ }^{+}, 625.25$; found 625.60 . 
${ }^{1} \mathrm{H}$ NMR (600 MHz, D $\left.2 \mathrm{O}\right): \delta 7.63(\mathrm{~d}, \mathrm{~J}=7.93 \mathrm{~Hz}, 1 \mathrm{H}, \operatorname{ArH} \operatorname{Trp}), 7.50$ (d, J = 8.09 Hz, 1H, ArH Trp), 7.40-7.21 (m, 7H, ArH Trp, ArH Phe), 7.21-7.11 (m, 1H, ArH Trp), 4.71 (t, J = 7.29 Hz, 1H, $\alpha \mathrm{H}$ Trp), 4.54-4.40 (m, 2H, $\alpha \mathrm{H}$ Asp, $\alpha \mathrm{H}$ Phe), 4.18-4.05 (m, 2H $\alpha \mathrm{H}$ Ala, $\alpha \mathrm{H}$ Ser), 4.01-3.90 (m, 2H, $\beta \mathrm{H} \mathrm{Ser),} \mathrm{3.35-}$ 3.23 (m, 2H, $\beta \mathrm{H}$ Trp), 3.23-3.13 (m, 1H, $\beta \mathrm{H}$ Phe), 3.04-2.94 (m, 1H, $\beta \mathrm{H}$ Phe), 2.49 (d, J = 6.67 Hz, 2H, $\beta \mathrm{H}$ Asp), 1.21 (d, J = 7.18 Hz, 3H Ala).

\section{Experimental Procedures for Compound 8}

\section{Resin-O-Ala- $\mathrm{NH}_{2}$}

The resin bound amino acid Resin-O-Phe- $\mathrm{NH}_{2}$ was synthesized according to the Resin Loading procedure. A sample of 2-ClTrt $\mathrm{Cl}$ resin ( $0.6 \mathrm{~g}, 1.14 \mathrm{mmol} / \mathrm{g}$ loading, 1 equiv.) was swelled with $\mathrm{CH}_{2} \mathrm{Cl}_{2}$ for 30 minutes then drained. To the resin, $0.73 \mathrm{~g}$ Fmoc-Ala-OH (2.3 mmol, 3 equiv.) that was predissolved in the minimum amount of DIPEA in $\mathrm{CH}_{2} \mathrm{Cl}_{2}(0.40 \mathrm{M})$ was added and the reaction was shaken at room temperature for 4 hours. The reaction mixture was drained and washed according to the protocol to produce Resin-O-Phe-NHFmoc. A sample of resin was removed and the resin loading was determined to be $0.7 \mathrm{mmol} / \mathrm{g}$. The Fmoc group was then removed following the Fmoc Removal procedure to produce Resin-O-Ala- $\mathrm{NH}_{2}$.

\section{Resin-O-Ala-Pro-Asp(OtBu)-Trp(Boc)-Ser(OtBu)-NH}

Resin-O-Ala-Pro-Asp(OtBu)-Trp(Boc)-Ser(OtBu)- $\mathrm{NH}_{2}$ was synthesized following the Coupling Reaction procedure, using 0.6 g Resin-O-Ala- $\mathrm{NH}_{2}$ (0.48 mmol, 1 equiv.), 0.32 g Fmoc-Pro-OH (0.96 mmol, 2 equiv.), 0.39 g Fmoc-Asp(OtBu)-OH (0.96 mmol, 2 equiv.), 0.50 g Fmoc-Trp(Boc)-OH (0.96 mmol, 2 equiv.), 0.37 g Fmoc-Ser(OtBu)-OH (0.96 mmol, 2 equiv.) were sequentially coupled utilizing 1.6 mL HOAt (0.96 mmol, 2 equiv.), $0.30 \mathrm{~mL}$ DIC (1.9 mmol, 4 equiv.), and $1.3 \mathrm{~mL}$ of DMF to generate a concentration of $0.3 \mathrm{M}$. The coupling reaction was run for 2 hours and a negative ninhydrin test was used to confirm reaction completion. The reaction mixture was drained to afford Resin-O-Ala-Pro- 
$\mathrm{Asp}(\mathrm{OtBu})-\mathrm{Trp}(\mathrm{Boc})-\mathrm{Ser}(\mathrm{OtBu})-\mathrm{NHFmoc}$. The Fmoc group was then removed following the Fmoc Removal procedure to afford Resin-O-Ala-Pro-Asp(OtBu)-Trp(Boc)-Ser(OtBu)- $\mathrm{NH}_{2}$.

\section{HO-Ala-Pro-Asp(OtBu)-Trp(Boc)-Ser(OtBu)-- $\mathrm{NH}_{2}$}

The protected linear pentapeptide HO-Ala-Pro-Asp(OtBu)-Trp(Boc)-Ser(OtBu)- $\mathrm{NH}_{2}$ was generated following the Resin Cleavage of Linear Peptide procedure. The linear peptide was cleaved from the resin using a mixed solution of $4.9 \mathrm{~mL}$ trifluoroethanol and $4.9 \mathrm{~mL} \mathrm{CH}_{2} \mathrm{Cl}_{2}$. The resin containing solution was filtered and dried in vacuo to yield the protected linear pentapeptide as a pale yellow solid (315 $\mathrm{mg}$, overall $84 \%)$.

\section{HO-Ala-Pro-Asp-Trp-Ser-NH 2 (Compound 8)}

The deprotected linear peptide was synthesized following the Side Chain Deprotection procedure using $0.47 \mathrm{~g}$ crude HO-Phe-Ala-Asp-Trp-Ser- $\mathrm{NH}_{2}$ (0.47 mmol, 1 equiv.), $1.9 \mathrm{~mL}$ of a mixed solution of TFA and $\mathrm{CH}_{2} \mathrm{Cl}_{2}(9: 1 \mathrm{v} / \mathrm{v})$ and $389 \mu \mathrm{L}$ anisole $(3.6 \mathrm{mmol}, 6$ equiv.) to generate the free side chains. The reaction mixture was stirred for 4 hours and monitored via LC/MS. Upon completion, the reaction mixture was worked up as described in the Side Chain Deprotection procedure and lyophilised to produce the crude linear peptide. The crude product was purified using HPLC to generate pure compound as a white solid $(38 \%)$.

LC/MS (ESI) $m / z$ : $[\mathrm{M}+\mathrm{H}]^{+}$calculated for $\mathrm{C}_{26} \mathrm{H}_{35} \mathrm{~N}_{6} \mathrm{O}_{9}{ }^{+}, 575.24$; found 575.30. The purity of the final compound was found to be $>95 \%$ by ${ }^{1} \mathrm{H}$ NMR, 2-D NMR (COSY, HSQC, HMBC) and LC/MS.

${ }^{1} \mathrm{H}$ NMR (600 MHz, D $\left.2 \mathrm{O}\right): \delta 7.63$ (d, J = $7.98 \mathrm{~Hz}, 1 \mathrm{H}$, ArH Trp), 7.51 (d, J = 8.19 Hz, 1H, ArH Trp), 7.34-7.08 (m, 3H, ArH Trp), 4.74 (t, J = 7.64 Hz, 1H, $\alpha \mathrm{H}$ Trp), 4.28-2.09 (m, 4H, $\alpha \mathrm{H}$ Ala, $\alpha \mathrm{H}$ Pro, $\alpha \mathrm{H}$ Asp, $\alpha \mathrm{H}$ Ser), 4.03-3.87 (m, 2H, $\beta \mathrm{H}$ Ser), 3.69-3.50 (m, 1H, $\delta \mathrm{H}$ Pro), 3.37-3.15 (m, 3H, $\delta \mathrm{H}$ Pro, $\beta \mathrm{H}$ Trp), 2.76-2.63 (m, 1H, $\beta \mathrm{H}$ Asp), 2.53-2.42 (m, 1H, $\beta \mathrm{H}$ Asp), 2.26-2.03 (m, 1H, $\gamma \mathrm{H}$ Pro), 2.00-1.78 (m, 3H, $\gamma \mathrm{H}$ Pro, $\beta \mathrm{H}$ Pro), 1.35 (d, J = 7.33 Hz, 3H, Ala). 


\section{Experimental Procedures for Compound 9}

\section{Resin-O-Phe- $\mathrm{NH}_{2}$}

The resin bound amino acid Resin-O-Phe- $\mathrm{NH}_{2}$ was synthesized according to the Resin Loading procedure. A sample of 2-ClTrt $\mathrm{Cl}$ resin $(0.6 \mathrm{~g}, 1.14 \mathrm{mmol} / \mathrm{g}$ loading, 1 equiv.) was placed in a reaction vessel and swelled with $\mathrm{CH}_{2} \mathrm{Cl}_{2}$ for 30 minutes then drained. To the swelled resin, $0.80 \mathrm{~g}$ Fmoc-Phe-OH (2.05 mmol, 3 equiv.) that was pre-dissolved in the minimum volume of DIPEA in $\mathrm{CH}_{2} \mathrm{Cl}_{2}(0.40 \mathrm{M})$ was added and the reaction was shaken at room temperature for $4 \mathrm{~h}$. The reaction mixture was drained and the resin was washed according to the Resin Loading protocol to produce Resin-O-Phe-NHFmoc. A sample of resin was removed and the resin loading was determined to be $0.493 \mathrm{mmol} / \mathrm{g}$. The Fmoc group was then removed following the Fmoc Removal procedure to produce Resin-O-Phe- $\mathrm{NH}_{2}$.

\section{Resin-O-Phe-Pro-NH}

Resin-O-Phe-Pro- $\mathrm{NH}_{2}$ was synthesized following the Coupling Reaction procedure, using $0.685 \mathrm{~g}$ ResinO-Phe- $\mathrm{NH}_{2}$ (0.34 mmol, 1 equiv.), 0.23 g Fmoc-Pro-OH (0.68 mmol, 2 equiv.), 1.13 mL HOAt (0.68 mmol, 2 equiv.), $0.21 \mathrm{~mL}$ DIC (1.35 mmol, 4 equiv.), and $0.91 \mathrm{~mL}$ of DMF to generate a concentration of $0.3 \mathrm{M}$. The coupling reaction was run for $2 \mathrm{~h}$ and a negative ninhydrin test was used to confirm reaction completion. The reaction mixture was drained to afford Resin-O-Phe-Pro-NHFmoc. The Fmoc group was then removed following the Fmoc Removal procedure to afford Resin-O-Phe-Pro- $\mathrm{NH}_{2}$.

\section{Resin-O-Phe-Pro-Asp(OtBu)- $\mathrm{NH}_{2}$}

Resin-O-Phe-Pro-Asp(OtBu)- $\mathrm{NH}_{2}$ was synthesized following the Coupling Reaction procedure, using Resin-O-Phe-Pro- $\mathrm{NH}_{2}$ from the previous reaction, $0.28 \mathrm{~g}$ Fmoc-Asp(OtBu)-OH (0.68 mmol, 2 equiv.), $1.13 \mathrm{~mL}$ HOAt (0.68 mmol, 2 equiv.), $0.21 \mathrm{~mL}$ DIC (1.35 mmol, 4 equiv.), and $0.91 \mathrm{~mL}$ of DMF to generate a concentration of $0.3 \mathrm{M}$. The coupling reaction was run for $2 \mathrm{~h}$ and a negative ninhydrin test was used to confirm reaction completion. The reaction mixture was drained to afford Resin-O-Phe-Pro- 
$\mathrm{Asp}(\mathrm{OtBu})-\mathrm{NHFmoc}$. The Fmoc group was then removed following the Fmoc Removal procedure to afford Resin-O-Phe-Pro-Asp(OtBu)- $\mathrm{NH}_{2}$.

\section{Resin-O-Phe-Pro-Asp(OtBu)-Trp(Boc)- $\mathrm{NH}_{2}$}

Resin-O-Phe-Pro_Asp(OtBu)-Trp(Boc)- $\mathrm{NH}_{2}$ was synthesized following the Coupling Reaction procedure, using Resin-O-Phe-Pro-Asp $(\mathrm{OtBu})-\mathrm{NH}_{2}$ from the previous reaction, $0.36 \mathrm{~g}$ Fmoc-Trp(Boc)$\mathrm{OH}$ (0.68 mmol, 2 equiv.), $0.091 \mathrm{~g} \mathrm{HOBt} \mathrm{(0.68} \mathrm{mmol,} 2$ equiv.), $0.21 \mathrm{~mL} \mathrm{DIC} \mathrm{(1.35} \mathrm{mmol,} 4$ equiv.), and $2.04 \mathrm{~mL}$ DMF to generate a concentration of $0.3 \mathrm{M}$. The coupling reaction was run for $2 \mathrm{~h}$ and a negative ninhydrin test was used to confirm reaction completion. The reaction mixture was drained to afford ResinO-Phe-Pro-Asp(OtBu)-Trp(Boc)-NHFmoc. The Fmoc group was then removed following the Fmoc Removal procedure to afford Resin-O-Phe-Pro-Asp(OtBu)-Trp(Boc)- $\mathrm{NH}_{2}$.

\section{Resin-O-Phe-Pro-Asp(OtBu)-Trp(Boc)-Asp(OtBu)-NH}

Resin-O-Phe-Pro-Asp(OtBu)-Trp(Boc)-Asp $(\mathrm{OtBu})-\mathrm{NH}_{2}$ was synthesized following the Coupling Reaction procedure, using Resin-O-Phe-Pro-Asp $(\mathrm{OtBu})-\mathrm{Trp}(\mathrm{Boc})-\mathrm{NH}_{2}$ from the previous reaction, 0.28 g Fmoc-Asp(OtBu)-OH (0.68 mmol, 2 equiv.), 1.13 mL HOAt (0.68 mmol, 2 equiv.), $0.21 \mathrm{~mL}$ DIC (1.35 mmol, 4 equiv.), and $0.91 \mathrm{~mL}$ DMF to generate a concentration of $0.3 \mathrm{M}$. The coupling reaction was run for $2 \mathrm{~h}$ and a negative ninhydrin test was used to confirm reaction completion. The reaction mixture was drained to afford Resin-O-Phe-Pro-Asp(OtBu)-Trp(Boc)-Asp(OtBu)-NHFmoc. The Fmoc group was then removed following the Fmoc Removal procedure to afford Resin-O-Phe-Pro-Asp(OtBu)-Trp(Boc)$\mathrm{Asp}(\mathrm{OtBu})-\mathrm{NH}_{2}$.

\section{HO-Phe-Pro-Asp(OtBu)-Trp(Boc)-Asp(OtBu)-NH}

The protected linear peptide HO-Phe-Pro-Asp(OtBu)-Trp(Boc)-Asp(OtBu)- $\mathrm{NH}_{2}$ was generated following the Resin Cleavage of Linear Peptide procedure. The linear peptide was cleaved from the resin 
using a solution of $3.3 \mathrm{~mL}$ trifluoroethanol and $3.3 \mathrm{~mL} \mathrm{CH}_{2} \mathrm{Cl}_{2}$. The resin containing solution was filtered and dried in vacuo to yield the protected linear peptide as a pale yellow solid (168.2 $\mathrm{mg}, 56 \%$ overall)

\section{HO-Phe-Pro-Asp-Trp-Asp-NH (Compound 9)}

The deprotected linear peptide was synthesized following the Side Chain Deprotection procedure using 0.11 g crude $\mathrm{HO}-\mathrm{Phe}-\mathrm{Pro}-\mathrm{Asp}(\mathrm{OtBu})-\mathrm{Trp}(\mathrm{Boc})-\mathrm{Asp}(\mathrm{OtBu})-\mathrm{NH}_{2}(0.13 \mathrm{mmol}, 1$ equiv. $), 0.56 \mathrm{~mL}$ of a mixed solution of TFA and $\mathrm{CH}_{2} \mathrm{Cl}_{2}(9: 1 \mathrm{v} / \mathrm{v})$ and $0.08 \mathrm{~mL}$ anisole $(0.75 \mathrm{mmol}, 6$ equiv. $)$ to remove side chain protecting groups. The reaction mixture was stirred for $6 \mathrm{~h}$ and monitored via LC/MS. Upon completion, the reaction mixture was worked up as described in the Side Chain Deprotection procedure to afford the crude linear peptide. The crude product was purified using HPLC, then lyophilized to generate pure compound as a white solid $(50.2 \mathrm{mg}, 33 \%$ overall). The purity of the final compound was found to be $>95 \%$ by ${ }^{1} \mathrm{H}$ NMR, 2-D NMR (COSY, HSQC, HMBC) and LC/MS.

LC/MS (ESI) m/z: $[\mathrm{M}+\mathrm{H}]^{+}$calculated for $\mathrm{C}_{33} \mathrm{H}_{38} \mathrm{~N}_{6} \mathrm{O}_{10}{ }^{+}$679.26, found 679.35 .

${ }^{1} \mathrm{H}$ NMR (600 MHz, $\left.\mathrm{D}_{2} \mathrm{O}\right): \delta 7.63$ (d, $J=7.92 \mathrm{~Hz}, 1 \mathrm{H}$, ArH Trp), 7.49 (d, $\left.J=8.16 \mathrm{~Hz}, 1 \mathrm{H}, \operatorname{ArH} \operatorname{Trp}\right)$, 7.38-7.23 (m, 7H, ArH Trp, ArH Phe), 7.15 (t, $J=7.50 \mathrm{~Hz}, 1 \mathrm{H}$, ArH Trp), 4.76-4.68 (m, 2H, $\alpha \mathrm{H}$ Asp, $\alpha \mathrm{H}$ Trp), $4.53(\mathrm{dd}, J=5.31 \mathrm{~Hz}, 1 \mathrm{H}, \alpha \mathrm{H}$ Phe), 4.28 (dd, $J=4.92 \mathrm{~Hz}, 1 \mathrm{H}, \alpha \mathrm{H}$ Asp), 4.05 (dd, $J=3.45 \mathrm{~Hz}$, 1H, $\alpha \mathrm{H}$ Pro), 3.48-3.44 (m, 1H, $\delta \mathrm{H}$ Pro), 3.24 (d, $J=7.80$ Hz, 2H, $\beta \mathrm{H}$ Trp), 3.22-2.97 (m, 3H, $\beta \mathrm{H}$ Phe, $\delta \mathrm{H}$ Pro), 2.91-2.50 (m, 4H, $\beta \mathrm{H}$ Asp, $\beta \mathrm{H}$ Asp), 1.98-1.92 (m, 1H, $\beta \mathrm{H}$ Pro), 1.72-1.63 (m, 2H, $\beta \mathrm{H}$ Pro, $\gamma \mathrm{H}$ Pro), 1.56-1.48 (m, 1H, $\gamma \mathrm{H}$ Pro).

\section{Experimental Procedures for Compound 10}

\section{Resin-O-D-Phe-NH}

The resin bound amino acid Resin-O-D-Phe- $\mathrm{NH}_{2}$ was synthesized according to the Resin Loading procedure. A sample of 2-ClTrt $\mathrm{Cl}$ resin $(0.5 \mathrm{~g}, 1.14 \mathrm{mmol} / \mathrm{g}$ loading, 1 equiv.) was placed in a reaction vessel and swelled with $\mathrm{CH}_{2} \mathrm{Cl}_{2}$ for 30 minutes then drained. To the resin, $0.66 \mathrm{~g}$ Fmoc-D-Phe-OH (1.7 
mmol, 3 equiv.) that was pre-dissolved in the minimum amount of DIPEA in $\mathrm{CH}_{2} \mathrm{Cl}_{2}(0.40 \mathrm{M})$ was added and the reaction was shaken at room temperature for 4 hours. The reaction mixture was drained and washed according to the protocol to produce Resin-O-D-Phe-NHFmoc. A sample of resin was removed and the resin loading was determined to be $0.445 \mathrm{mmol} / \mathrm{g}$. The Fmoc group was then removed following the Fmoc Removal procedure to produce Resin-O-D-Phe- $\mathrm{NH}_{2}$.

\section{Resin-O-D-Phe-D-Pro- $\mathrm{NH}_{2}$}

Resin-O-D-Phe-D-Pro- $\mathrm{NH}_{2}$ was synthesized following the Coupling Reaction procedure, using $0.5 \mathrm{~g}$ Resin-O-D-Phe-NH $\mathrm{NH}_{2}$ (0.22 mmol, 1 equiv.), 0.15 g Fmoc-D-Pro-OH (0.45 mmol, 2 equiv.), $0.74 \mathrm{~mL}$ HOAt (0.45 mmol, 2 equiv.), $0.14 \mathrm{~mL}$ DIC (0.89 mmol, 4 equiv.), and $0.60 \mathrm{~mL}$ of DMF to generate a concentration of $0.3 \mathrm{M}$. The coupling reaction was run for 4 hours and a negative ninhydrin test was used to confirm reaction completion. The reaction mixture was drained to afford Resin-O-D-Phe-D-ProNHFmoc. The Fmoc group was then removed following the Fmoc Removal procedure to afford ResinO-D-Phe-D-Pro- $\mathrm{NH}_{2}$.

\section{Resin-O-D-Phe-D-Pro-D-Asp(OtBu)-NH}

Resin-O-D-Phe-D-Pro-D-Asp $(\mathrm{OtBu})-\mathrm{NH}_{2}$ was synthesized following the Coupling Reaction procedure, using Resin-O-D-Phe-D-Pro- $\mathrm{NH}_{2}$ from the previous reaction, $0.18 \mathrm{~g}$ Fmoc-D-Asp(OtBu)-OH (0.45 mmol, 2 equiv.), $0.74 \mathrm{~mL}$ HOAt (0.45 mmol, 2 equiv.), $0.14 \mathrm{~mL}$ DIC (0.89 mmol, 4 equiv.), and $0.60 \mathrm{~mL}$ of DMF to generate a concentration of $0.3 \mathrm{M}$. The coupling reaction was run for 4 hours and a negative ninhydrin test was used to confirm reaction completion. The reaction mixture was drained to afford ResinO-D-Phe-D-Pro-D-Asp(OtBu)-NHFmoc. The Fmoc group was then removed following the Fmoc Removal procedure to afford Resin-O-D-Phe-D-Pro-D-Asp(OtBu)-NH $\mathrm{N}_{2}$.

\section{Resin-O-D-Phe-D-Pro-D-Asp(OtBu)-D-Trp(Boc)-NH}


Resin-O-D-Phe-D-Pro-D-Asp(OtBu)-D-Trp(Boc)- $\mathrm{NH}_{2}$ was synthesized following the Coupling Reaction procedure, using Resin-O-D-Phe-D-Pro-D-Asp $(\mathrm{OtBu})-\mathrm{NH}_{2}$ from the previous reaction, $0.23 \mathrm{~g}$ Fmoc-DTrp(Boc)-OH (0.45 mmol, 2 equiv.), 0.74 mL HOAt (0.45 mmol, 2 equiv.), 0.14 mL DIC (0.89 mmol, 4 equiv.), and $0.60 \mathrm{~mL}$ of DMF to generate a concentration of $0.3 \mathrm{M}$. The coupling reaction was run for 4 hours and a negative ninhydrin test was used to confirm reaction completion. The reaction mixture was drained to afford Resin-O-D-Phe-D-Pro-D-Asp(OtBu)-D-Trp(Boc)-NHFmoc. The Fmoc group was then removed following the Fmoc Removal procedure to afford Resin-O-D-Phe-D-Pro-D-Asp(OtBu)-D$\operatorname{Trp}($ Boc $)-\mathrm{NH}_{2}$.

\section{Resin-O-D-Phe-D-Pro-D-Asp(OtBu)-D-Trp(Boc)-D-Ser(OtBu)-NH}

Resin-O-D-Phe-D-Pro-D-Asp(OtBu)-D-Trp(Boc)-D-Ser(OtBu)- $\mathrm{NH}_{2}$ was synthesized following the Coupling Reaction procedure, using Resin-O-Phe-Pro-Asp(OtBu)-Trp(Boc)- $\mathrm{NH}_{2}$ from the previous reaction, 0.17 g Fmoc-D-Ser(OtBu)-OH (0.71 mmol, 2 equiv.), (0.45 mmol, 2 equiv.), $0.74 \mathrm{~mL}$ HOAt (0.45 mmol, 2 equiv.), $0.14 \mathrm{~mL}$ DIC (0.89 mmol, 4 equiv.), and $0.60 \mathrm{~mL}$ of DMF to generate a concentration of $0.3 \mathrm{M}$. The coupling reaction was run for 4 hours and a negative ninhydrin test was used to confirm reaction completion. The reaction mixture was drained to afford Resin-O-D-Phe-D-Pro-D$\mathrm{Asp}(\mathrm{OtBu})-\mathrm{Trp}(\mathrm{Boc})-\mathrm{D}-\mathrm{Ser}(\mathrm{OtBu})-\mathrm{NHFmoc}$. The Fmoc group was then removed following the Fmoc Removal procedure to afford Resin-O-D-Phe-D-Pro-D-Asp(OtBu)-D-Trp(Boc) -D-Ser(OtBu)-NH ${ }_{2}$.

\section{HO-D-Phe-D-Pro-D-Asp(OtBu)-D-Trp(Boc)-D-Ser(OtBu)-NH}

The protected linear pentapeptide HO-D-Phe-D-Pro-D-Asp(OtBu)-D-Trp(Boc) -D-Ser(OtBu)-NH $\mathrm{N}_{2}$ was generated following the Resin Cleavage of Linear Peptide procedure. The linear peptide was cleaved from the resin using a mixed solution of $3 \mathrm{~mL}$ trifluoroethanol and $3 \mathrm{~mL} \mathrm{CH}_{2} \mathrm{Cl}_{2}$. The resin containing solution was filtered and dried in vacuo to yield the protected linear pentapeptide as a pale yellow solid (183 $\mathrm{mg}$, overall $79 \%)$ 


\section{HO-D-Phe-D-Pro-D-Asp-D-Trp-D-Ser- $\mathrm{NH}_{2}$ (Compound 10)}

The deprotected linear peptide was synthesized following the Side Chain Deprotection procedure using 0.183 g crude HO-D-Phe-D-Pro-D-Asp-D-Trp-D-Ser-NH2 $(0.31 \mathrm{mmol}, 1$ equiv.), $0.73 \mathrm{~mL}$ of a mixed solution of TFA and $\mathrm{CH}_{2} \mathrm{Cl}_{2}(9: 1 \mathrm{v} / \mathrm{v})$ and $0.11 \mathrm{~mL}$ anisole $(1.05 \mathrm{mmol}, 6$ equiv.) to generate the free side chains. The reaction mixture was stirred for 4 hours and monitored via LC/MS. Upon completion, the reaction mixture was worked up as described in the Side Chain Deprotection procedure and lyophilised to produce the crude cyclic peptide. The crude product was purified using HPLC to generate pure compound as a white solid ( $2.8 \mathrm{mg}, 3 \%$ overall). The purity of the final compound was found to be $>95 \%$ by ${ }^{1} \mathrm{H}$ NMR, 2-D NMR (COSY, HSQC, HMBC) and LC/MS.

LC/MS (ESI) $m / z$ : $[\mathrm{M}+\mathrm{H}]^{+}$calculated for $\mathrm{C}_{32} \mathrm{H}_{39} \mathrm{~N}_{6} \mathrm{O}_{9}{ }^{+}, 651.28$; found 651.35 .

${ }^{1} \mathrm{H}$ NMR $\left(600 \mathrm{MHz}, \mathrm{D}_{2} \mathrm{O}\right): \delta 7.64(\mathrm{~d}, J=8.16 \mathrm{~Hz}, 1 \mathrm{H}, \operatorname{ArH} \operatorname{Trp}), 7.50(\mathrm{~d}, J=7.94 \mathrm{~Hz}, 1 \mathrm{H}, \operatorname{ArH} \operatorname{Trp})$, 7.41-7.22 (m, 7H, ArH Trp, ArH Phe), 7.20-7.12 (m, 1H ArH Trp), 4.76-4.71 (m, 1H, PheaCH), 4.514.43 (m, 1H, TrpaCH), 4.14-4.08 (m, 2H, Pro $\alpha C H$, Ser $\alpha C H), 4.01-3.91$ (m, 2H, SerßCH2), 3.52-3.45

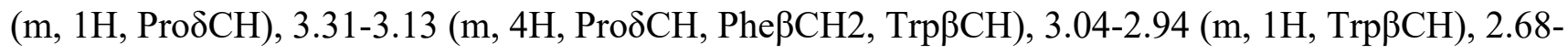

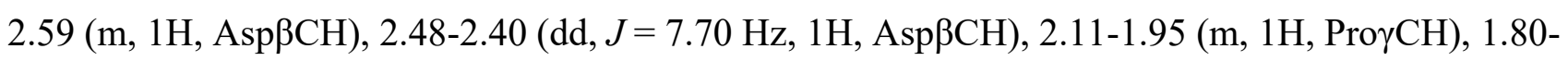

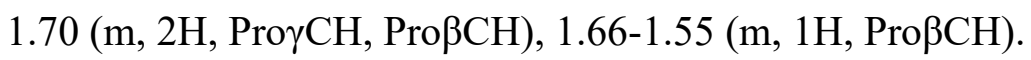

\section{Experimental Procedures for Compound 11}

\section{Resin-O-Arg(Pbf)-NH}

The resin bound amino acid $\mathrm{Resin}-\mathrm{O}-\mathrm{Arg}(\mathrm{Pbf})-\mathrm{NH}_{2}$ was synthesized according to the Resin Loading procedure. A sample of 2-ClTrt $\mathrm{Cl}$ resin $(0.6 \mathrm{~g}, 1.14 \mathrm{mmol} / \mathrm{g}$ loading, 1 equiv.) was placed in a reaction vessel and swelled with $\mathrm{CH}_{2} \mathrm{Cl}_{2}$ for 30 minutes then drained. To the swelled resin, $1.33 \mathrm{~g}$ Fmoc- $\mathrm{Arg}(\mathrm{Pbf})$ $\mathrm{OH}$ (2.05 mmol, 3 equiv.) that was predissolved in the minimum volume of DIPEA in $\mathrm{CH}_{2} \mathrm{Cl}_{2}(0.40 \mathrm{M})$ was added and the reaction was shaken at room temperature for $4 \mathrm{~h}$. The reaction mixture was drained 
and the resin was washed according to the Resin Loading protocol to produce Resin-O-Arg(Pbf)NHFmoc. A sample of resin was removed and the resin loading was determined to be $0.199 \mathrm{mmol} / \mathrm{g}$. The Fmoc group was then removed following the Fmoc Removal procedure to produce Resin-O-Arg(Pbf)$\mathrm{NH}_{2}$.

\section{Resin-O-Arg(Pbf)-Phe-NH}

Resin-O-Arg(Pbf)-Phe- $\mathrm{NH}_{2}$ was synthesized following the Coupling Reaction procedure, using $0.58 \mathrm{~g}$ Resin-O-Arg(Pbf)- $\mathrm{NH}_{2}$ (0.115 mmol, 1 equiv.), 0.089 g Fmoc-Phe-OH (0.23 mmol, 2 equiv.), $0.38 \mathrm{~mL}$ HOAt (0.23 mmol, 2 equiv.), $0.07 \mathrm{~mL}$ DIC (0.46 mmol, 4 equiv.), and $0.32 \mathrm{~mL}$ of DMF to generate a concentration of $0.3 \mathrm{M}$. The coupling reaction was run for $2 \mathrm{~h}$ and a negative ninhydrin test was used to confirm reaction completion. The reaction mixture was drained to afford Resin-O-Arg(Pbf)-PheNHFmoc. The Fmoc group was then removed following the Fmoc Removal procedure to afford Resin$\mathrm{O}-\mathrm{Arg}(\mathrm{Pbf})-\mathrm{Phe}-\mathrm{NH}_{2}$.

\section{Resin-O-Arg(Pbf)-Phe-Pro-NH}

Resin-O-Arg(Pbf)-Phe-Pro- $\mathrm{NH}_{2}$ was synthesized following the Coupling Reaction procedure, using Resin-O-Arg(Pbf)-Phe- $\mathrm{NH}_{2}$ from the previous reaction, $0.078 \mathrm{~g}$ Fmoc-Pro-OH (0.23 mmol, 2 equiv.), $0.38 \mathrm{~mL}$ HOAt (0.23 mmol, 2 equiv.), $0.07 \mathrm{~mL}$ DIC (0.46 mmol, 4 equiv.), and $0.32 \mathrm{~mL}$ of DMF to generate a concentration of $0.3 \mathrm{M}$. The coupling reaction was run for $2 \mathrm{~h}$ and a negative ninhydrin test was used to confirm reaction completion. The reaction mixture was drained to afford Resin-O-Arg(Pbf)Phe-Pro-NHFmoc. The Fmoc group was then removed following the Fmoc Removal procedure to afford Resin- Resin-O-Arg(Pbf)-Phe-Pro- $\mathrm{NH}_{2}$. 


\section{Resin-O-Arg(Pbf)-Phe-Pro-Asp(OtBu)--NH2}

Resin-O-Arg(Pbf)-Phe-Pro-Asp $(\mathrm{OtBu})-\mathrm{NH}_{2}$ was synthesized following the Coupling Reaction procedure, using Resin-O-Arg(Pbf)-Phe-Pro- $\mathrm{NH}_{2}$ from the previous reaction, $0.095 \mathrm{~g}$ Fmoc-Asp(OtBu)OH (0.23 mmol, 2 equiv.), $0.38 \mathrm{~mL}$ HOAt (0.23 mmol, 2 equiv.), $0.07 \mathrm{~mL}$ DIC (0.46 mmol, 4 equiv.), and $0.32 \mathrm{~mL}$ DMF to generate a concentration of $0.3 \mathrm{M}$. The coupling reaction was run for $2 \mathrm{~h}$ and a negative ninhydrin test was used to confirm reaction completion. The reaction mixture was drained to afford Resin-O-Arg(Pbf)-Phe-Pro-Asp(OtBu)-NHFmoc. The Fmoc group was then removed following the Fmoc Removal procedure to afford Resin-O-Arg(Pbf)-Phe-Pro-Asp(OtBu)- $\mathrm{NH}_{2}$.

\section{Resin-O-Arg(Pbf)-Phe-Pro-Asp(OtBu)-Trp(Boc)-NH}

Resin-O-Arg(Pbf)-Phe-Pro-Asp(OtBu)-Trp(Boc)- $\mathrm{NH}_{2}$ was synthesized following the Coupling Reaction procedure, using Resin-O-Arg(Pbf)-Phe-Pro-Asp $(\mathrm{OtBu})-\mathrm{NH}_{2}$ from the previous reaction, $0.12 \mathrm{~g}$ FmocTrp(Boc)-OH (0.23 mmol, 2 equiv.), 0.031 g HOBt (0.23 mmol, 2 equiv.), 0.07 mL DIC (0.46 mmol, 4 equiv.), and $0.7 \mathrm{~mL}$ DMF to generate a concentration of $0.3 \mathrm{M}$. The coupling reaction was run for $2 \mathrm{~h}$ and a negative ninhydrin test was used to confirm reaction completion. The reaction mixture was drained to afford Resin-O-Arg(Pbf)-Phe-Pro-Asp(OtBu)-Trp(Boc)-NHFmoc. The Fmoc group was then removed following the Fmoc Removal procedure to afford Resin-O-Arg(Pbf)-Phe-Pro-Asp(OtBu)-Trp(Boc)-NH . $_{2}$

\section{Resin-O-Arg(Pbf)-Phe-Pro-Asp(OtBu)-Trp(Boc)-Ser(Trt)-NH}

Resin-O-Arg(Pbf)-Phe-Pro-Asp(OtBu)-Trp(Boc)-Ser(Trt)- $\mathrm{NH}_{2}$ was synthesized following the Coupling Reaction procedure, using Resin-O-Arg( $(\mathrm{Pbf})-\mathrm{Phe}-\mathrm{Pro}-\mathrm{Asp}(\mathrm{OtBu})-\mathrm{Trp}(\mathrm{Boc})-\mathrm{NH}_{2}$ from the previous reaction, 0.13 g Fmoc-Ser(Trt)-OH (0.23 mmol, 2 equiv.), 0.031 g HOBt (0.23 mmol, 2 equiv.), $0.07 \mathrm{~mL}$ DIC (0.46 mmol, 4 equiv.), and $0.7 \mathrm{~mL}$ DMF to generate a concentration of $0.3 \mathrm{M}$. The coupling reaction was run for $2 \mathrm{~h}$ and a negative ninhydrin test was used to confirm reaction completion. The reaction mixture was drained to afford Resin-O-Arg(Pbf)-Phe-Pro-Asp(OtBu)-Trp(Boc)-Ser(Trt)-NHFmoc. The 
Fmoc group was then removed following the Fmoc Removal procedure to afford Resin-O-Arg(Pbf)-PhePro-Asp(OtBu)-Trp(Boc)-Ser(Trt)- $\mathrm{NH}_{2}$.

\section{HO-Arg(Pbf)-Phe-Pro-Asp(OtBu)-Trp(Boc)-Ser(Trt)-NH}

The protected linear peptide HO-Arg(Pbf)-Phe-Pro-Asp(OtBu)-Trp(Boc)-Ser(Trt)- $\mathrm{NH}_{2}$ was generated following the Resin Cleavage of Linear Peptide procedure. The linear peptide was cleaved from the resin using a solution of $3 \mathrm{~mL}$ trifluoroethanol and $3 \mathrm{~mL} \mathrm{CH}_{2} \mathrm{Cl}_{2}$. The resin containing solution was filtered and dried in vacuo to yield the protected linear peptide as a pale yellow solid (130 $\mathrm{mg}, 77 \%$ overall).

\section{HO-Arg-Phe-Pro-Asp-Trp-Ser-NH 2 (Compound 11)}

The deprotected linear peptide was synthesized following the Side Chain Deprotection procedure using 0.12 g crude HO-Arg(Pbf)-Phe-Pro-Asp(OtBu)-Trp(Boc)-Ser(Trt)-NH $\mathrm{NH}_{2}(0.082 \mathrm{mmol}, 1$ equiv.), $0.60 \mathrm{~mL}$ of a mixed solution of TFA and $\mathrm{CH}_{2} \mathrm{Cl}_{2}(9: 1 \mathrm{v} / \mathrm{v})$ and $0.07 \mathrm{~mL}$ anisole $(0.66 \mathrm{mmol}, 8$ equiv.) to remove side chain protecting groups. The reaction mixture was stirred for $12 \mathrm{~h}$ and Monitored via LC/MS. Upon completion, the reaction mixture was worked up as described in the Side Chain Deprotection procedure to afford the crude linear peptide. The crude product was purified using HPLC, then lyophilized to generate pure compound as a white solid $(29.8 \mathrm{mg}, 35 \%$ overall). The purity of the final compound was found to be $>95 \%$ by ${ }^{1} \mathrm{H}$ NMR, 2-D NMR (COSY, HSQC, HMBC) and LC/MS.

LC/MS (ESI) $m / z:[\mathrm{M}+\mathrm{H}]^{+}$calculated for $\mathrm{C}_{38} \mathrm{H}_{50} \mathrm{~N}_{10} \mathrm{O}_{10}{ }^{+}$807.37, found 807.35.

${ }^{1} \mathrm{H}$ NMR (600 MHz, $\left.\mathrm{D}_{2} \mathrm{O}\right): \delta 7.63(\mathrm{~d}, J=7.92 \mathrm{~Hz}, 1 \mathrm{H}, \operatorname{ArH} \operatorname{Trp}), 7.49(\mathrm{~d}, J=8.16 \mathrm{~Hz}, 1 \mathrm{H}, \operatorname{ArH} \operatorname{Trp})$, 7.39-7.23 (m, 7H, ArH Trp, ArH Phe), 7.16 (t, $J=7.50$ Hz, 1H, ArH Trp), 4.75-4.71 (m, 2H, $\alpha \mathrm{H}$ Asp, $\alpha \mathrm{H}$ Phe), 4.60 (dd, $J=5.52 \mathrm{~Hz}, 1 \mathrm{H}, \alpha \mathrm{H}$ Trp), 4.16-4.13 (m, 2H, $\alpha \mathrm{H}$ Arg, $\alpha \mathrm{H}$ Ser), 4.05-3.92 (m, 3H, $\alpha \mathrm{H}$ Pro, $\beta$ H Ser), 3.53-3.50 (m, 1H, $\delta$ H Pro), 3.28-3.13 (m, 6H, $\beta \mathrm{H}$ Trp, $\beta \mathrm{H}$ Phe, $\delta \mathrm{H}$ Arg, $\delta \mathrm{H}$ Pro), 2.99 (dd, $J=10.11 \mathrm{~Hz}, 1 \mathrm{H}, \beta \mathrm{H}$ Phe), 2.66-2.42 (m, 2H, $\beta \mathrm{H}$ Asp), 2.03-1.96 (m, 1H, $\beta \mathrm{H}$ Pro), 1.85-1.79 (m, $1 \mathrm{H}, \beta \mathrm{H}$ Arg), 1.72-1.65 (m, 2H, $\beta \mathrm{H}$ Arg, $\gamma \mathrm{H}$ Pro), 1.57-1.45 (m, 4H, $\gamma \mathrm{H}$ Arg, $\beta \mathrm{H}$ Pro, $\gamma \mathrm{H}$ Pro). 


\section{Experimental Procedures for Compound 12}

\section{Resin-O-Asp(OtBu)- $\mathrm{NH}_{2}$}

The resin bound amino acid Resin-O-Asp $(\mathrm{OtBu})-\mathrm{NH}_{2}$ was synthesized according to the Resin Loading procedure. A sample of 2-ClTrt $\mathrm{Cl}$ resin $(0.6 \mathrm{~g}, 1.14 \mathrm{mmol} / \mathrm{g}$ loading, 1 equiv.) was placed in a reaction vessel and swelled with $\mathrm{CH}_{2} \mathrm{Cl}_{2}$ for 30 minutes then drained. To the swelled resin, $0.84 \mathrm{~g}$ Fmoc$\mathrm{Asp}(\mathrm{OtBu})-\mathrm{OH}\left(2.05 \mathrm{mmol}, 3\right.$ equiv.) that was predissolved in the minimum volume of DIPEA in $\mathrm{CH}_{2} \mathrm{Cl}_{2}$ $(0.40 \mathrm{M})$ was added and the reaction was shaken at room temperature for $4 \mathrm{~h}$. The reaction mixture was drained and the resin was washed according to the Resin Loading protocol to produce Resin-O$\mathrm{Asp}(\mathrm{OtBu})$-NHFmoc. A sample of resin was removed and the resin loading was determined to be 0.427 $\mathrm{mmol} / \mathrm{g}$. The Fmoc group was then removed following the Fmoc Removal procedure to produce Resin$\mathrm{O}-\mathrm{Asp}(\mathrm{OtBu})-\mathrm{NH}_{2}$.

\section{Resin-O-Asp(OtBu)-Arg(Pbf)-NH}

Resin-O-Asp $(\mathrm{OtBu})-\mathrm{Arg}(\mathrm{Pbf})-\mathrm{NH}_{2}$ was synthesized following the Coupling Reaction procedure, using 0.7 g Resin-O-Asp(OtBu)- $\mathrm{NH}_{2}$ (0.3 mmol, 1 equiv.), 0.39 g Fmoc-Arg(Pbf)-OH (0.60 mmol, 2 equiv.), $1.0 \mathrm{~mL}$ HOAt (0.60 mmol, 2 equiv.), $0.19 \mathrm{~mL}$ DIC (1.20 mmol, 4 equiv.), and $0.80 \mathrm{~mL}$ of DMF to generate a concentration of $0.3 \mathrm{M}$. The coupling reaction was run for $2 \mathrm{~h}$ and a negative ninhydrin test was used to confirm reaction completion. The reaction mixture was drained to afford Resin-O$\mathrm{Asp}(\mathrm{OtBu})-\operatorname{Arg}(\mathrm{Pbf})-\mathrm{NHFmoc}$. The Fmoc group was then removed following the Fmoc Removal procedure to afford Resin-O-Asp(OtBu)-Arg( $\mathrm{Pbf})-\mathrm{NH}_{2}$.

\section{Resin-O-Asp(OtBu)-Arg(Pbf)-Phe--NH}

Resin-O-Asp(OtBu)-Arg( $\mathrm{Pbf})-\mathrm{Phe}-\mathrm{NH}_{2}$ was synthesized following the Coupling Reaction procedure, using Resin-O-Asp(OtBu)-Arg(Pbf)- $\mathrm{NH}_{2}$ from the previous reaction, $0.23 \mathrm{~g}$ Fmoc-Phe-OH (0.60 mmol, 
2 equiv.), $1.0 \mathrm{~mL}$ HOAt (0.60 mmol, 2 equiv.), $0.19 \mathrm{~mL}$ DIC (1.20 mmol, 4 equiv.), and $0.8 \mathrm{~mL}$ of DMF to generate a concentration of $0.3 \mathrm{M}$. The coupling reaction was run for $2 \mathrm{~h}$ and a negative ninhydrin test was used to confirm reaction completion. The reaction mixture was drained to afford Resin-OAsp(OtBu)-Arg(Pbf)-Phe-NHFmoc. The Fmoc group was then removed following the Fmoc Removal procedure to afford Resin-O-Asp(OtBu)-Arg( $\mathrm{Pbf})-\mathrm{Phe}-\mathrm{NH}_{2}$.

\section{Resin-O-Asp(OtBu)-Arg(Pbf)-Phe-Pro--NH2}

Resin-O-Asp(OtBu)-Arg(Pbf)-Phe-Pro- $\mathrm{NH}_{2}$ was synthesized following the Coupling Reaction procedure, using Resin-O-Asp $(\mathrm{OtBu})-\mathrm{Arg}(\mathrm{Pbf})-\mathrm{Phe}-\mathrm{NH}_{2}$ from the previous reaction, $0.20 \mathrm{~g}$ Fmoc-Pro$\mathrm{OH}$ (0.60 mmol, 2 equiv.), $1.0 \mathrm{~mL}$ HOAt (0.60 mmol, 2 equiv.), $0.19 \mathrm{~mL}$ DIC (1.2 mmol, 4 equiv.), and $0.80 \mathrm{~mL}$ of DMF to generate a concentration of $0.3 \mathrm{M}$. The coupling reaction was run for $2 \mathrm{~h}$ and a negative ninhydrin test was used to confirm reaction completion. The reaction mixture was drained to afford Resin-O-Asp(OtBu)-Arg(Pbf)-Phe-Pro-NHFmoc. The Fmoc group was then removed following the Fmoc Removal procedure to afford Resin-O-Asp(OtBu)-Arg(Pbf)-Phe-Pro- $\mathrm{NH}_{2}$.

\section{Resin-O-Asp(OtBu)-Arg(Pbf)-Phe-Pro-Asp(OtBu)-NH2}

Resin-O-Asp(OtBu)-Arg(Pbf)-Phe-Pro-Asp(OtBu)- $\mathrm{NH}_{2}$ was synthesized following the Coupling Reaction procedure, using Resin-O-Asp(OtBu)-Arg( $\mathrm{Pbf})-\mathrm{Phe}-\mathrm{Pro}-\mathrm{NH}_{2}$ from the previous reaction, 0.25 g Fmoc-Asp(OtBu)-OH (0.60 mmol, 2 equiv.), 1.0 mL HOAt (0.6 mmol, 2 equiv.), 0.19 mL DIC (1.20 mmol, 4 equiv.), and $0.8 \mathrm{~mL}$ DMF to generate a concentration of $0.3 \mathrm{M}$. The coupling reaction was run for $2 \mathrm{~h}$ and a negative ninhydrin test was used to confirm reaction completion. The reaction mixture was drained to afford Resin-O-Arg(Pbf)-Phe-Pro-Asp(OtBu)-NHFmoc. The Fmoc group was then removed following the Fmoc Removal procedure to afford Resin-O-Arg(Pbf)-Phe-Pro-Asp(OtBu)- $\mathrm{NH}_{2}$. 


\section{Resin-O-Asp(OtBu)-Arg(Pbf)-Phe-Pro-Asp(OtBu)-Trp(Boc)-NH}

Resin-O-Asp(OtBu)-Arg(Pbf)-Phe-Pro-Asp(OtBu)-Trp(Boc)- $\mathrm{NH}_{2}$ was synthesized following the Coupling Reaction procedure, using Resin-O-Arg( $\mathrm{Pbf})-\mathrm{Phe}-\mathrm{Pro}-\mathrm{Asp}(\mathrm{OtBu})-\mathrm{NH}_{2}$ from the previous reaction, 0.31 g Fmoc-Trp(Boc)-OH (0.60 mmol, 2 equiv.), 0.08 g HOBt (0.6 mmol, 2 equiv.), $0.19 \mathrm{~mL}$ DIC (1.20 mmol, 4 equiv.), and $1.80 \mathrm{~mL}$ DMF to generate a concentration of $0.3 \mathrm{M}$. The coupling reaction was run for $2 \mathrm{~h}$ and a negative ninhydrin test was used to confirm reaction completion. The reaction mixture was drained to afford Resin-O-Asp(OtBu)-Arg(Pbf)-Phe-Pro-Asp(OtBu)-Trp(Boc)-NHFmoc. The Fmoc group was then removed following the Fmoc Removal procedure to afford Resin-O$\operatorname{Asp}(\mathrm{OtBu})-\mathrm{Arg}(\mathrm{Pbf})-\mathrm{Phe}-\mathrm{Pro}-\mathrm{Asp}(\mathrm{OtBu})-\mathrm{Trp}(\mathrm{Boc})-\mathrm{NH}_{2}$.

\section{Resin-O-Asp(OtBu)-Arg(Pbf)-Phe-Pro-Asp(OtBu)-Trp(Boc)-Ser(Trt)-NH}

Resin-O-Asp(OtBu)-Arg(Pbf)-Phe-Pro-Asp(OtBu)-Trp(Boc)-Ser(Trt)-NH $\mathrm{NH}_{2}$ was synthesized following the Coupling Reaction procedure, using Resin-O-Asp(OtBu)-Arg(Pbf)-Phe-Pro-Asp(OtBu)-Trp(Boc)$\mathrm{NH}_{2}$ from the previous reaction, $0.34 \mathrm{~g}$ Fmoc-Ser(Trt)-OH (0.60 mmol, 2 equiv.), $0.08 \mathrm{~g} \mathrm{HOBt}(0.60$ mmol, 2 equiv.), $0.19 \mathrm{~mL}$ DIC (1.20 mmol, 4 equiv.), and $1.8 \mathrm{~mL}$ DMF to generate a concentration of $0.3 \mathrm{M}$. The coupling reaction was run for $2 \mathrm{~h}$ and a negative ninhydrin test was used to confirm reaction completion. The reaction mixture was drained to afford Resin-O-Asp(OtBu)-Arg(Pbf)-Phe-ProAsp(OtBu)-Trp(Boc)-Ser(Trt)-NHFmoc. The Fmoc group was then removed following the Fmoc Removal procedure to afford Resin-O-Asp(OtBu)-Arg(Pbf)-Phe-Pro-Asp(OtBu)-Trp(Boc)-Ser(Trt)$\mathrm{NH}_{2}$.

\section{HO-Asp(OtBu)-Arg(Pbf)-Phe-Pro-Asp(OtBu)-Trp(Boc)-Ser(Trt)-NH}

The protected linear peptide $\mathrm{HO}-\mathrm{Asp}(\mathrm{OtBu})-\mathrm{Arg}(\mathrm{Pbf})-\mathrm{Phe}-\mathrm{Pro}-\mathrm{Asp}(\mathrm{OtBu})-\mathrm{Trp}(\mathrm{Boc})-\mathrm{Ser}(\mathrm{Trt})-\mathrm{NH}_{2}$ was generated following the Resin Cleavage of Linear Peptide procedure. The linear peptide was cleaved from the resin using a solution of $4 \mathrm{~mL}$ trifluoroethanol and $4 \mathrm{~mL} \mathrm{CH} \mathrm{Cl}_{2}$. The resin containing solution was 
filtered and dried in vacuo to yield the protected linear peptide as a pale yellow solid (325 $\mathrm{mg}, 67 \%$ overall).

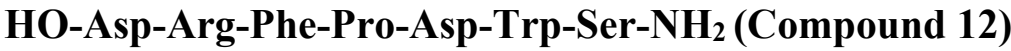

The deprotected linear peptide was synthesized following the Side Chain Deprotection procedure using 0.13 g crude HO-Asp(OtBu)-Arg(Pbf)-Phe-Pro-Asp(OtBu)-Trp(Boc)-Ser(Trt)- $\mathrm{NH}_{2}$ (0.08 mmol, 1 equiv.), $0.65 \mathrm{~mL}$ of a mixed solution of TFA and $\mathrm{CH}_{2} \mathrm{Cl}_{2}(9: 1 \mathrm{v} / \mathrm{v})$ and $0.09 \mathrm{~mL}$ anisole $(0.80 \mathrm{mmol}, 10$ equiv.) to remove side chain protecting groups. The reaction mixture was stirred for $12 \mathrm{~h}$ and monitored via LC/MS. Upon completion, the reaction mixture was worked up as described in the Side Chain Deprotection procedure to afford the crude linear peptide. The crude product was purified using HPLC, then lyophilized to generate pure compound as a white solid (18.1 mg, 16\% overall). The purity of the final compound was found to be $>95 \%$ by ${ }^{1} \mathrm{H}$ NMR, 2-D NMR (COSY, HSQC, HMBC) and LC/MS. LC/MS (ESI) $m / z:[\mathrm{M}+\mathrm{H}]^{+}$calculated for $\mathrm{C}_{42} \mathrm{H}_{55} \mathrm{~N}_{11} \mathrm{O}_{13}{ }^{+}$922.40, found 922.35 .

${ }^{1} \mathrm{H}$ NMR (600 MHz, D $\left.2 \mathrm{O}\right): \delta 7.63$ (d, $J=7.98$ Hz, 1H, ArH Trp), 7.49 (d, J=8.1 Hz, 1H, ArH Trp), 7.397.23 (m, 7H, ArH Trp, ArH Phe), 7.15 (t, J=7.5 Hz, 1H, ArH Trp), 4.76-4.70 (m, 2H, $\alpha \mathrm{H}$ Asp, $\alpha \mathrm{H}$ Trp), 4.57 (dd, $J=6.03 \mathrm{~Hz}, 1 \mathrm{H}, \alpha \mathrm{H}$ Phe), 4.49 (dd, $J=5.16 \mathrm{~Hz}, 1 \mathrm{H}, \alpha \mathrm{H}$ Asp), 4.34-4.31 (m, 1H, $\alpha \mathrm{H} \mathrm{Arg),} 4.15$ (t, $J=4.98$ Hz, 1H, $\alpha \mathrm{H}$ Ser), 4.03-3.93 (m, 3H, $\alpha \mathrm{H}$ Pro, $\beta \mathrm{H}$ Ser), 3.57-3.53 (m, 1H, $\delta \mathrm{H}$ Pro), 3.25 (d, $J=$ 7.86 Hz, 2H, $\beta \mathrm{H}$ Trp), 3.22-3.16 (m, 4H, $\beta \mathrm{H}$ Phe, $\delta \mathrm{H}$ Pro, $\delta \mathrm{H}$ Arg), 3.01 (dd, $J=10.02 \mathrm{~Hz}, 1 \mathrm{H}, \beta \mathrm{H}$ Phe), 2.81-2.43 (m, 4H, $\beta \mathrm{H}$ Asp), 2.07-1.99 (m, 1H, $\beta \mathrm{H}$ Pro), 1.87-1.81 (m, 1H, $\beta \mathrm{H} \mathrm{Arg),} \mathrm{1.74-1.64} \mathrm{(m,} \mathrm{2H,}$ $\gamma \mathrm{H}$ Pro, $\beta \mathrm{H}$ Arg), 1.62-1.48 (m, 4H, $\gamma \mathrm{H}$ Arg, $\beta \mathrm{H}$ Pro, $\gamma \mathrm{H}$ Pro).

\section{Experimental Procedures for Compound 13}

\section{Resin-O-Trp(Boc)-NH}

The resin bound amino acid Resin-O-Trp(Boc)- $\mathrm{NH}_{2}$ was synthesized according to the Resin Loading procedure. A sample of 2-ClTrt $\mathrm{Cl}$ resin $(0.6 \mathrm{~g}, 1.14 \mathrm{mmol} / \mathrm{g}$ loading, 1 equiv. $)$ was placed in a reaction 
vessel and swelled with $\mathrm{CH}_{2} \mathrm{Cl}_{2}$ for 30 minutes then drained. To the swelled resin, $1.08 \mathrm{~g}$ Fmoc-Trp(Boc)$\mathrm{OH}$ (2.05 mmol, 3 equiv.) that was predissolved in the minimum volume of DIPEA in $\mathrm{CH}_{2} \mathrm{Cl}_{2}(0.40 \mathrm{M})$ was added and the reaction was shaken at room temperature for $4 \mathrm{~h}$. The reaction mixture was drained and the resin was washed according to the Resin Loading protocol to produce Resin-O-Trp(Boc)NHFmoc. A sample of resin was removed and the resin loading was determined to be $0.322 \mathrm{mmol} / \mathrm{g}$. The Fmoc group was then removed following the Fmoc Removal procedure to produce Resin-O-Trp(Boc)$\mathrm{NH}_{2}$.

\section{Resin-O-Trp(Boc)-Asp(OtBu)-NH}

Resin-O-Trp(Boc)-Asp(OtBu)- $\mathrm{NH}_{2}$ was synthesized following the Coupling Reaction procedure, using 0.67 g Resin-O-Trp(Boc)-NH 2 (0.21 mmol, 1 equiv.), 0.18 g Fmoc-Asp(OtBu)-OH (0.43 mmol, 2 equiv.), $0.71 \mathrm{~mL}$ HOAt (0.43 mmol, 2 equiv.), $0.13 \mathrm{~mL}$ DIC (0.86 mmol, 4 equiv.), and $0.60 \mathrm{~mL}$ of DMF to generate a concentration of $0.3 \mathrm{M}$. The coupling reaction was run for $2 \mathrm{~h}$ and a negative ninhydrin test was used to confirm reaction completion. The reaction mixture was drained to afford Resin-O-Trp(Boc)Asp(OtBu)-NHFmoc. The Fmoc group was then removed following the Fmoc Removal procedure to afford Resin-O-Trp(Boc)-Asp(OtBu)- $\mathrm{NH}_{2}$.

\section{Resin-O-Trp(Boc)-Asp(OtBu)-Arg(Pbf)-NH}

Resin-O-Trp(Boc)-Asp(OtBu)-Arg(Pbf)- $\mathrm{NH}_{2}$ was synthesized following the Coupling Reaction procedure, using Resin-O-Trp(Boc)-Asp(OtBu)- $\mathrm{NH}_{2}$ from the previous reaction, $0.28 \mathrm{~g}$ Fmoc-Arg(Pbf)$\mathrm{OH}$ (0.43 mmol, 2 equiv.), $0.71 \mathrm{~mL}$ HOAt (0.43 mmol, 2 equiv.), $0.13 \mathrm{~mL}$ DIC (0.86 mmol, 4 equiv.), and $0.60 \mathrm{~mL}$ of DMF to generate a concentration of $0.3 \mathrm{M}$. The coupling reaction was run for $2 \mathrm{~h}$ and a negative ninhydrin test was used to confirm reaction completion. The reaction mixture was drained to afford Resin-O-Trp(Boc)-Asp(OtBu)-Arg(Pbf)-NHFmoc. The Fmoc group was then removed following the Fmoc Removal procedure to afford Resin-O-Trp(Boc)-Asp(OtBu)-Arg(Pbf)- $\mathrm{NH}_{2}$. 


\section{Resin-O-Trp(Boc)-Asp(OtBu)-Arg(Pbf)-Phe- $\mathrm{NH}_{2}$}

Resin-O-Trp(Boc)-Asp(OtBu)-Arg(Pbf)-Phe- $\mathrm{NH}_{2}$ was synthesized following the Coupling Reaction procedure, using Resin-O-Trp(Boc)-Asp(OtBu)-Arg( $\mathrm{Pbf})-\mathrm{NH}_{2}$ from the previous reaction, $0.17 \mathrm{~g}$ FmocPhe-OH (0.43 mmol, 2 equiv.), $0.71 \mathrm{~mL}$ HOAt (0.43 mmol, 2 equiv.), $0.13 \mathrm{~mL}$ DIC (0.86 mmol, 4 equiv.), and $0.6 \mathrm{~mL}$ of DMF to generate a concentration of $0.3 \mathrm{M}$. The coupling reaction was run for $2 \mathrm{~h}$ and a negative ninhydrin test was used to confirm reaction completion. The reaction mixture was drained to afford Resin-O-Trp(Boc)-Asp(OtBu)-Arg(Pbf)-Phe-NHFmoc. The Fmoc group was then removed following the Fmoc Removal procedure to afford Resin-O-Trp(Boc)-Asp(OtBu)-Arg(Pbf)-Phe- $\mathrm{NH}_{2}$.

\section{Resin-O-Trp(Boc)-Asp(OtBu)-Arg(Pbf)-Phe-Pro-NH}

Resin-O-Trp(Boc)-Asp(OtBu)-Arg(Pbf)-Phe-Pro- $\mathrm{NH}_{2}$ was synthesized following the Coupling Reaction procedure, using Resin-O-Trp(Boc)-Asp(OtBu)-Arg( $\mathrm{Pbf})-\mathrm{Phe}-\mathrm{NH}_{2}$ from the previous reaction, $0.14 \mathrm{~g}$ Fmoc-Pro-OH (0.43 mmol, 2 equiv.), $0.71 \mathrm{~mL}$ HOAt (0.43 mmol, 2 equiv.), $0.13 \mathrm{~mL}$ DIC (0.86 mmol, 4 equiv.), and $0.60 \mathrm{~mL}$ of DMF to generate a concentration of $0.3 \mathrm{M}$. The coupling reaction was run for $2 \mathrm{~h}$ and a negative ninhydrin test was used to confirm reaction completion. The reaction mixture was drained to afford Resin-O-Trp(Boc)-Asp(OtBu)-Arg(Pbf)-Phe-Pro-NHFmoc. The Fmoc group was then removed following the Fmoc Removal procedure to afford Resin-O-Trp(Boc)-Asp(OtBu)-Arg(Pbf)-PhePro- $\mathrm{NH}_{2}$.

\section{Resin-O-Trp(Boc)-Asp(OtBu)-Arg(Pbf)-Phe-Pro-Asp(OtBu)-NH}

Resin-O-Trp(Boc)-Asp(OtBu)-Arg(Pbf)-Phe-Pro-Asp $(\mathrm{OtBu})-\mathrm{NH}_{2}$ was synthesized following the Coupling Reaction procedure, using Resin-O-Trp(Boc)-Asp(OtBu)-Arg(Pbf)-Phe-Pro- $\mathrm{NH}_{2}$ from the previous reaction, 0.18 g Fmoc-Asp(OtBu)-OH (0.43 mmol, 2 equiv.), $0.71 \mathrm{~mL}$ HOAt (0.43 mmol, 2 equiv.), $0.13 \mathrm{~mL}$ DIC (0.86 mmol, 4 equiv.), and $0.60 \mathrm{~mL}$ DMF to generate a concentration of $0.3 \mathrm{M}$. The coupling reaction was run for $2 \mathrm{~h}$ and a negative ninhydrin test was used to confirm reaction 
completion. The reaction mixture was drained to afford Resin-O-Trp(Boc)-Asp(OtBu)-Arg(Pbf)-PhePro-Asp(OtBu)-NHFmoc. The Fmoc group was then removed following the Fmoc Removal procedure to afford Resin-O-Trp(Boc)-Asp(OtBu)-Arg(Pbf)-Phe-Pro-Asp(OtBu)- $\mathrm{NH}_{2}$.

\section{Resin-O-Trp(Boc)-Asp(OtBu)-Arg(Pbf)-Phe-Pro-Asp(OtBu)-Trp(Boc)-NH}

Resin-O-Trp(Boc)-Asp(OtBu)-Arg(Pbf)-Phe-Pro-Asp(OtBu)-Trp(Boc)- $\mathrm{NH}_{2}$ was synthesized following the Coupling Reaction procedure, using afford Resin-O-Trp(Boc)-Asp(OtBu)-Arg(Pbf)-Phe-Pro$\mathrm{Asp}(\mathrm{OtBu})-\mathrm{NH}_{2}$ from the previous reaction, $0.23 \mathrm{~g}$ Fmoc-Trp(Boc)-OH (0.43 mmol, 2 equiv.), $0.058 \mathrm{~g}$ HOBt (0.43 mmol, 2 equiv.), $0.13 \mathrm{~mL}$ DIC (0.86 mmol, 4 equiv.), and $1.3 \mathrm{~mL}$ DMF to generate a concentration of $0.3 \mathrm{M}$. The coupling reaction was run for $2 \mathrm{~h}$ and a negative ninhydrin test was used to confirm reaction completion. The reaction mixture was drained to afford Resin-O-Trp(Boc)-Asp(OtBu)$\operatorname{Arg}(\mathrm{Pbf})$-Phe-Pro-Asp(OtBu)-Trp(Boc)-NHFmoc. The Fmoc group was then removed following the Fmoc Removal procedure to afford Resin-O-Trp(Boc)-Asp(OtBu)-Arg(Pbf)-Phe-Pro-Asp(OtBu)$\operatorname{Trp}($ Boc $)-\mathrm{NH}_{2}$.

\section{Resin-O-Trp(Boc)-Asp(OtBu)-Arg(Pbf)-Phe-Pro-Asp(OtBu)-Trp(Boc)-Ser(Trt)-NH} Resin-O-Trp(Boc)-Asp(OtBu)-Arg(Pbf)-Phe-Pro-Asp(OtBu)-Trp(Boc)-Ser(Trt)- $\mathrm{NH}_{2}$ was synthesized following the Coupling Reaction procedure, using Resin-O-Trp(Boc)-Asp(OtBu)-Arg(Pbf)-Phe-Pro$\mathrm{Asp}(\mathrm{OtBu})-\operatorname{Trp}(\mathrm{Boc})-\mathrm{NH}_{2}$ from the previous reaction, $0.24 \mathrm{~g}$ Fmoc-Ser(Trt)-OH (0.43 mmol, 2 equiv.), $0.058 \mathrm{~g} \mathrm{HOBt}$ (0.43 mmol, 2 equiv.), $0.13 \mathrm{~mL}$ DIC (0.86 mmol, 4 equiv.), and $1.3 \mathrm{~mL} \mathrm{DMF}$ to generate a concentration of $0.3 \mathrm{M}$. The coupling reaction was run for $2 \mathrm{~h}$ and a negative ninhydrin test was used to confirm reaction completion. The reaction mixture was drained to afford Resin-O-Trp(Boc)-Asp(OtBu)$\operatorname{Arg}(\mathrm{Pbf})-\mathrm{Phe}-\mathrm{Pro}-\mathrm{Asp}(\mathrm{OtBu})-\operatorname{Trp}(\mathrm{Boc})-\mathrm{Ser}(\mathrm{Trt})-\mathrm{NHFmoc}$. The Fmoc group was then removed following the Fmoc Removal procedure to afford Resin-O-Trp(Boc)-Asp(OtBu)-Arg(Pbf)-Phe-Pro$\operatorname{Asp}(\mathrm{OtBu})-\operatorname{Trp}(\mathrm{Boc})-\mathrm{Ser}(\mathrm{Trt})-\mathrm{NH}_{2}$. 


\section{HO-Trp(Boc)-Asp(OtBu)-Arg(Pbf)-Phe-Pro-Asp(OtBu)-Trp(Boc)-Ser(Trt)-NH}

The protected linear peptide HO-Trp(Boc)-Asp(OtBu)-Arg(Pbf)-Phe-Pro-Asp(OtBu)-Trp(Boc)-Ser(Trt)$\mathrm{NH}_{2}$ was generated following the Resin Cleavage of Linear Peptide procedure. The linear peptide was cleaved from the resin using a solution of $4 \mathrm{~mL}$ trifluoroethanol and $4 \mathrm{~mL} \mathrm{CH}_{2} \mathrm{Cl}_{2}$. The resin containing solution was filtered and dried in vacuo to yield the protected linear peptide as a pale yellow solid (104 $\mathrm{mg}, 25 \%$ overall).

\section{HO-Trp-Asp-Arg-Phe-Pro-Asp-Trp-Ser-NH 2 (Compound 13)}

The deprotected linear peptide was synthesized following the Side Chain Deprotection procedure using $0.085 \mathrm{~g}$ crude HO-Trp(Boc)-Asp(OtBu)-Arg(Pbf)-Phe-Pro-Asp(OtBu)-Trp(Boc)-Ser(Trt)-NH $\mathrm{NH}_{2}(0.044$ mmol, 1 equiv.), $0.43 \mathrm{~mL}$ of a mixed solution of TFA and $\mathrm{CH}_{2} \mathrm{Cl}_{2}(9: 1 \mathrm{v} / \mathrm{v})$ and $0.058 \mathrm{~mL}$ anisole $(0.53$ mmol, 12 equiv.) to remove side chain protecting groups. The reaction mixture was stirred for $24 \mathrm{~h}$ and monitored via LC/MS. Upon completion, the reaction mixture was worked up as described in the Side Chain Deprotection procedure to afford the crude linear peptide. The crude product was purified using HPLC, then lyophilized to generate pure compound as a white solid ( $3 \mathrm{mg}, 2 \%$ overall). The purity of the final compound was found to be $>95 \%$ by ${ }^{1} \mathrm{H}$ NMR, 2-D NMR (COSY, HSQC, HMBC) and LC/MS. LC/MS (ESI) $m / z:[\mathrm{M}+\mathrm{H}]^{+}$calculated for $\mathrm{C}_{53} \mathrm{H}_{65} \mathrm{~N}_{13} \mathrm{O}_{14}{ }^{+}$1108.48, found 1108.50 .

${ }^{1} \mathrm{H}$ NMR (600 MHz, D $\left.2 \mathrm{O}\right): \delta 7.69$ (d, $J=8.04 \mathrm{~Hz}, 1 \mathrm{H}$, ArH Trp), 7.60 (d, $\left.J=8.04 \mathrm{~Hz}, 1 \mathrm{H}, \operatorname{ArH} \operatorname{Trp}\right)$, 7.46 (t, $J=8.04 \mathrm{~Hz}, 2 \mathrm{H}, \operatorname{ArH} \operatorname{Trp}), 7.33$ (t, $J=7.75 \mathrm{~Hz}, 2 \mathrm{H}$, ArH Phe), 7.30-7.11 (m, 9H, ArH Trp, ArH Phe), 4.74 (dd, $J=8.55,5.93 \mathrm{~Hz}, 1 \mathrm{H}, \alpha \mathrm{H} \mathrm{Asp}), 4.68$ (t, $J=7.8 \mathrm{~Hz}, 1 \mathrm{H}, \alpha \mathrm{H}$ Trp), 4.63 (dd, $J=7.75,5.13$ Hz, 1H, $\alpha \mathrm{H}$ Asp), 4.54-4.47 (m, 2H, $\alpha \mathrm{H}$ Trp, $\alpha \mathrm{H}$ Phe), 4.13 (dd, J= 5.59, 4.56 Hz, 1H, $\alpha \mathrm{H}$ Ser), 4.08 (t, $J=7.05 \mathrm{~Hz}, 1 \mathrm{H}, \alpha \mathrm{H}$ Arg), 4.00-3.89 (m, 3H, $\alpha \mathrm{H}$ Pro, $\beta \mathrm{H}$ Ser), 3.58-3.53 (m, 1H, $\delta \mathrm{H}$ Pro), 3.35-3.13 (m, 6H, $\beta \mathrm{H}$ Trp, $\beta \mathrm{H}$ Trp, $\delta \mathrm{H}$ Pro, $\beta \mathrm{H}$ Phe), 2.97 (dd, $J=13.93,10.2 \mathrm{~Hz}, 1 \mathrm{H}, \beta \mathrm{H}$ Phe), 2.88-2.78 (m, $2 \mathrm{H}, \delta \mathrm{H}$ Arg), 2.77-2.37 (m, 4H, $\beta \mathrm{H}$ Asp, $\beta \mathrm{H}$ Asp), 2.09-1.97 (m, 1H, $\beta \mathrm{H}$ Pro), 1.75-1.65 (m, 1H, $\gamma \mathrm{H}$ Pro), 1.571.37 (m, 4H, $\beta \mathrm{H}$ Pro, $\gamma \mathrm{H}$ Pro, $\beta \mathrm{H}$ Arg), 1.27-1.13 (m, 2H, $\gamma \mathrm{H}$ Arg). 


\section{LC/MS of Peptide A}<smiles>N=C(N)NCCC[C@H](NC(=O)[C@H](Cc1ccccc1)NC(=O)[C@@H]1CCCN1C(=O)[C@H](CC(=O)O)NC(=O)[C@H](Cc1c[nH]c2ccccc12)NC(=O)[C@H](CO)NC(=O)[C@@H]1CCCN1C(=O)CN)C(=O)N[C@@H](CC(=O)O)C(=O)N[C@@H](Cc1c[nH]c2ccccc12)C(=O)O</smiles>

Chemical Formula: $\mathrm{C}_{60} \mathrm{H}_{75} \mathrm{~N}_{15} \mathrm{O}_{16}$

Exact Mass: 1261.55

Molecular Weight: 1262.35

mAU
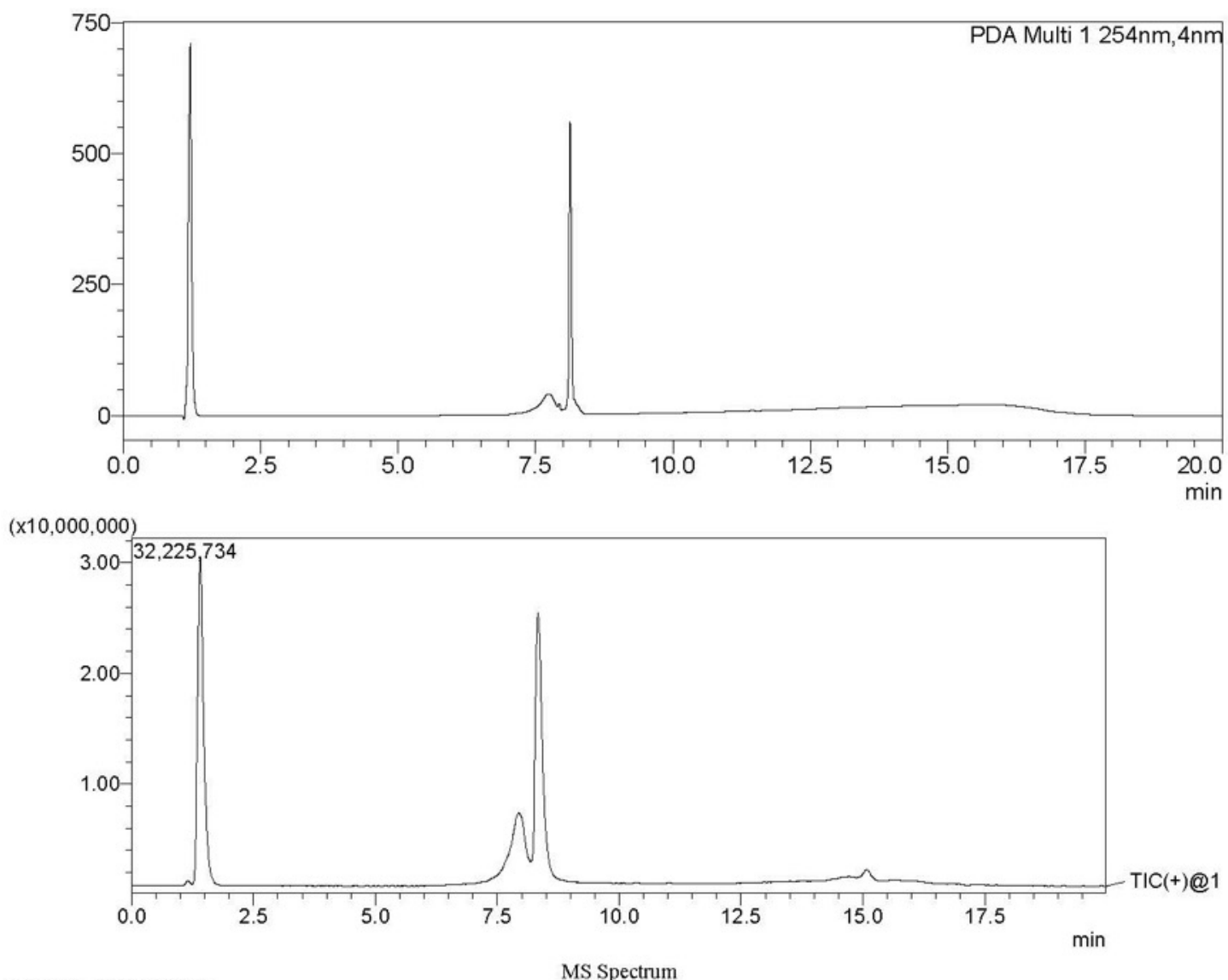

R.Time:1.400(Scan\#:85)

MS Spectrum

MassPeaks:1904

Segment 1 - Event

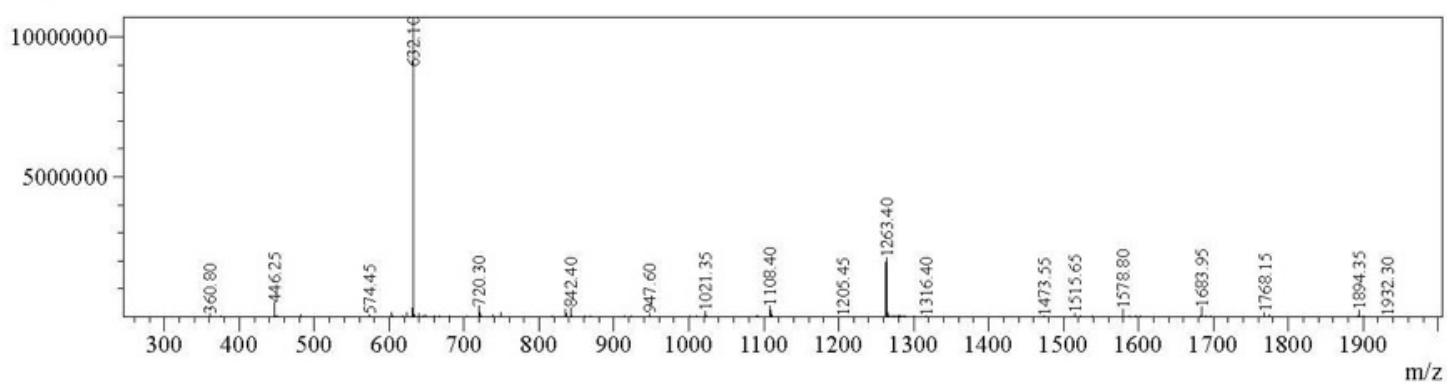


R. Time:7.933(Scan\#:477)

MS Spectrum

MassPeaks:1908

Segment 1 - Event 1

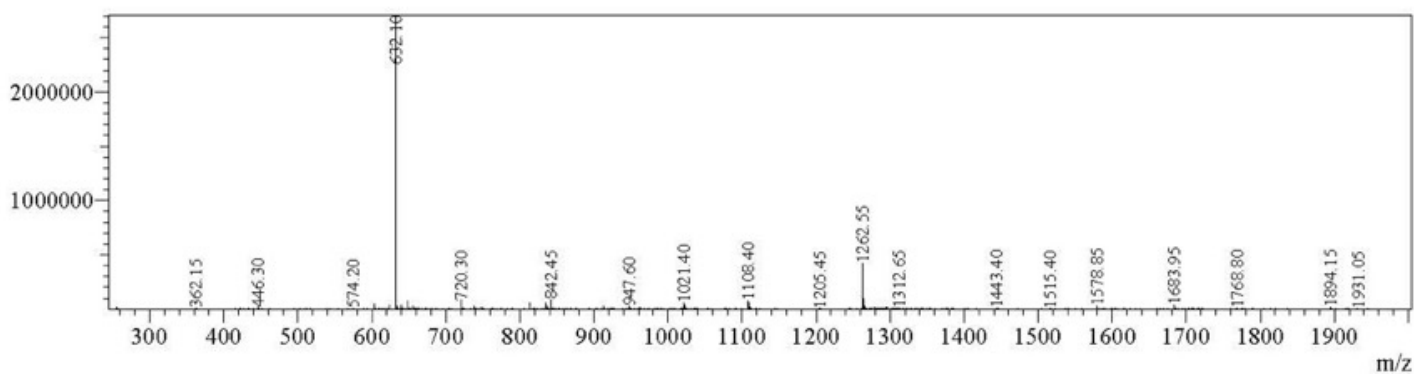

R.Time:8.317(Scan\#:500)

MS Spectrum

MassPeaks: 1904

Segment 1 - Event 1

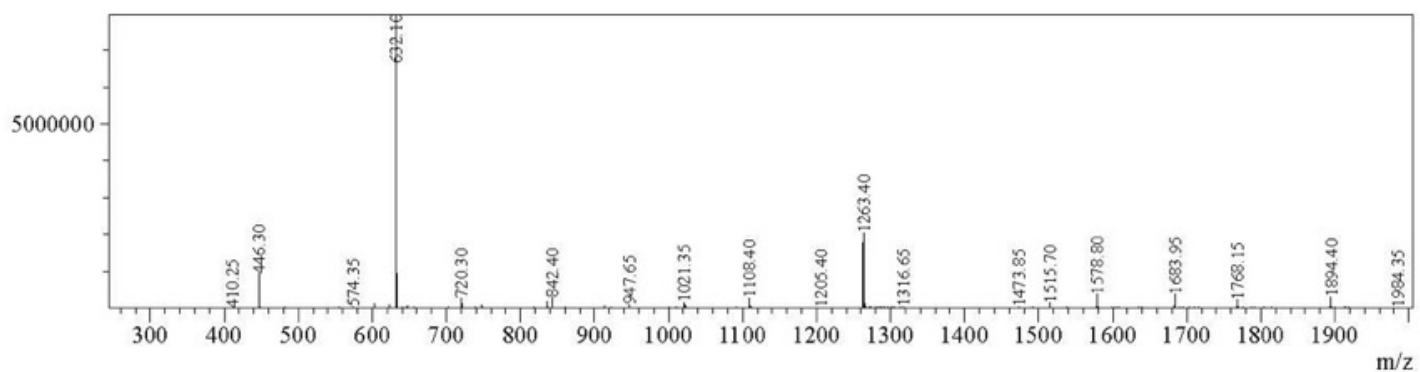




\section{${ }^{1} \mathrm{H}$ NMR of Peptide A}

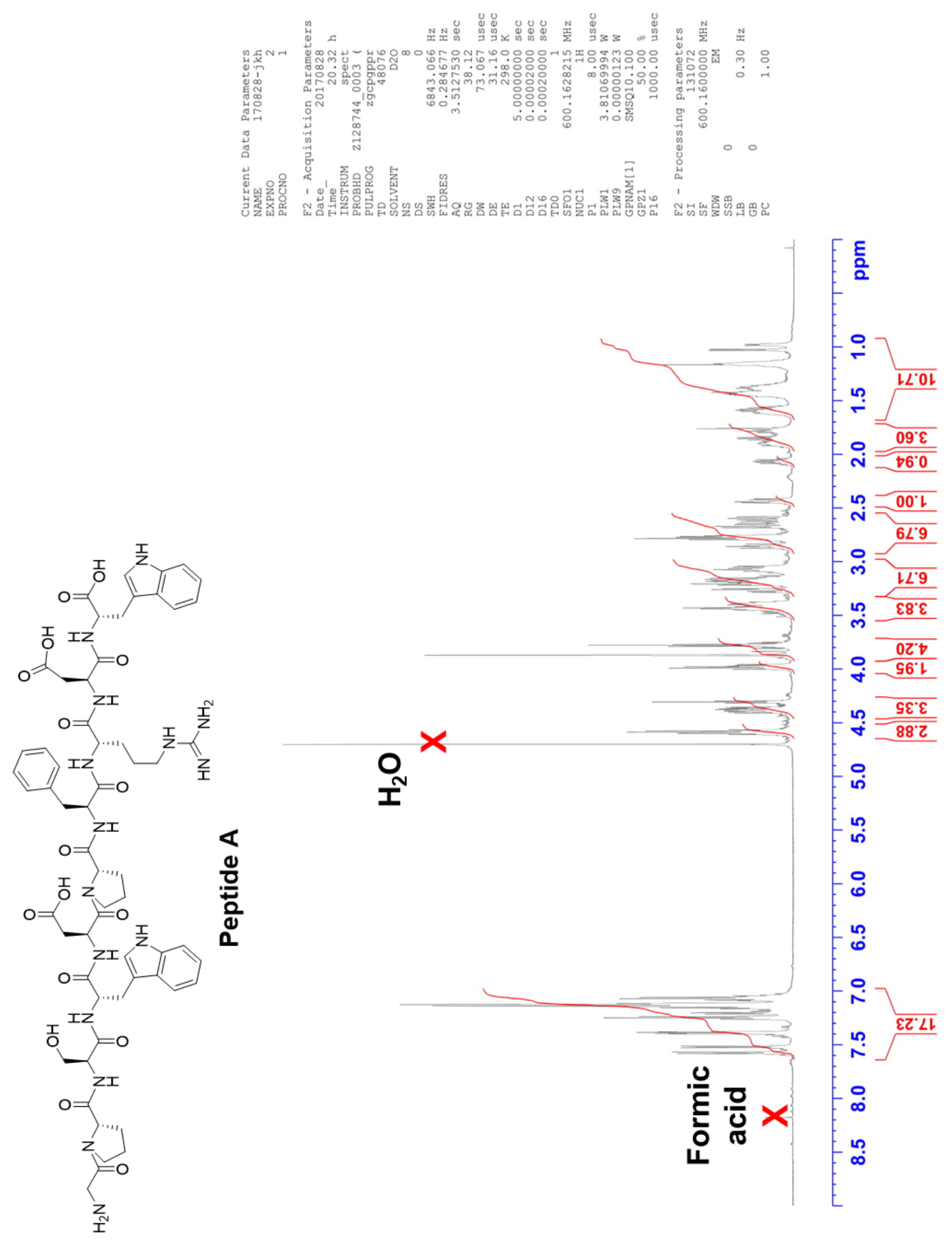




\section{${ }^{1} \mathrm{H}-{ }^{1} \mathrm{H}$ COSY of Peptide A}
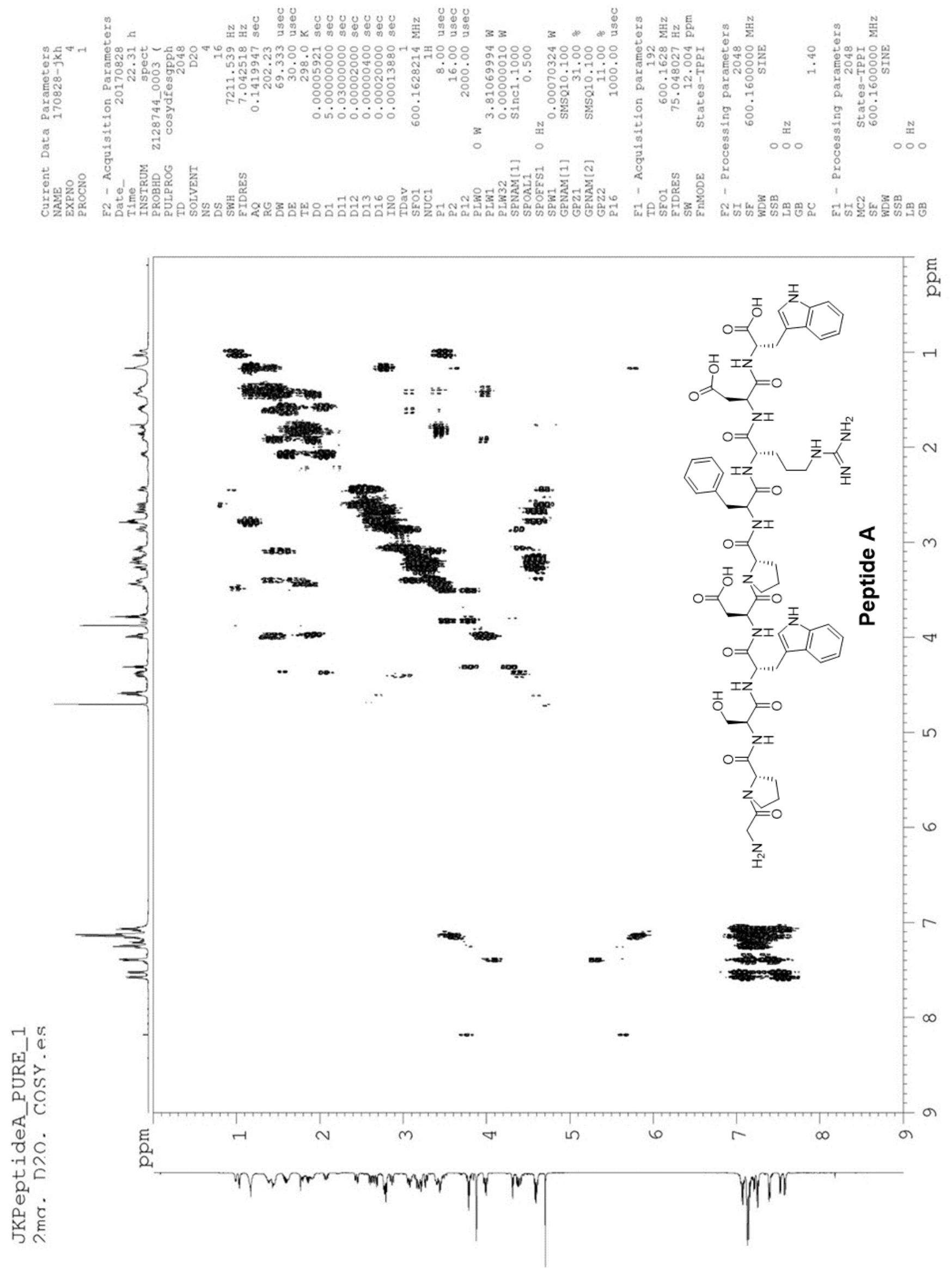


\section{${ }^{1} \mathrm{H}-{ }^{13} \mathrm{C}$ HSQC of Peptide A}

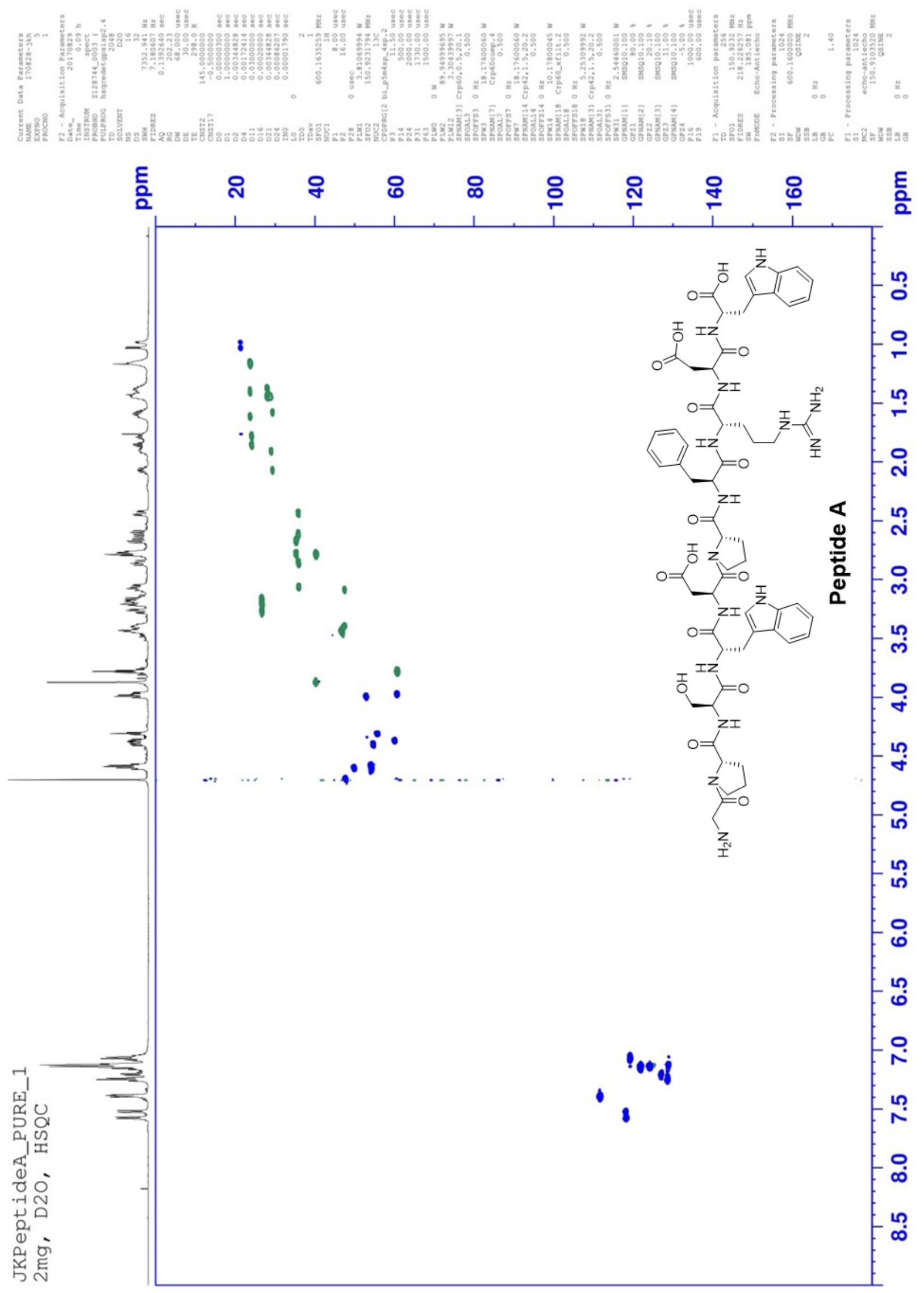




\section{${ }^{1} \mathrm{H}-{ }^{13} \mathrm{C}$ HMBC of Peptide A}

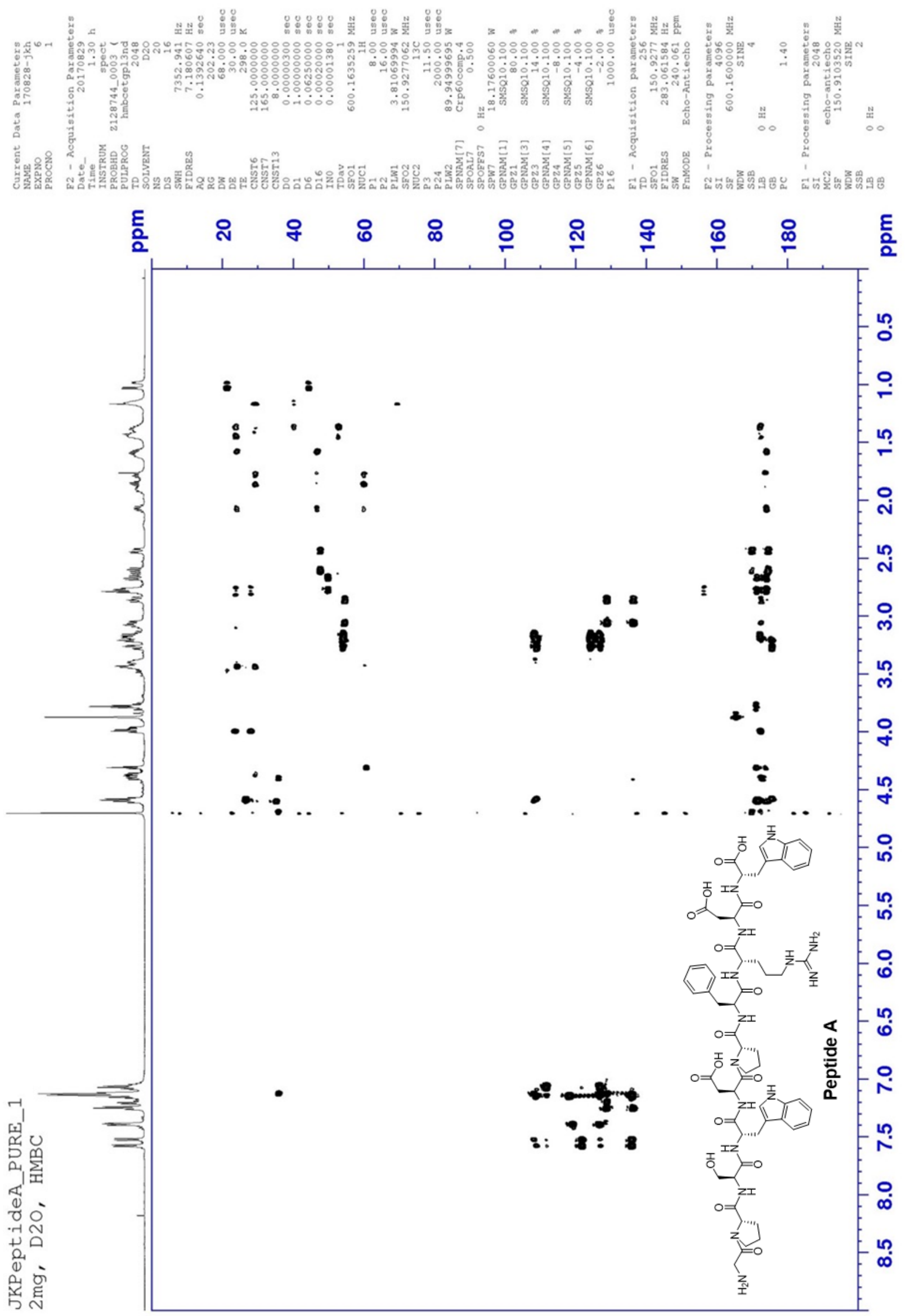




\section{LC/MS of Peptide B}

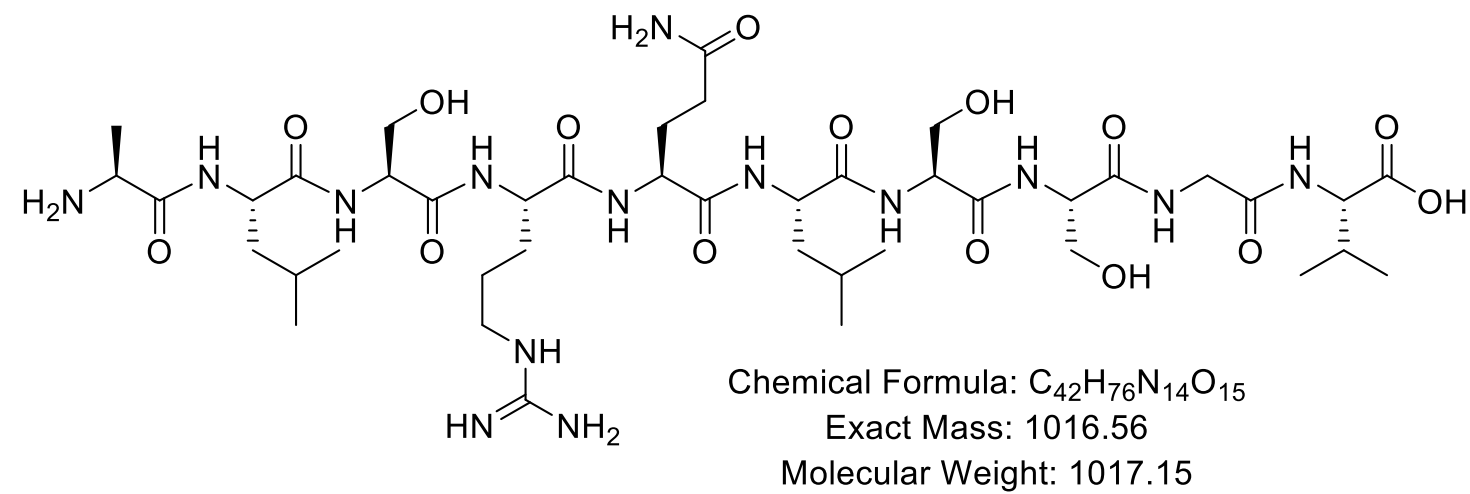

$\mathrm{mAU}$

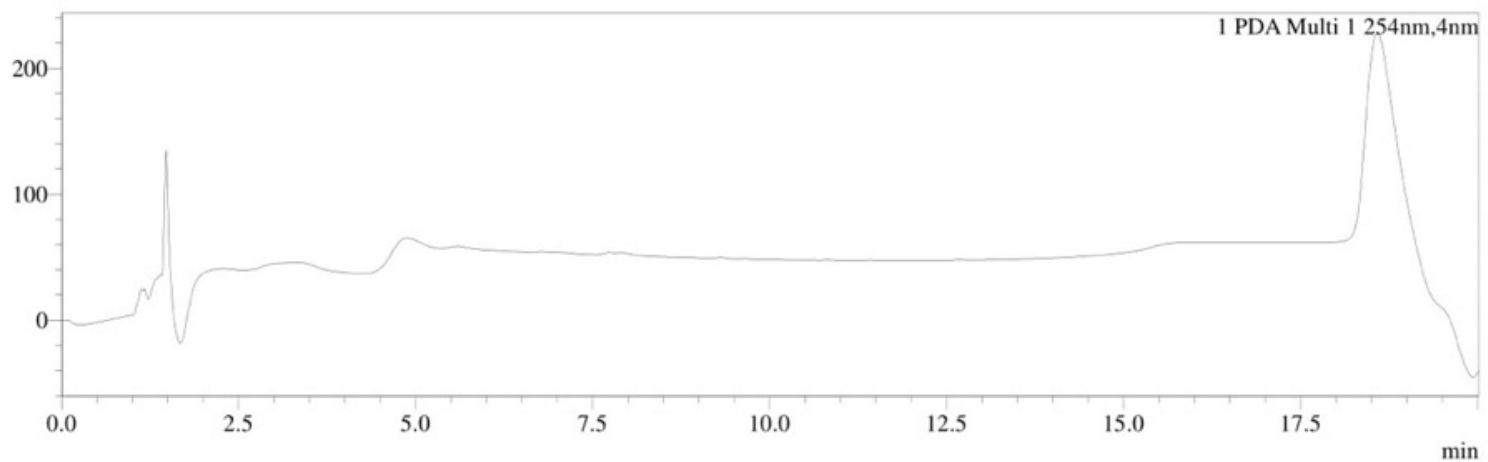

(x100,000,000)

Intensity

TIC(+)@1

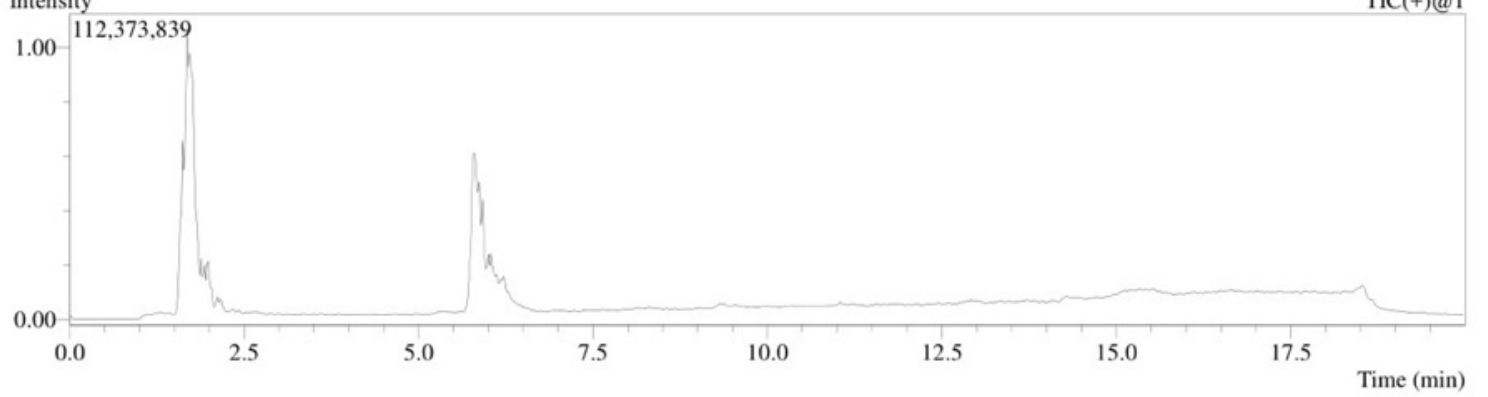

R.Time:1.700(Scan\#:103

MS Spectrum

Segment 1 - Event

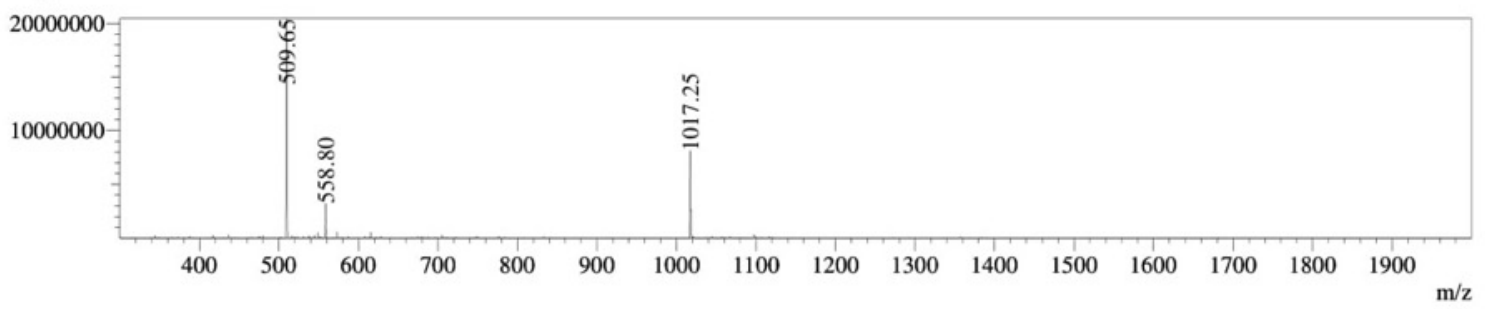

MS Spectrum

R.Time:5.767(Scan\#:347)

MassPeaks:2

Segment 1 - Event 1

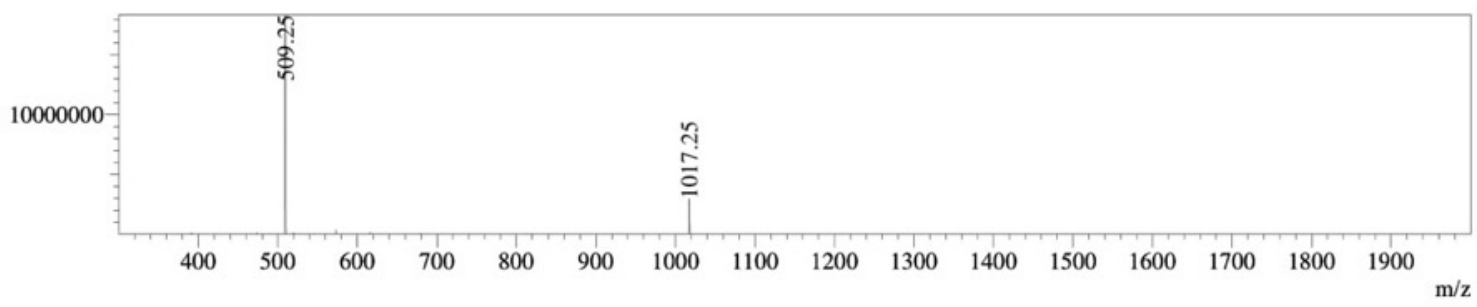




\section{${ }^{1} \mathrm{H}$ NMR of Peptide B}

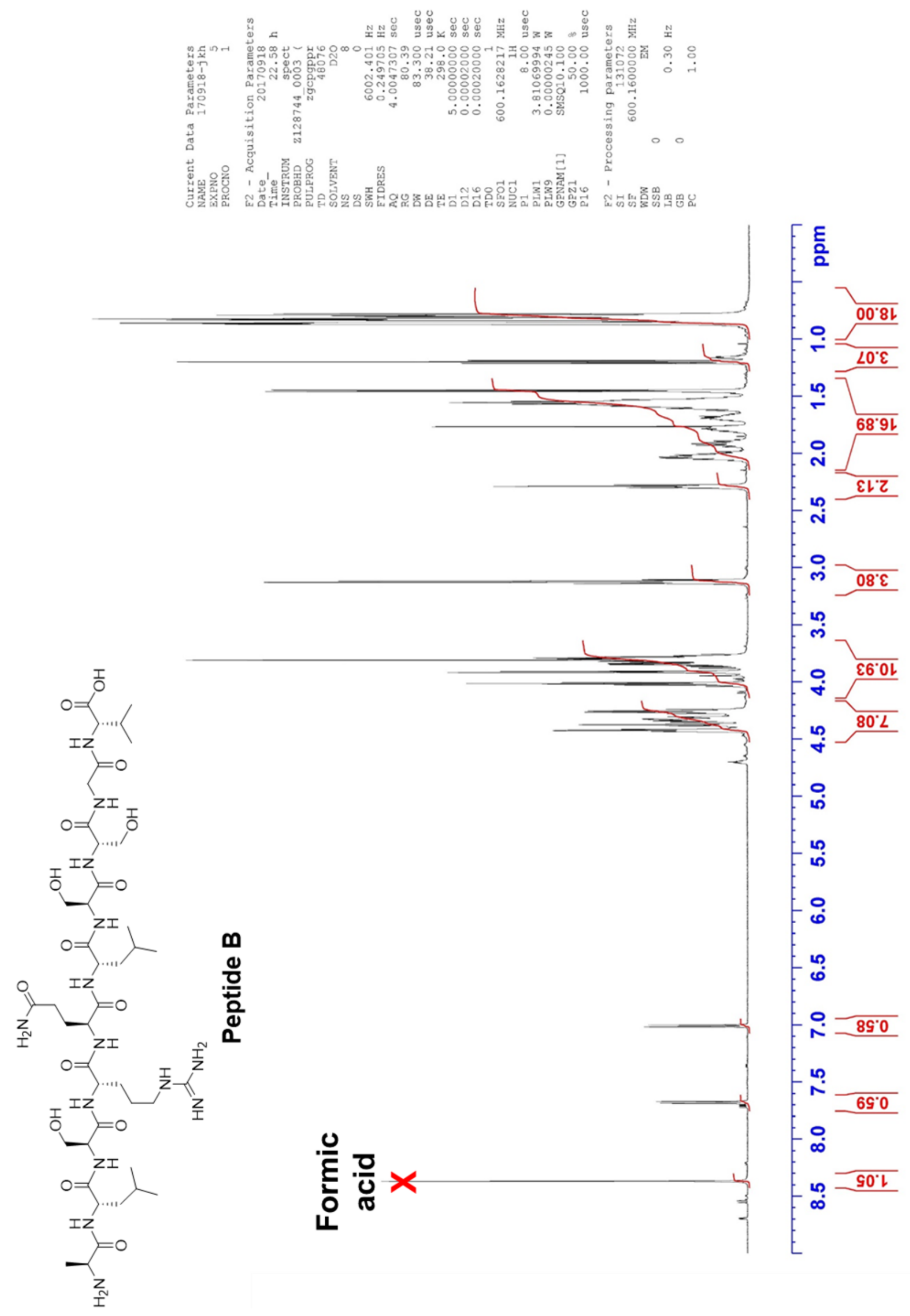




\section{${ }^{1} \mathrm{H}-{ }^{1} \mathrm{H}$ COSY of Peptide B}
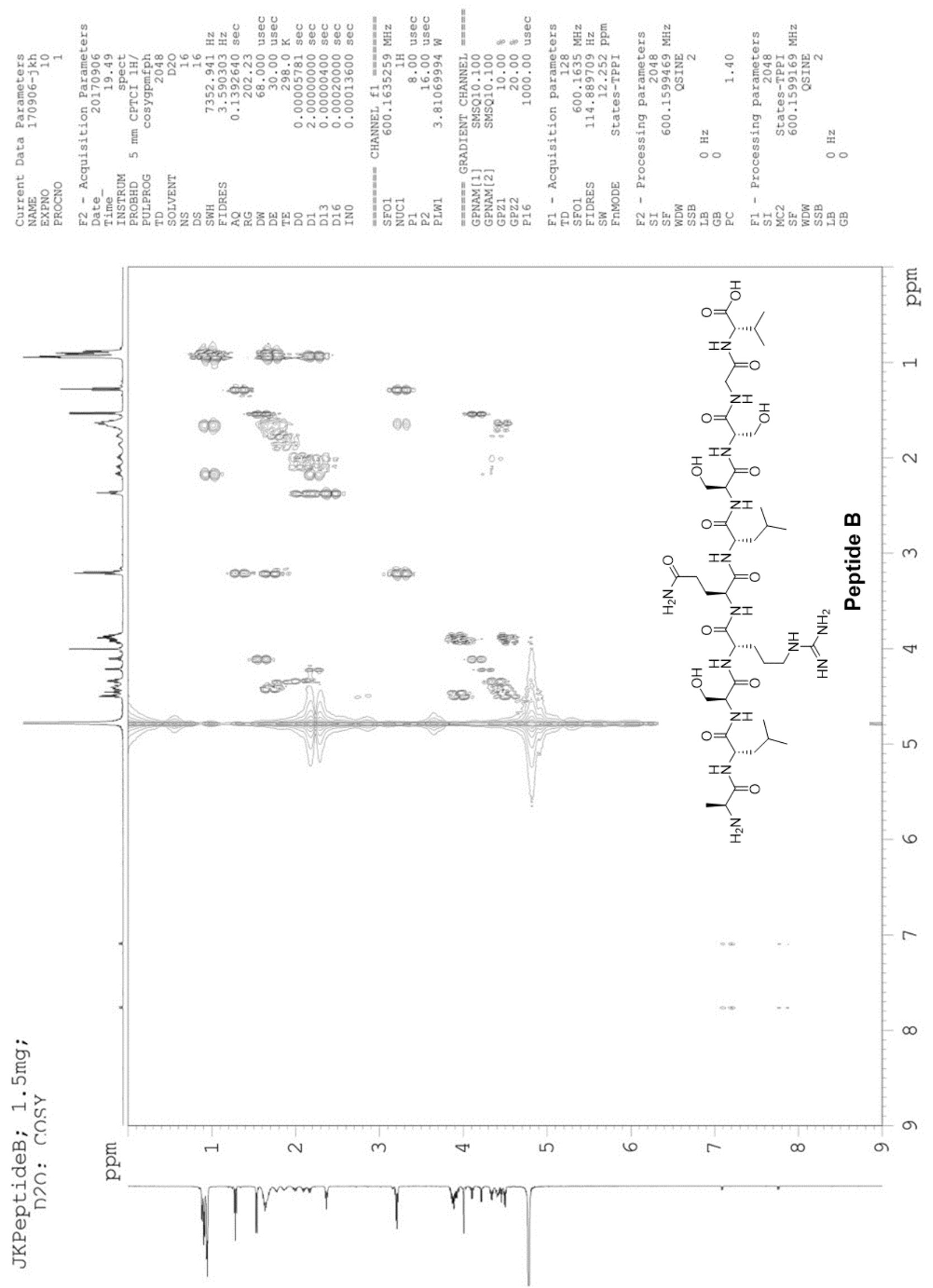


\section{${ }^{1} \mathrm{H}-{ }^{13} \mathrm{C}$ HSQC of Peptide B}

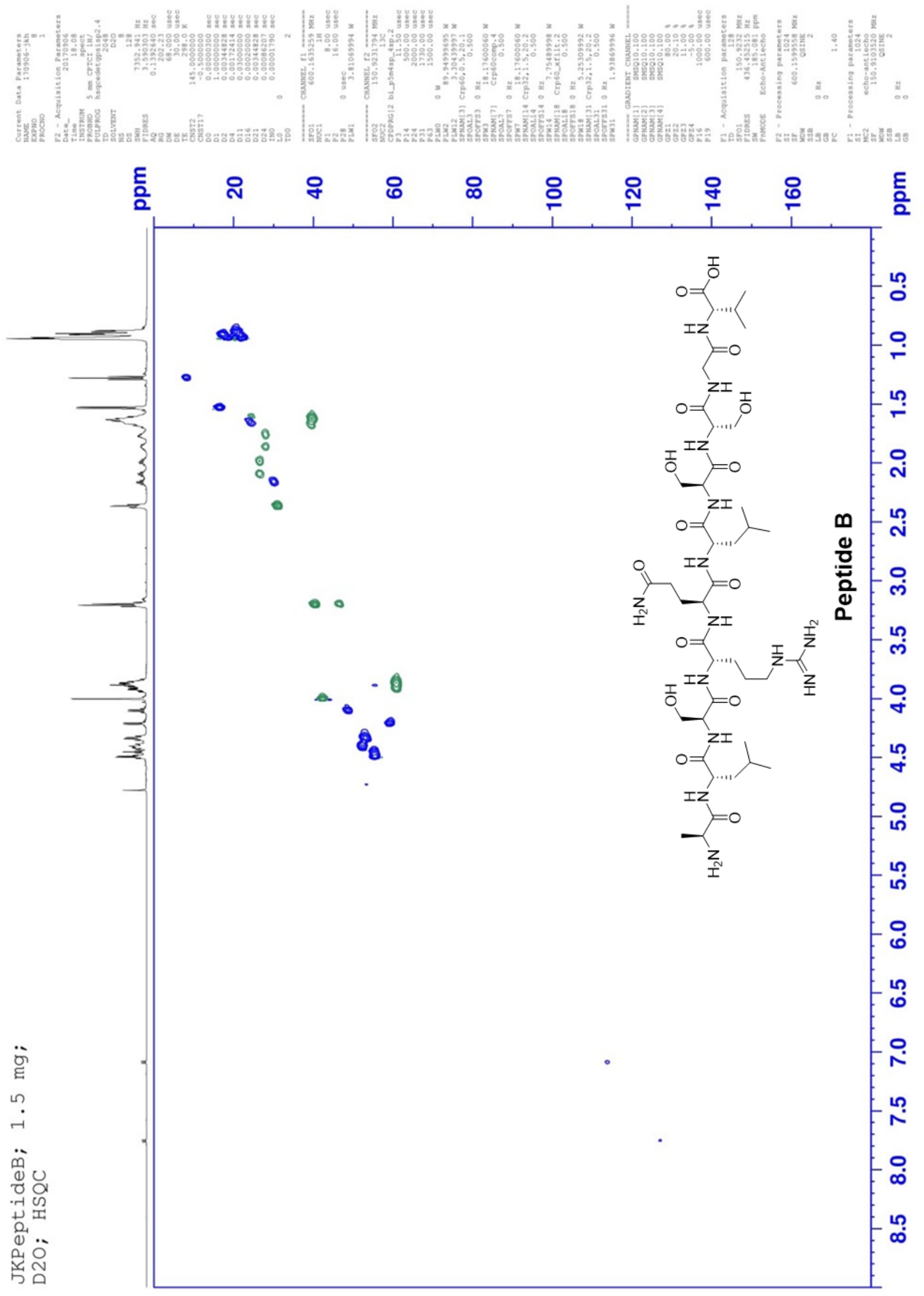




\section{${ }^{1} \mathrm{H}-{ }^{13} \mathrm{C}$ HMBC of Peptide B}

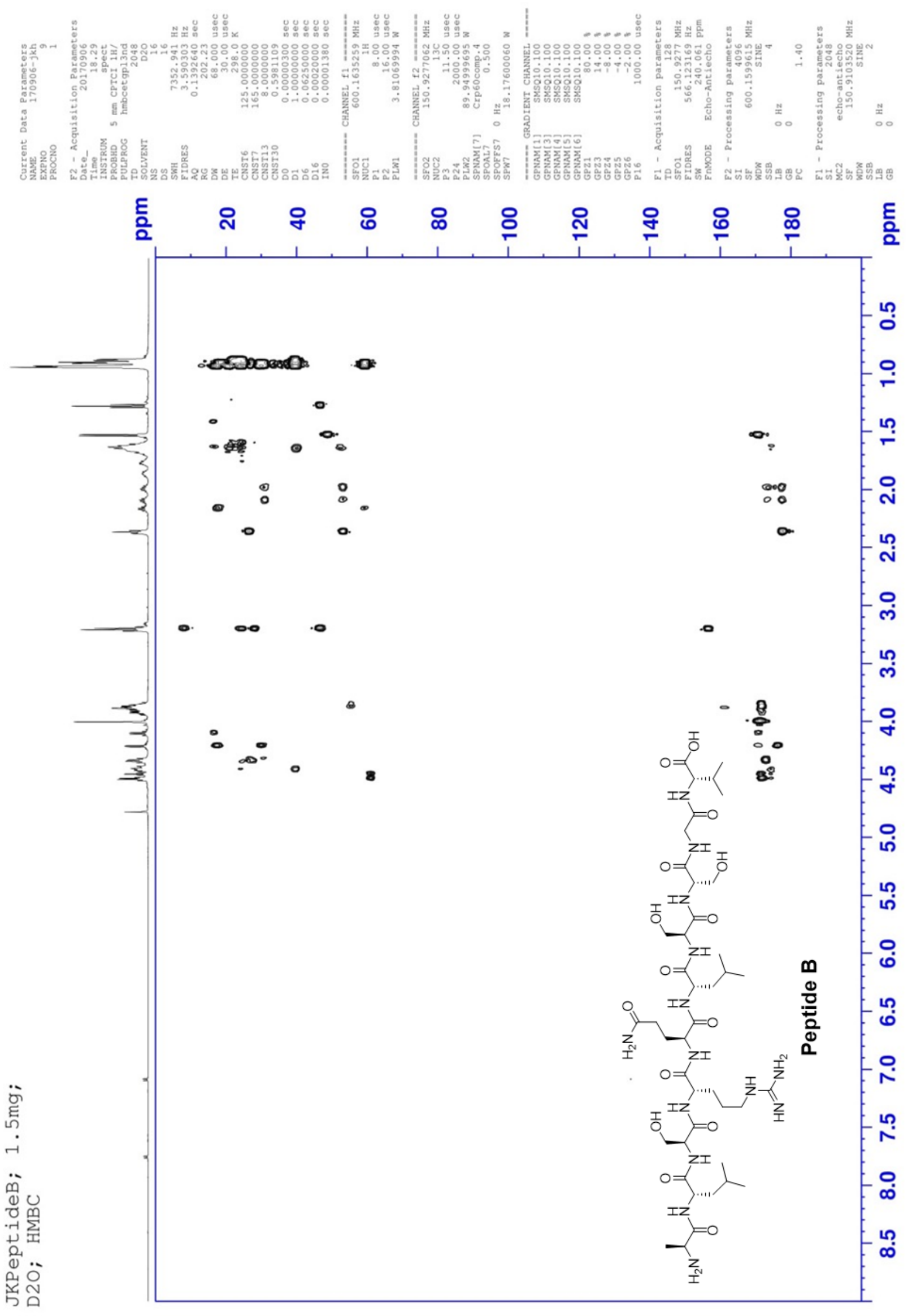




\section{LC/MS of Peptide C}

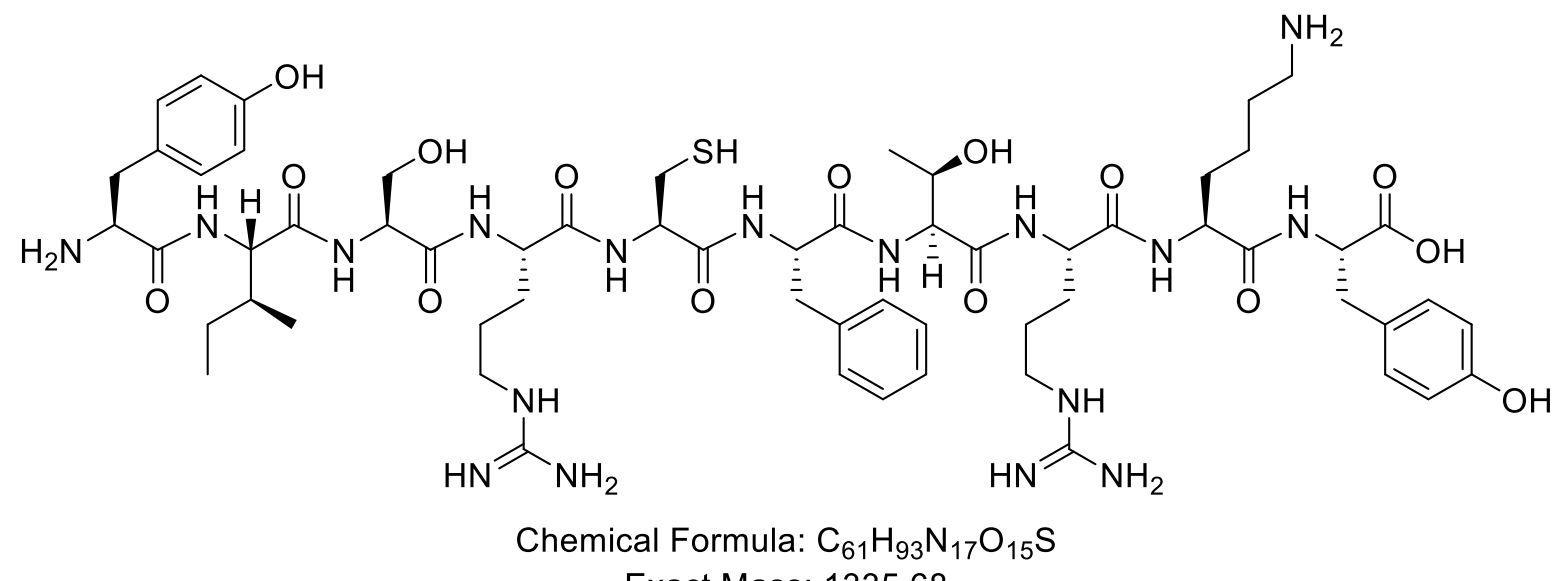

Exact Mass: 1335.68

Molecular Weight: 1336.58

mAU
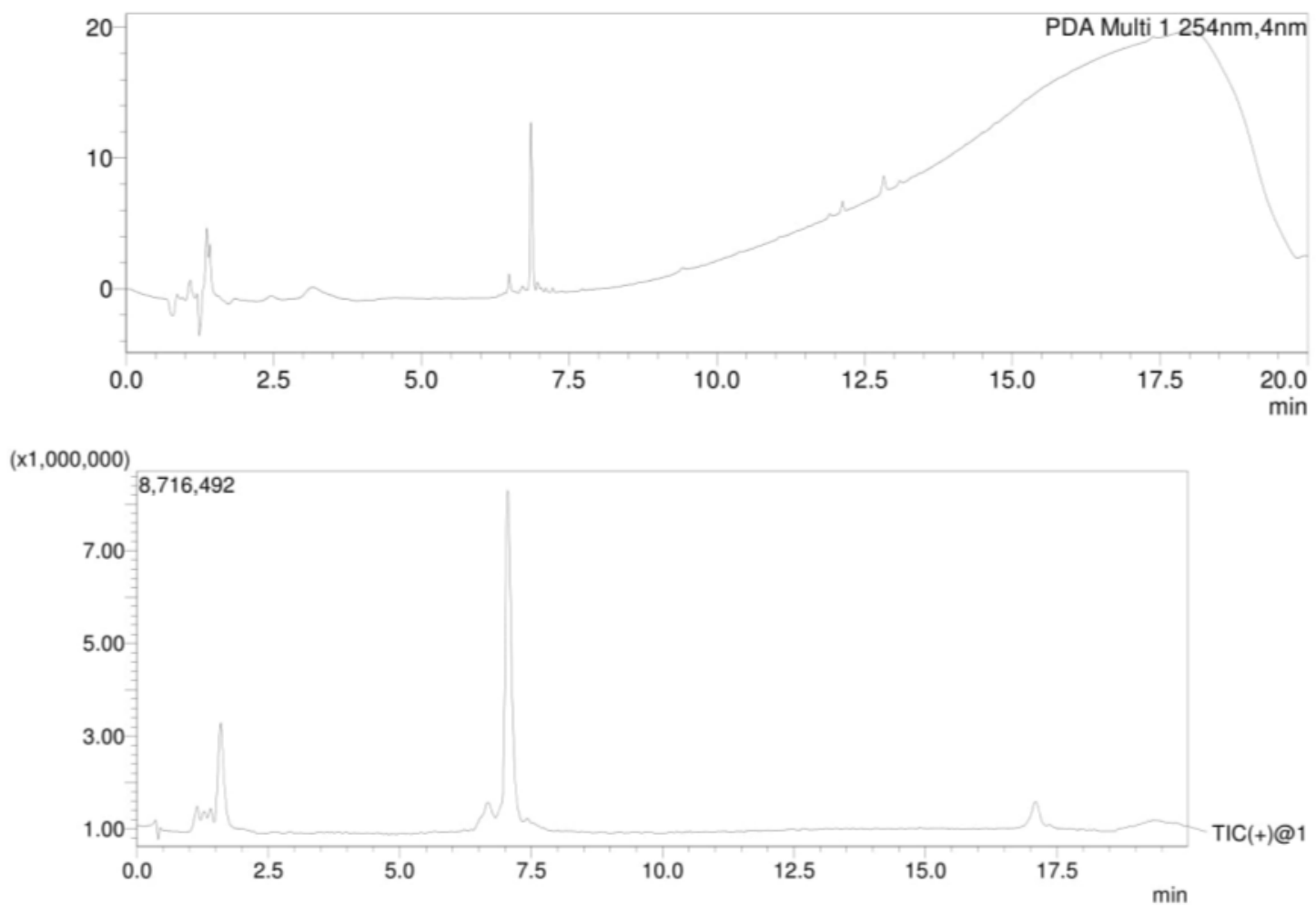

R.Time:7.033(Scan\#:423)

MS Spectrum

Segment I - Event

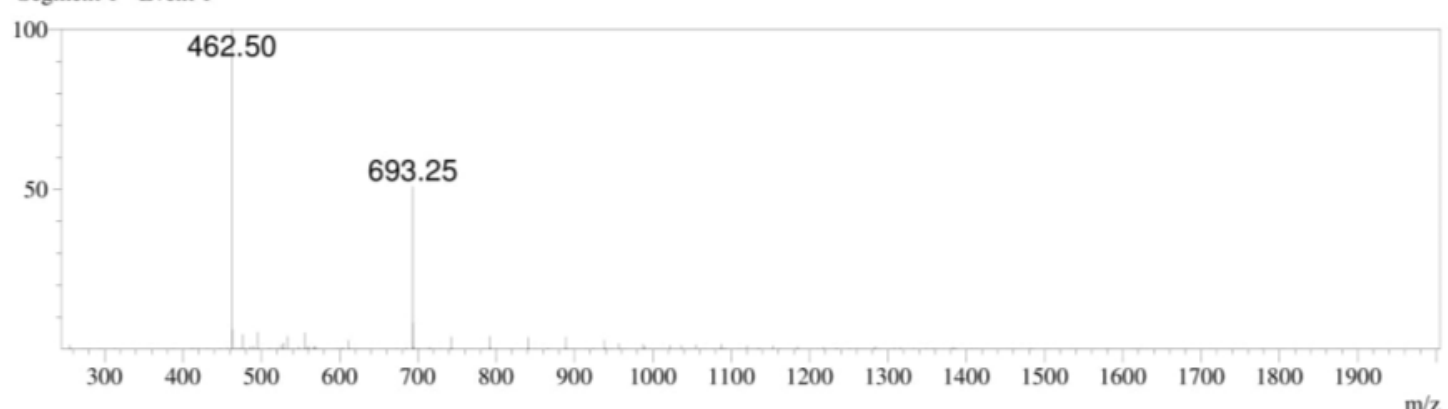




\section{${ }^{1} \mathrm{H}$ NMR of Peptide C}
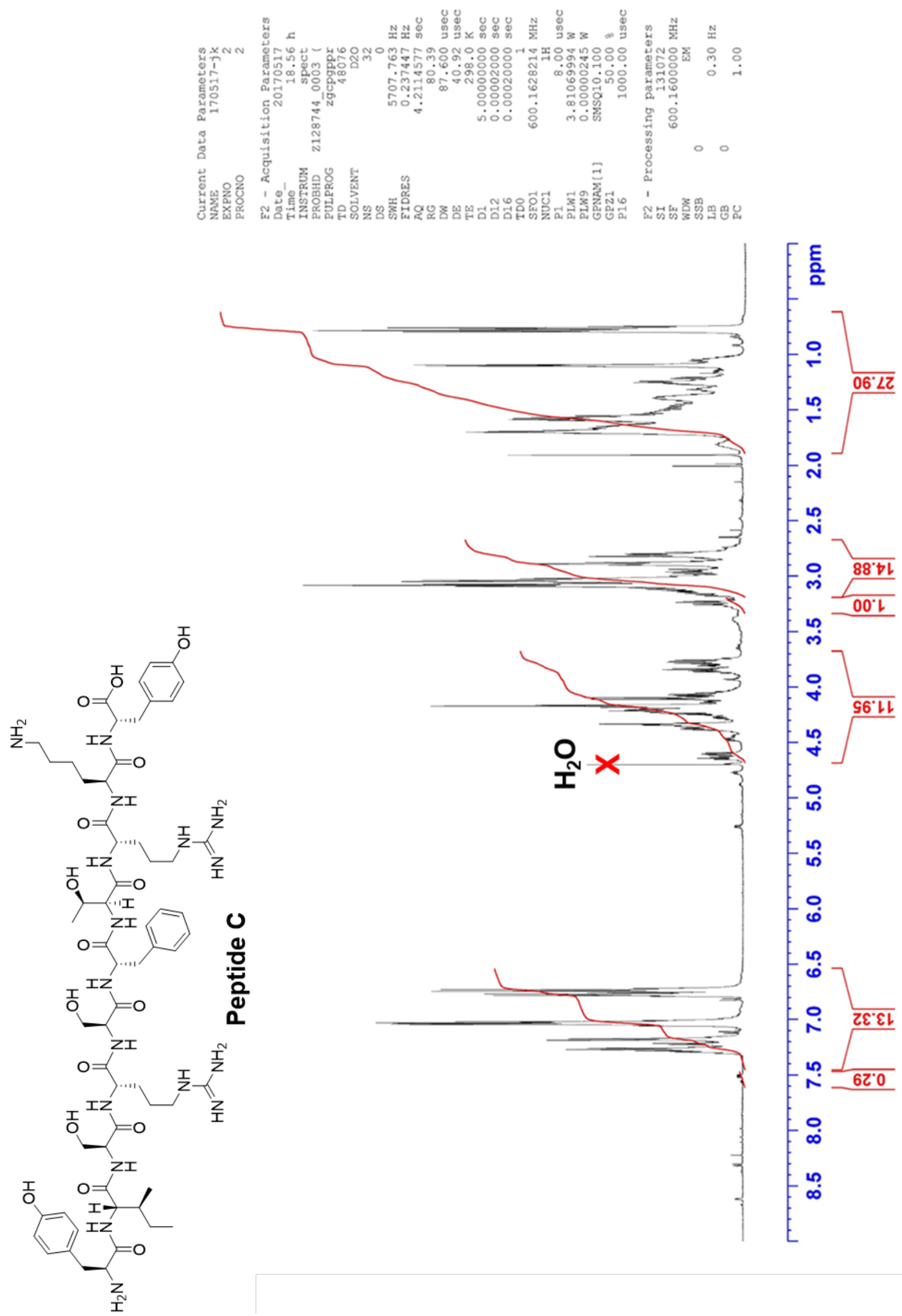


\section{${ }^{1} \mathrm{H}-{ }^{1} \mathrm{H}$ COSY of Peptide C}
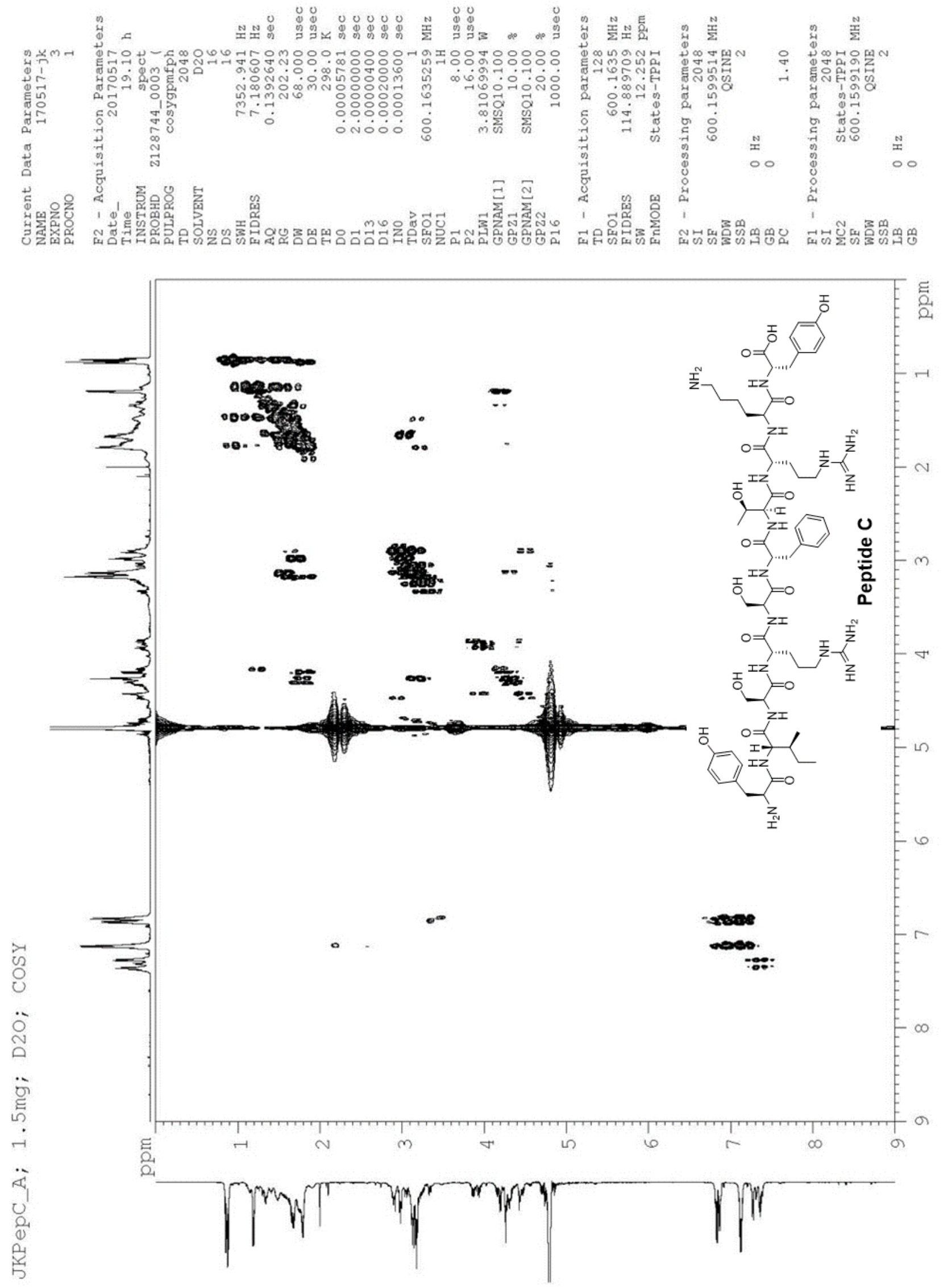


\section{${ }^{1} \mathrm{H}-{ }^{13} \mathrm{C}$ HSQC of Peptide C}

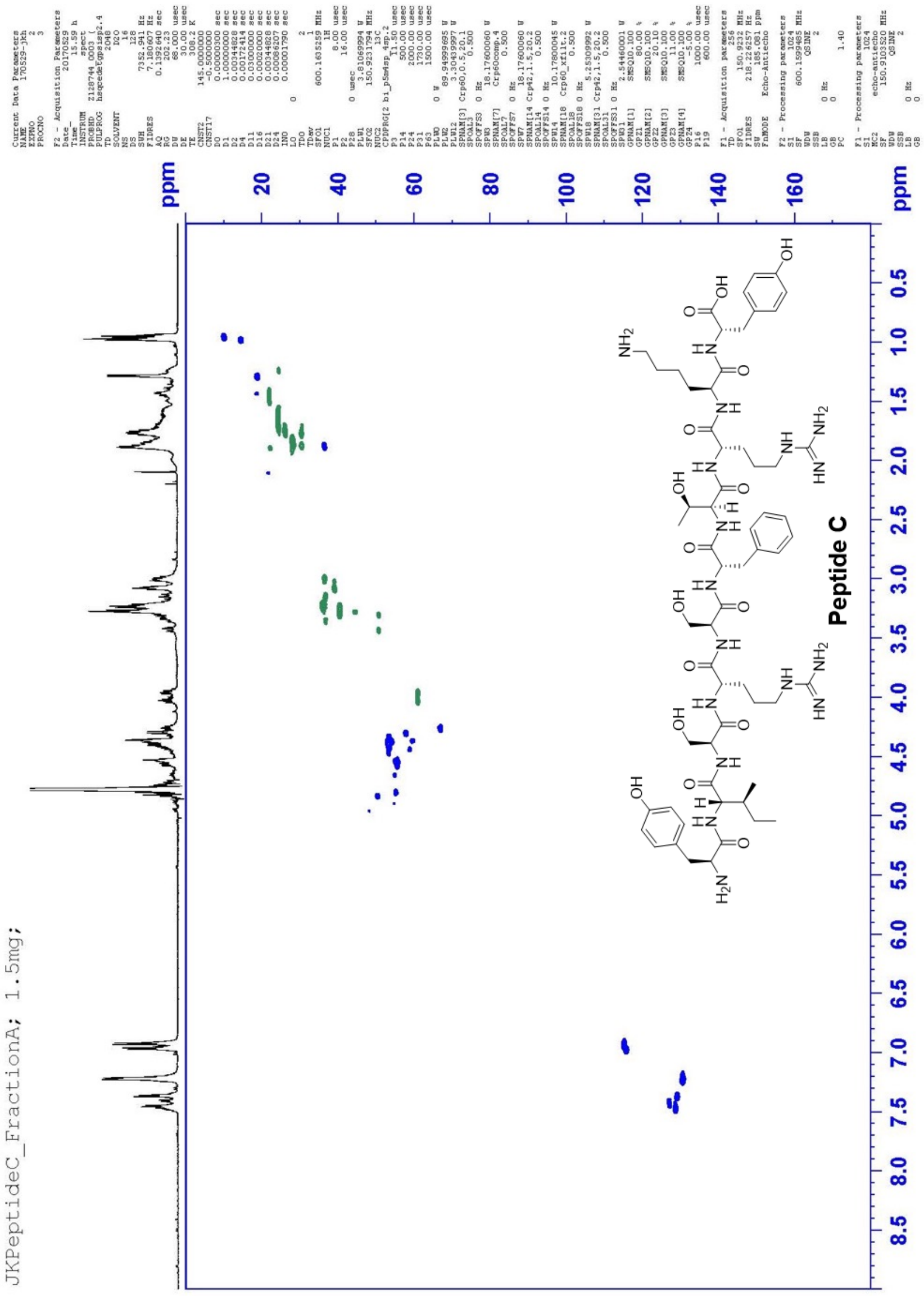




\section{${ }^{1} \mathrm{H}-{ }^{13} \mathrm{C}$ HMBC of Peptide C}

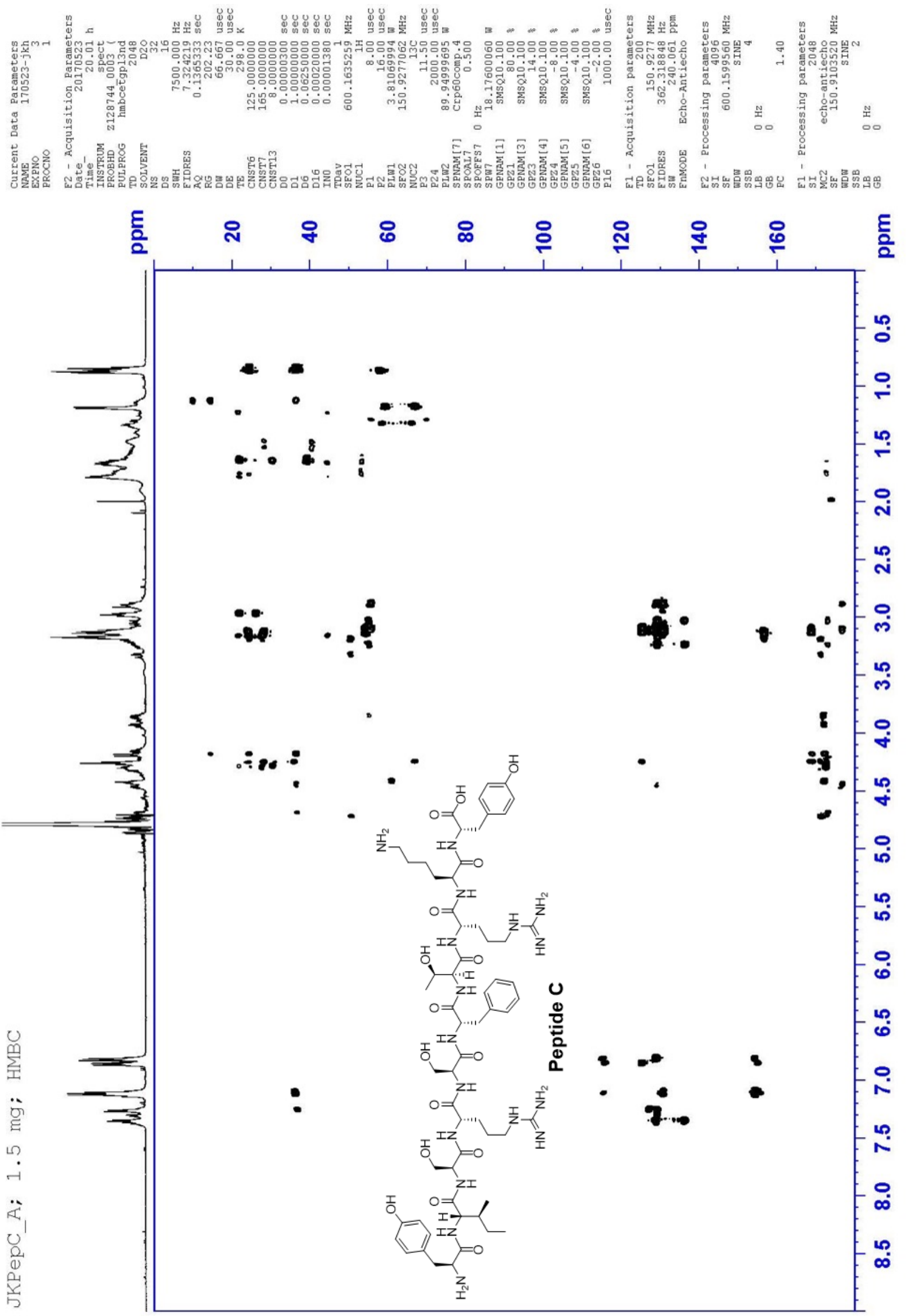




\section{LC/MS of Peptide D}<smiles>CC(C)C[C@H](NC(=O)[C@H](C)N)C(=O)N[C@@H](CO)C(=O)N[C@@H](CCCNC(=N)N)C(=O)N[C@@H](CCC(N)=O)C(=O)N[C@@H](CC(C)C)C(=O)N[C@@H](CO)C(=O)N[C@@H](CO)C(=O)NCC(=O)N[C@@H](C(=O)O)C(C)C</smiles>

Chemical Formula: $\mathrm{C}_{42} \mathrm{H}_{76} \mathrm{~N}_{14} \mathrm{O}_{15}$

Exact Mass: 1016.56

Molecular Weight: 1017.15

mAU

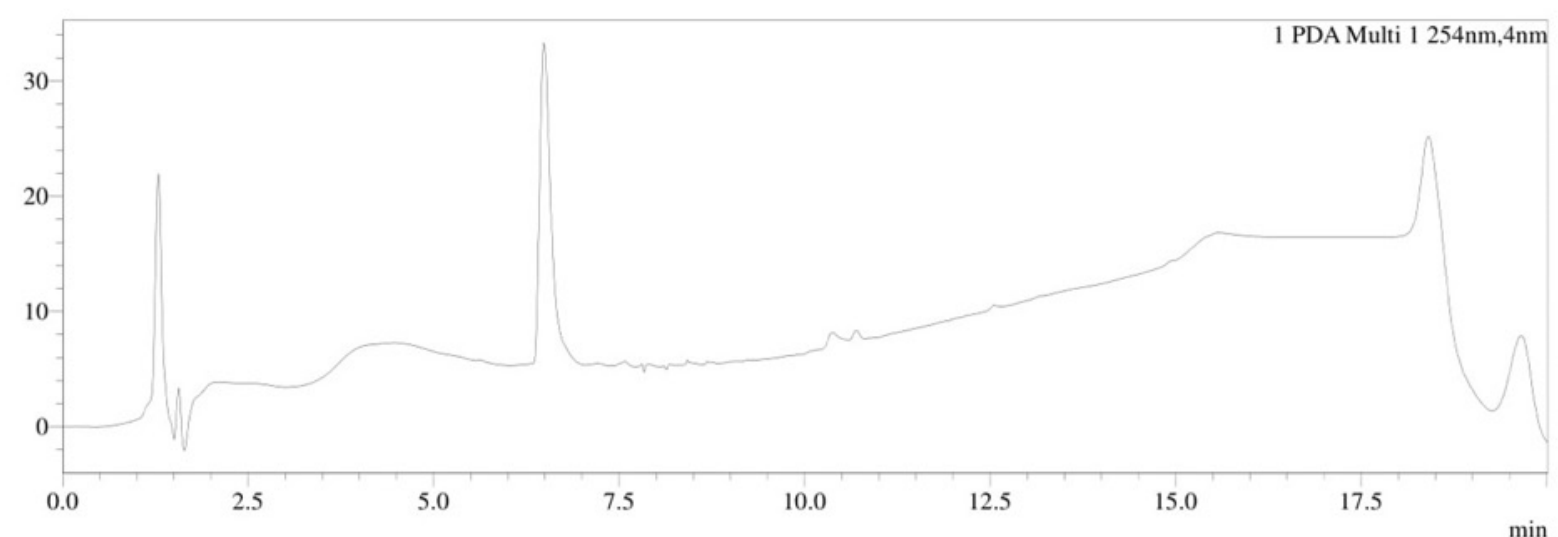

$(\mathrm{x} 10,000,000)$

Intensity

TIC(+)@1

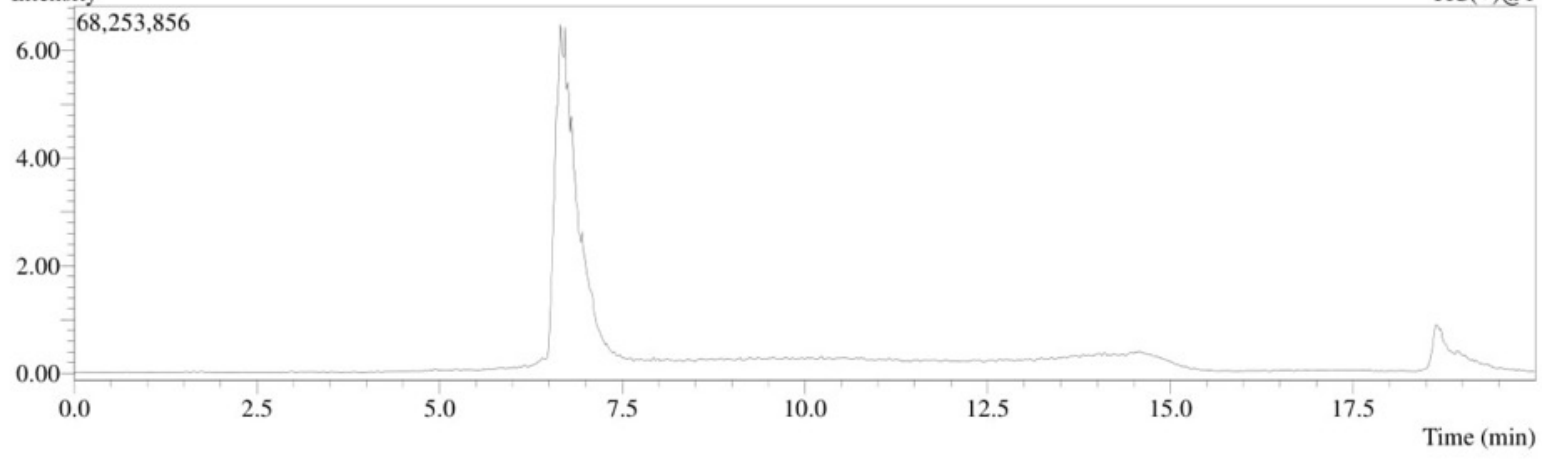

R. Time:6.617(Scan\#:398)

MS Spectrum

MassPeaks:5

Segment 1 - Event 1

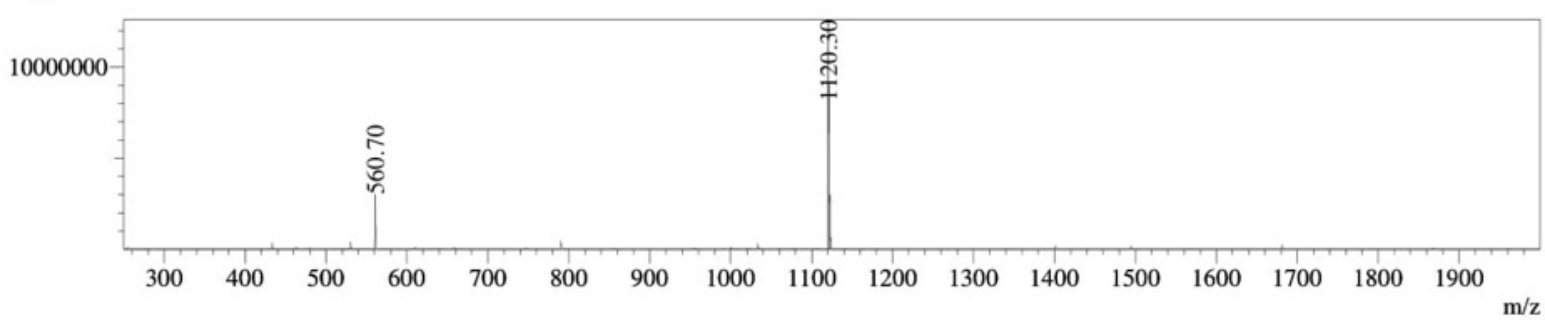




\section{${ }^{1} \mathrm{H}$ NMR of Peptide D}
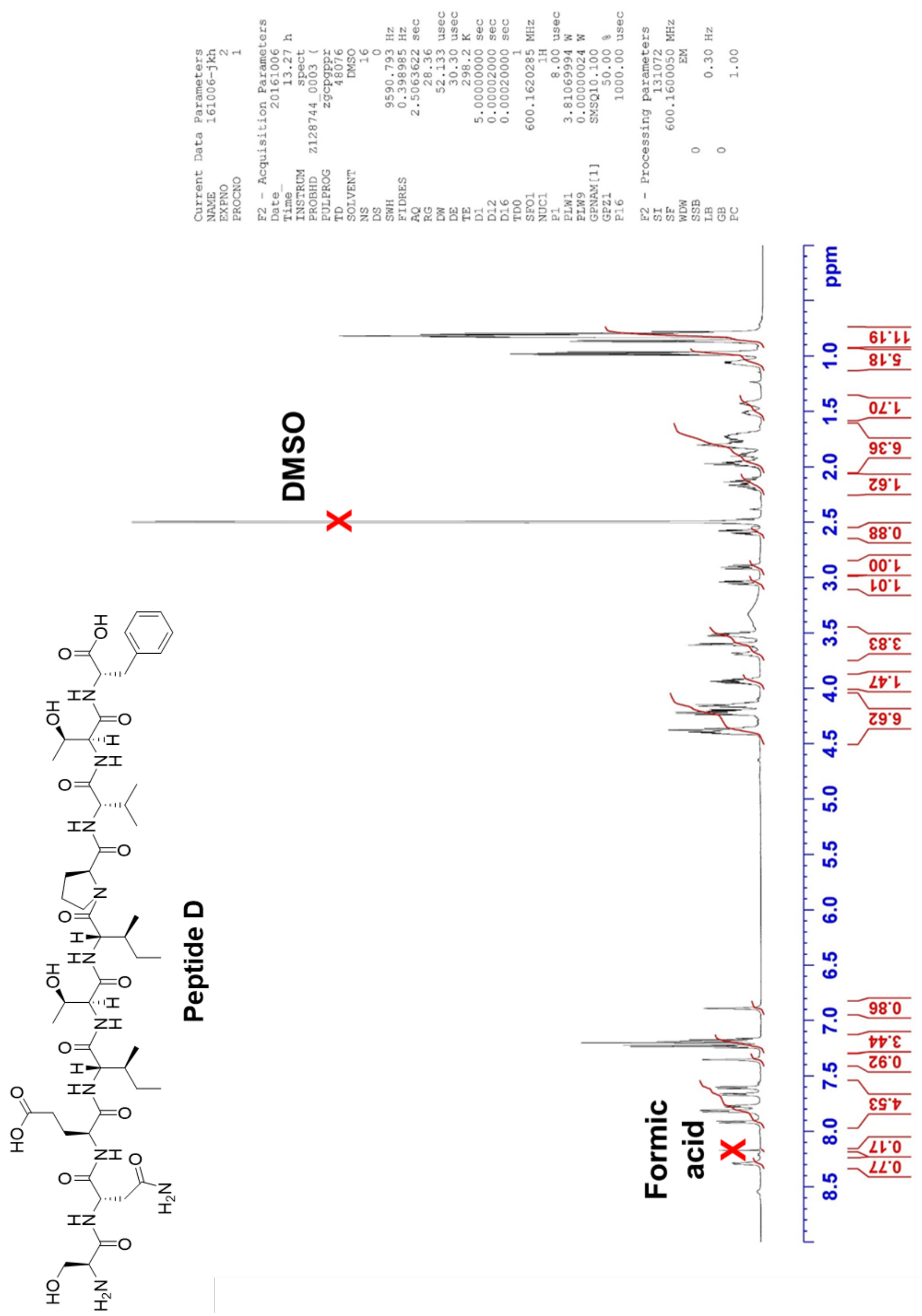


\section{${ }^{1} \mathrm{H}-{ }^{13} \mathrm{C}$ HSQC of Peptide D}

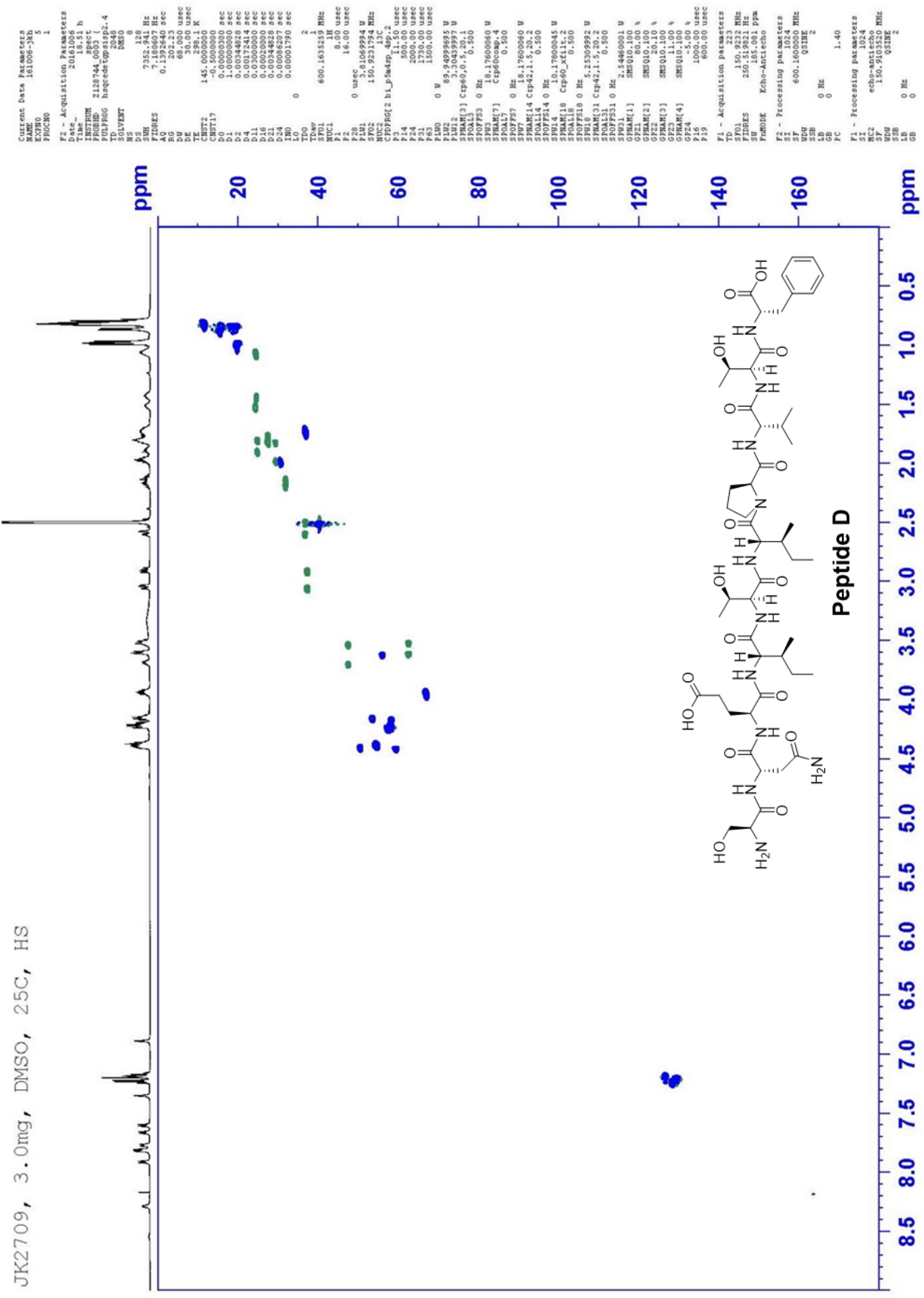




\section{${ }^{1} \mathrm{H}-{ }^{1} \mathrm{H}$ COSY of Peptide D}

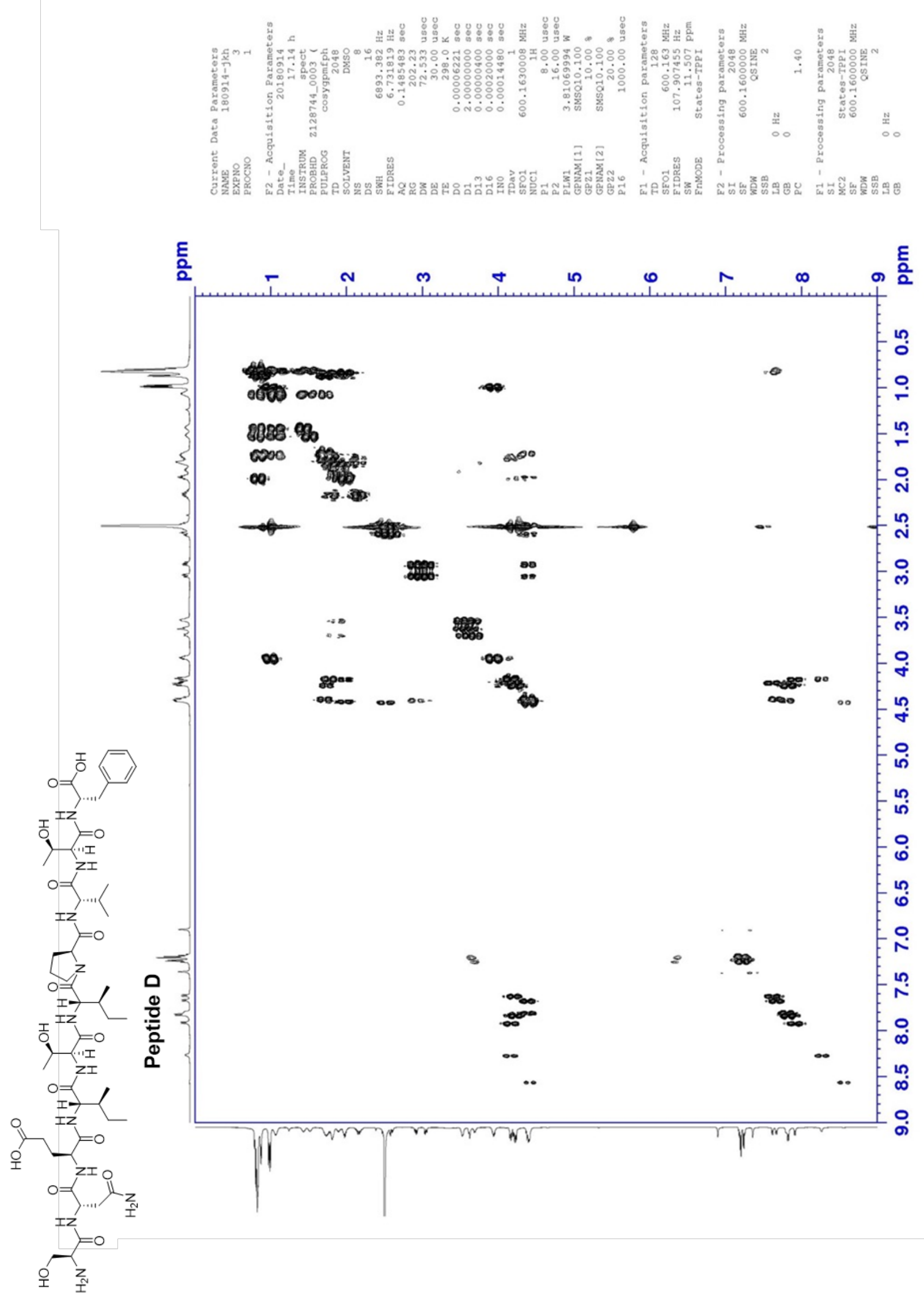




\section{${ }^{1} \mathrm{H}-{ }^{13} \mathrm{C}$ HMBC of Peptide D}

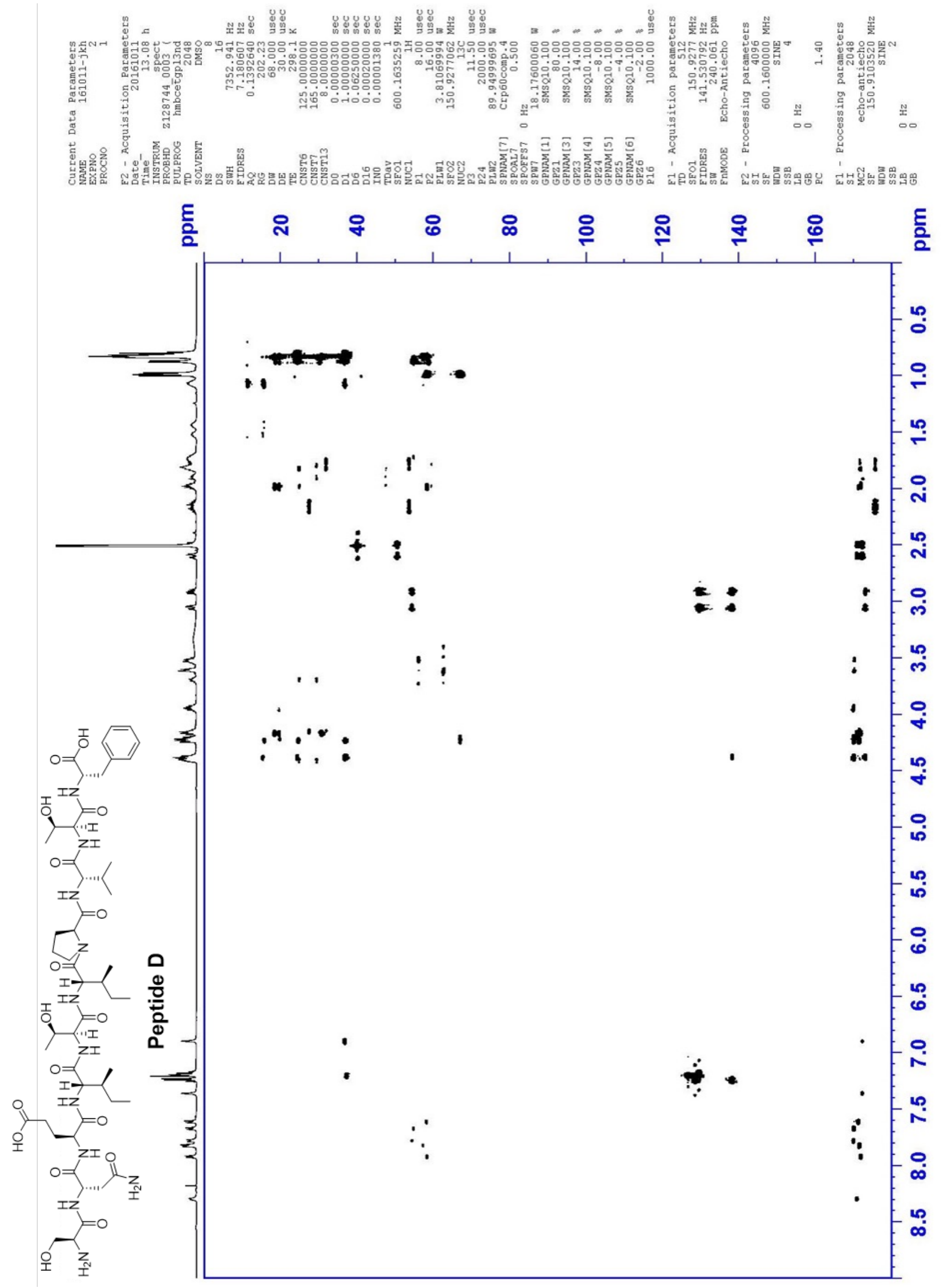




\section{LC/MS of Scrambled Peptide (SP)}

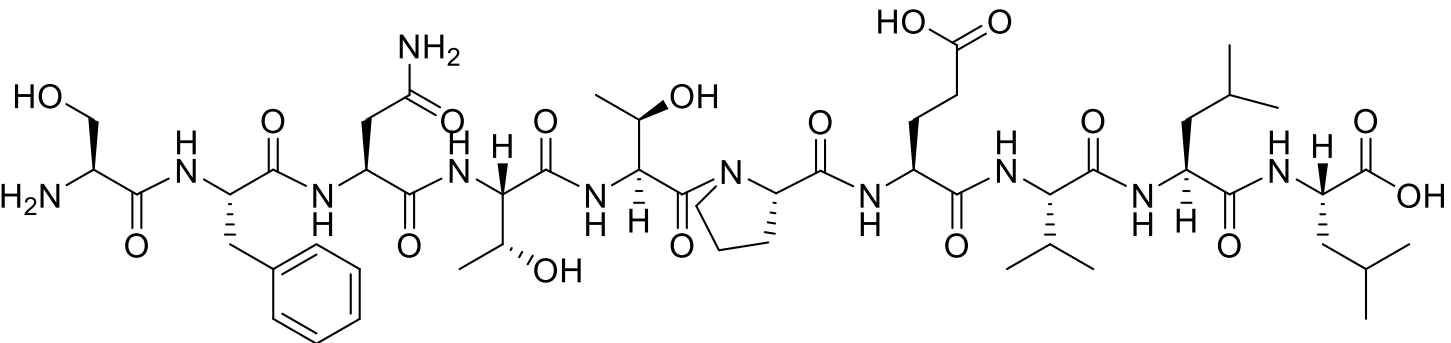

Chemical Formula: $\mathrm{C}_{51} \mathrm{H}_{81} \mathrm{~N}_{11} \mathrm{O}_{17}$

Exact Mass: 1119.58

Molecular Weight: 1120.27

mAU
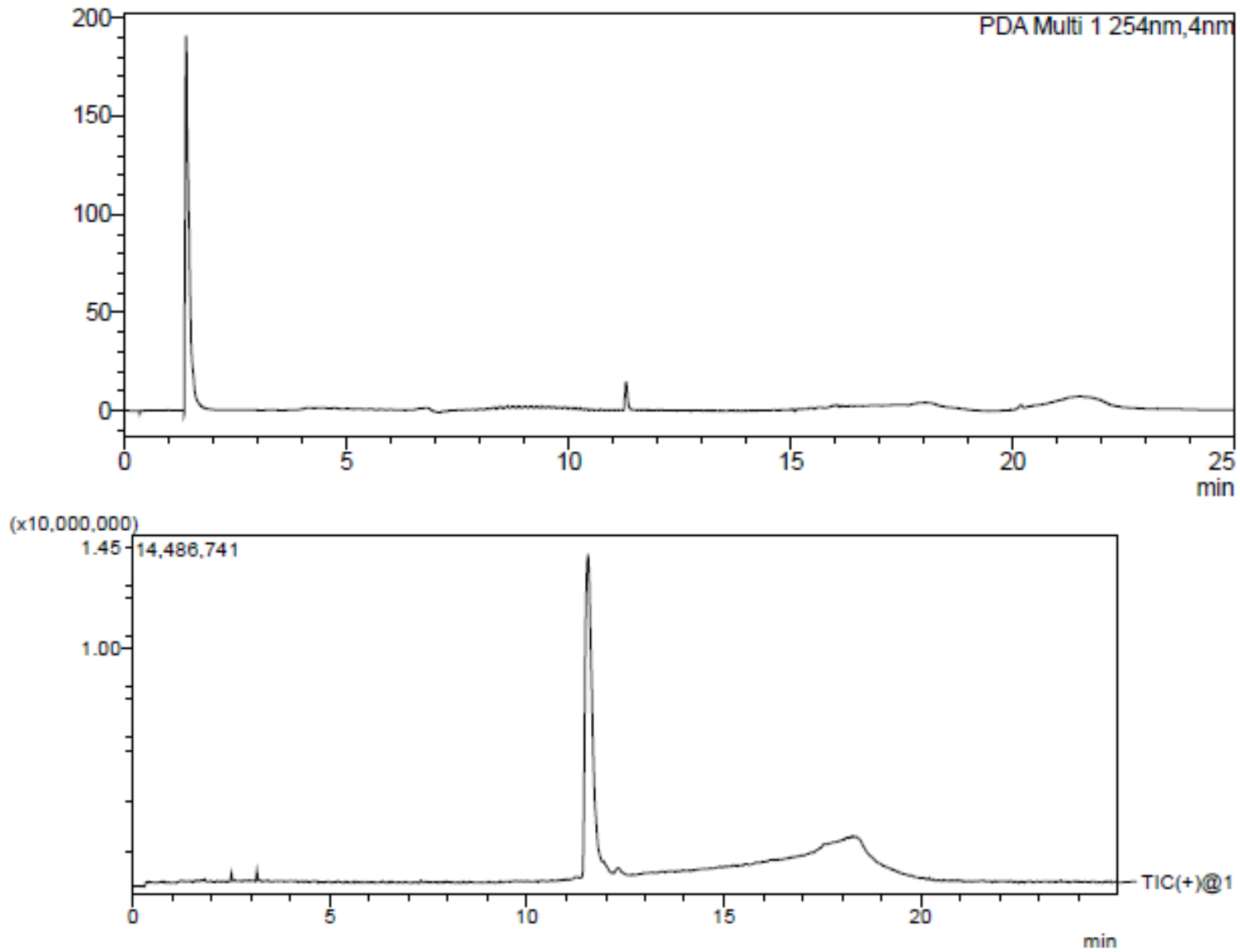

R. Time:11.567(Scan\#.695)

MS Spectrum

MassPeaks: 1909

Segment 1 - Event 1

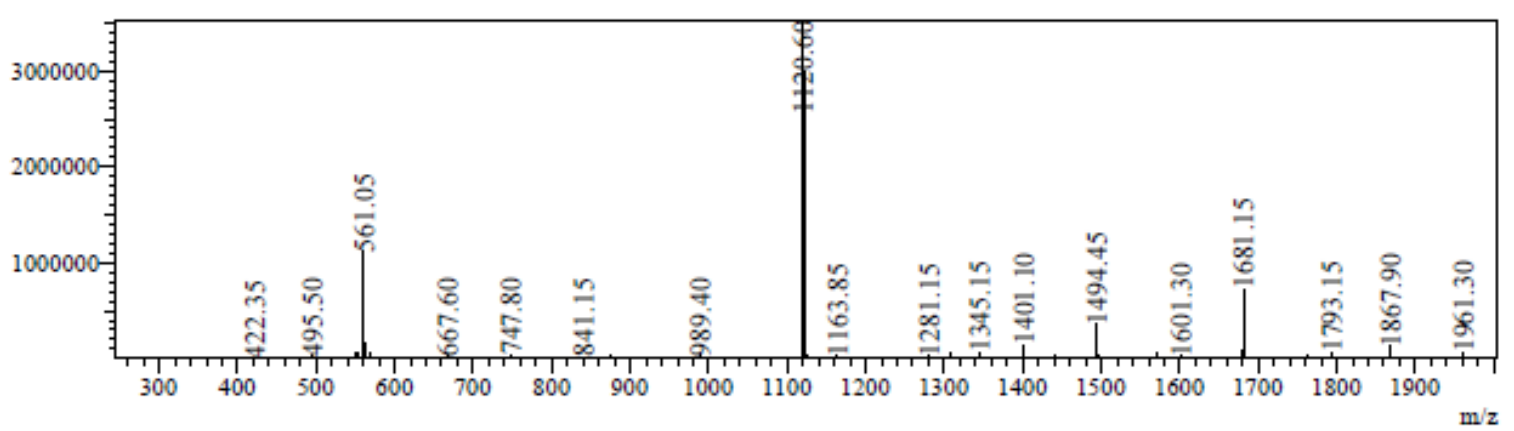




\section{${ }^{1} \mathrm{H}$ NMR of Scrambled Peptide (SP)}
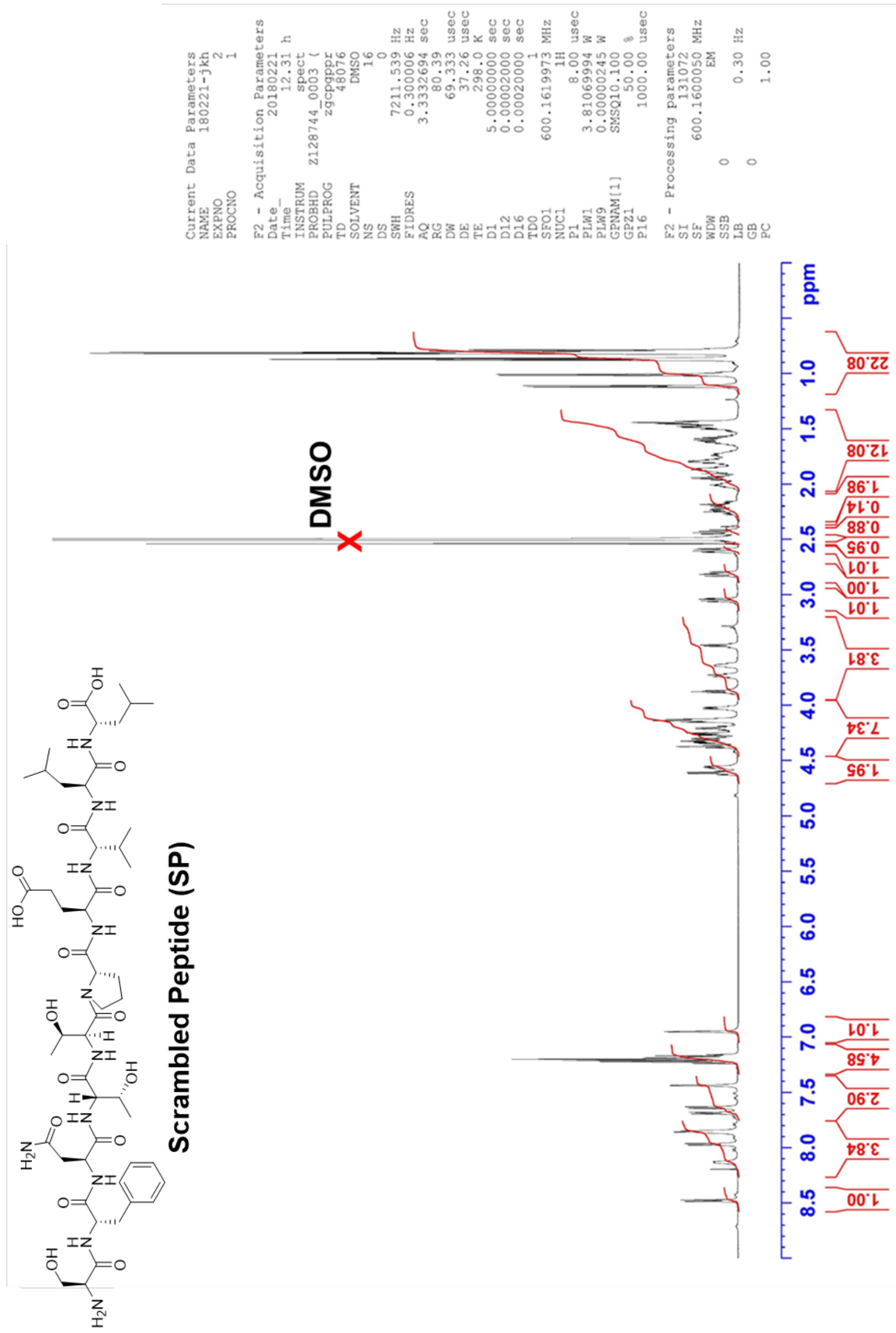


\section{${ }^{1} \mathrm{H}-{ }^{1} \mathrm{H}$ COSY of Scrambled Peptide (SP)}

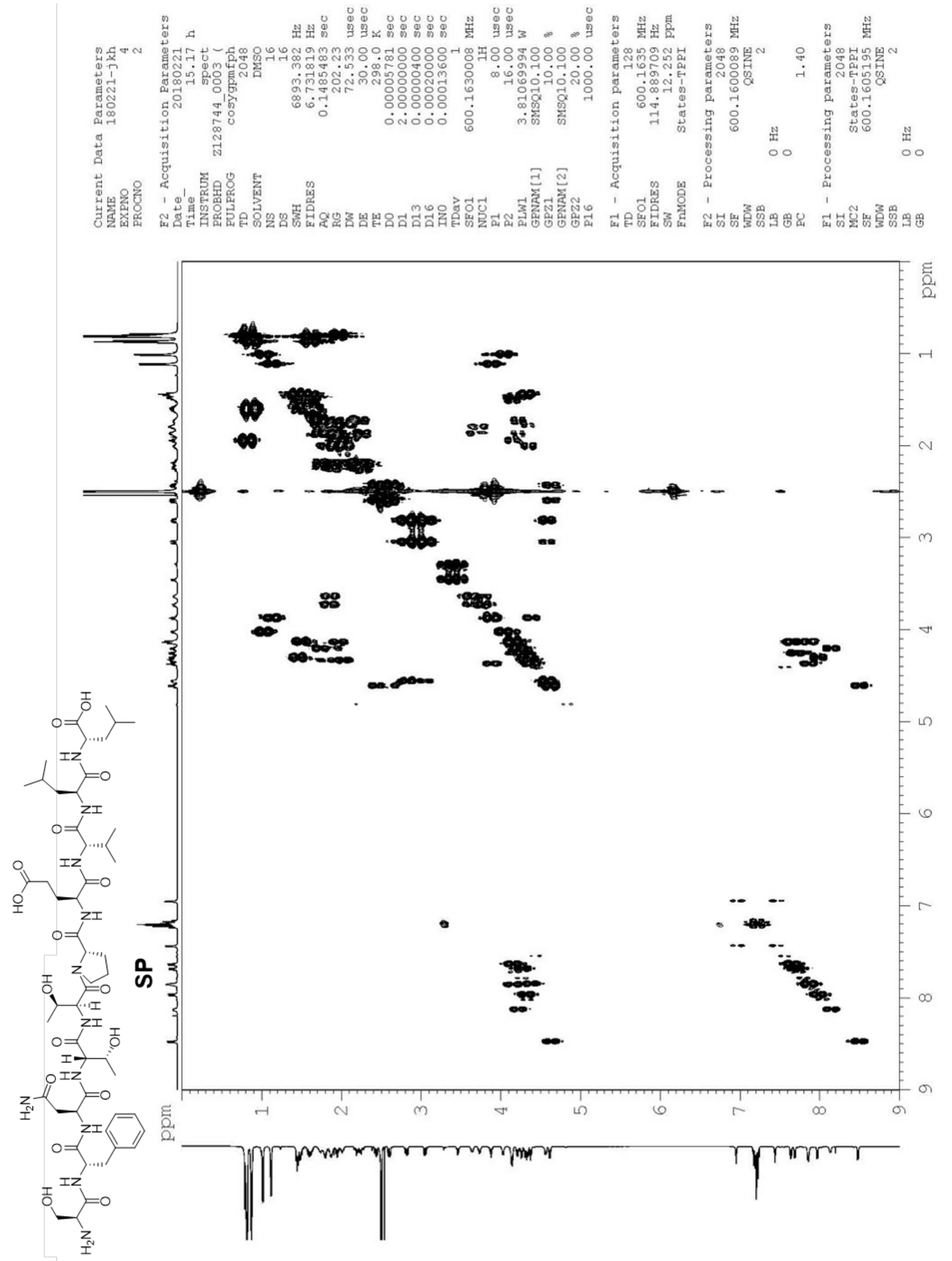




\section{${ }^{1} \mathrm{H}-{ }^{13} \mathrm{C}$ HSQC of Scrambled Peptide (SP)}
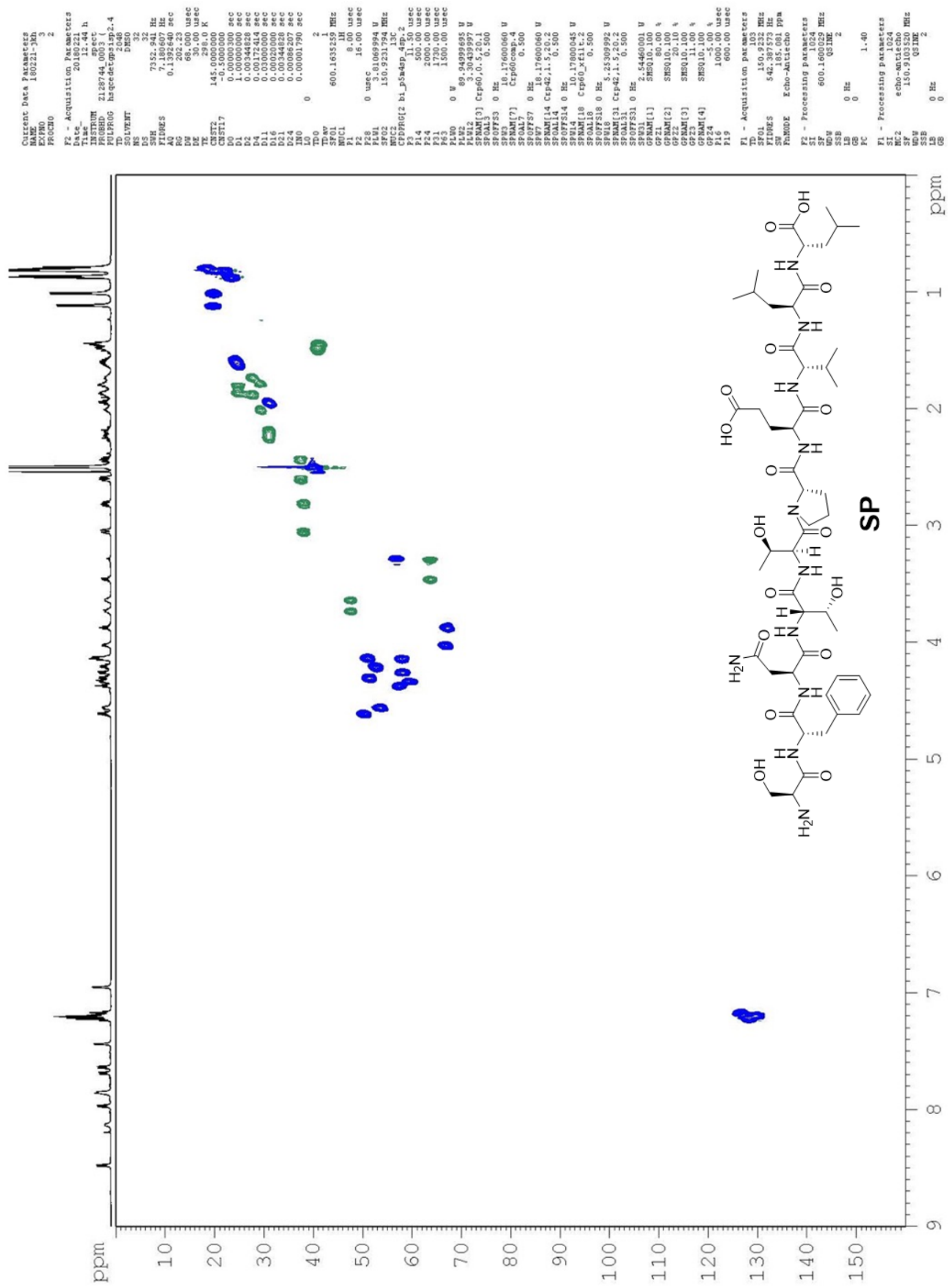


\section{${ }^{1} \mathrm{H}-{ }^{13} \mathrm{C}$ HMBC of Scrambled Peptide (SP)}

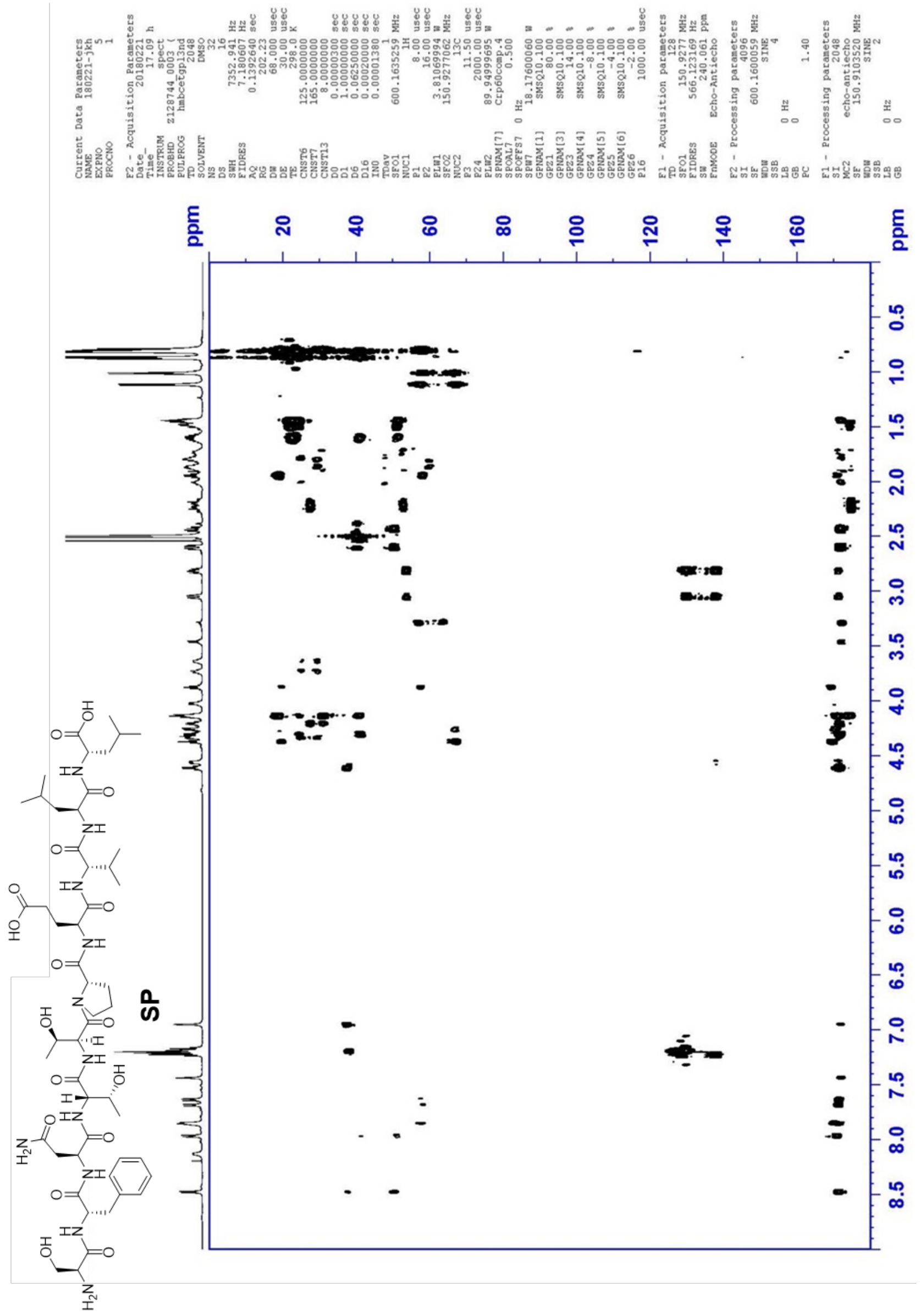




\section{LC/MS of Compound 1}<smiles>N[C@@H](Cc1c[nH]c2ccccc12)C(=O)NC(CC(=O)O)C(=O)N1CCC(C(=O)NC(Cc2ccccc2)C(=O)O)C1=O</smiles>

Chemical Formula: $\mathrm{C}_{29} \mathrm{H}_{33} \mathrm{~N}_{5} \mathrm{O}_{7}$ Exact Mass: 563.24

Molecular Weight: 563.61

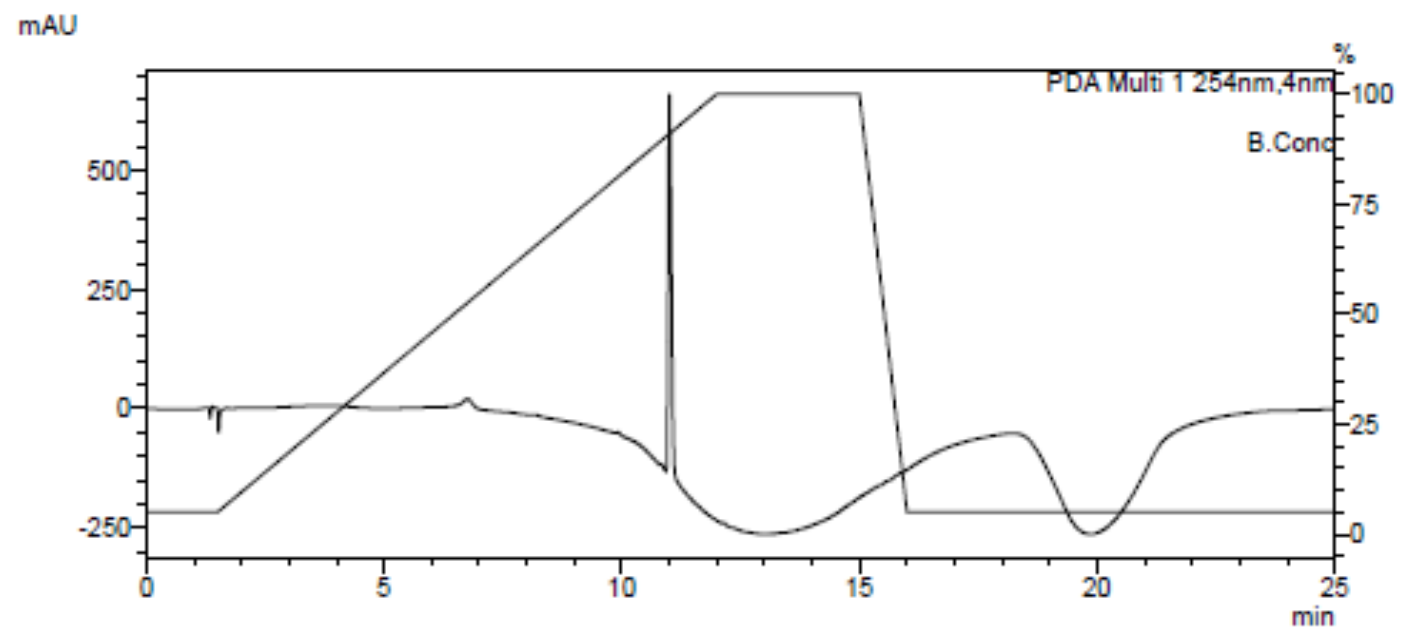

$(x 10,000,000)$

TICI+IQ1

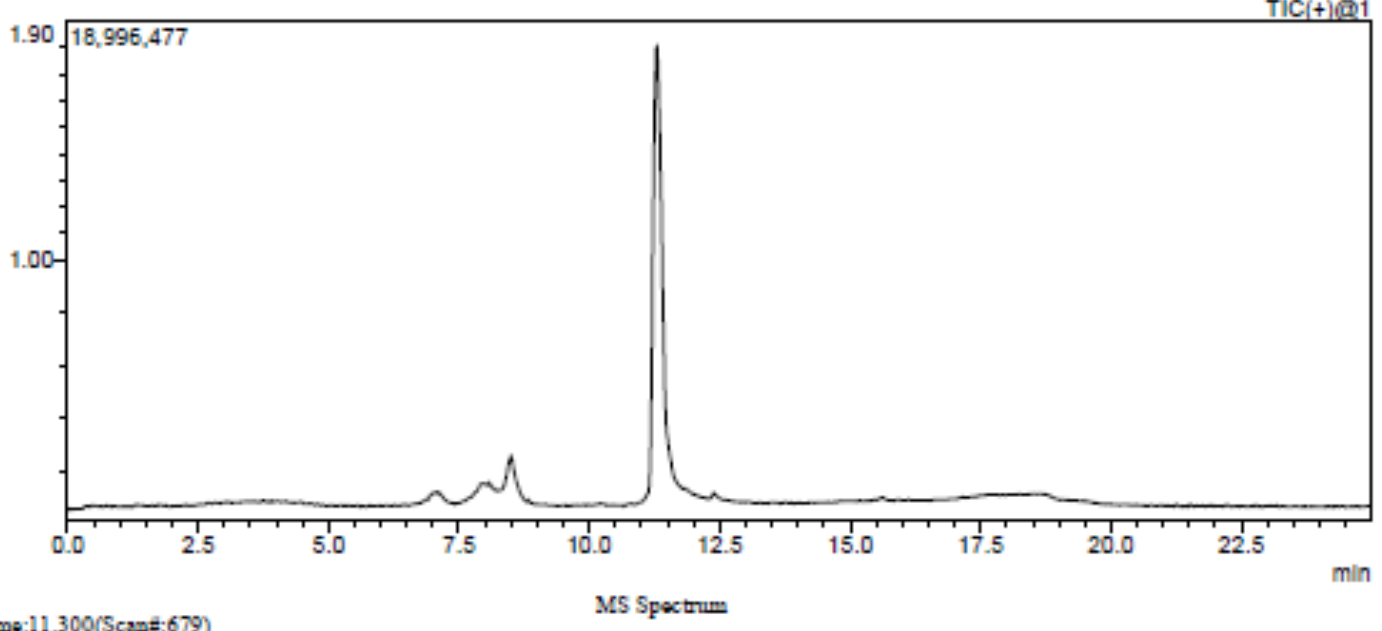

R. Time:11.300(Scune: 679 )

Segmeut 1-Evont 1

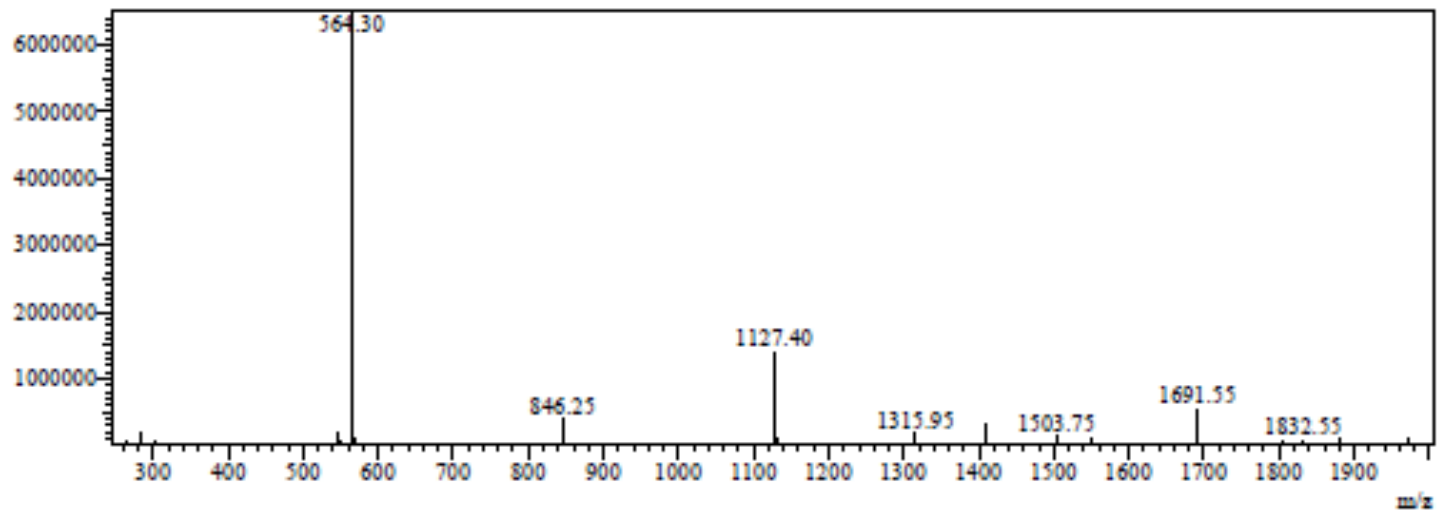




\section{${ }^{1} \mathrm{H}$ NMR of Compound 1}
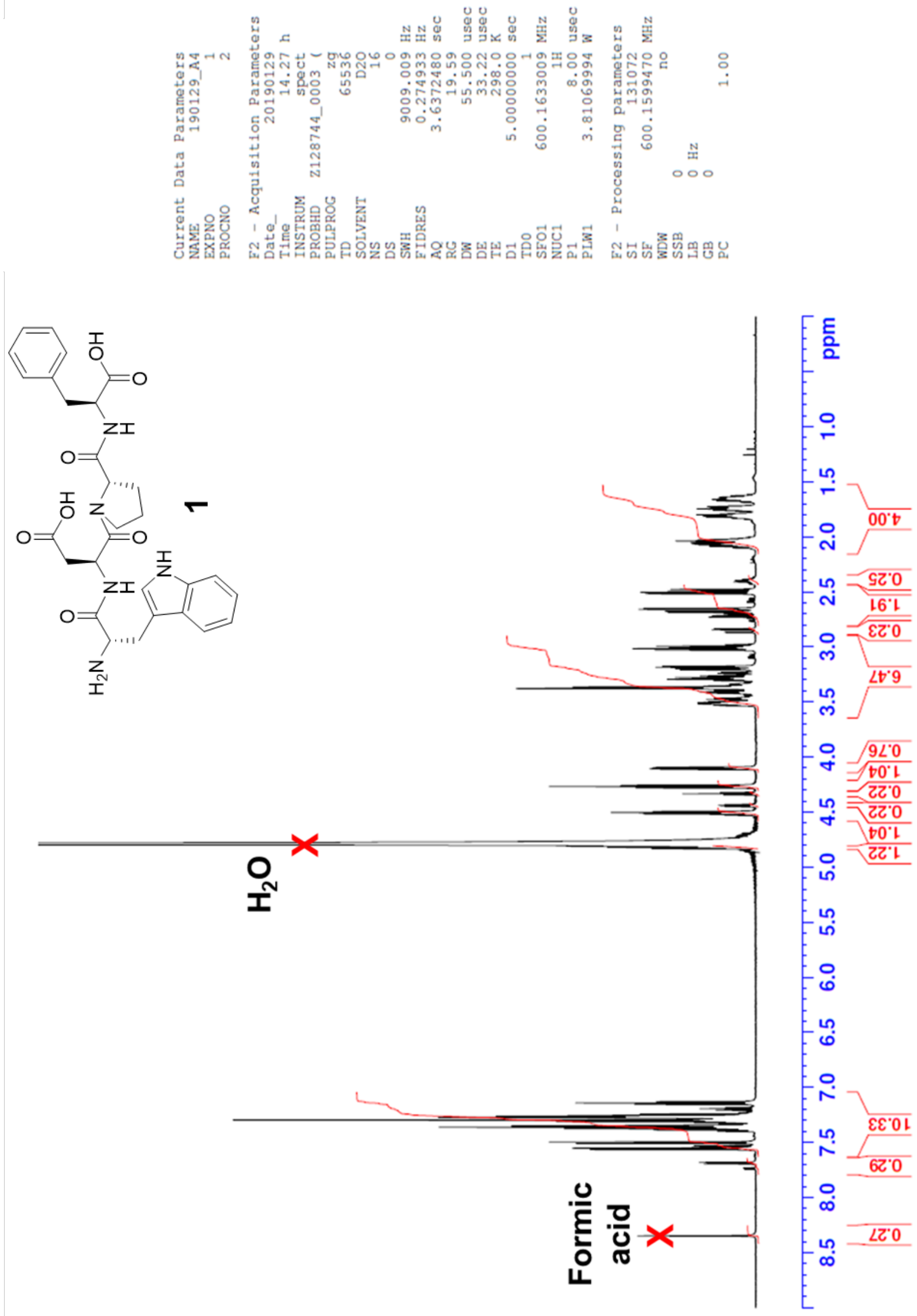


\section{${ }^{1} \mathrm{H}-{ }^{1} \mathrm{H}$ COSY of Compound 1}
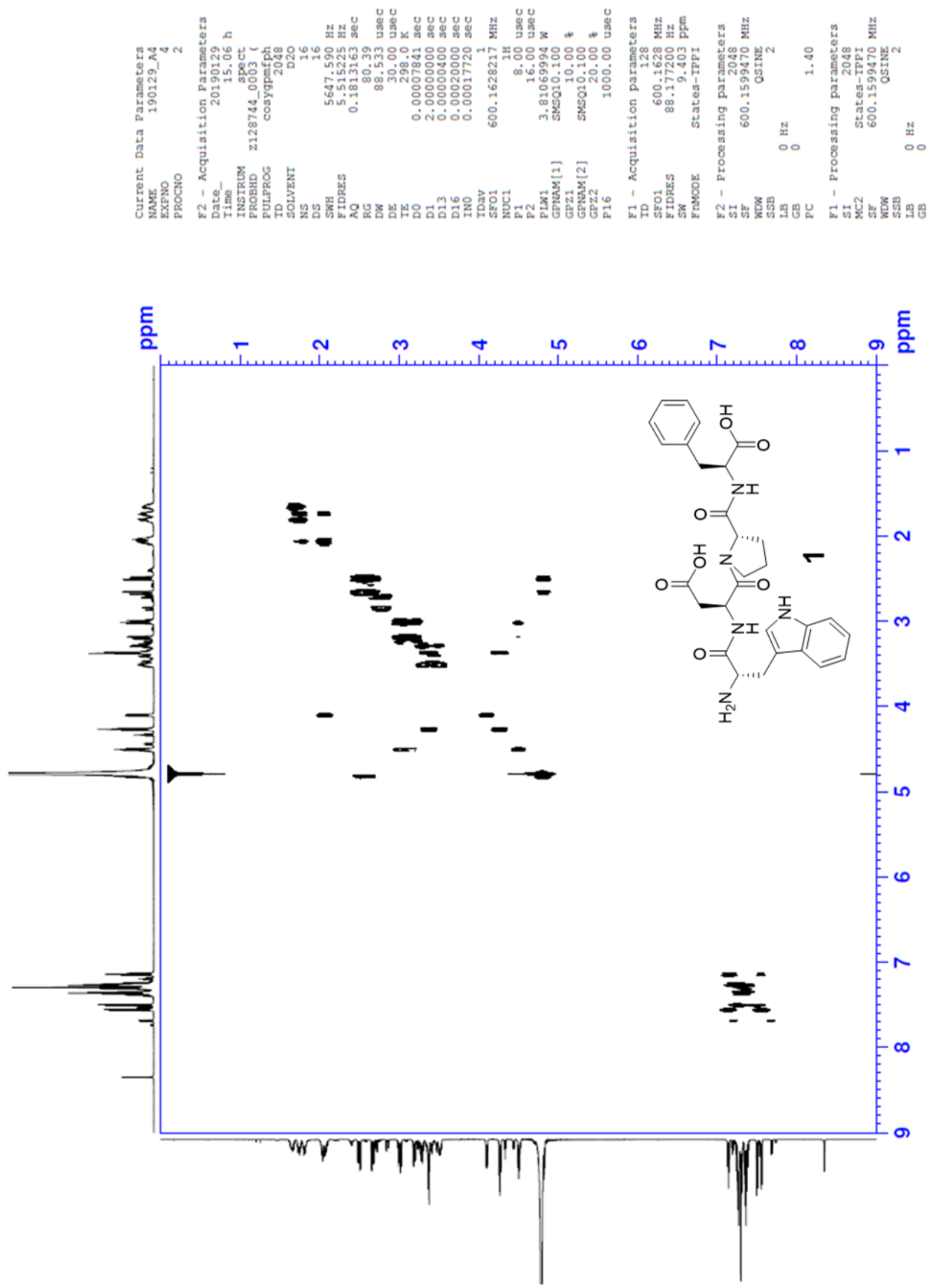


\section{${ }^{1} \mathrm{H}-{ }^{13} \mathrm{C}$ HSQC of Compound 1}

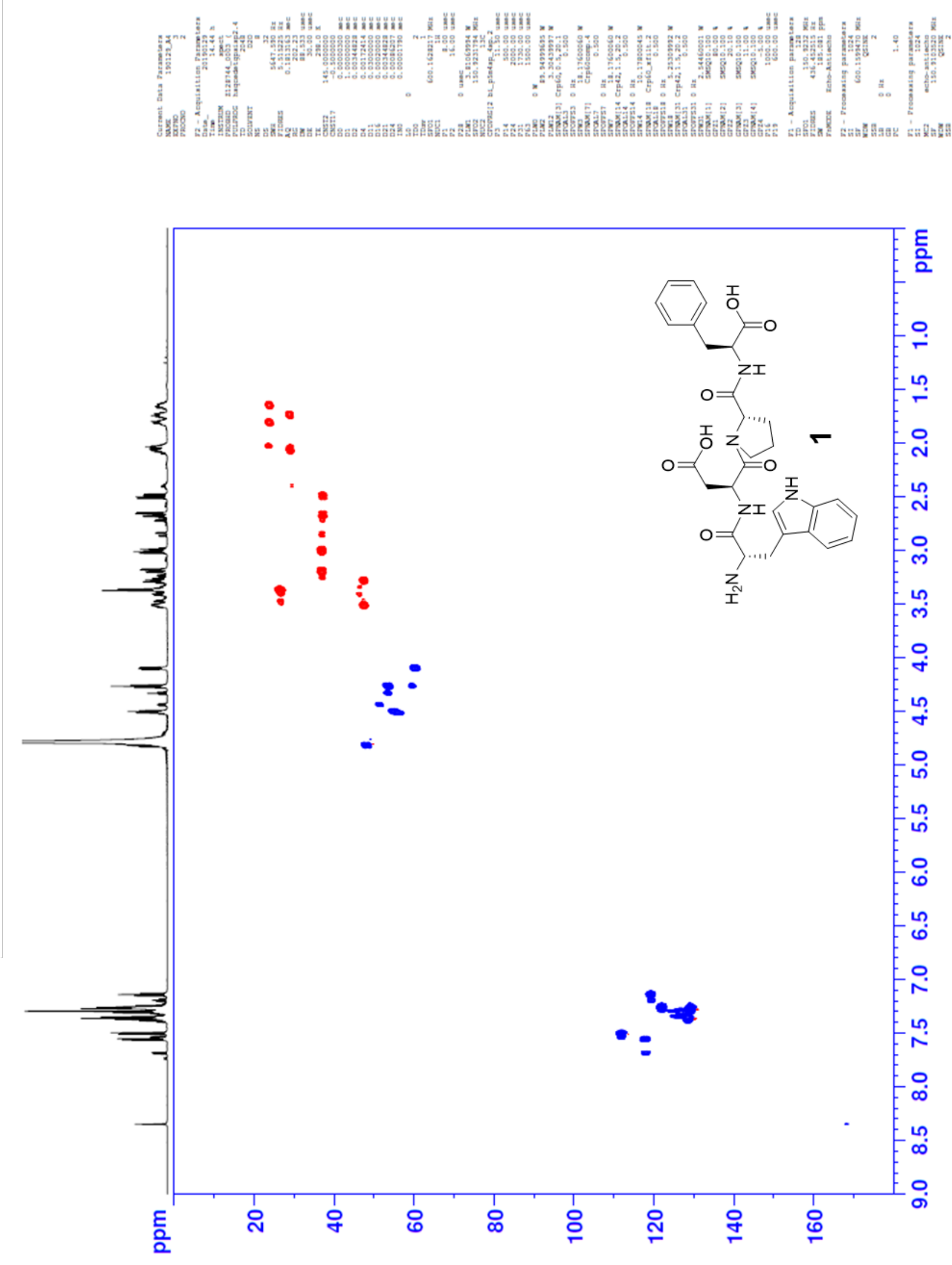




\section{${ }^{1} \mathrm{H}-{ }^{13} \mathrm{C}$ HMBC of Compound 1}
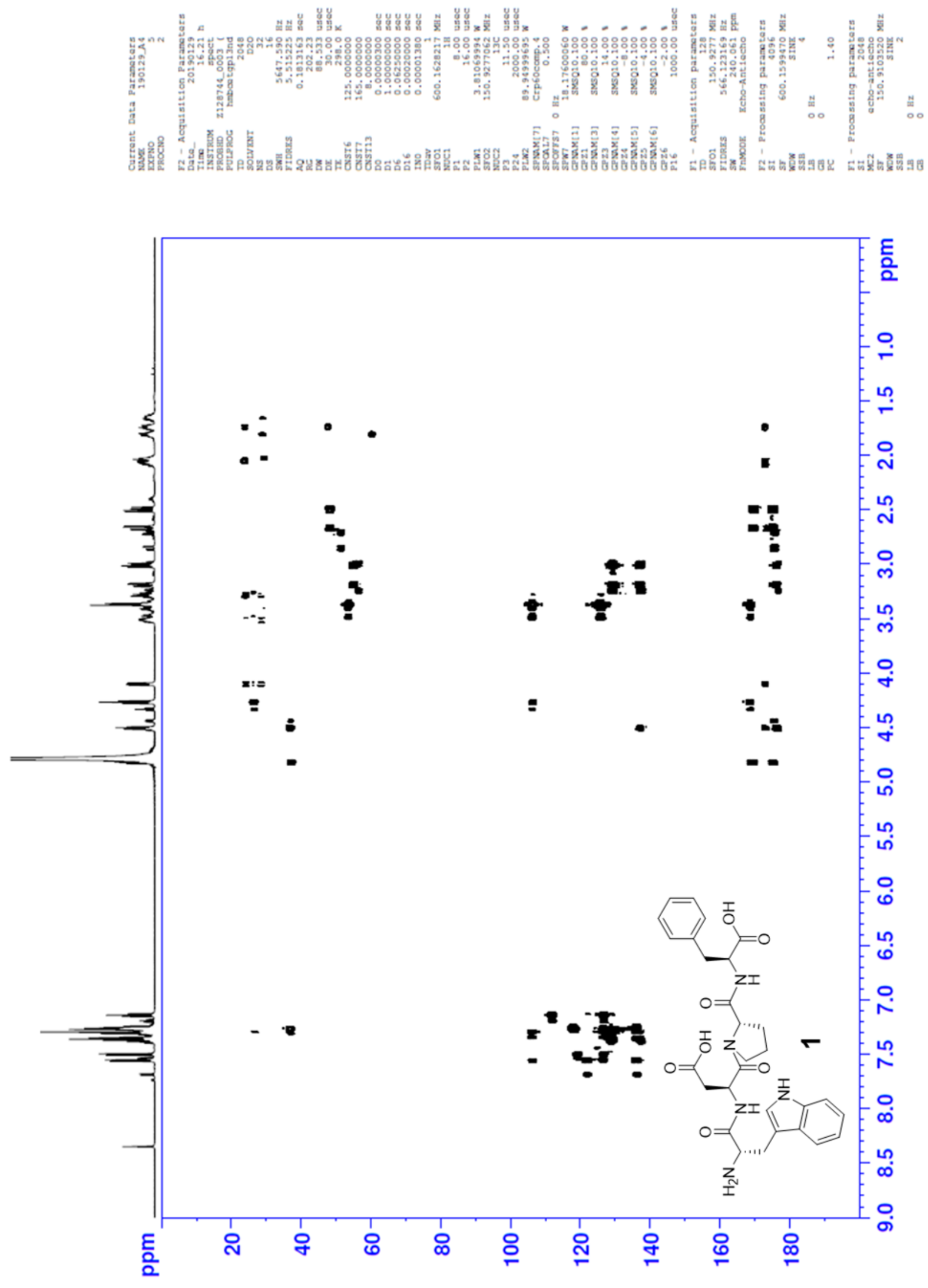


\section{LC/MS of Compound 2}<smiles>N[C@@H](CO)C(=O)N[C@@H](Cc1c[nH]c2ccccc12)C(=O)N[C@@H](CC(=O)O)C(=O)N1CC[C@H](C(=O)N[C@@H](Cc2ccccc2)C(=O)O)C1</smiles>

Chemical Formula: $\mathrm{C}_{32} \mathrm{H}_{38} \mathrm{~N}_{6} \mathrm{O}_{9}$

Exact Mass: 650.27

Molecular Weight: 650.69

mAU

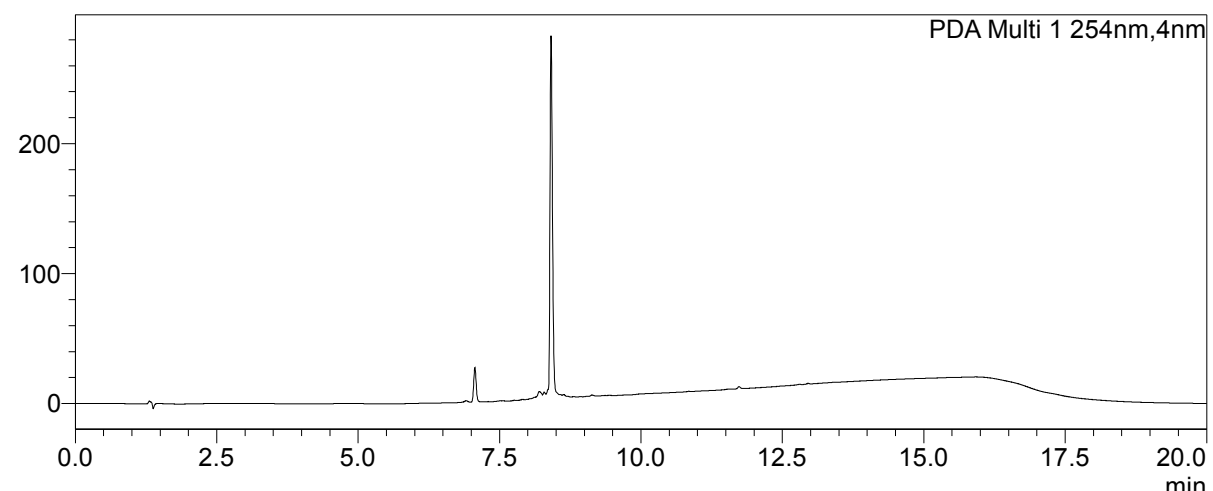

$(\mathrm{x} 1,000,000)$

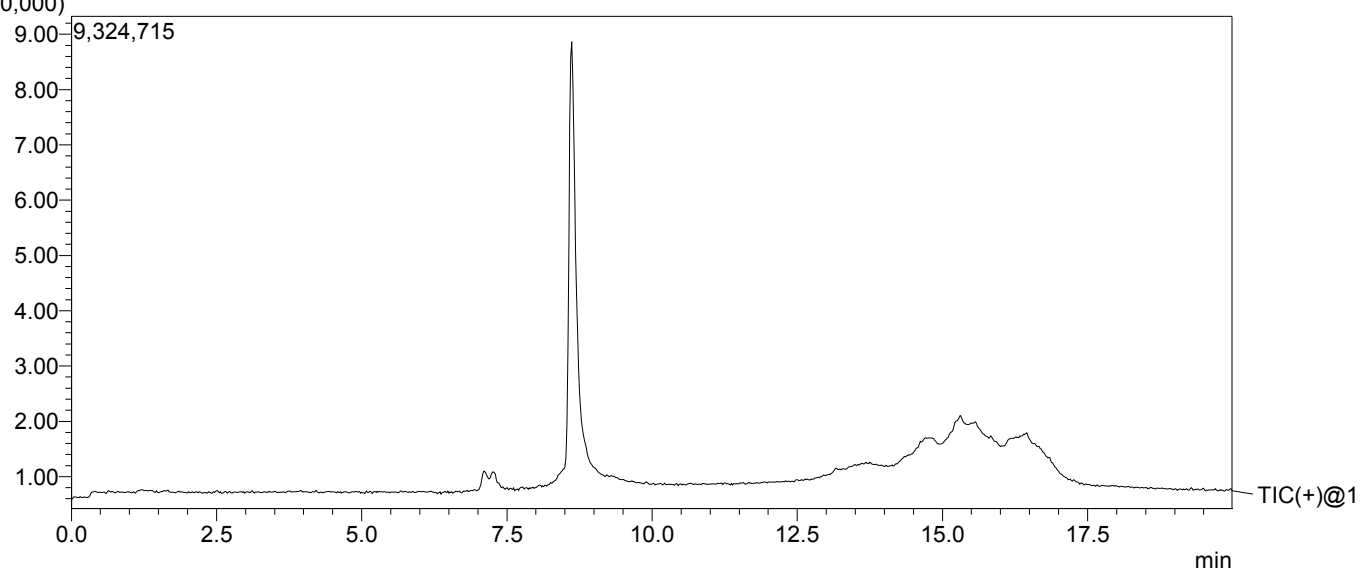

R.Time:8.600(Scan\#:517)

MassPeaks:1866

Segment 1 - Event 1

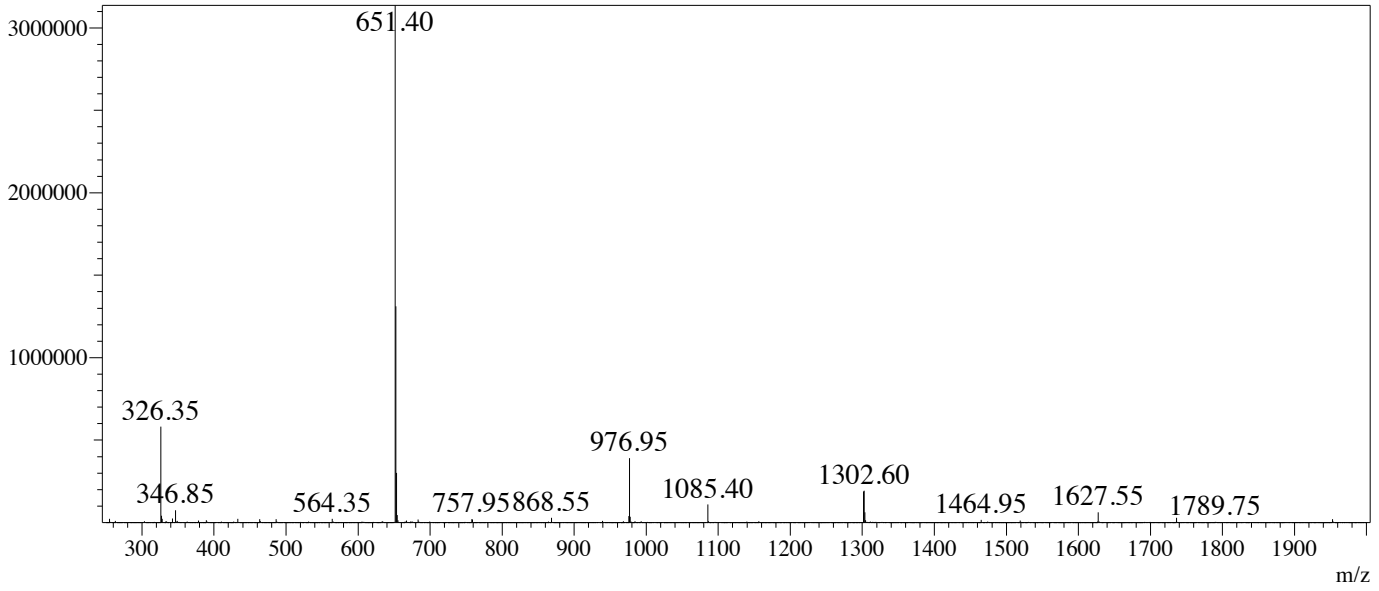




\section{${ }^{1} \mathrm{H}$ NMR of Compound 2}

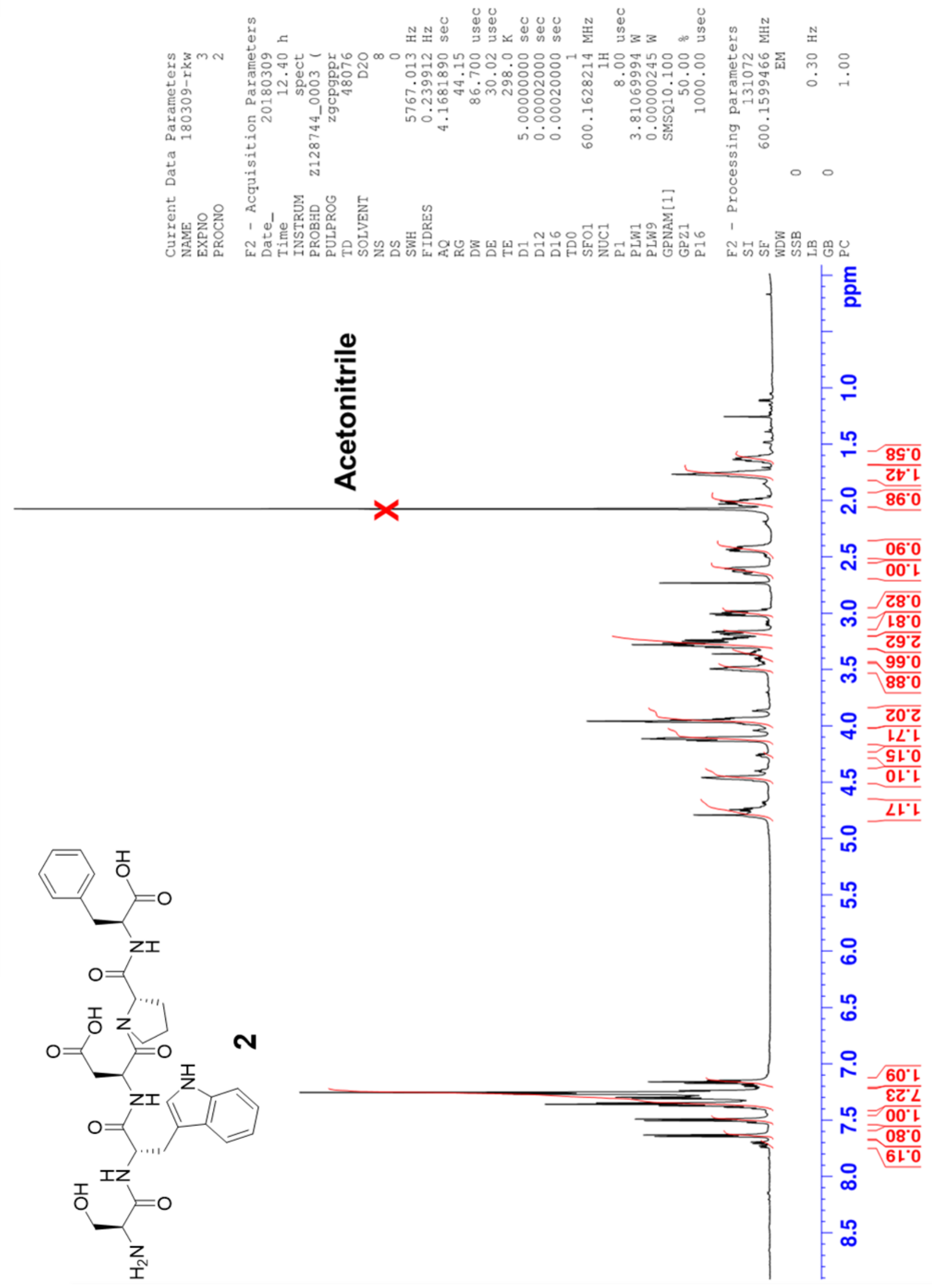




\section{${ }^{1} \mathrm{H}-{ }^{1} \mathrm{H}$ COSY of Compound 2}

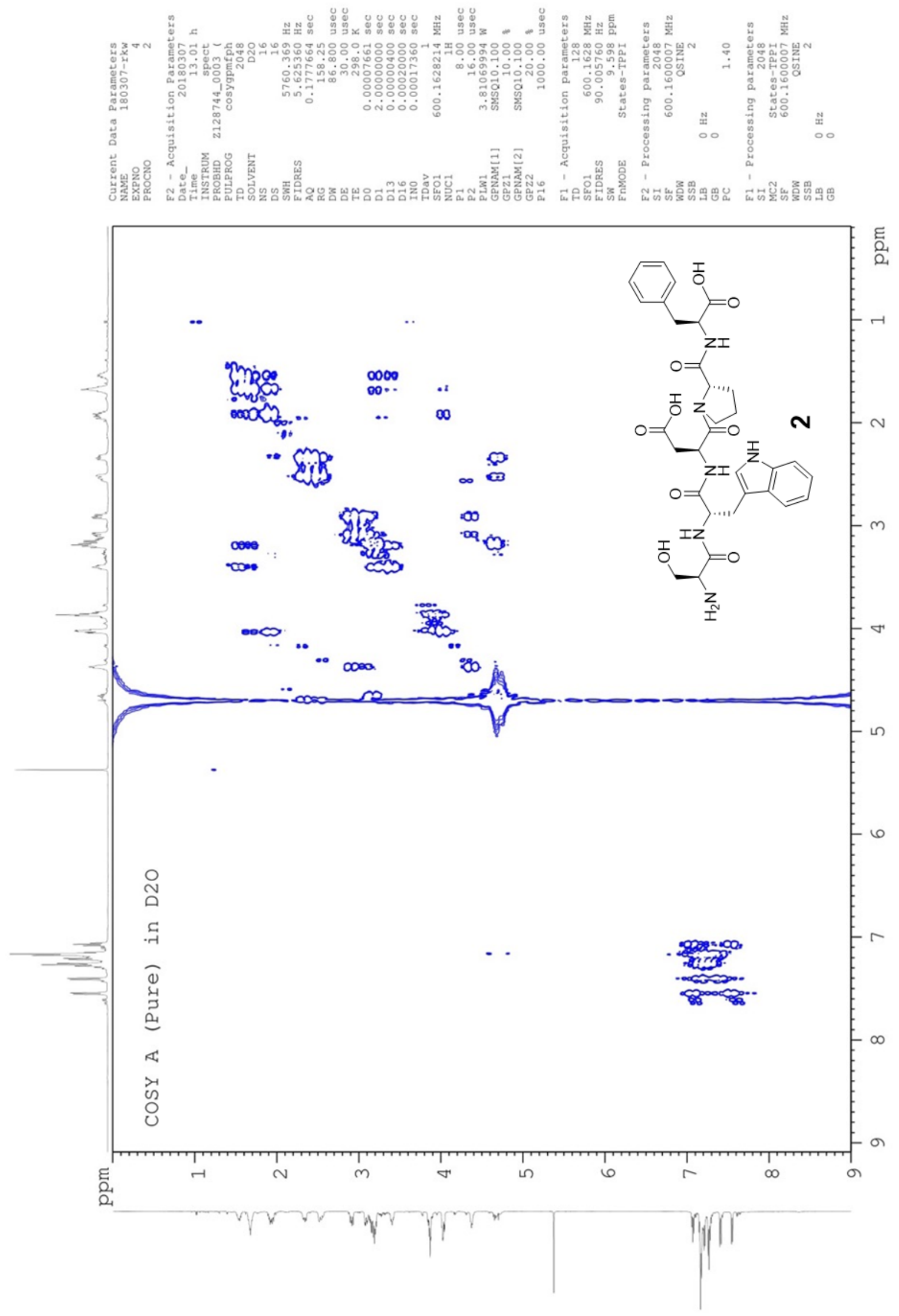




\section{${ }^{1} \mathrm{H}-{ }^{13} \mathrm{C}$ HSQC of Compound 2}
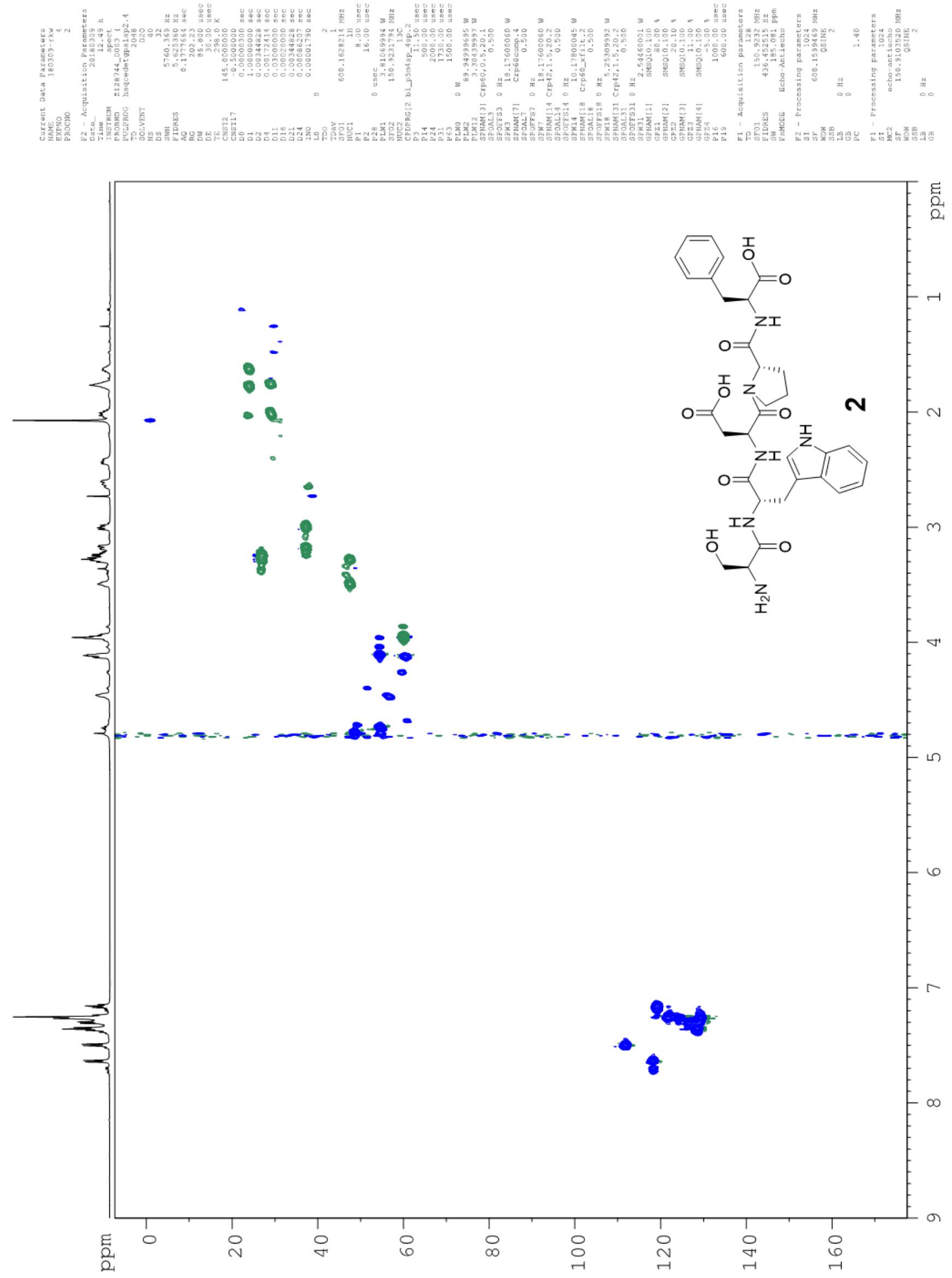


\section{${ }^{1} \mathrm{H}-{ }^{13} \mathrm{C}$ HMBC of Compound 2}

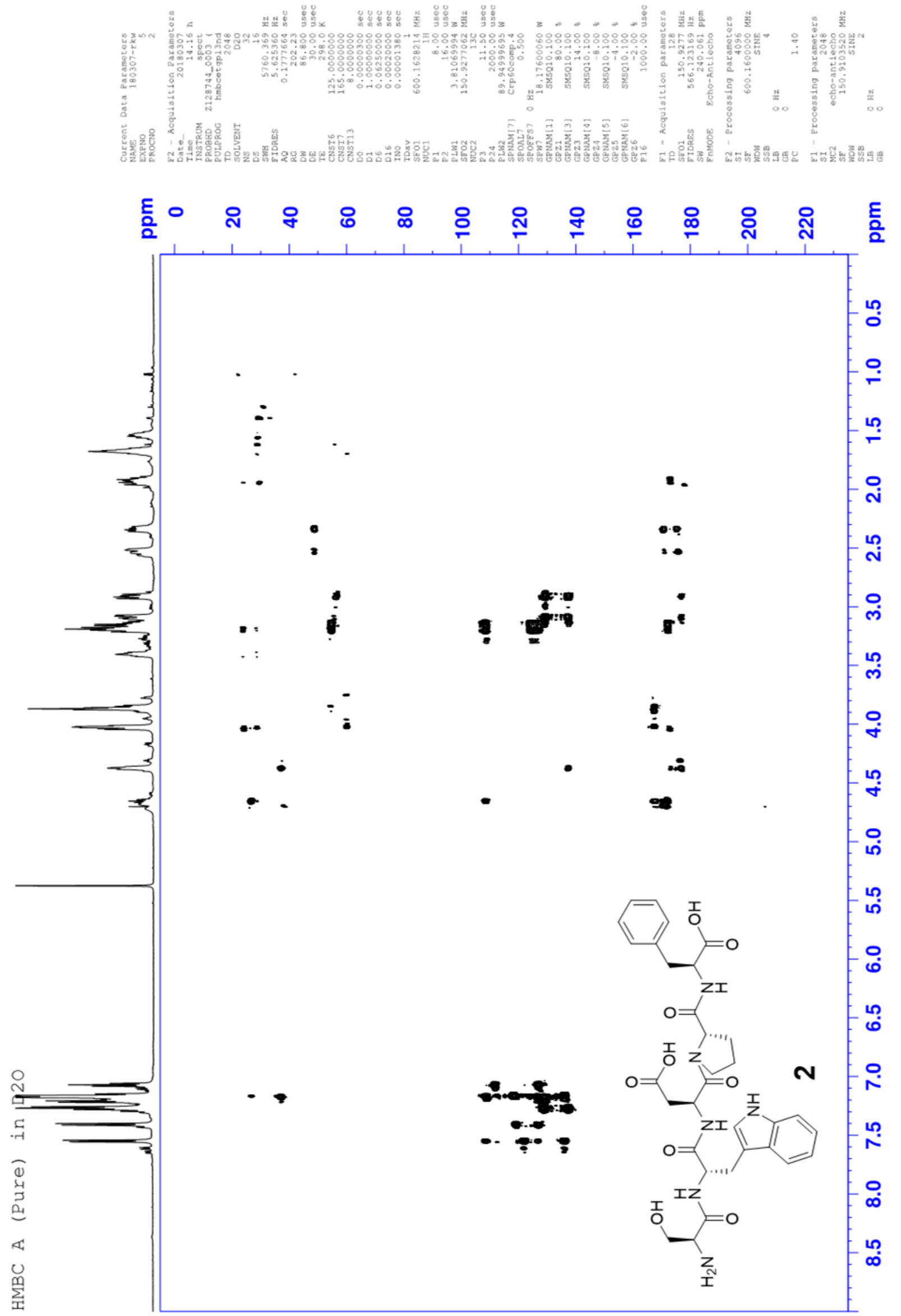




\section{LC/MS of Compound 3}

$\mathrm{H}_{2} \mathrm{~N}$<smiles>CC(Cc1c[nH]c2ccccc12)NC(=O)C[C@H](CC(=O)O)C(=O)N1CC[C@H](C(=O)I)N1</smiles>

mAU

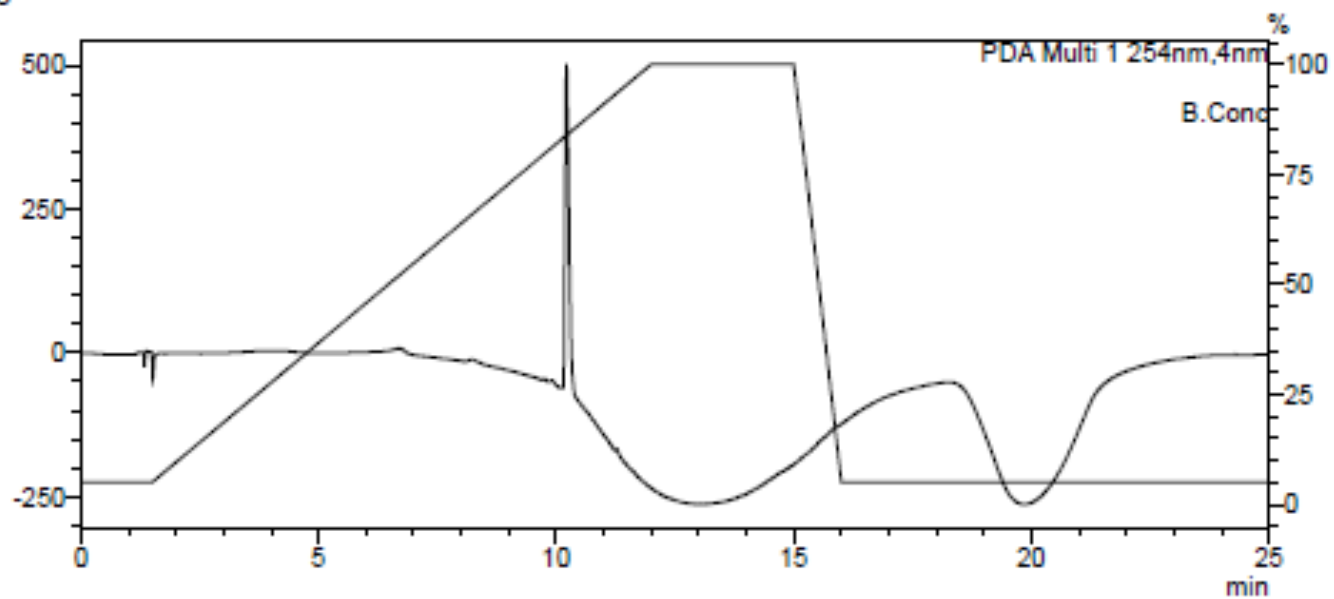<smiles>N=C(N)NCCC[C@H](NC(=O)C(N)Cc1ccccc1)C(=O)O</smiles>

Chemical Formula: $\mathrm{C}_{35} \mathrm{H}_{45} \mathrm{~N}_{9} \mathrm{O}_{8}$

Exact Mass: 719.34

Molecular Weight: 719.80

$(x 10,000,000)$

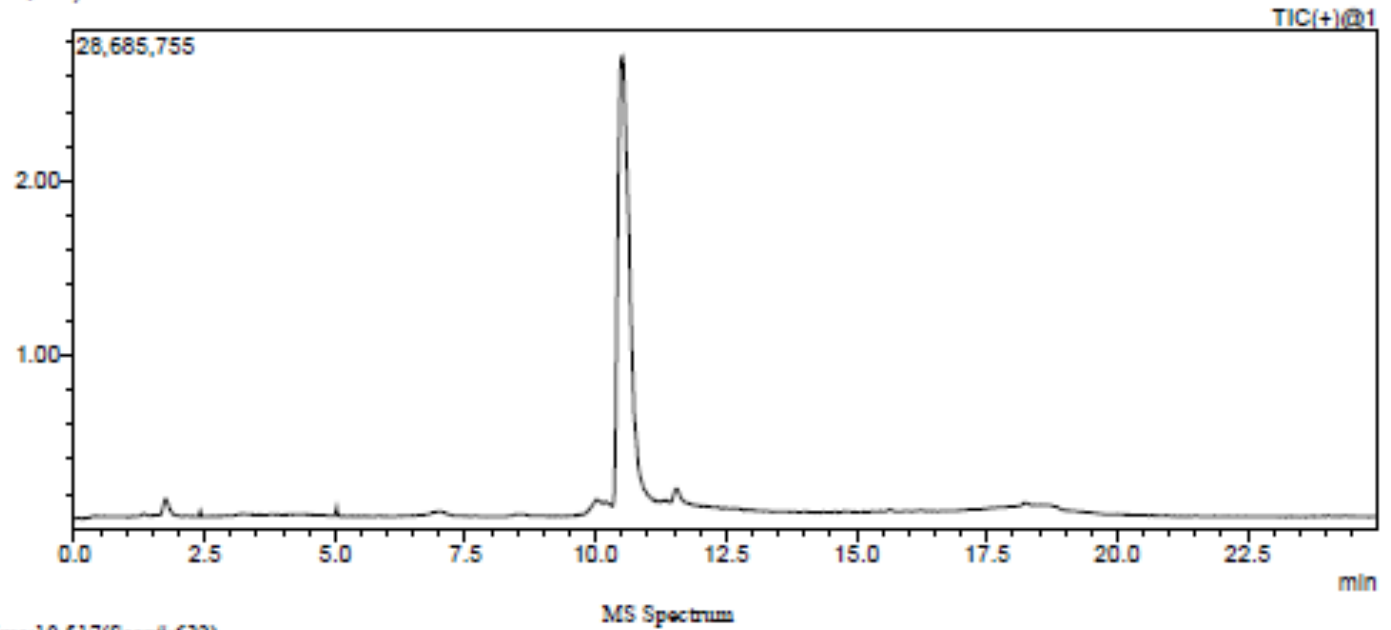

R. Time:10.517(Scan=-632)

MS Spectrum

$$
\text { Sogmear } 1 \text {-Evont } 1
$$

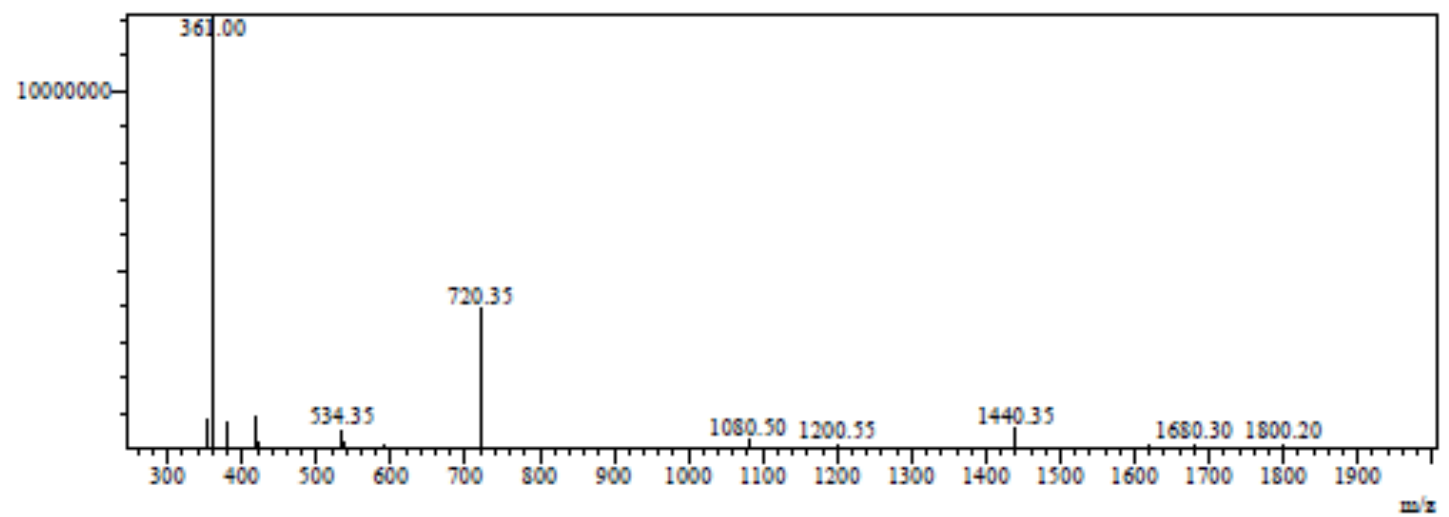




\section{${ }^{1} \mathrm{H}$ NMR of Compound 3}
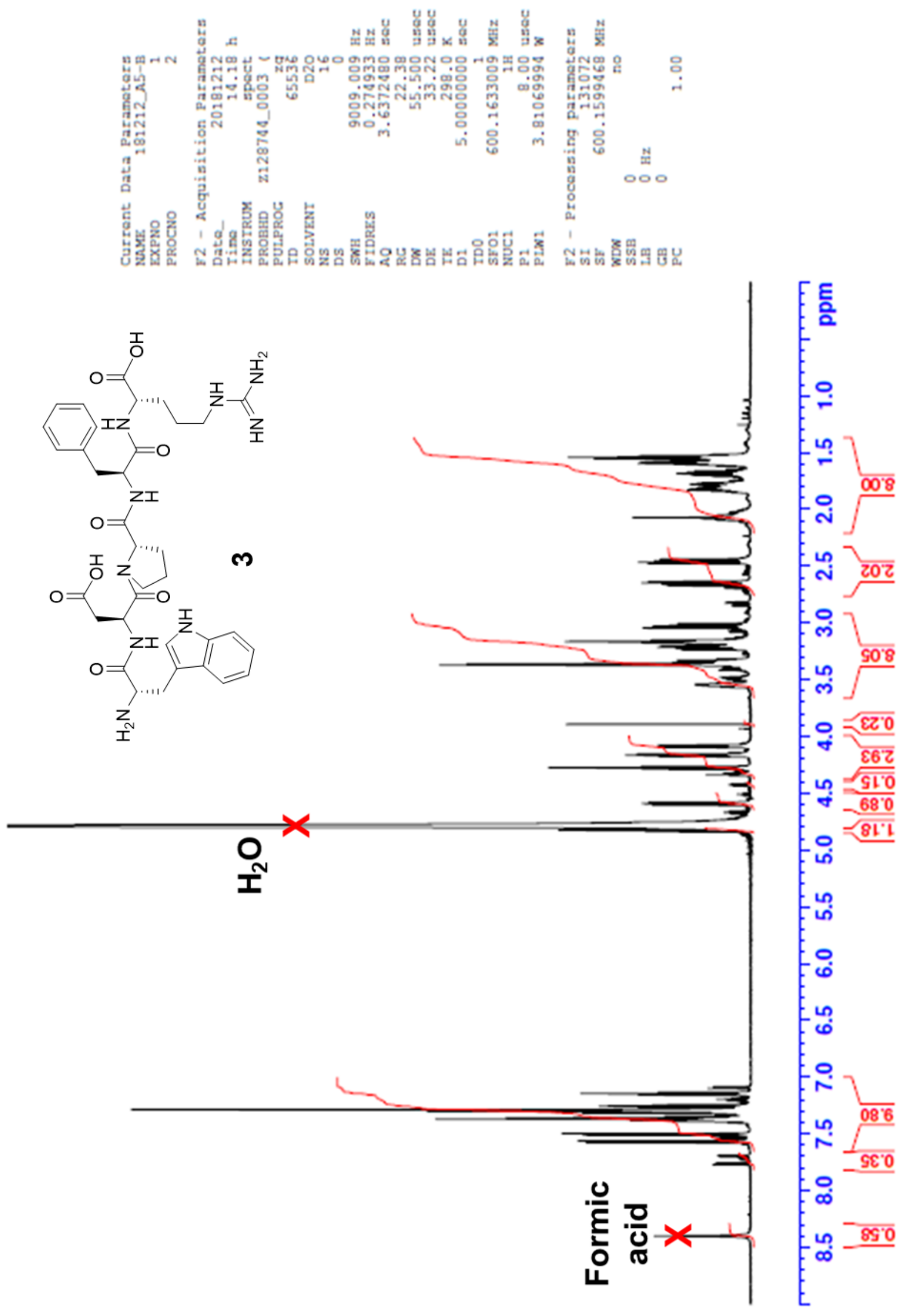


\section{${ }^{1} \mathrm{H}-{ }^{1} \mathrm{H}$ COSY of Compound 3}
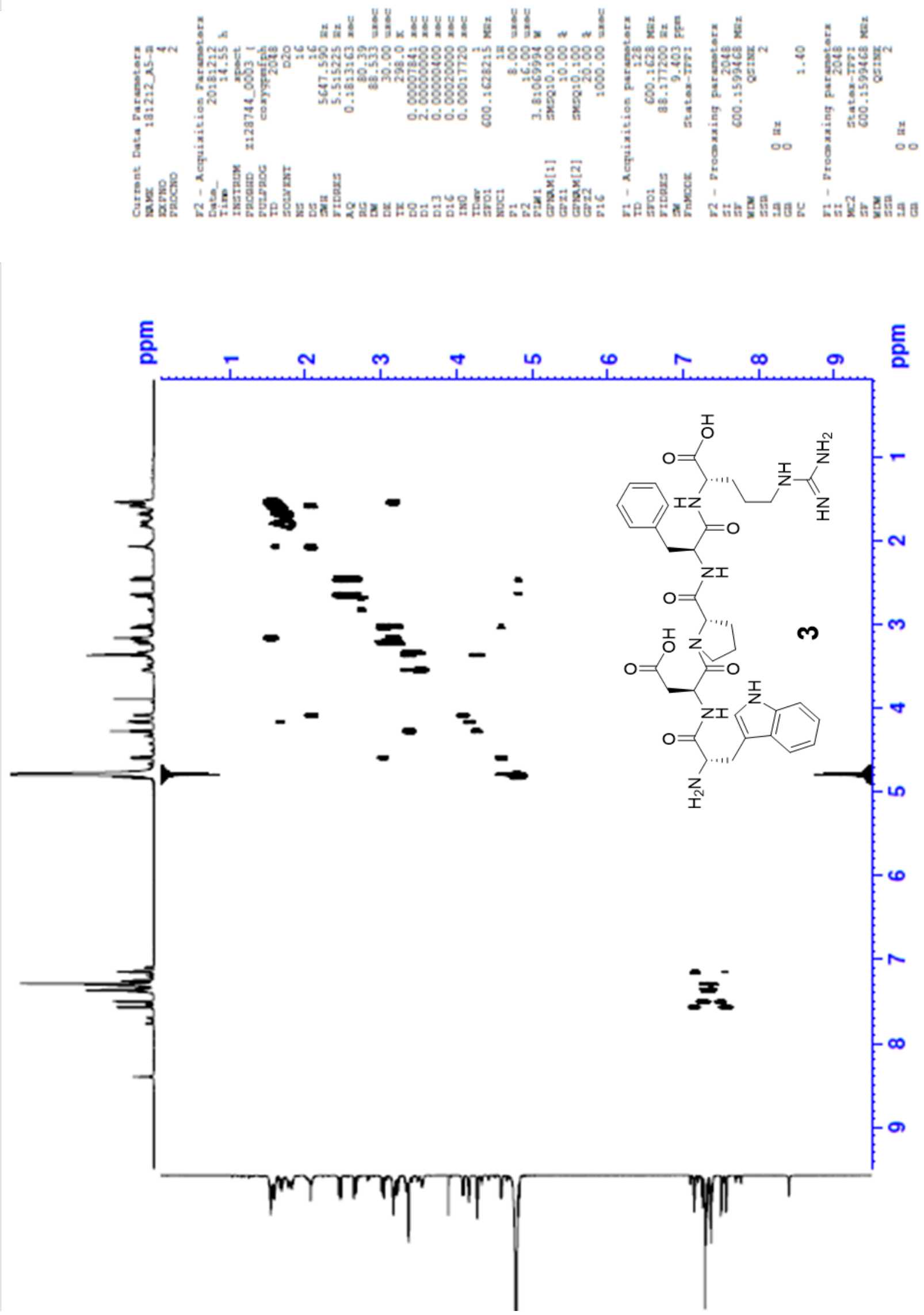


\section{${ }^{1} \mathrm{H}-{ }^{13} \mathrm{C}$ HSQC of Compound 3}
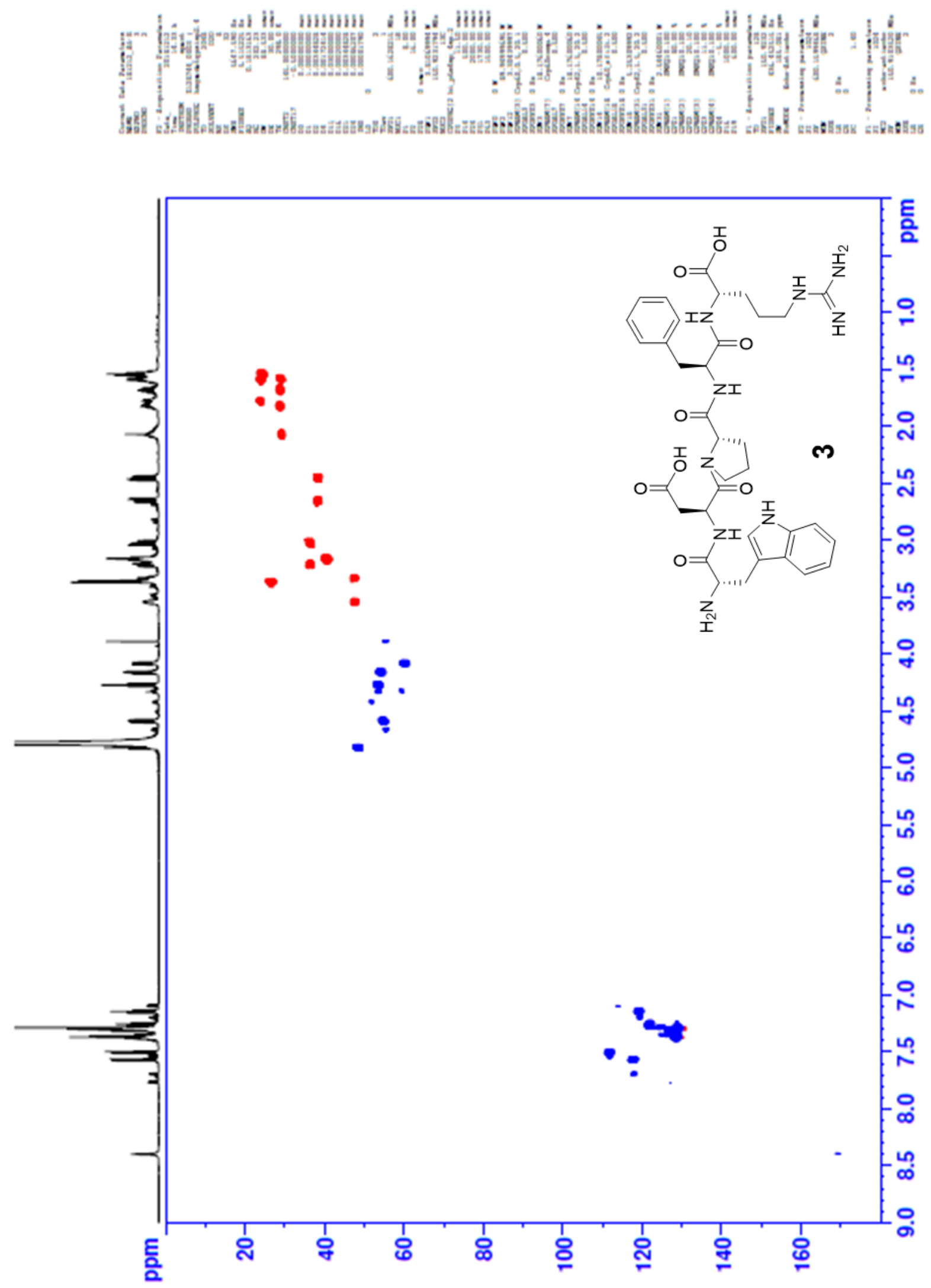


\section{${ }^{1} \mathrm{H}-{ }^{13} \mathrm{C}$ HMBC of Compound 3}
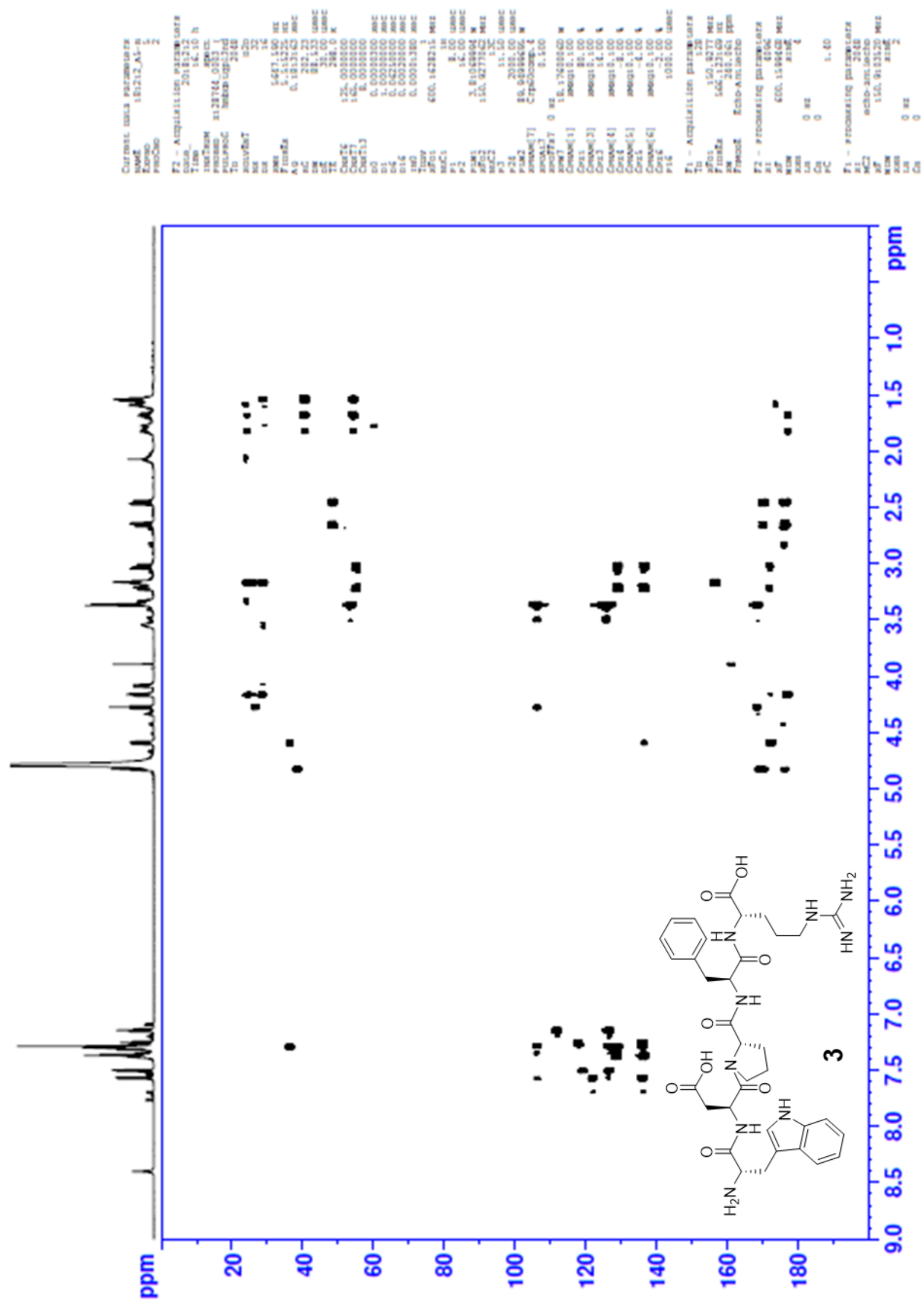


\section{LC/MS of Compound 4}<smiles>C[C@H](N)C(=O)N[C@@H](Cc1c[nH]c2ccccc12)C(=O)N[C@@H](CC(=O)O)C(=O)N1CCC[C@H]1C(=O)N[C@@H](Cc1ccccc1)C(=O)O</smiles>

Chemical Formula: $\mathrm{C}_{32} \mathrm{H}_{38} \mathrm{~N}_{6} \mathrm{O}_{8}$

Exact Mass: 634.28

Molecular Weight: 634.69

mAU

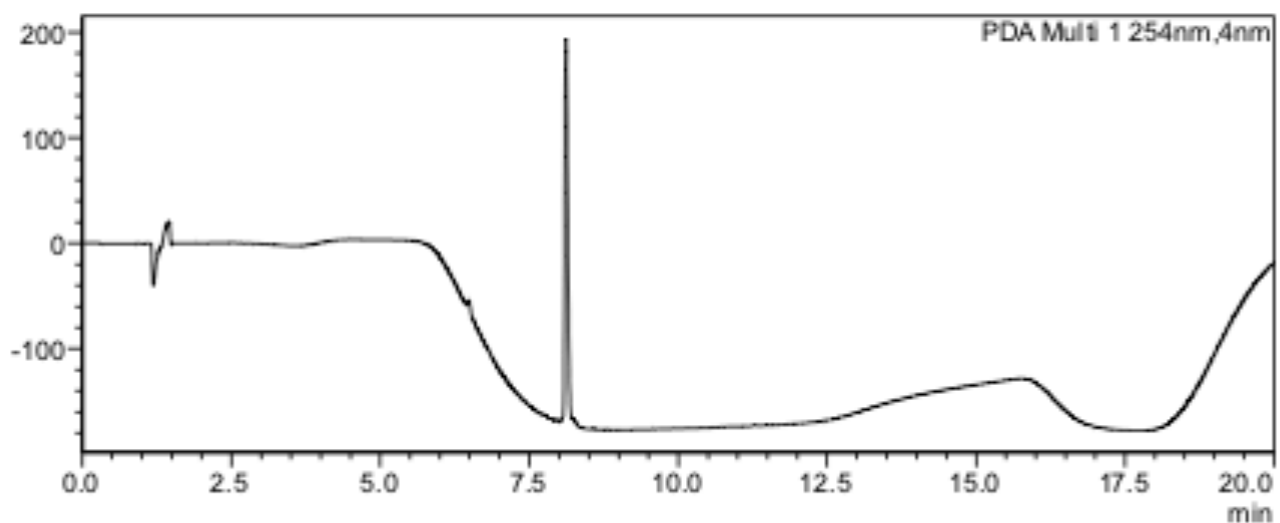

$(x 1,000,000)$

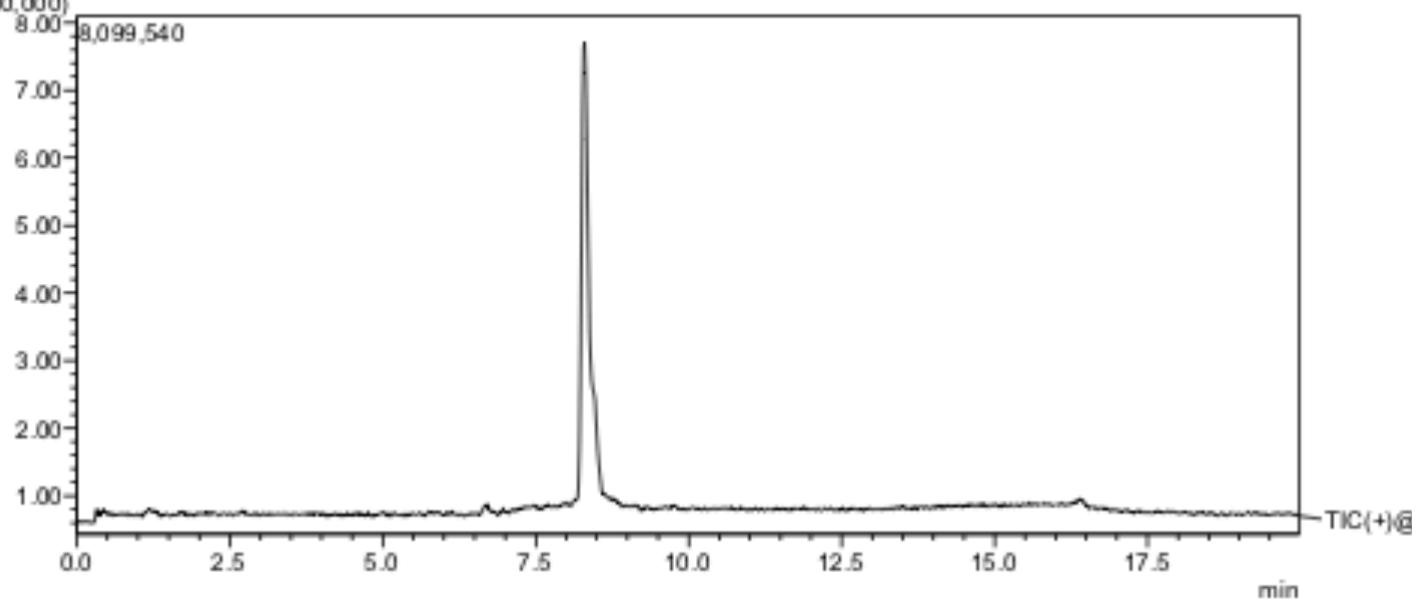

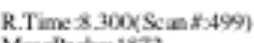

Masspedis: 1872

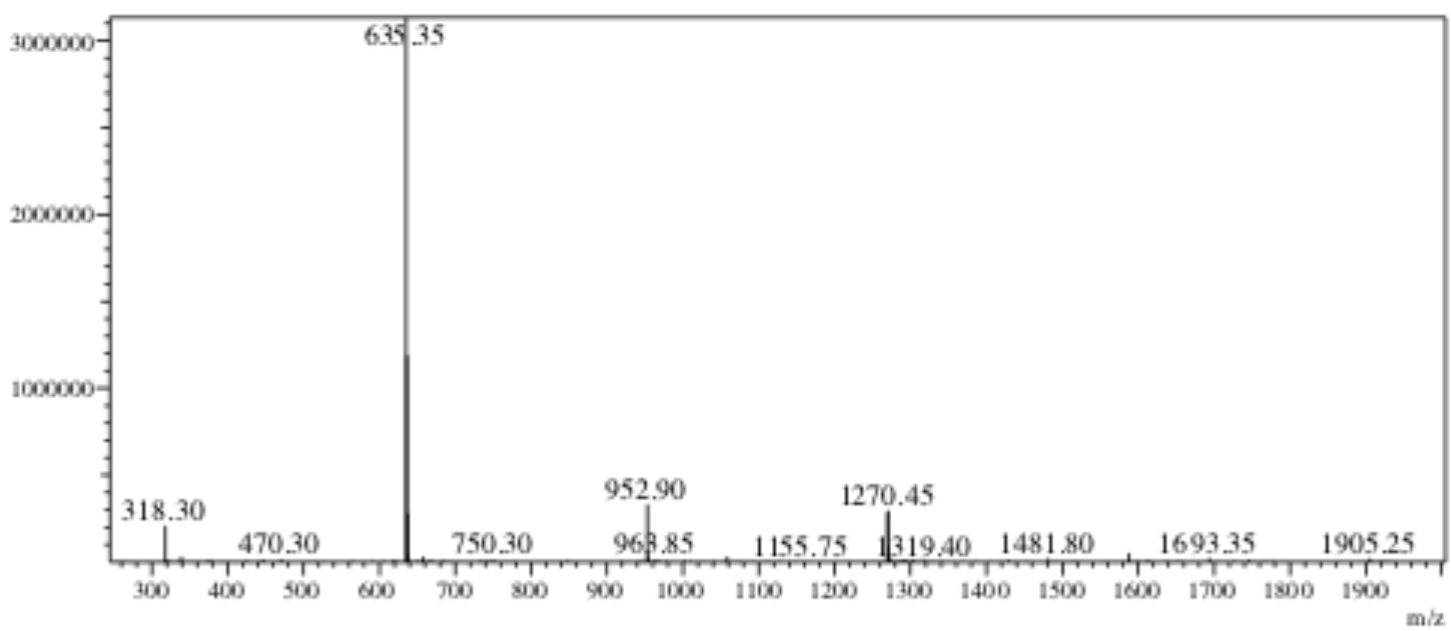




\section{${ }^{1} \mathrm{H}$ NMR of Compound 4}

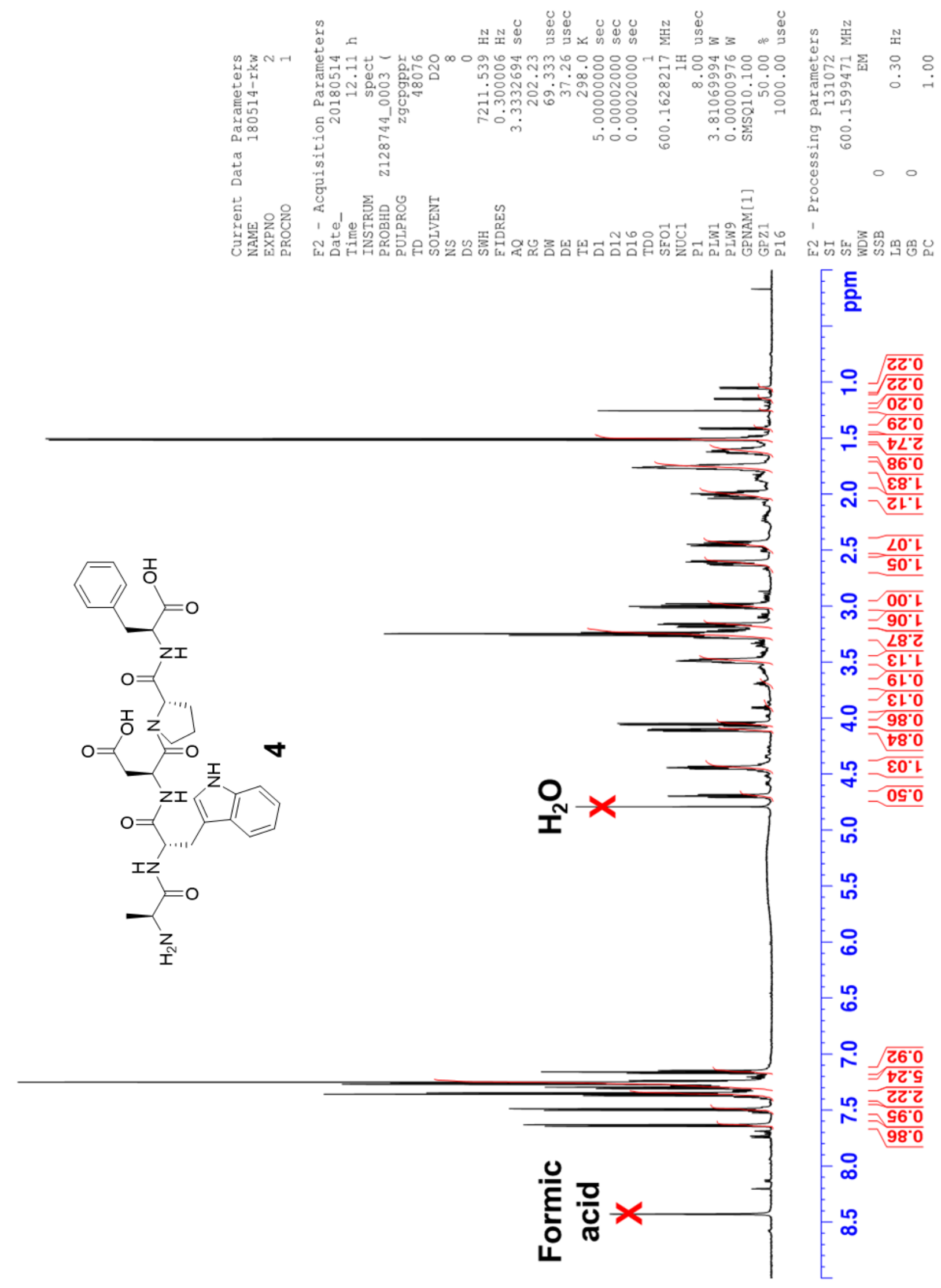




\section{${ }^{1} \mathrm{H}-{ }^{1} \mathrm{H}$ COSY of Compound 4}

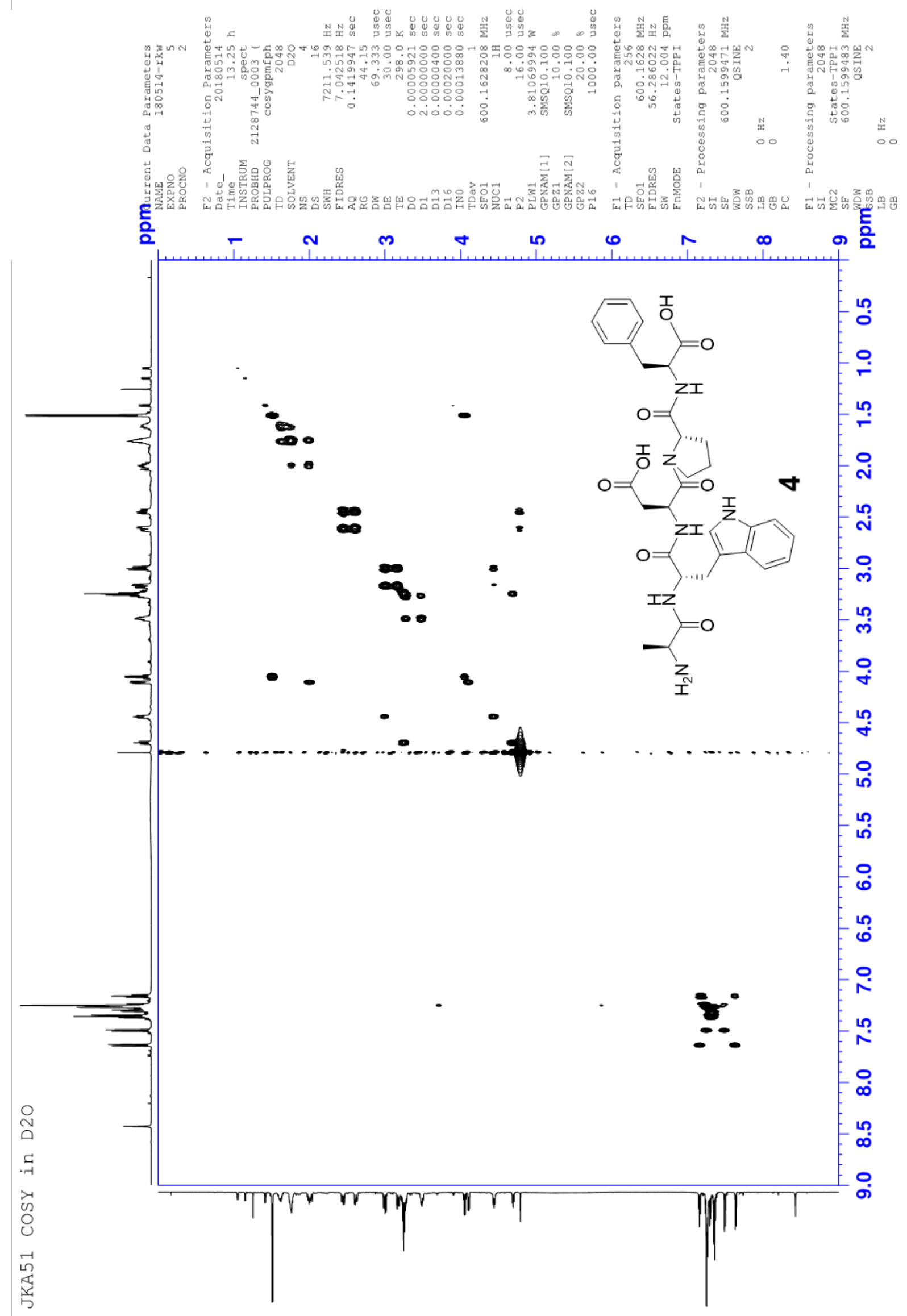




\section{${ }^{1} \mathrm{H}-{ }^{13} \mathrm{C}$ HSQC of Compound 4}

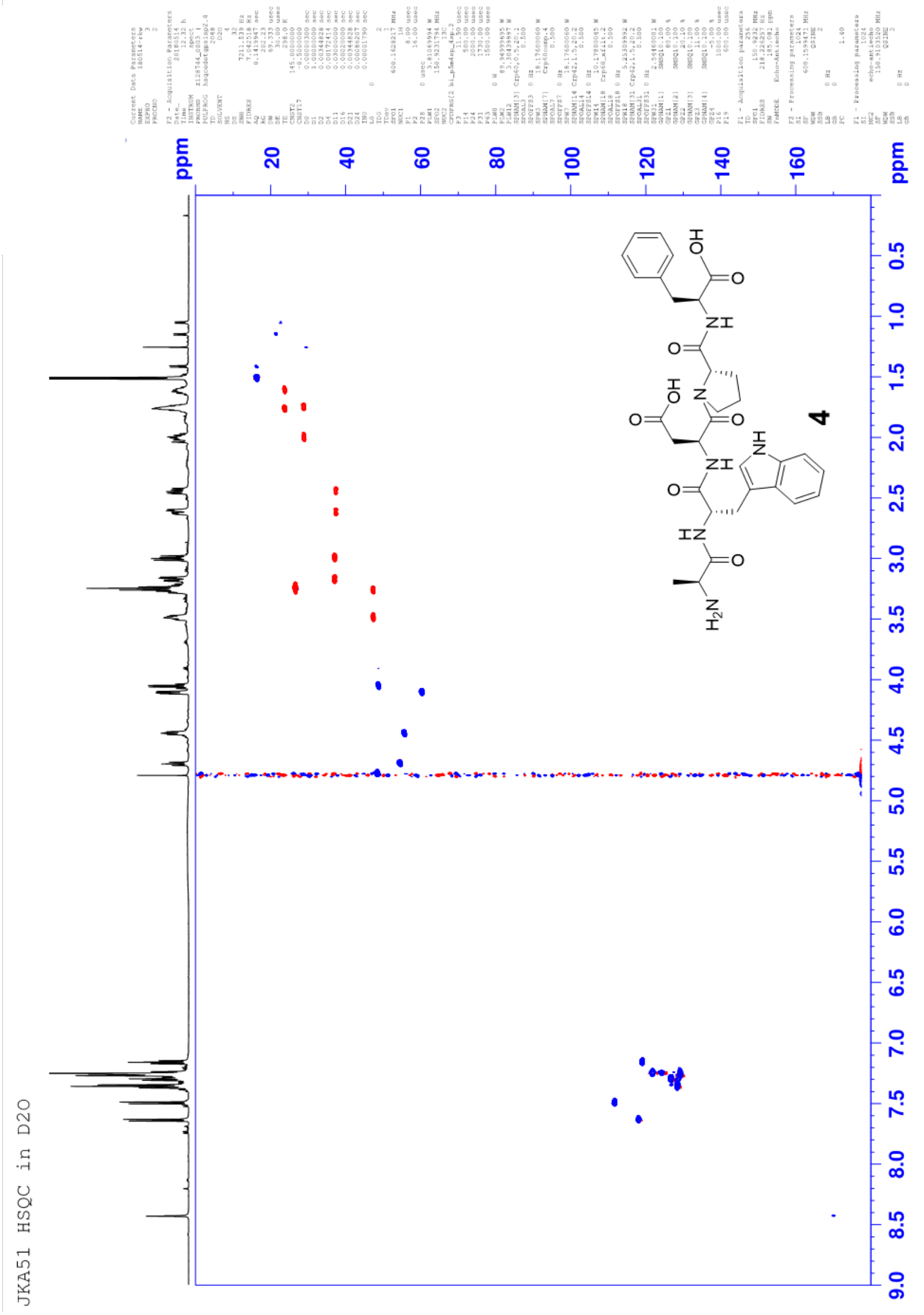




\section{${ }^{1} \mathrm{H}-{ }^{13} \mathrm{C}$ HMBC of Compound 4}

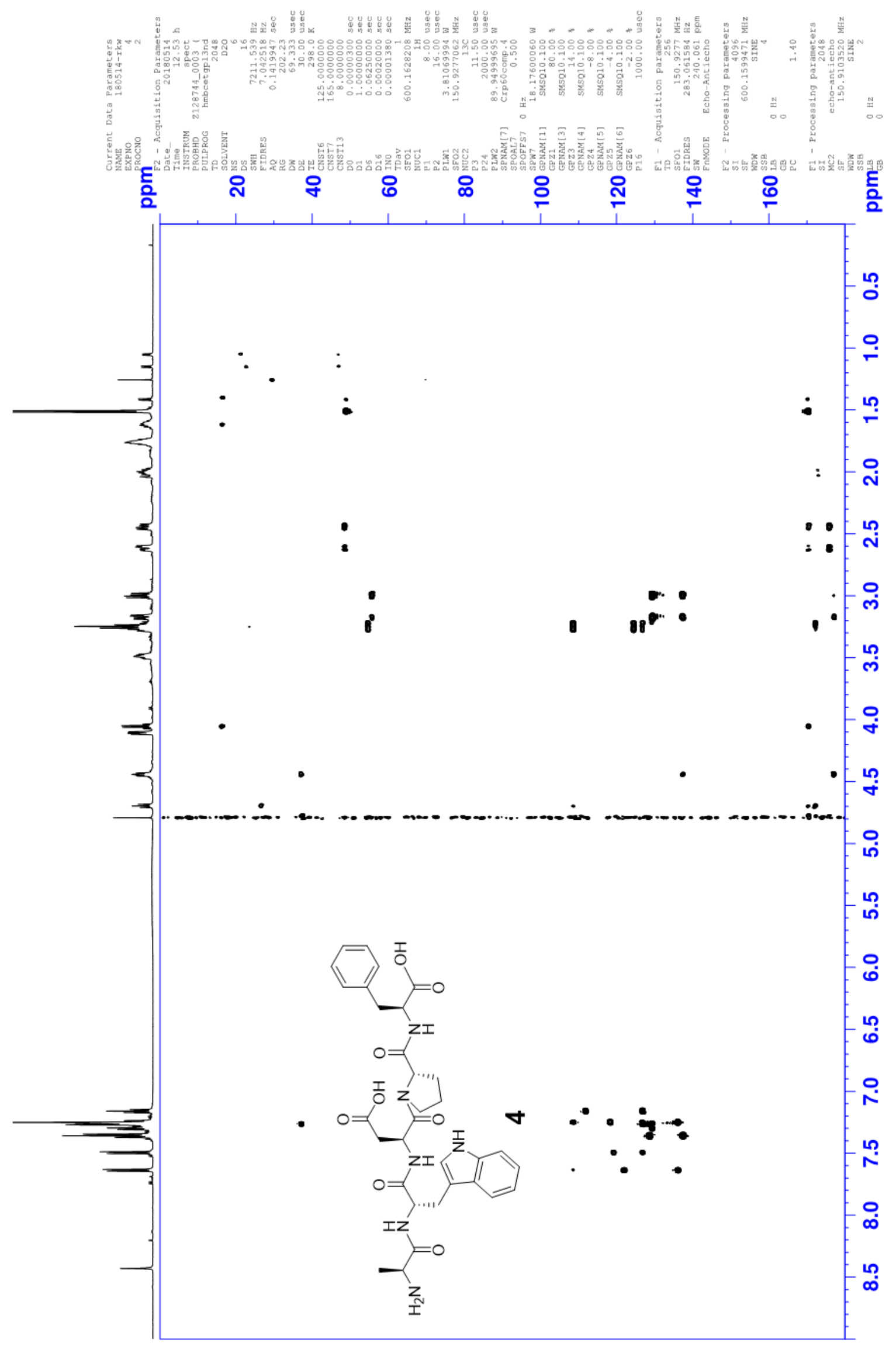


LC/MS of Compound 5<smiles>C[C@H](NC(=O)[C@H](N)CO)C(=O)N[C@@H](CC(=O)O)C(=O)N1CCC(C(=O)N[C@@H](Cc2ccccc2)C(=O)O)C1</smiles>

Chemical Formula: $\mathrm{C}_{24} \mathrm{H}_{33} \mathrm{~N}_{5} \mathrm{O}_{9}$

Exact Mass: 535.23

Molecular Weight: 535.55

mAU

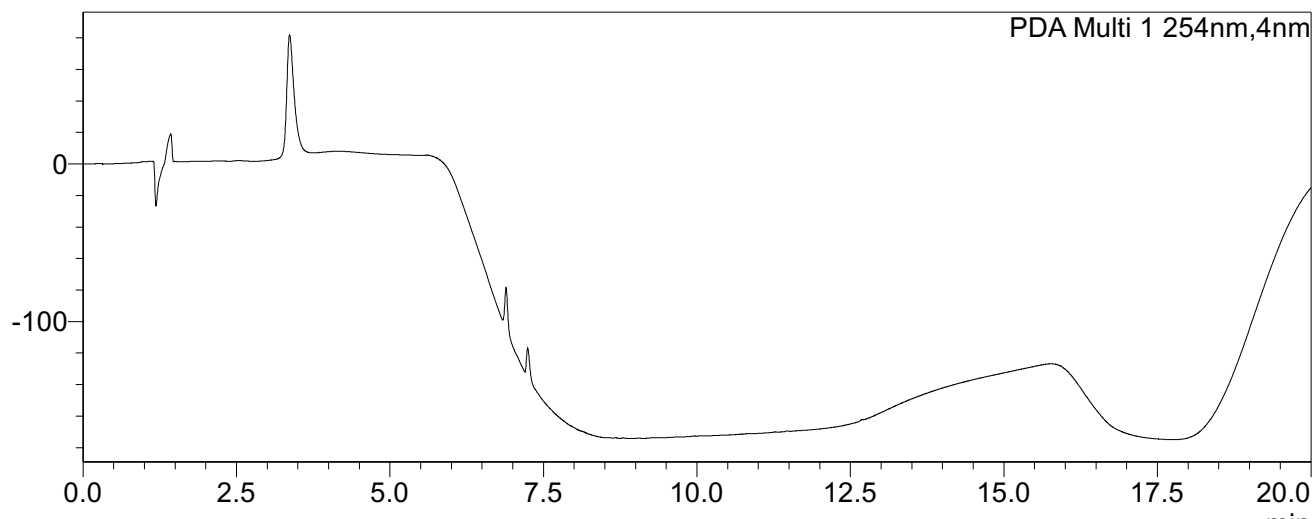

$(x 1,000,000)$

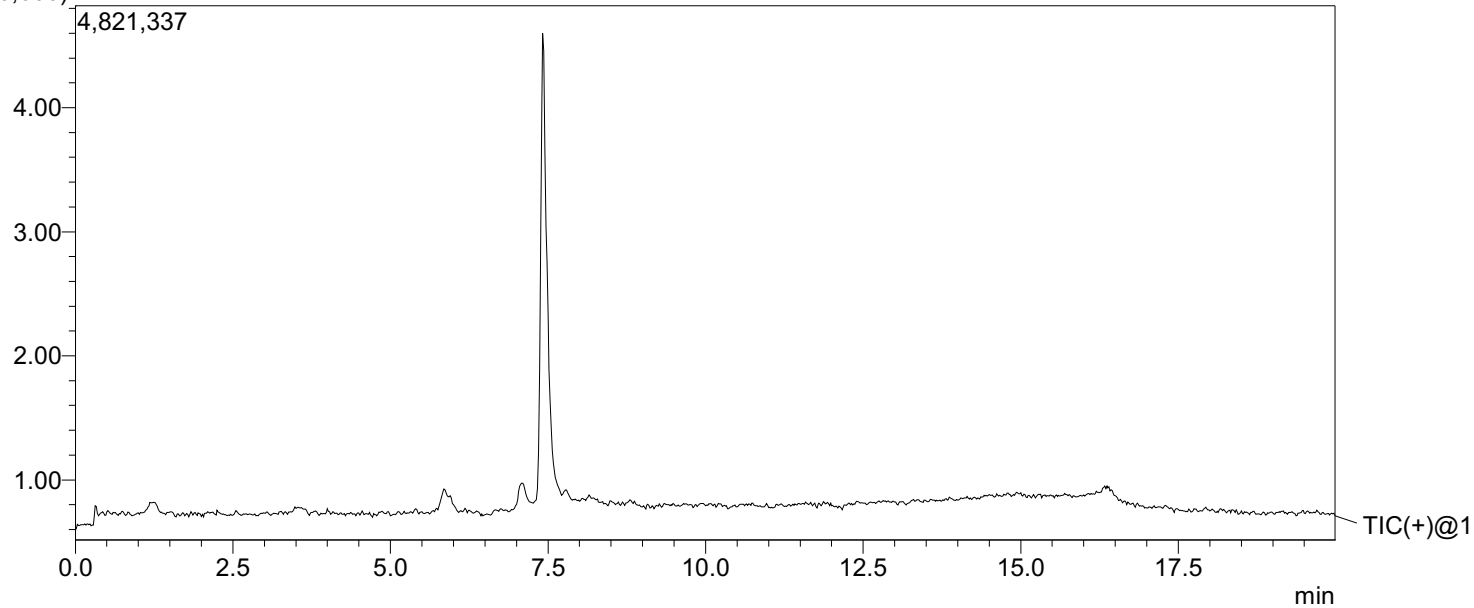

R.Time:7.417(Scan\#:446)

MassPeaks: 1832

Segment 1 - Event 1

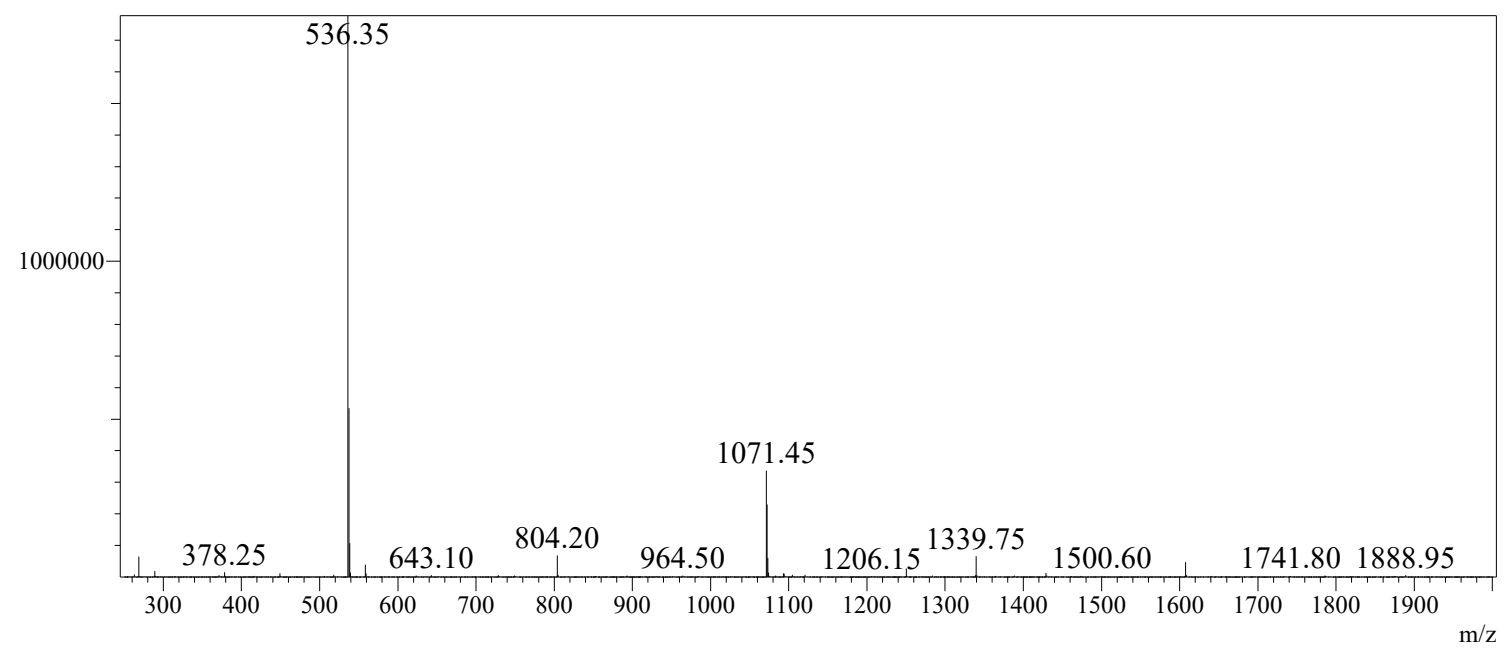




\section{${ }^{1} \mathrm{H}$ NMR of Compound 5}

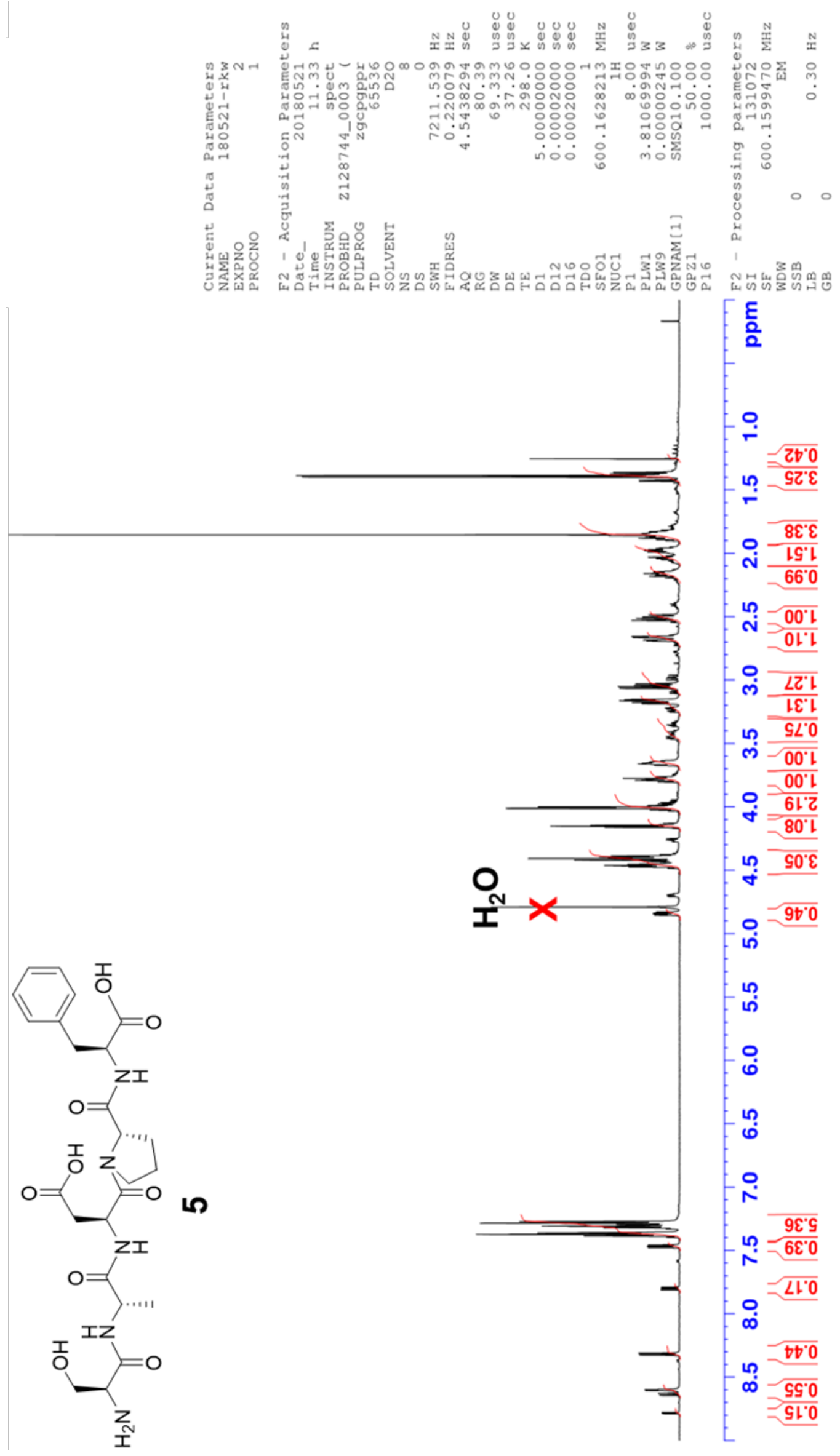




\section{${ }^{1} \mathrm{H}-{ }^{1} \mathrm{H}$ COSY of Compound 5}

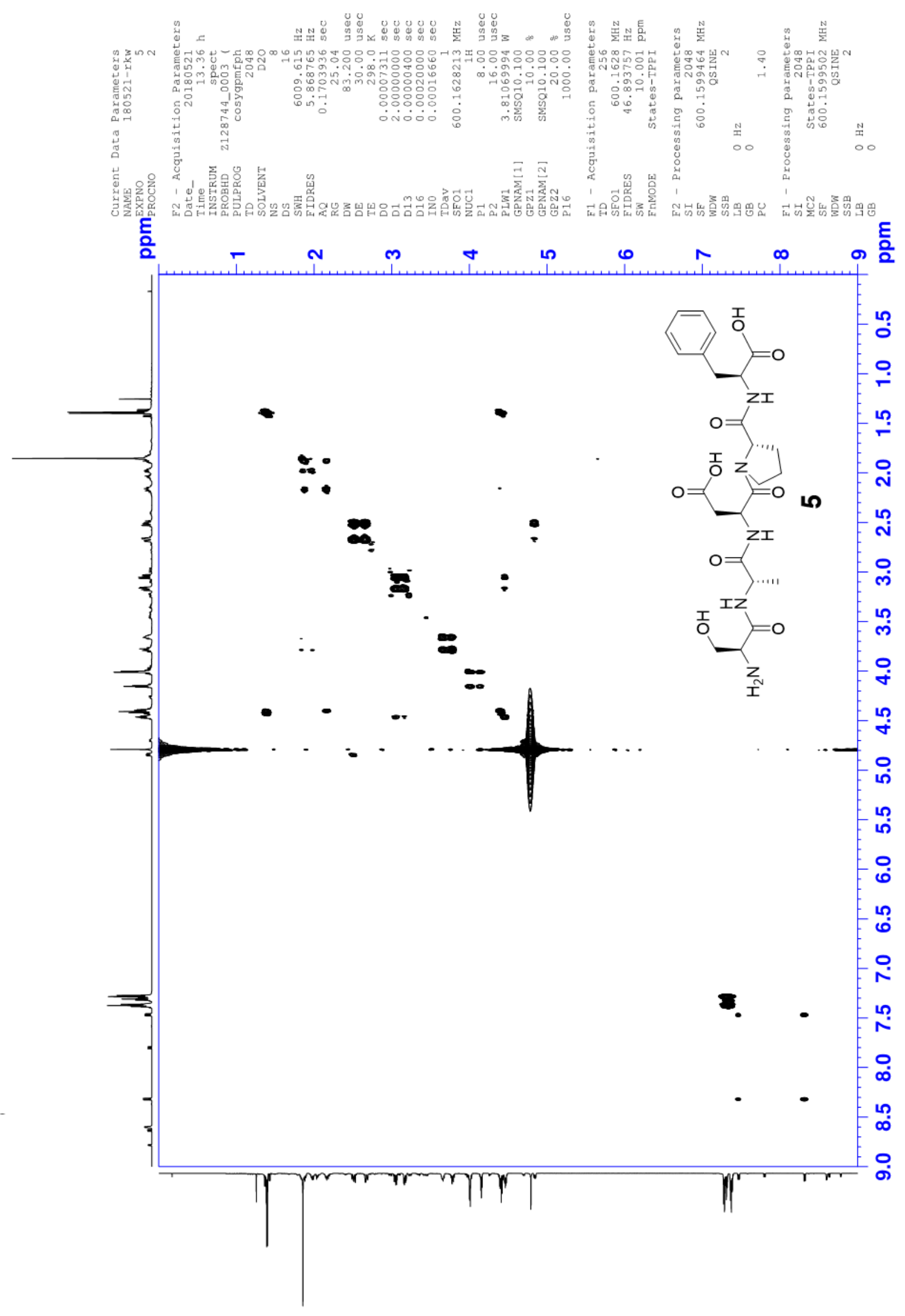




\section{${ }^{1} \mathrm{H}-{ }^{13} \mathrm{C}$ HSQC of Compound 5}
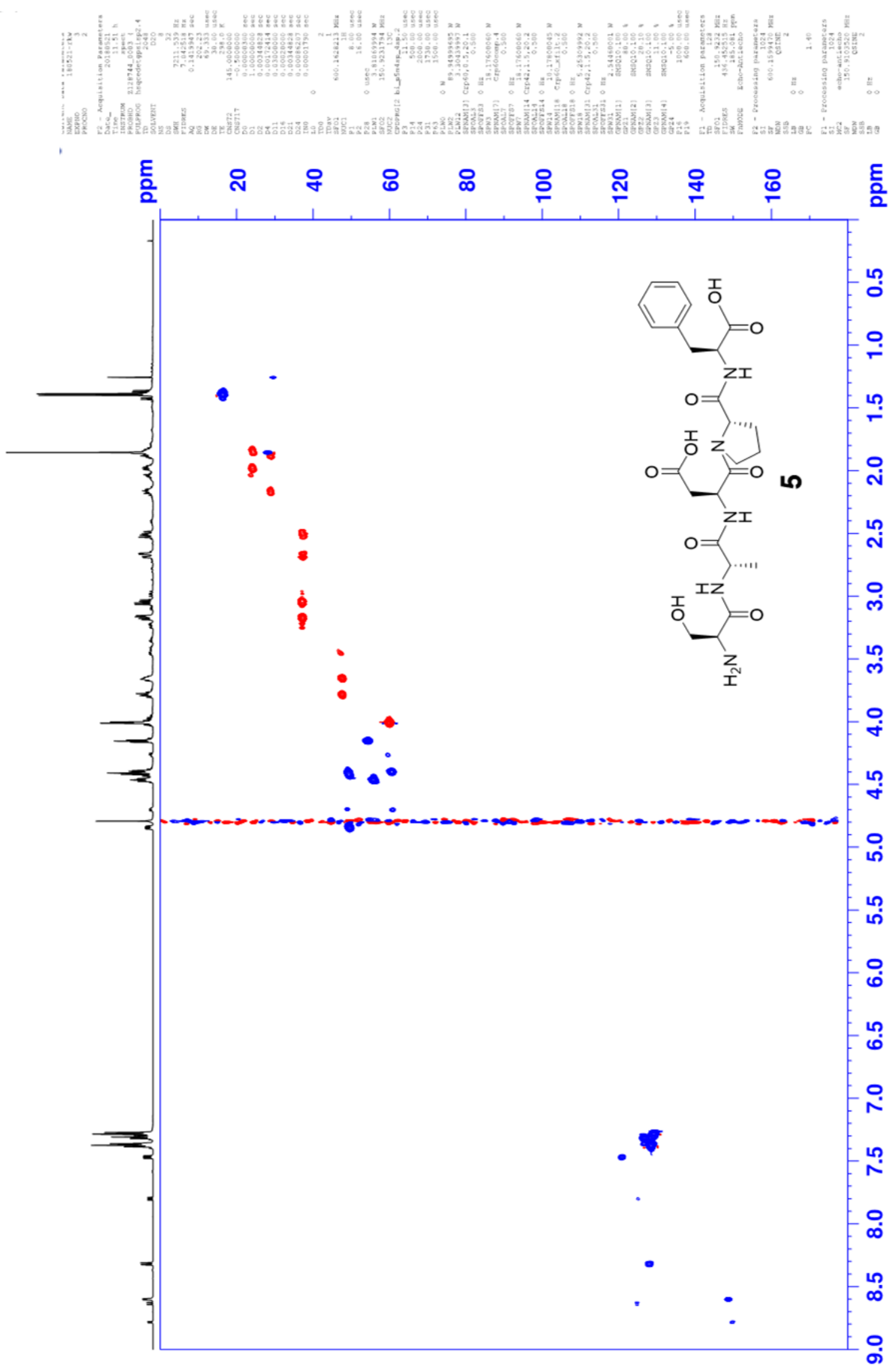


\section{${ }^{1} \mathrm{H}-{ }^{13} \mathrm{C}$ HMBC of Compound 5}

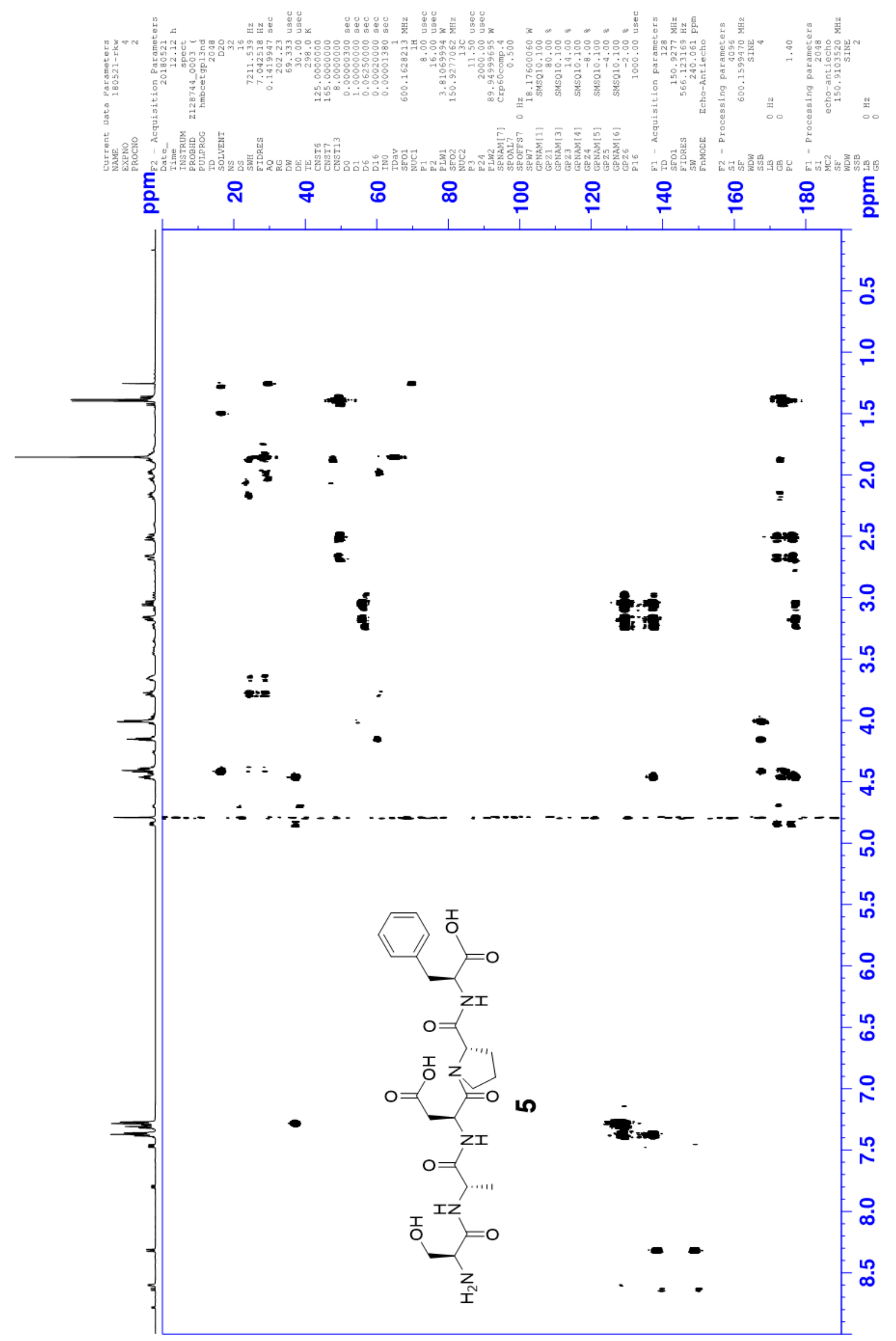




\section{LC/MS of Compound 6}<smiles>C[C@H](NC(=O)C(Cc1c[nH]c2ccccc12)NC(=O)[C@H](N)CO)C(=O)N1CCC[C@H]1C(=O)N[C@@H](Cc1ccccc1)C(=O)O</smiles>

Chemical Formula: $\mathrm{C}_{31} \mathrm{H}_{38} \mathrm{~N}_{6} \mathrm{O}_{7}$

Exact Mass: 606.28

Molecular Weight: 606.68

mAU

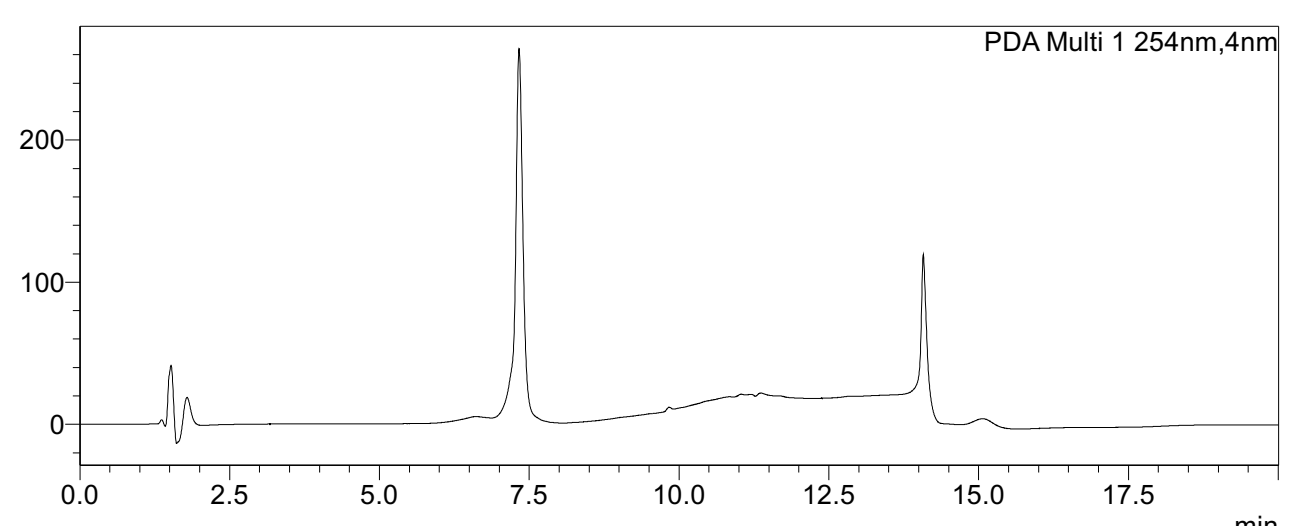

$(\times 1,000,000)$

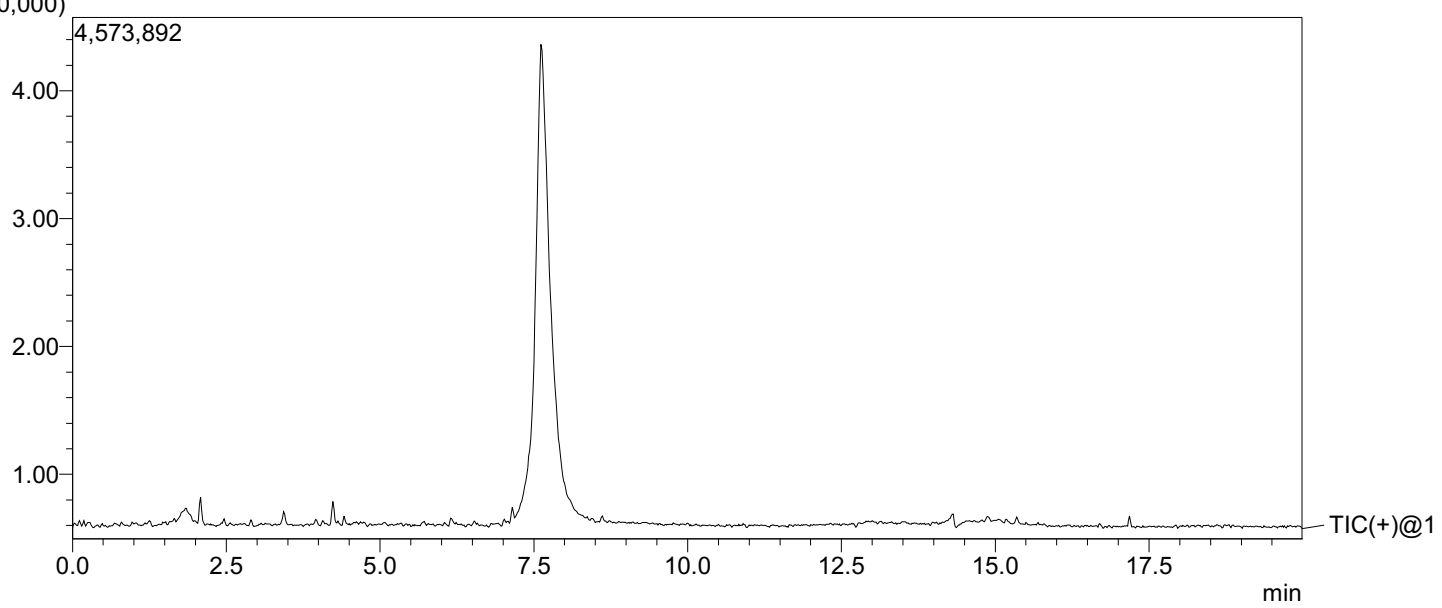

R.Time:7.617(Scan\#:458)

MassPeaks: 1866

Segment 1 - Event 1

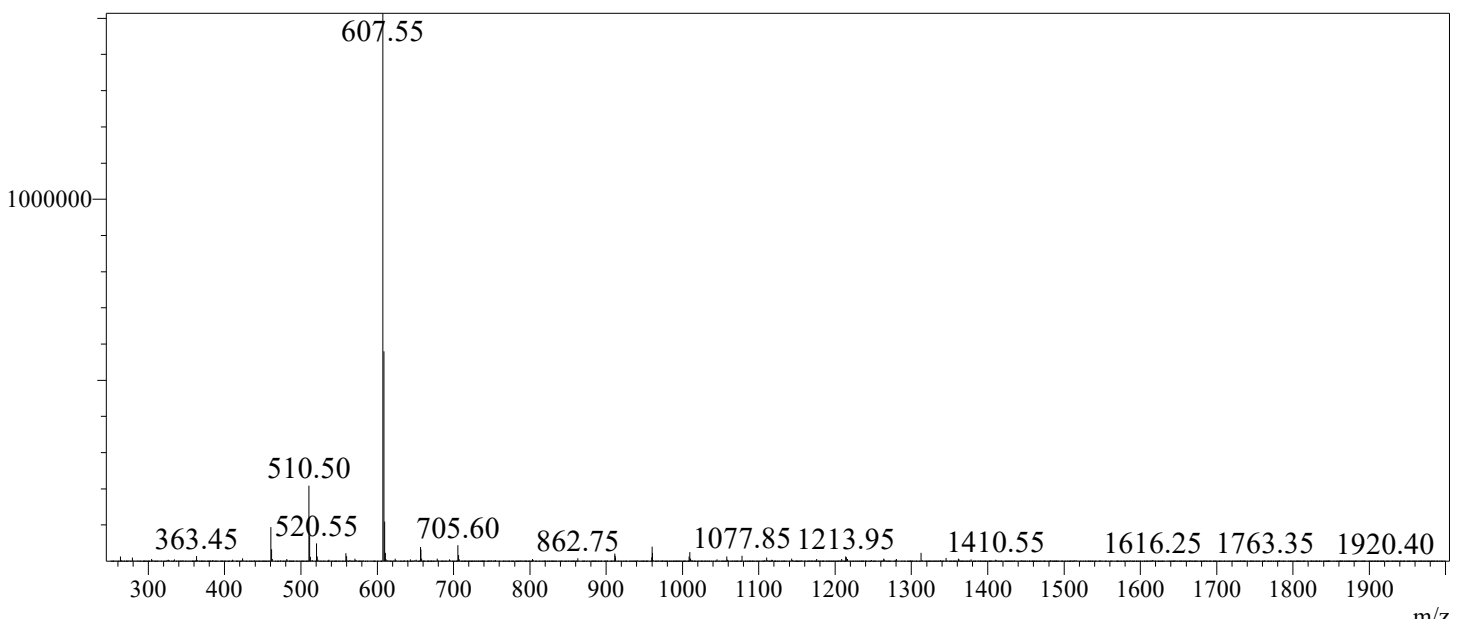




\section{${ }^{1} \mathrm{H}$ NMR of Compound 6}
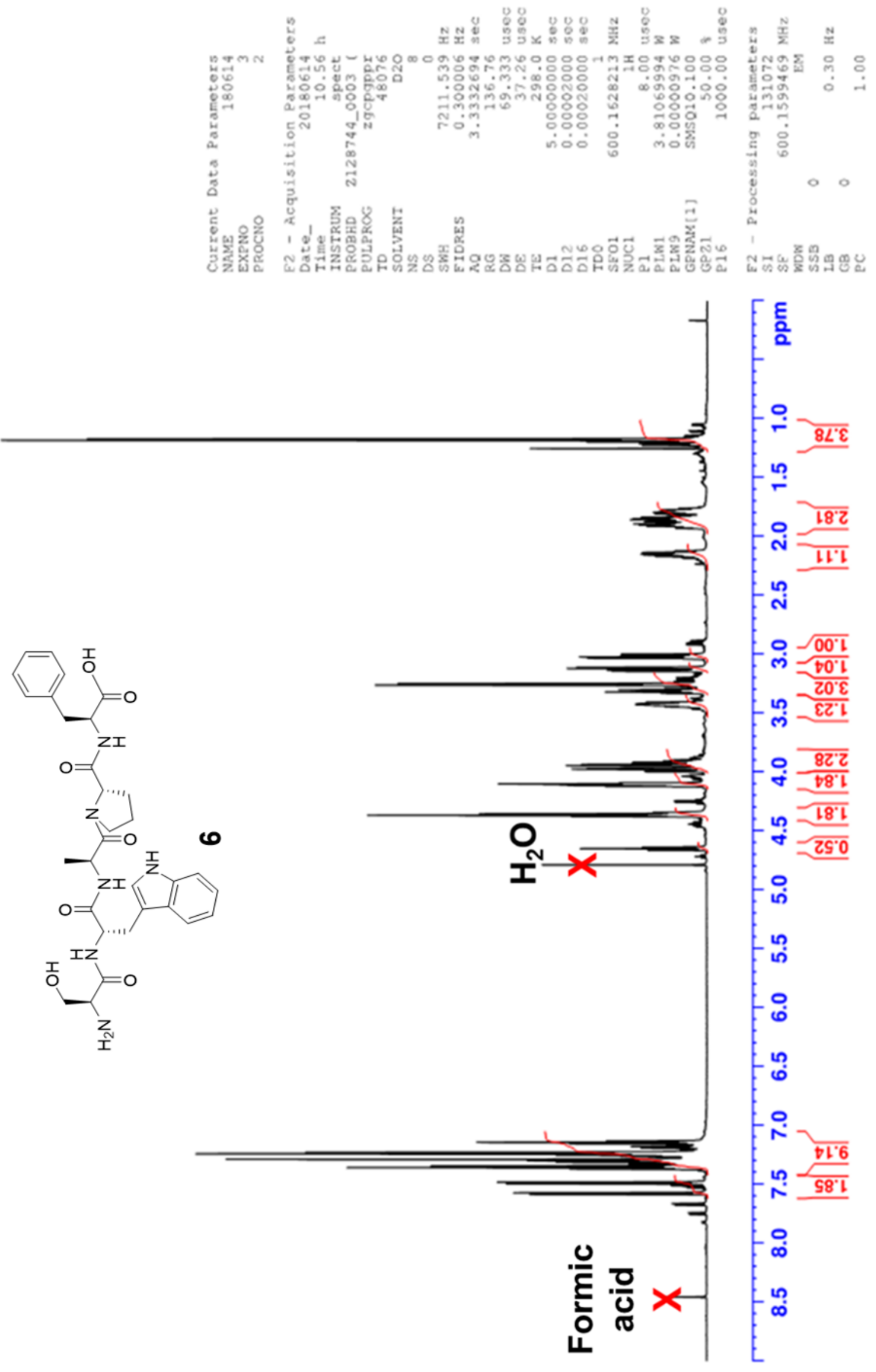


\section{${ }^{1} \mathrm{H}-{ }^{1} \mathrm{H}$ COSY of Compound 6}

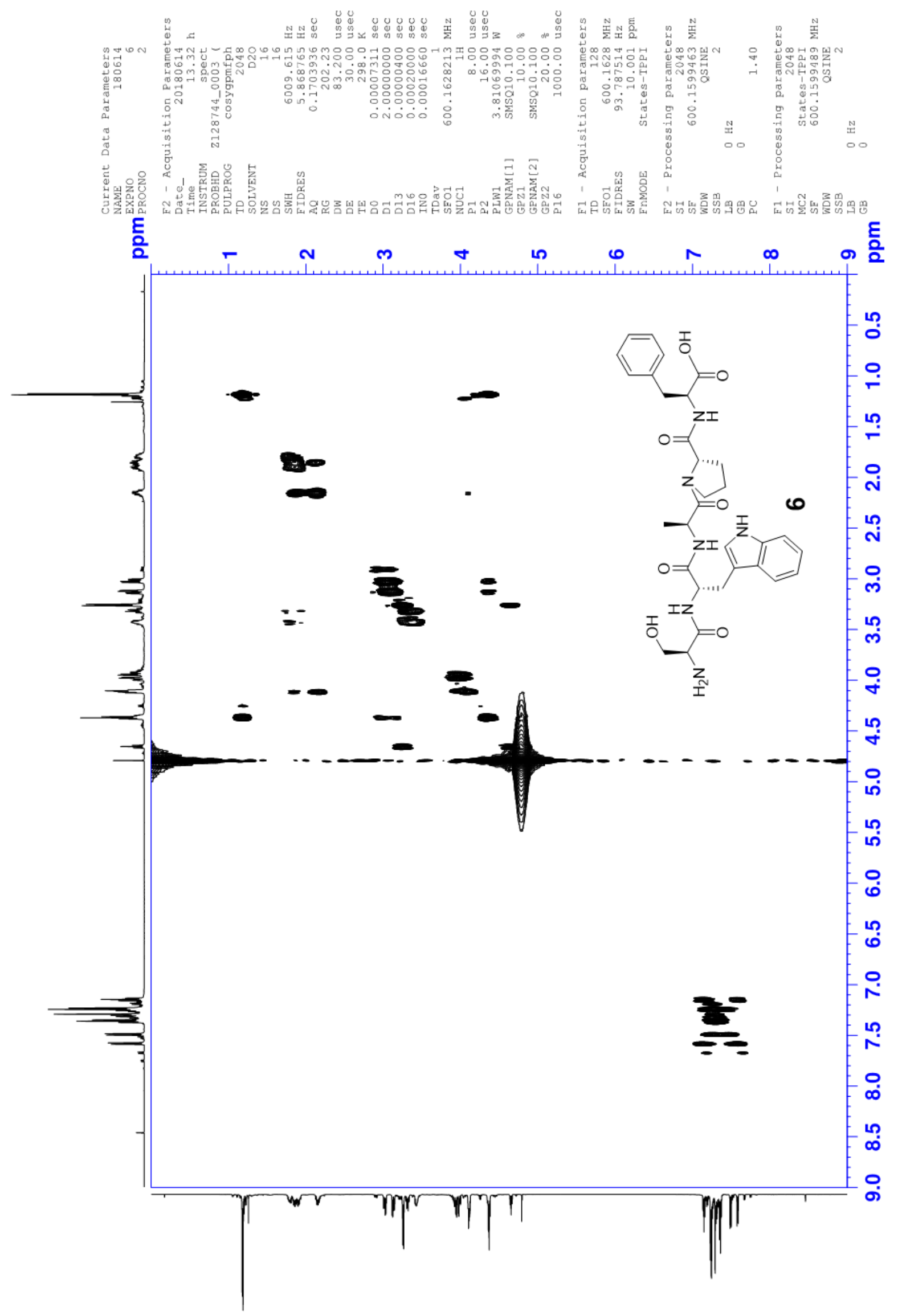




\section{${ }^{1} \mathrm{H}-{ }^{13} \mathrm{C}$ HSQC of Compound 6}

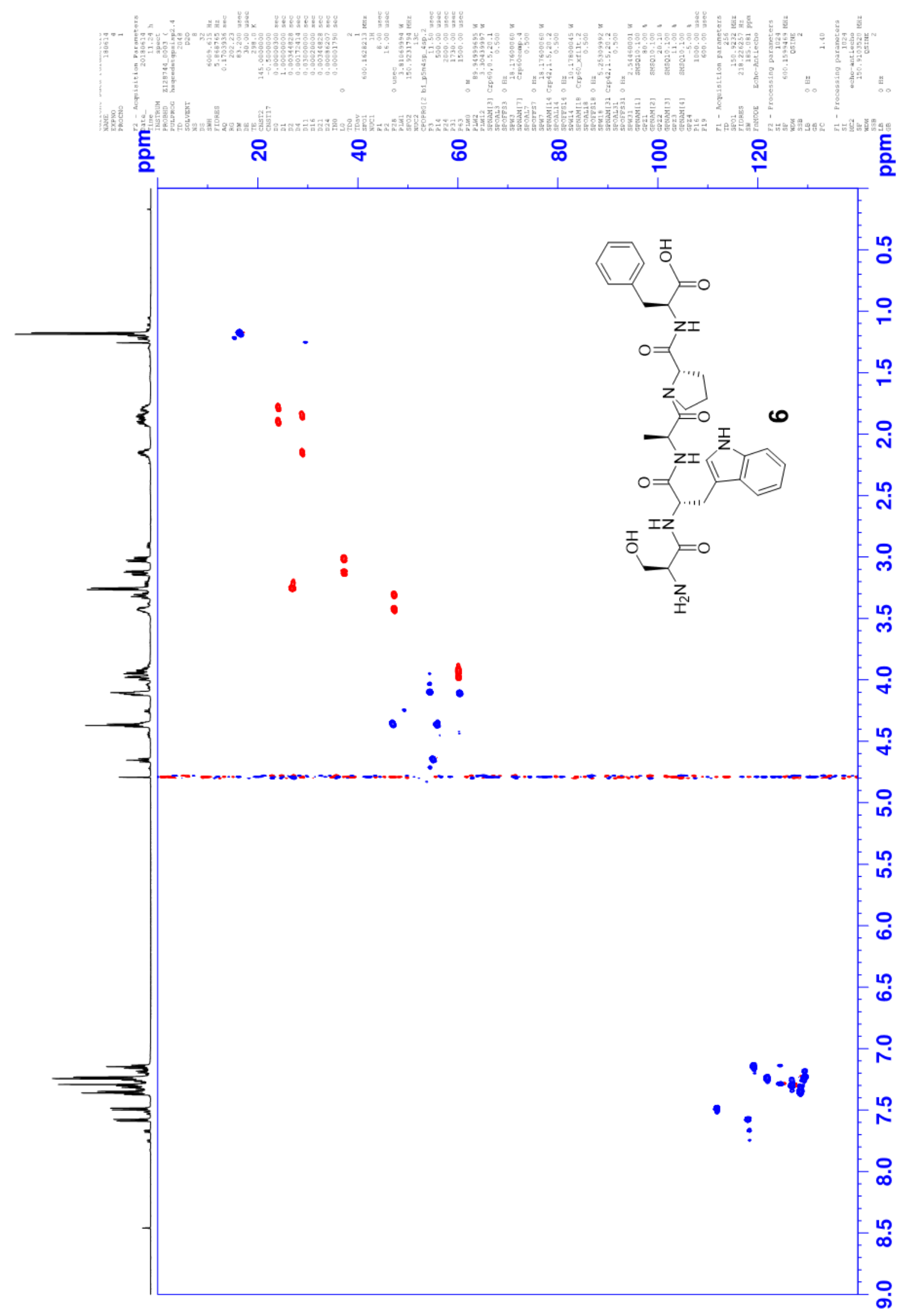




\section{${ }^{1} \mathrm{H}-{ }^{13} \mathrm{C}$ HMBC of Compound 6}

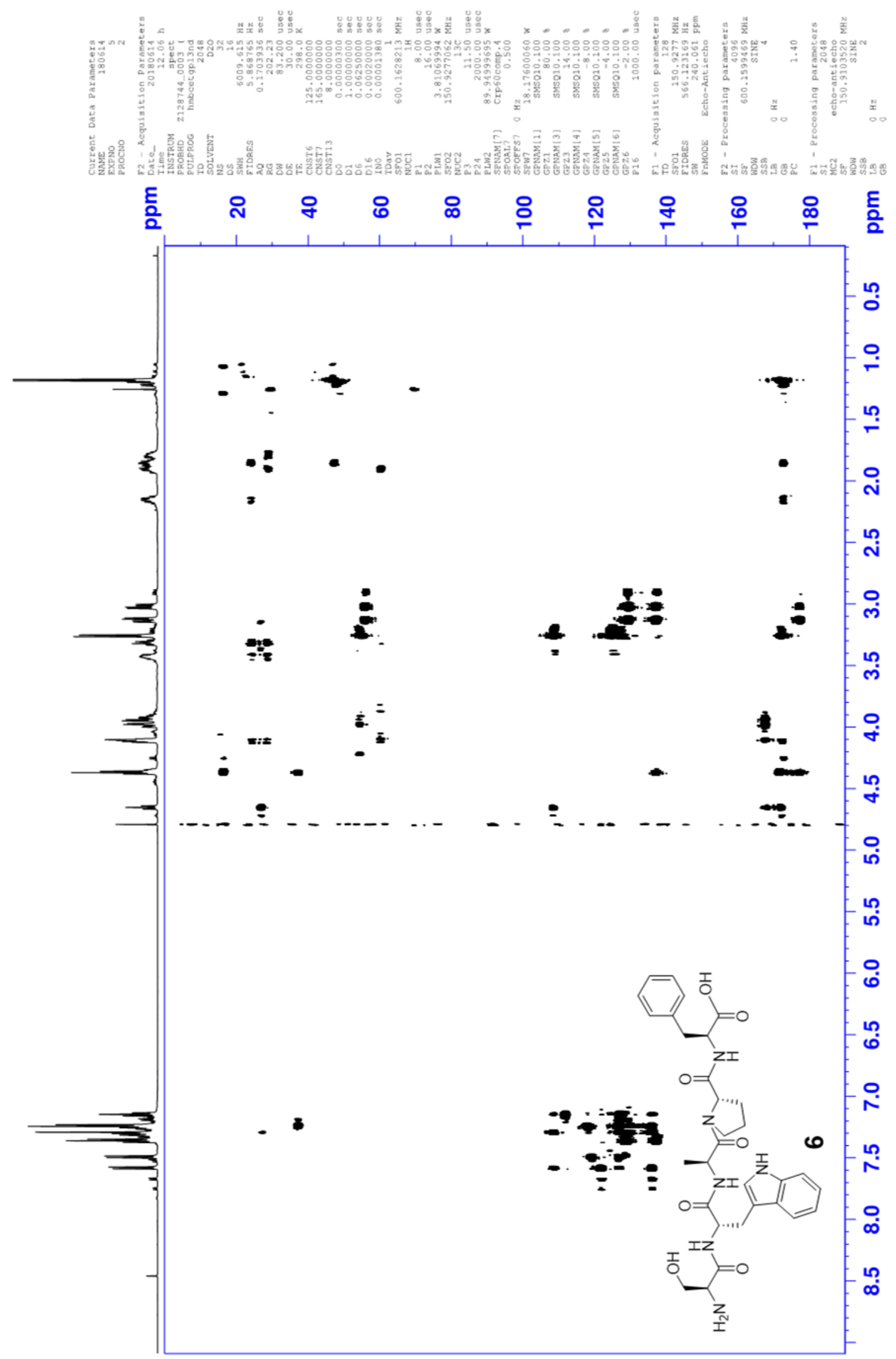




\section{LC/MS of Compound 7}<smiles>C[C@H](NC(=O)[C@H](CC(=O)O)NC(=O)[C@H](Cc1c[nH]c2ccccc12)NC(=O)[C@H](N)CO)C(=O)N[C@@H](Cc1ccccc1)C(=O)O</smiles>

Chemical Formula: $\mathrm{C}_{30} \mathrm{H}_{36} \mathrm{~N}_{6} \mathrm{O}_{9}$

Exact Mass: 624.25

Molecular Weight: 624.65

mAU

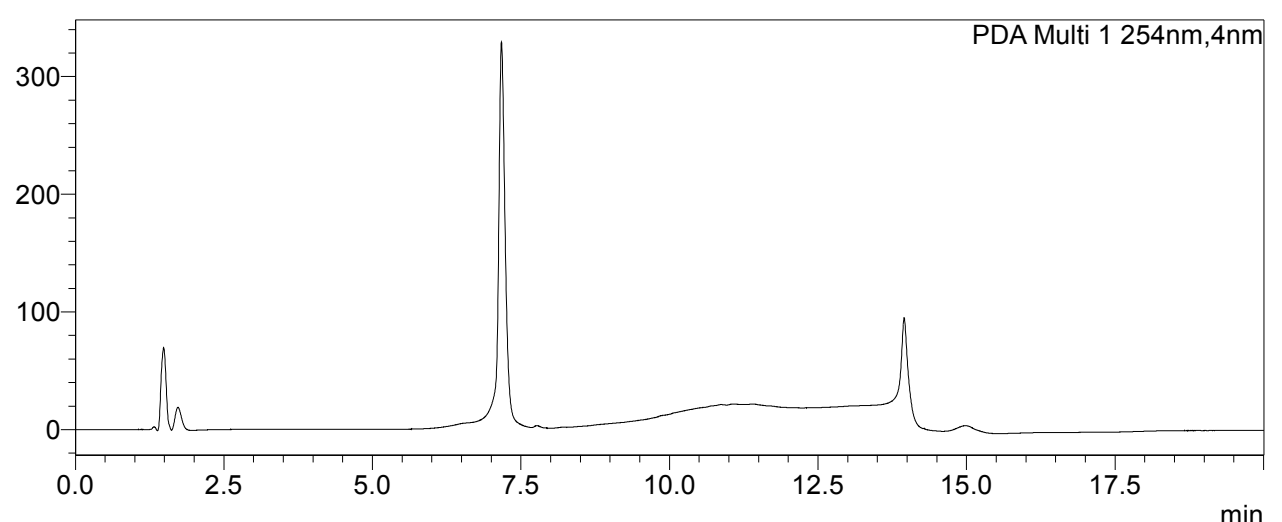

$(\times 1,000,000)$

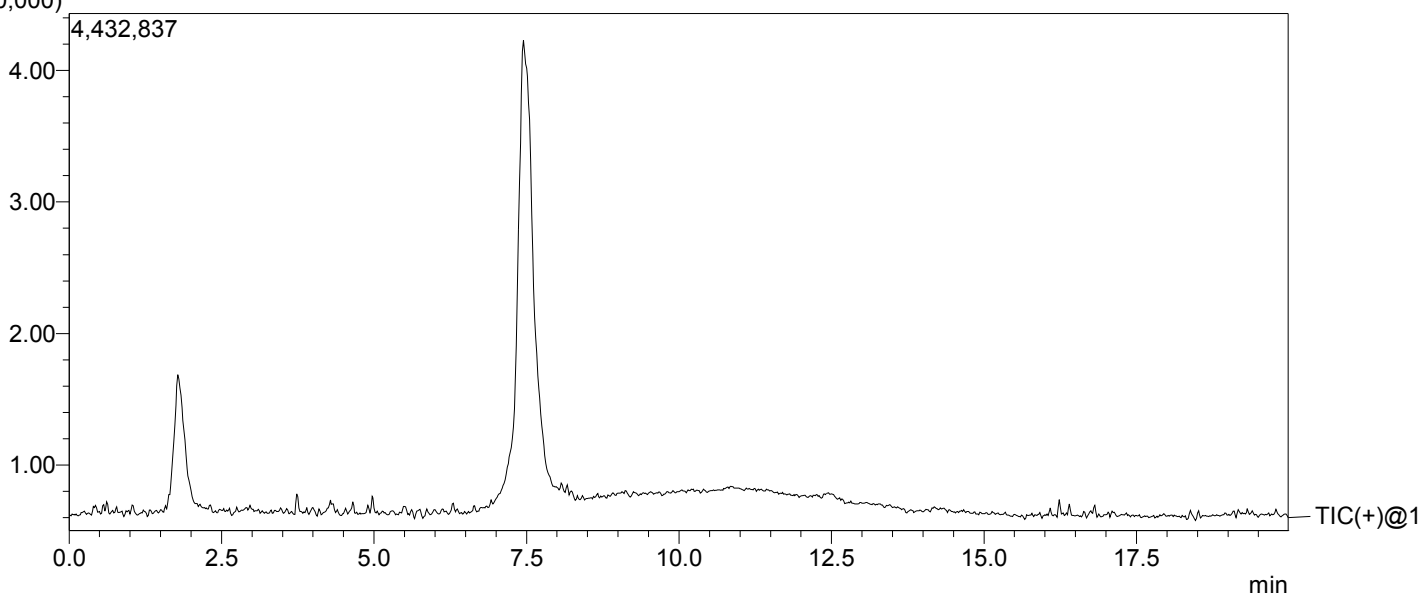

R.Time:1.783(Scan\#:108)

MassPeaks:1794

Segment 1 - Event 1

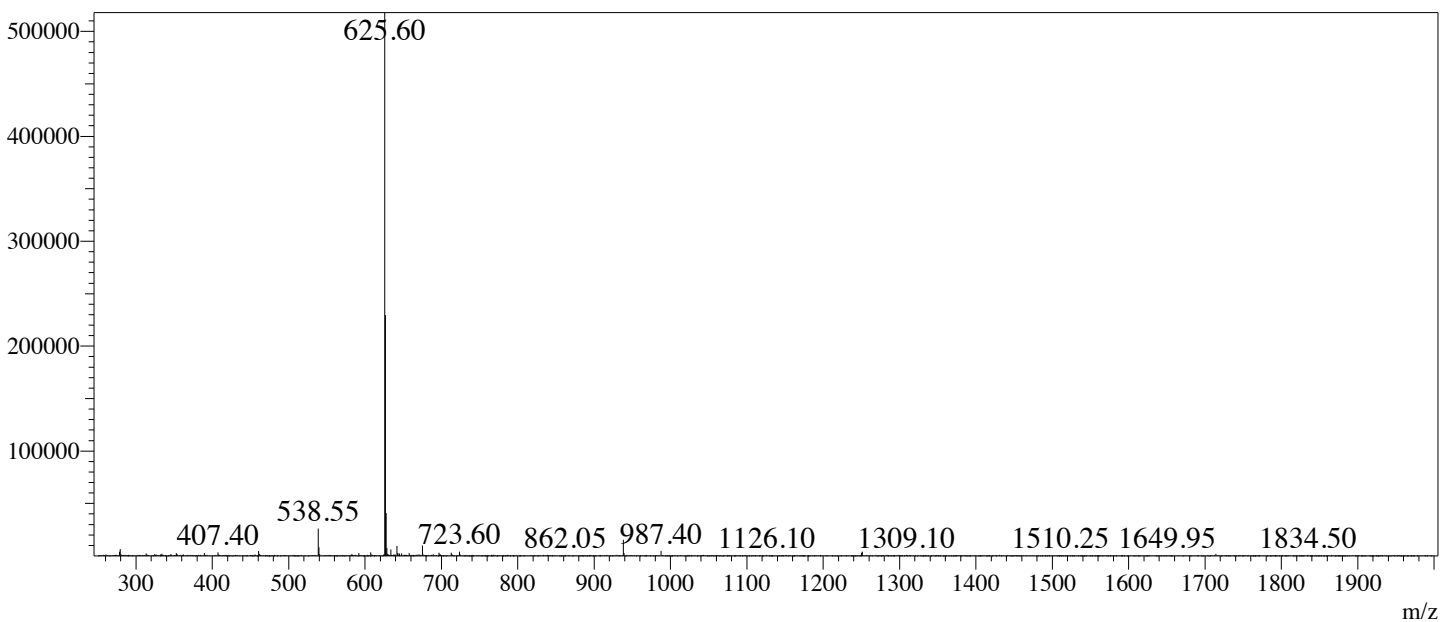


R.Time:7.450(Scant:448)
Masspeaks:1808

Serment I. Event I

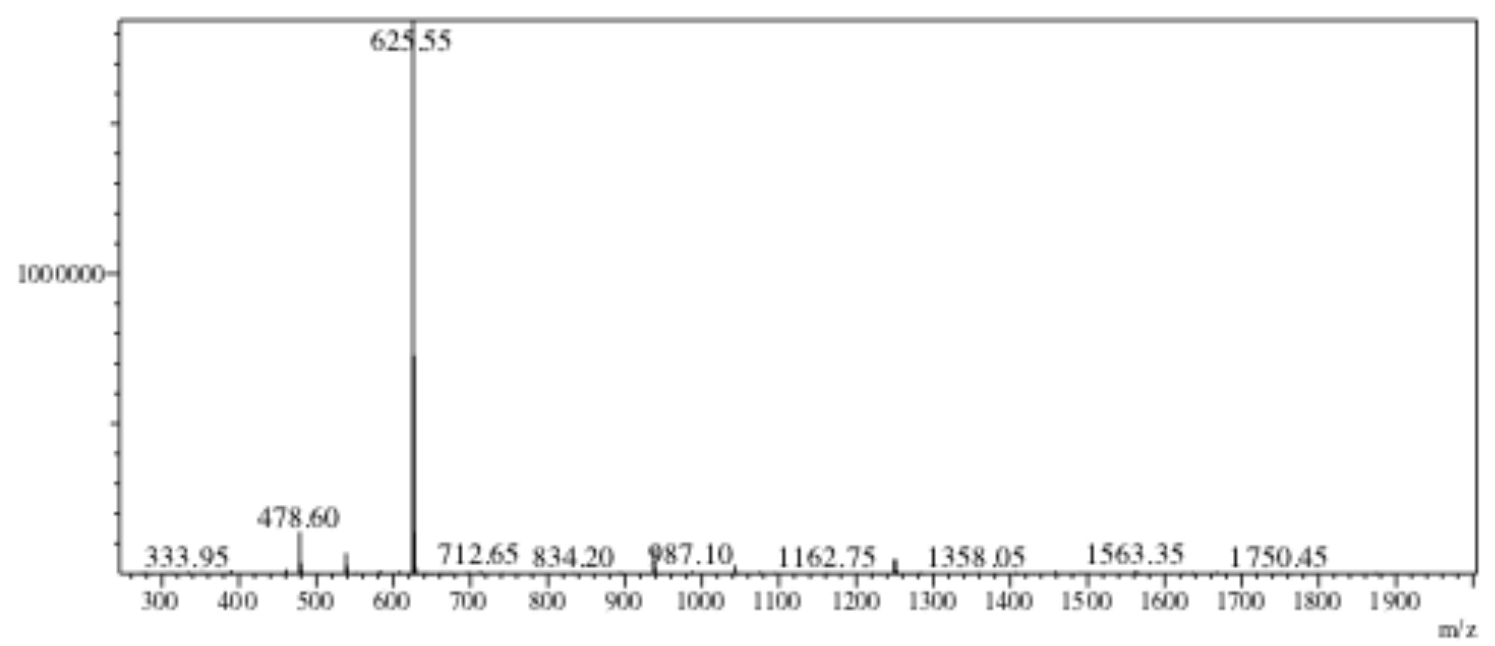




\section{${ }^{1} \mathrm{H}$ NMR of Compound 7}
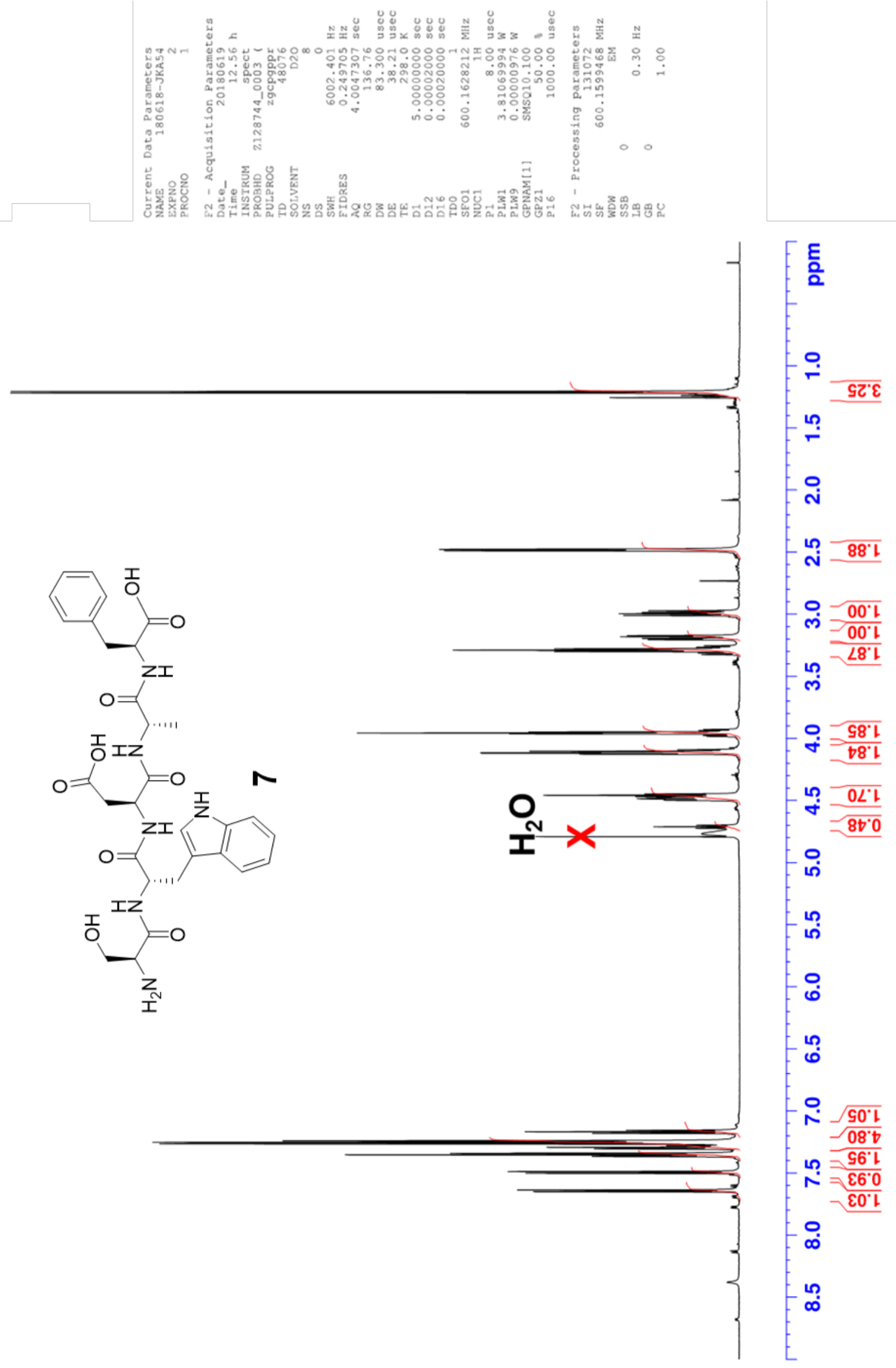


\section{${ }^{1} \mathrm{H}-{ }^{1} \mathrm{H}$ COSY of Compound 7}

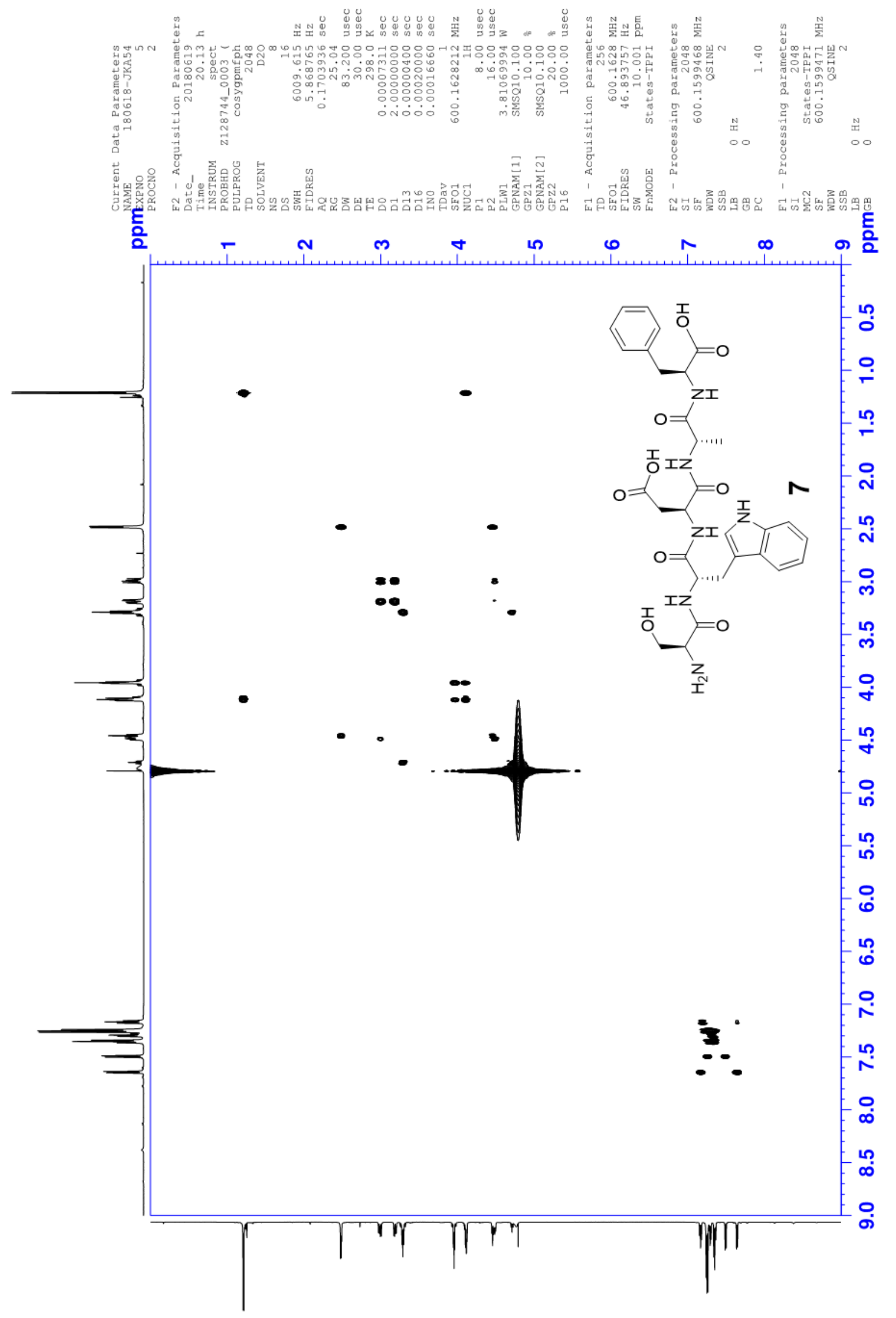




\section{${ }^{1} \mathrm{H}-{ }^{13} \mathrm{C}$ HSQC of Compound 7}

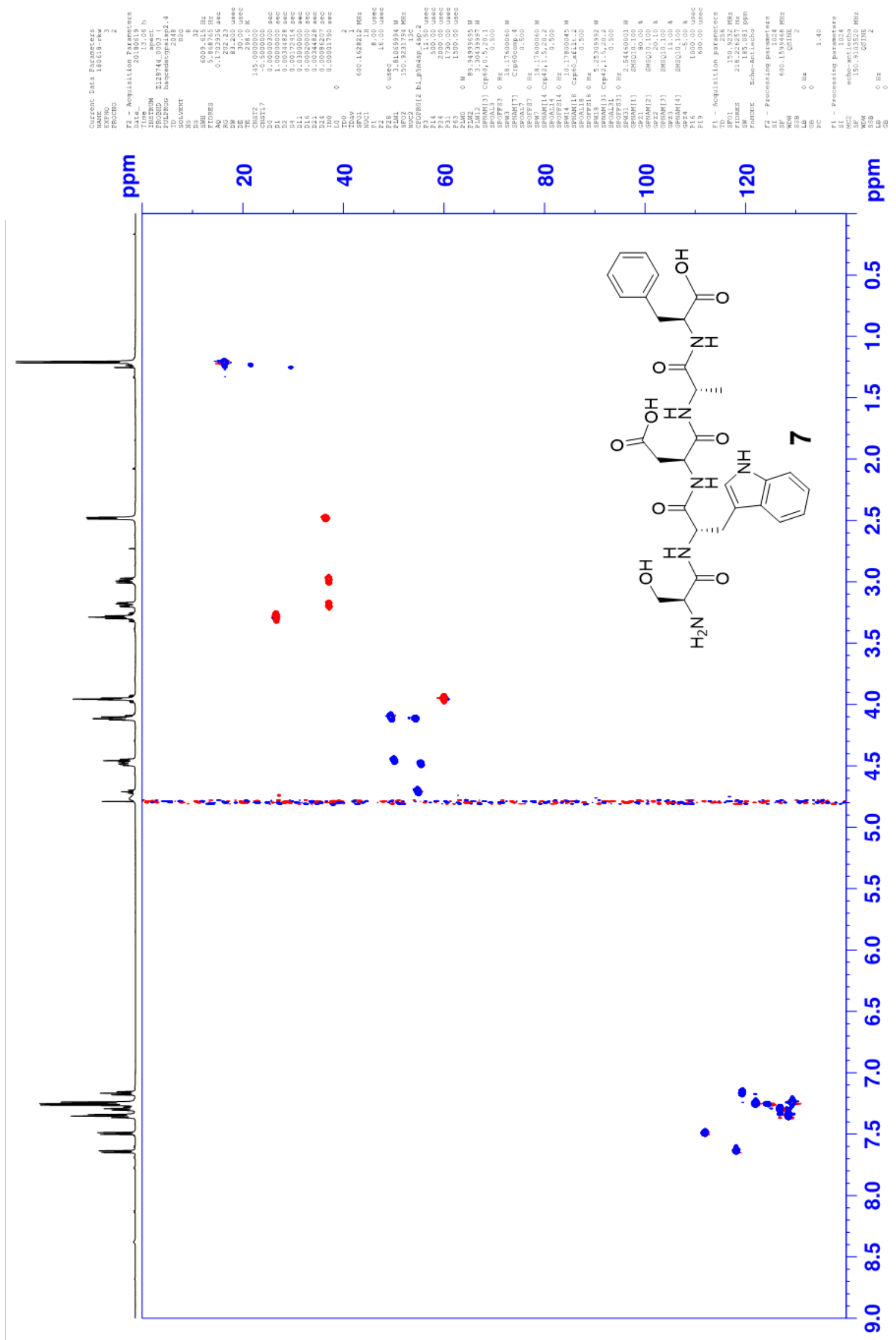




\section{${ }^{1} \mathrm{H}-{ }^{13} \mathrm{C}$ HMBC of Compound 7}

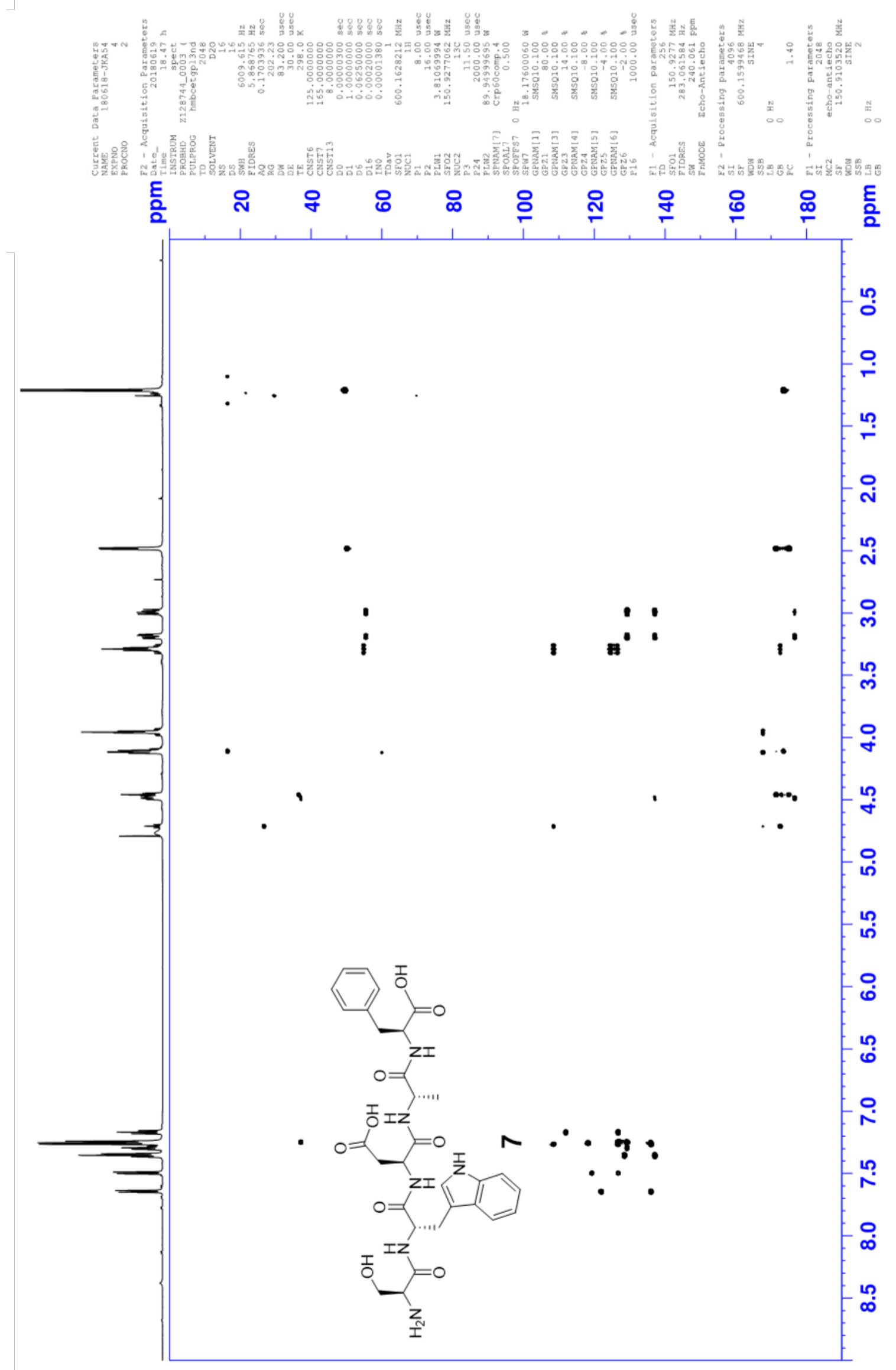




\section{LC/MS of Compound 8}<smiles></smiles>

Chemical Formula: $\mathrm{C}_{26} \mathrm{H}_{34} \mathrm{~N}_{6} \mathrm{O}_{9}$

Exact Mass: 574.24

Molecular Weight: 574.59

mAU

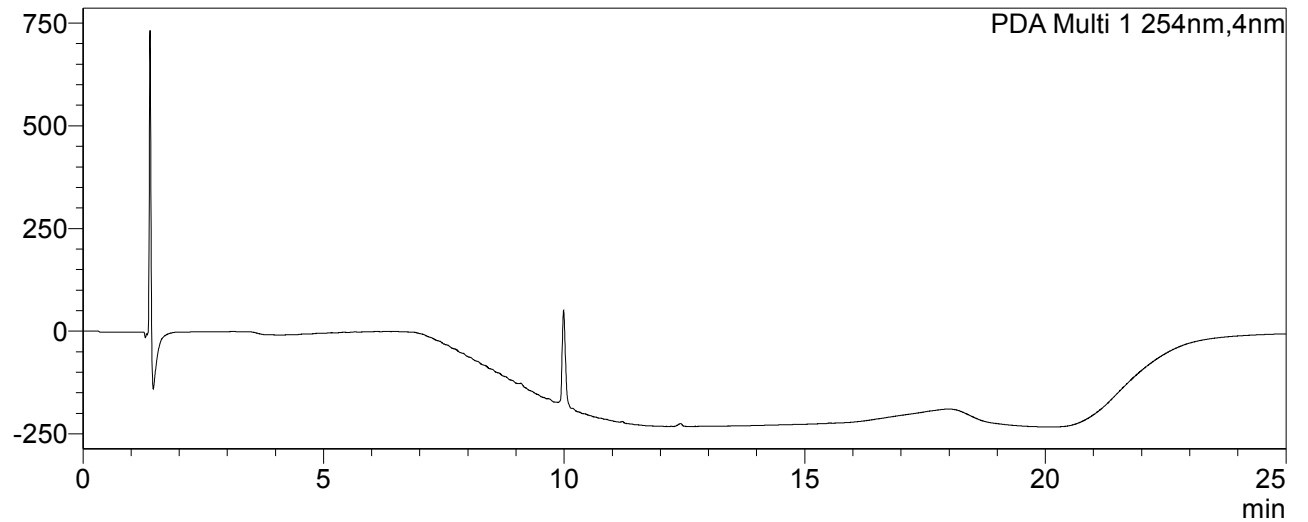

$(x 10,000,000)$

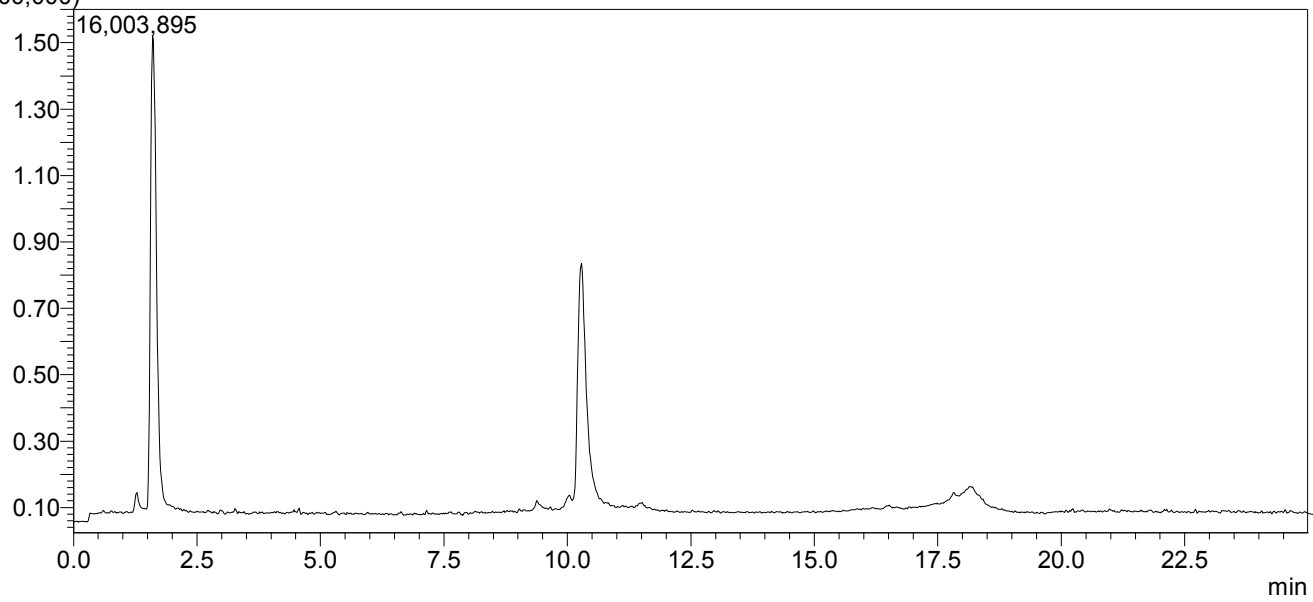

R.Time:1.600(Scan\#:97)

MassPeaks: 1916

Segment 1 - Event 1

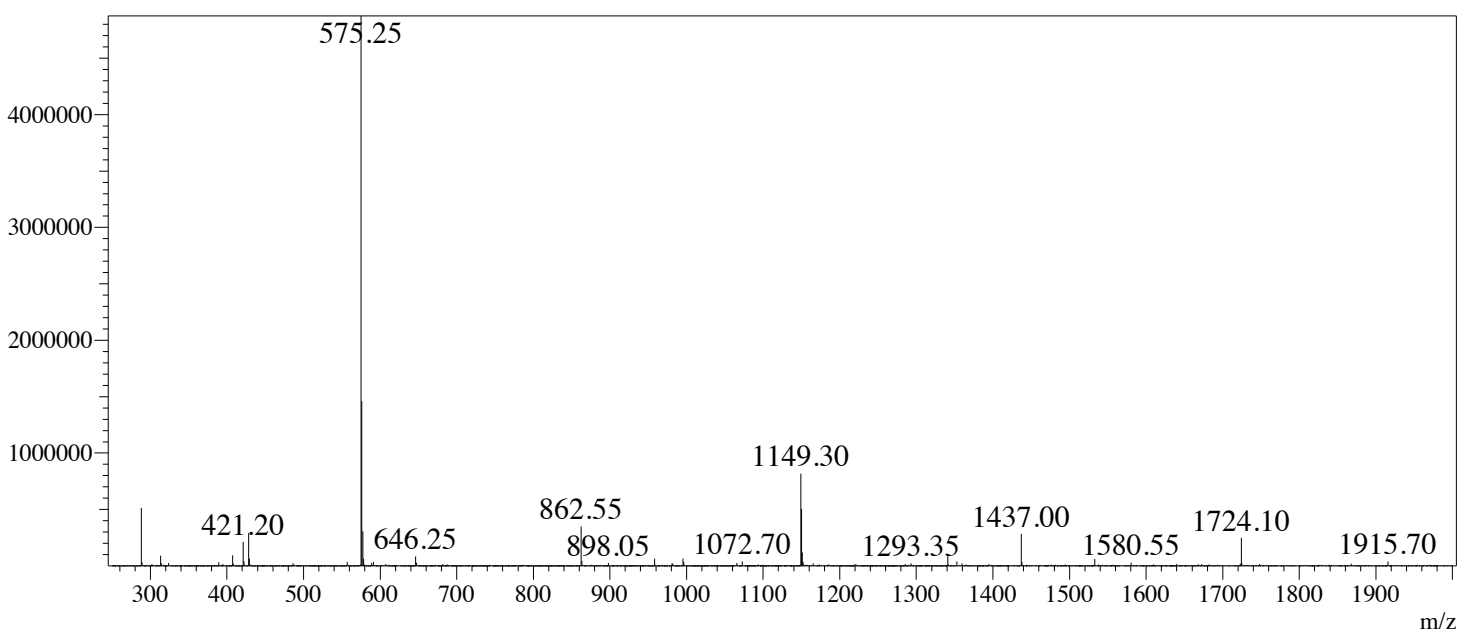


R.Time:10.283(Scan\#:618)

MassPeaks:1893

Segment 1 - Event 1

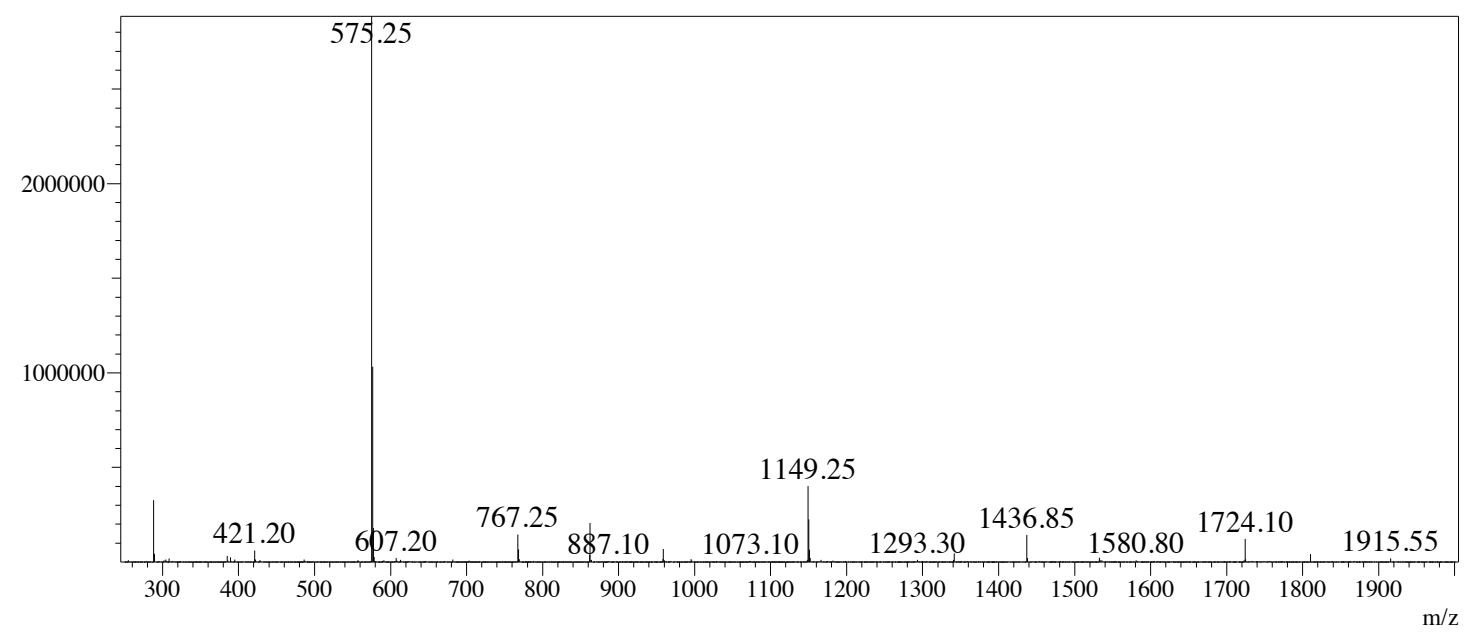




\section{${ }^{1} \mathrm{H}$ NMR of Compound 8}

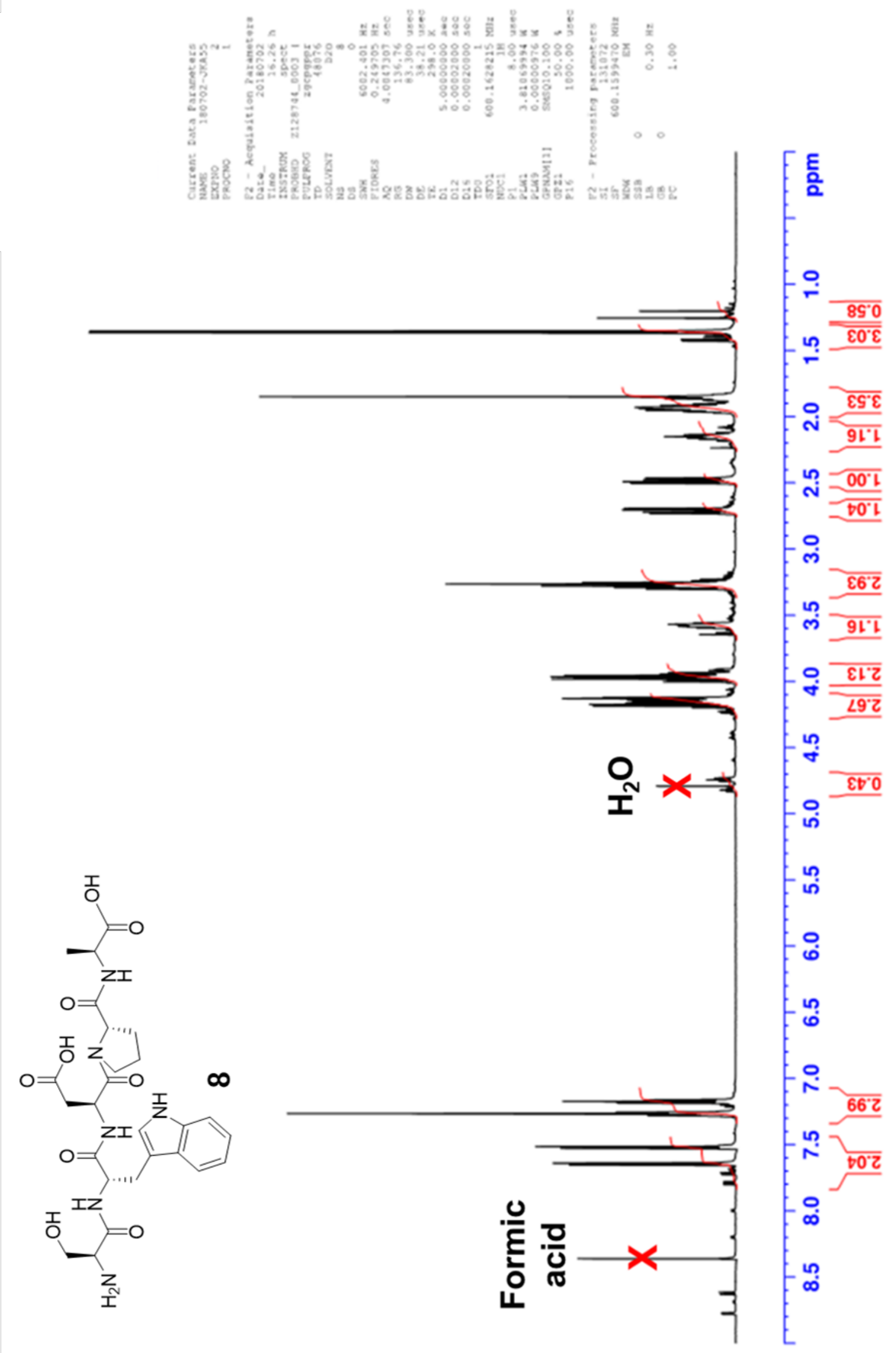




\section{${ }^{1} \mathrm{H}-{ }^{1} \mathrm{H}$ COSY of Compound 8}

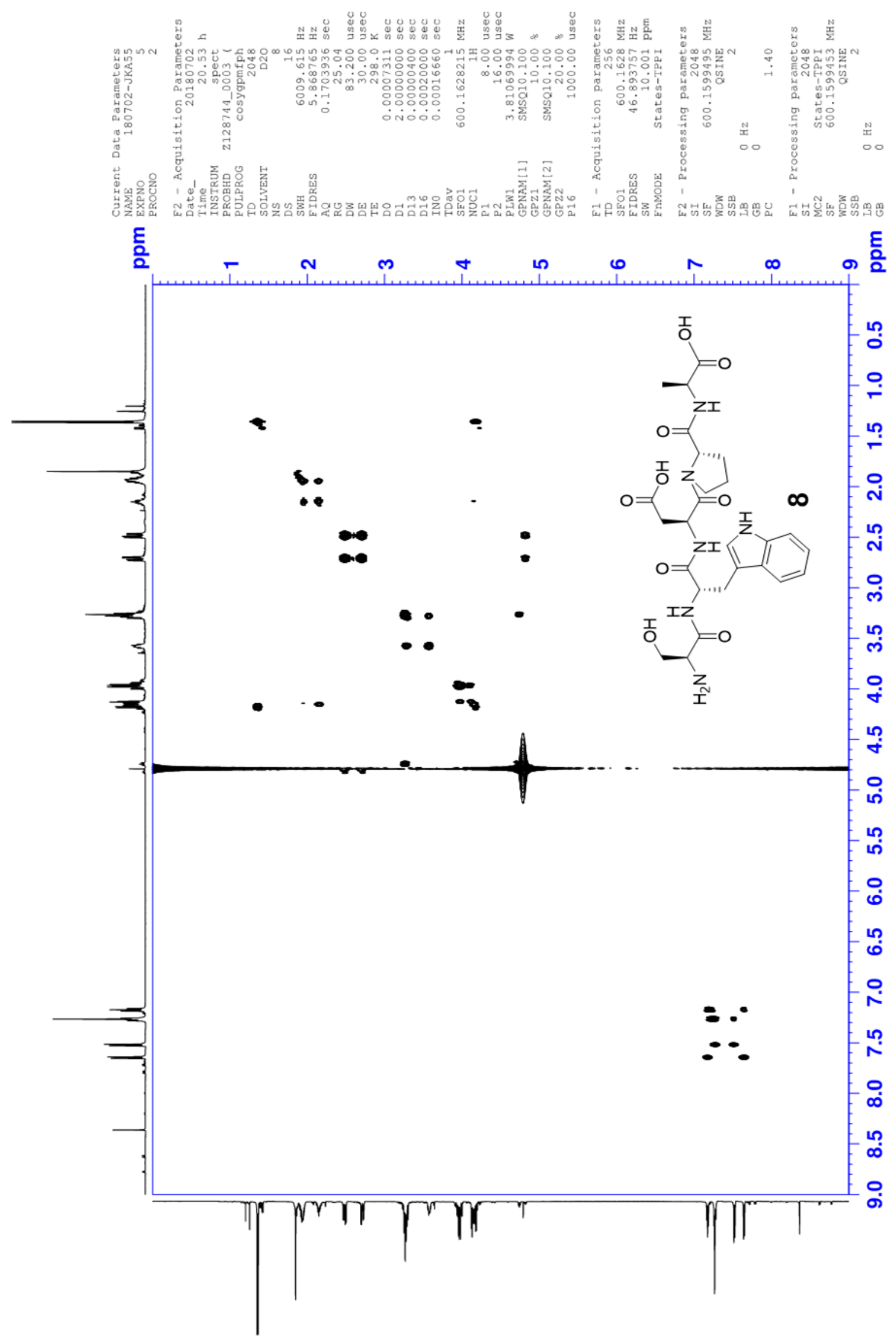




\section{${ }^{1} \mathrm{H}-{ }^{13} \mathrm{C}$ HSQC of Compound 8}

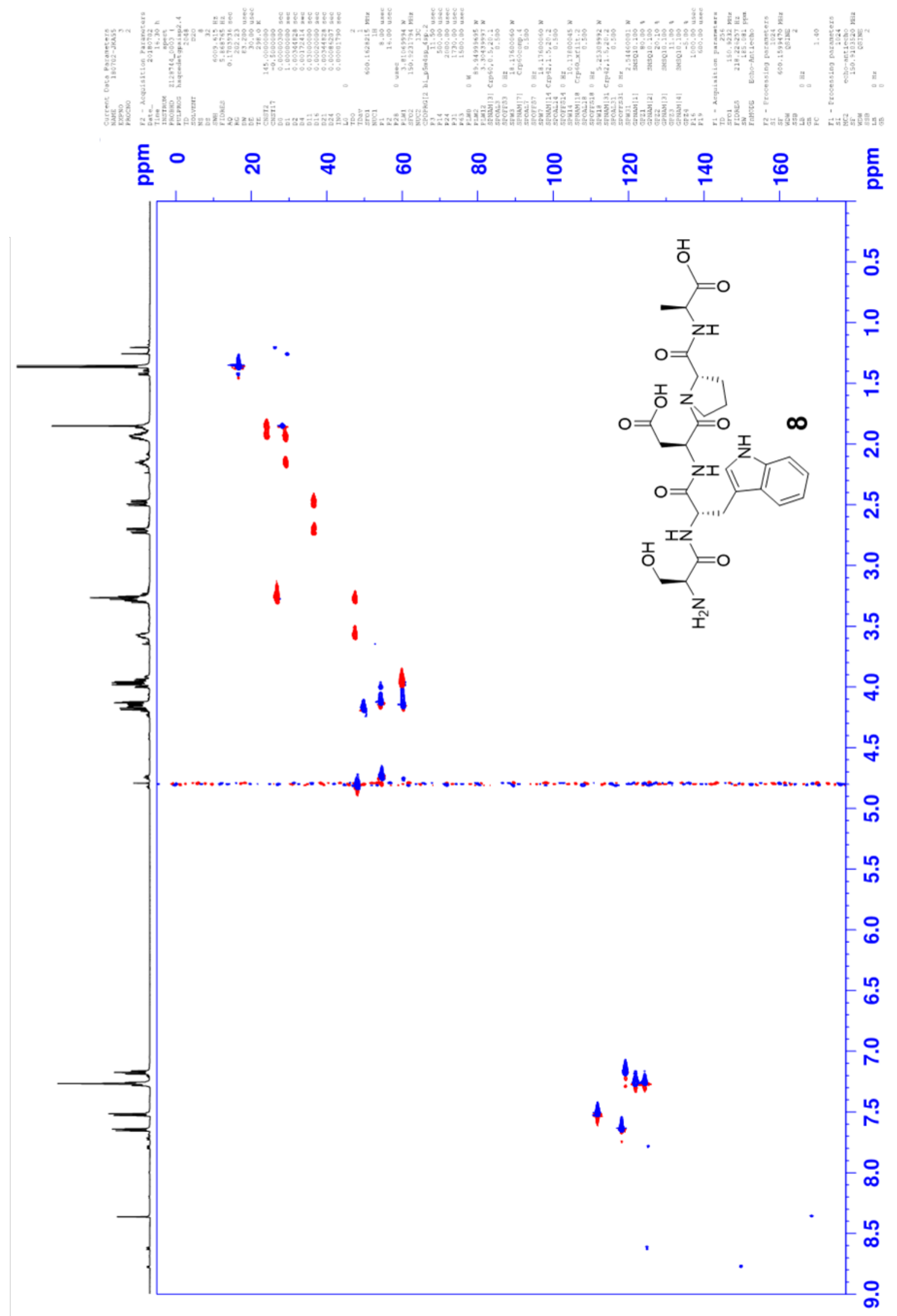




\section{${ }^{1} \mathrm{H}-{ }^{13} \mathrm{C}$ HMBC of Compound 8}

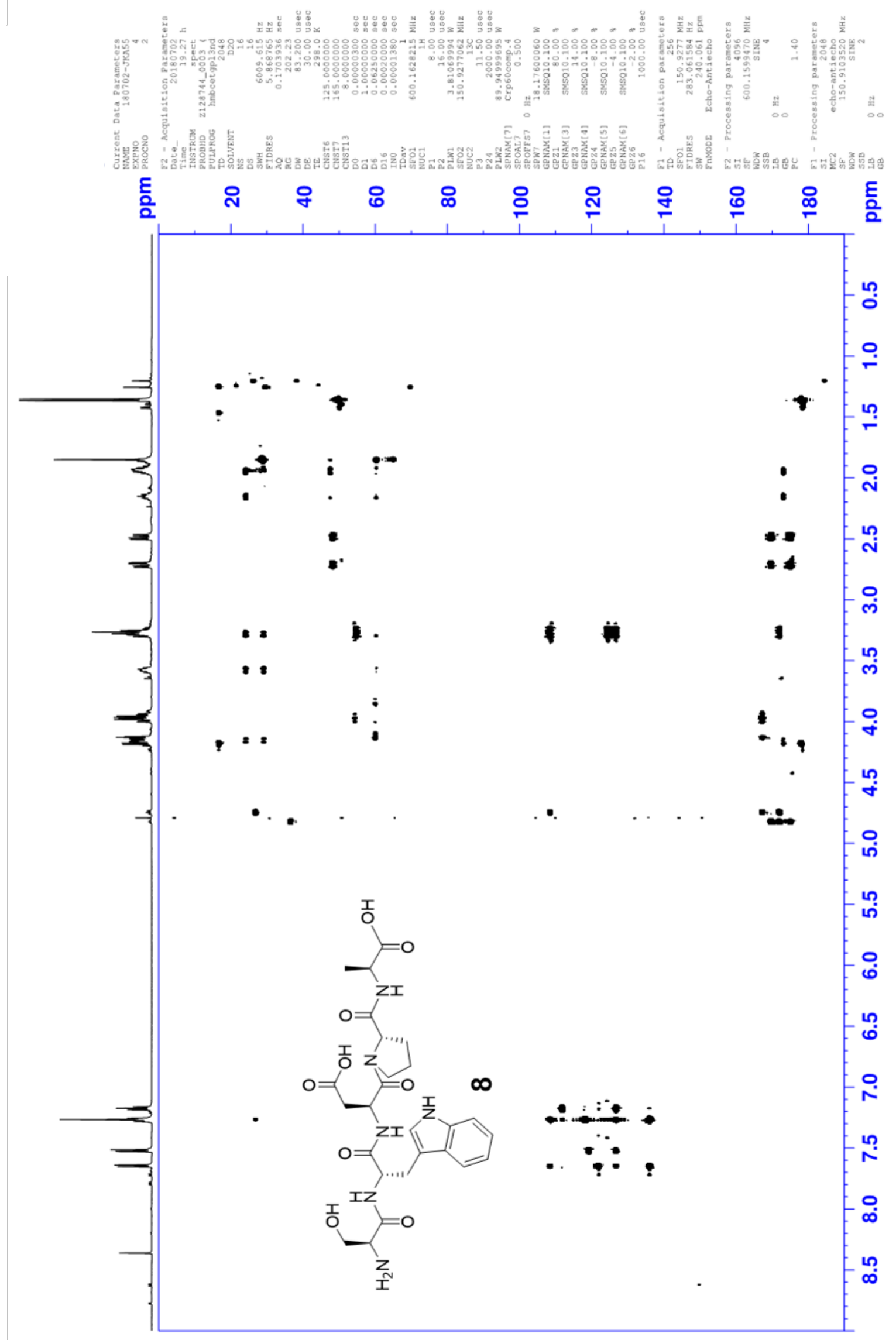




\section{LC/MS of Compound 9}<smiles>NC(CC(=O)O)C(=O)N[C@@H](Cc1c[nH]c2ccccc12)C(=O)N[C@@H](CC(=O)O)C(=O)N1CCC[C@H]1C(=O)N[C@@H](Cc1ccccc1)C(=O)O</smiles>

Chemical Formula: $\mathrm{C}_{33} \mathrm{H}_{38} \mathrm{~N}_{6} \mathrm{O}_{10}$ Exact Mass: 678.26

Molecular Weight: 678.70

mAU

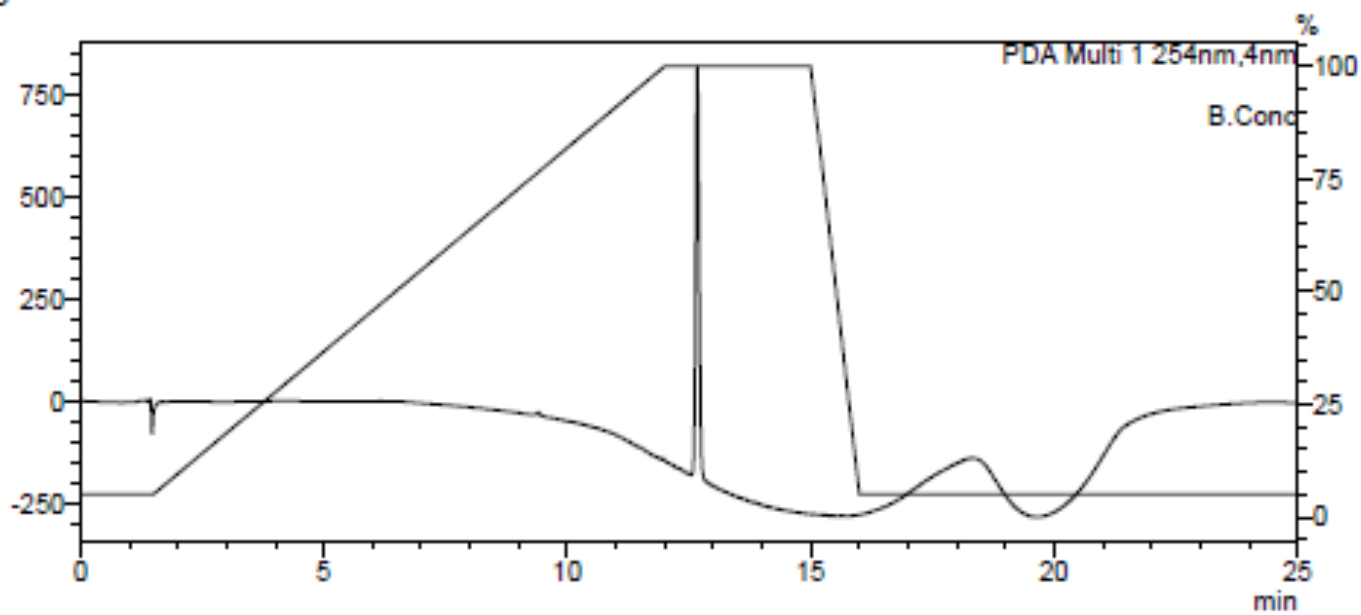

$(x 10,000,000)$

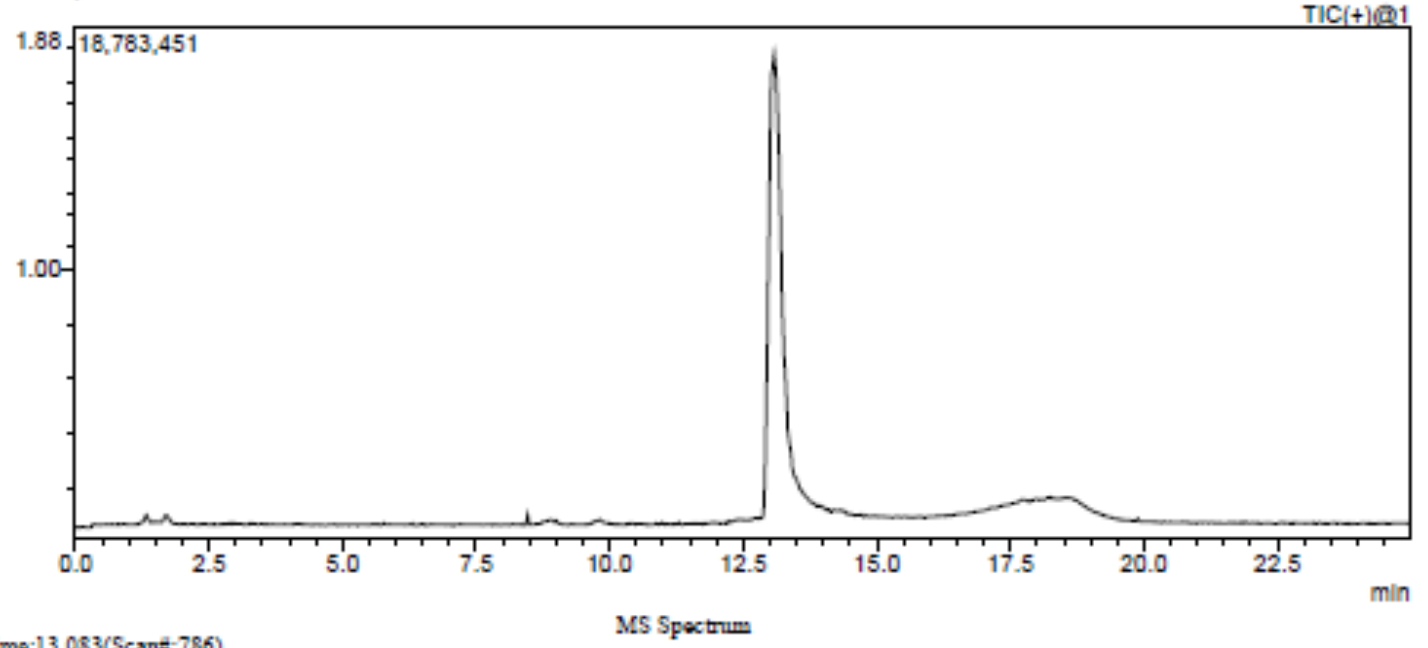

R. Time:13.083(Scans:786)

MS Spectrum

Sogment 1 - Evont

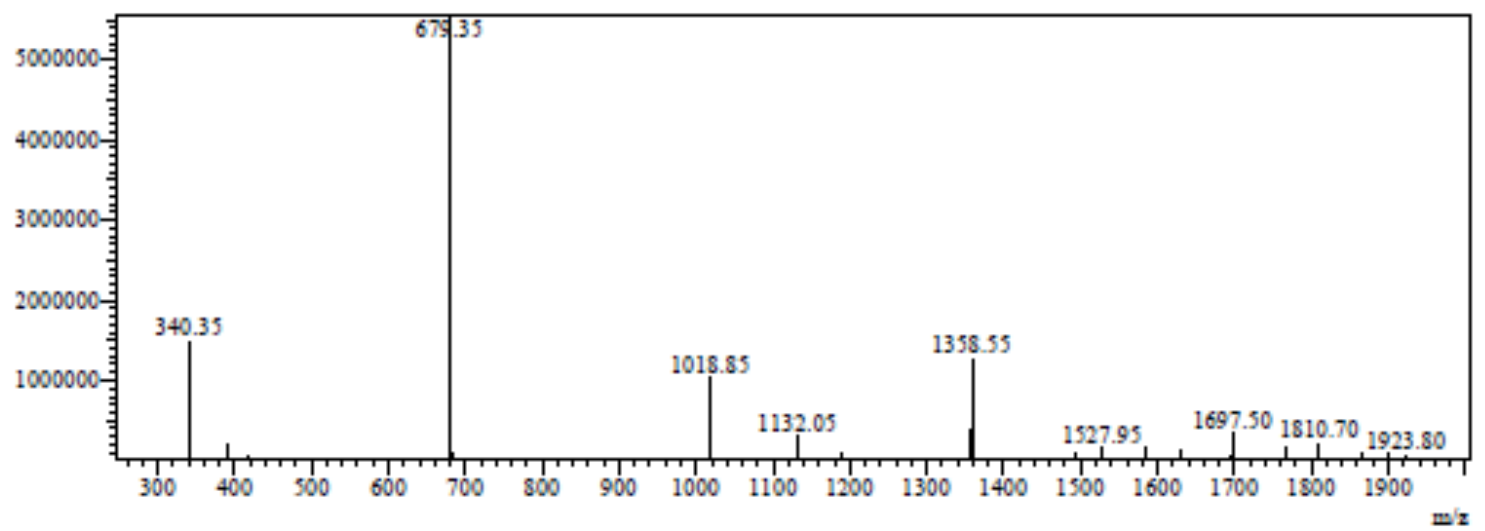




\section{${ }^{1} \mathrm{H}$ NMR of Compound 9}
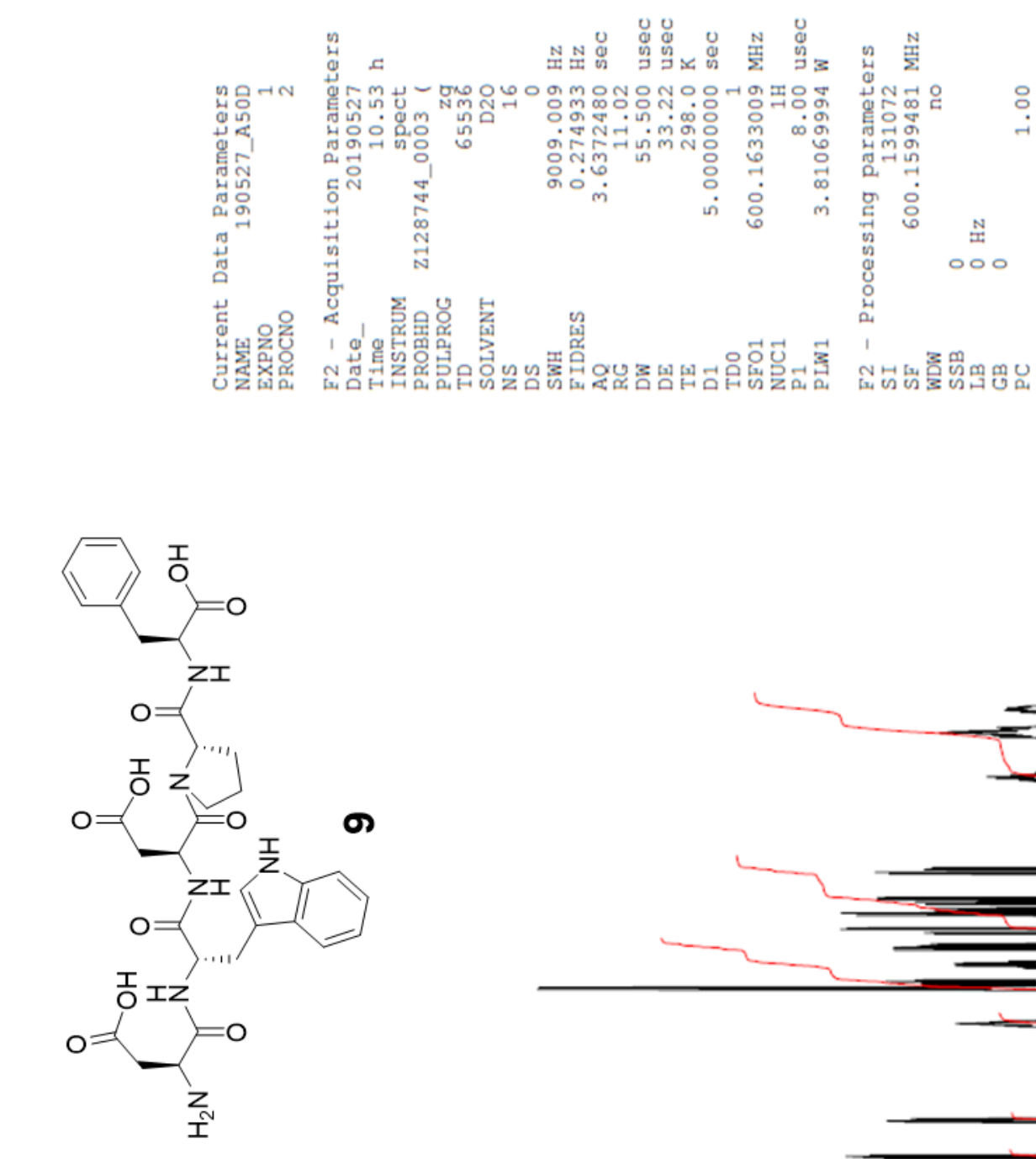

$\sigma$
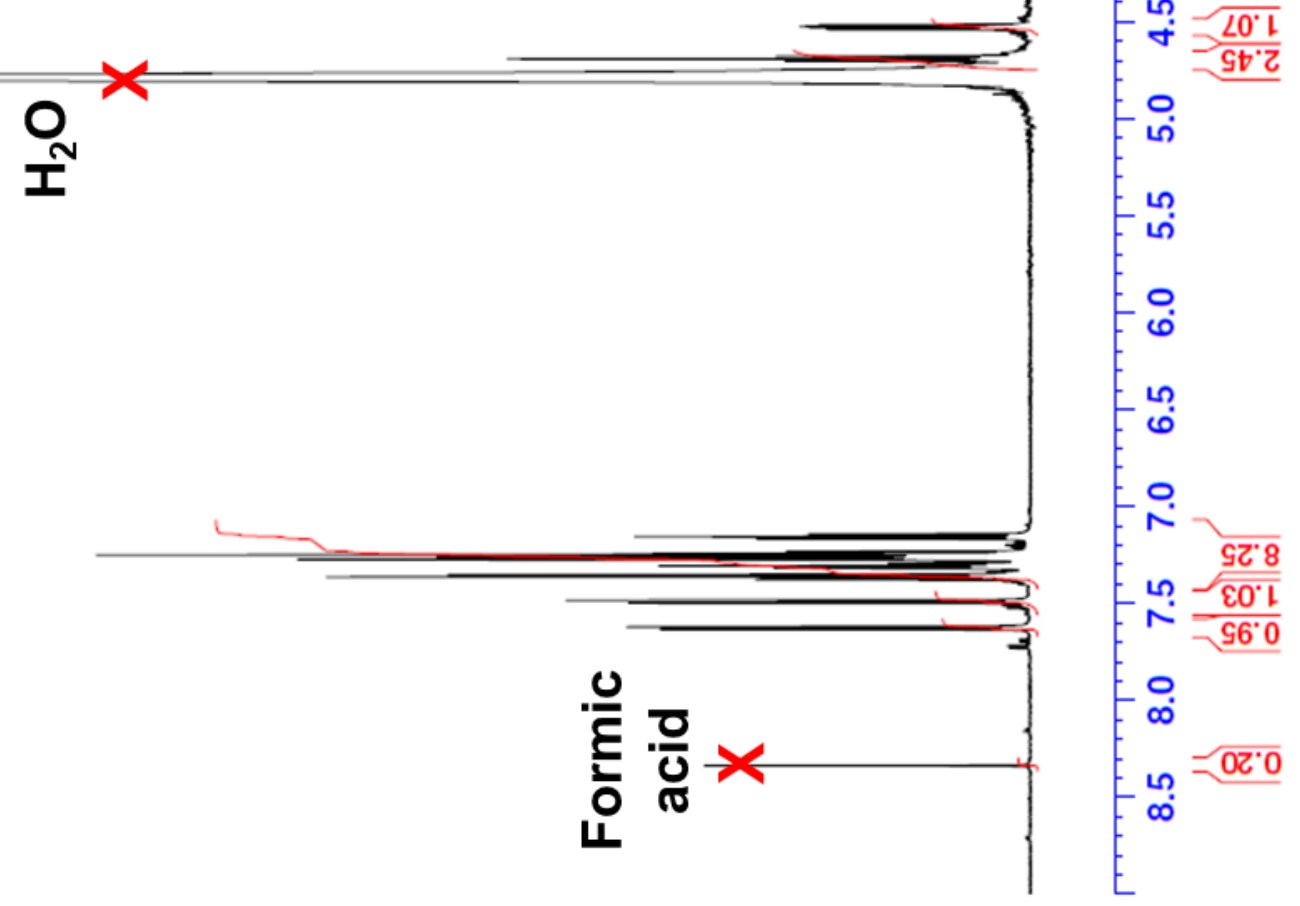


\section{${ }^{1} \mathrm{H}-{ }^{1} \mathrm{H}$ COSY of Compound 9}
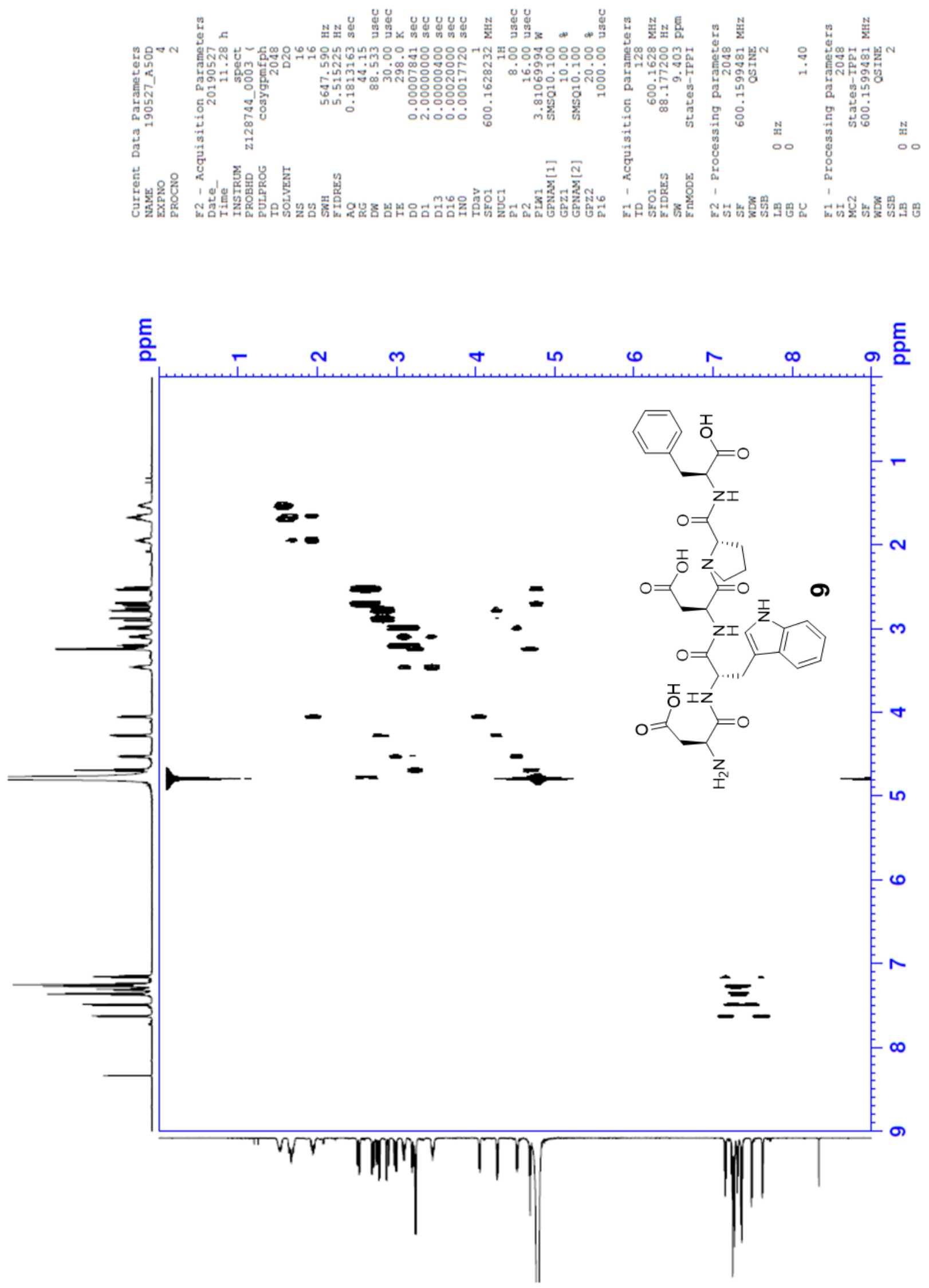


\section{${ }^{1} \mathrm{H}-{ }^{13} \mathrm{C}$ HSQC of Compound 9}
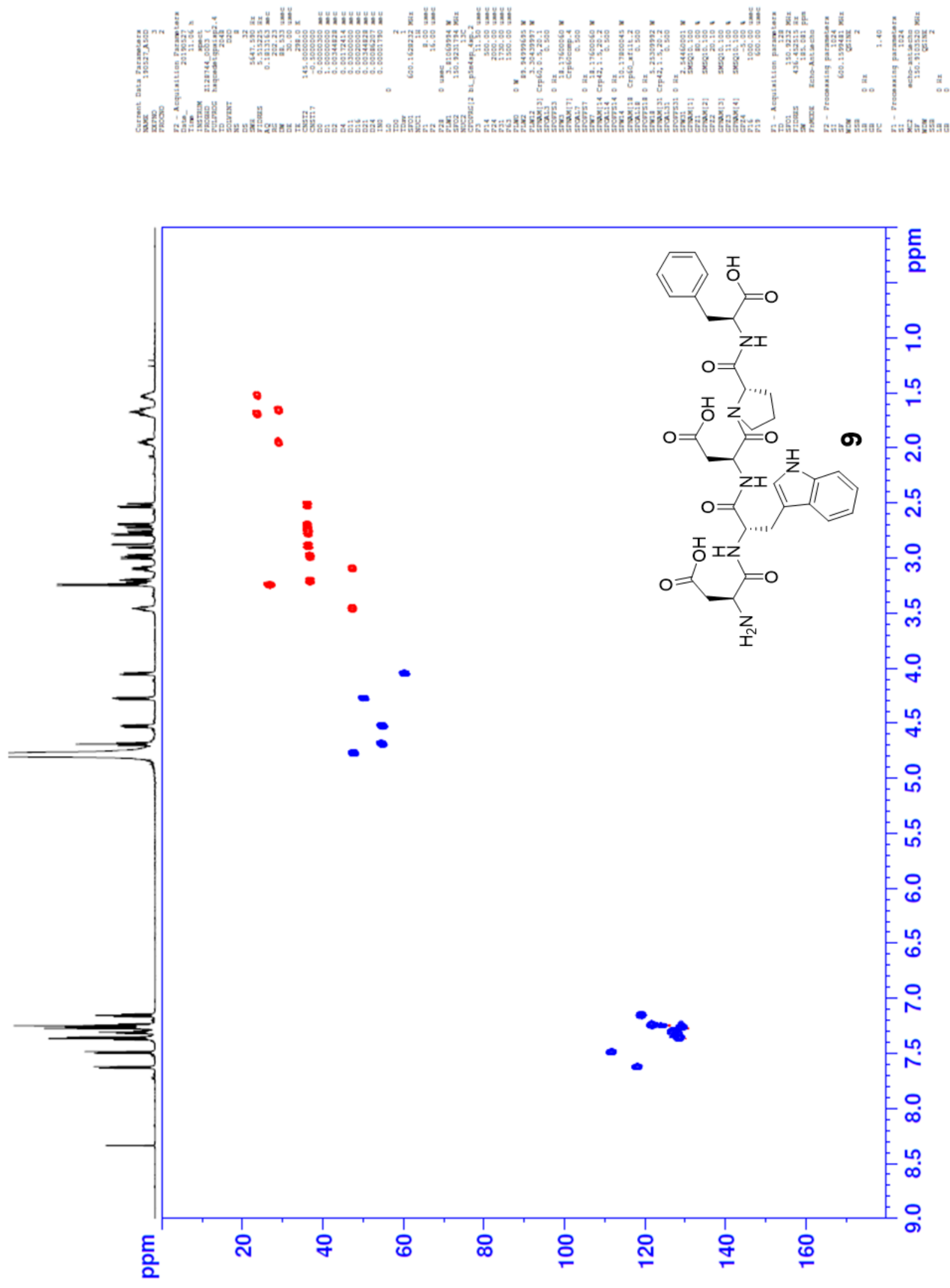


\section{${ }^{1} \mathrm{H}-{ }^{13} \mathrm{C}$ HMBC of Compound 9}
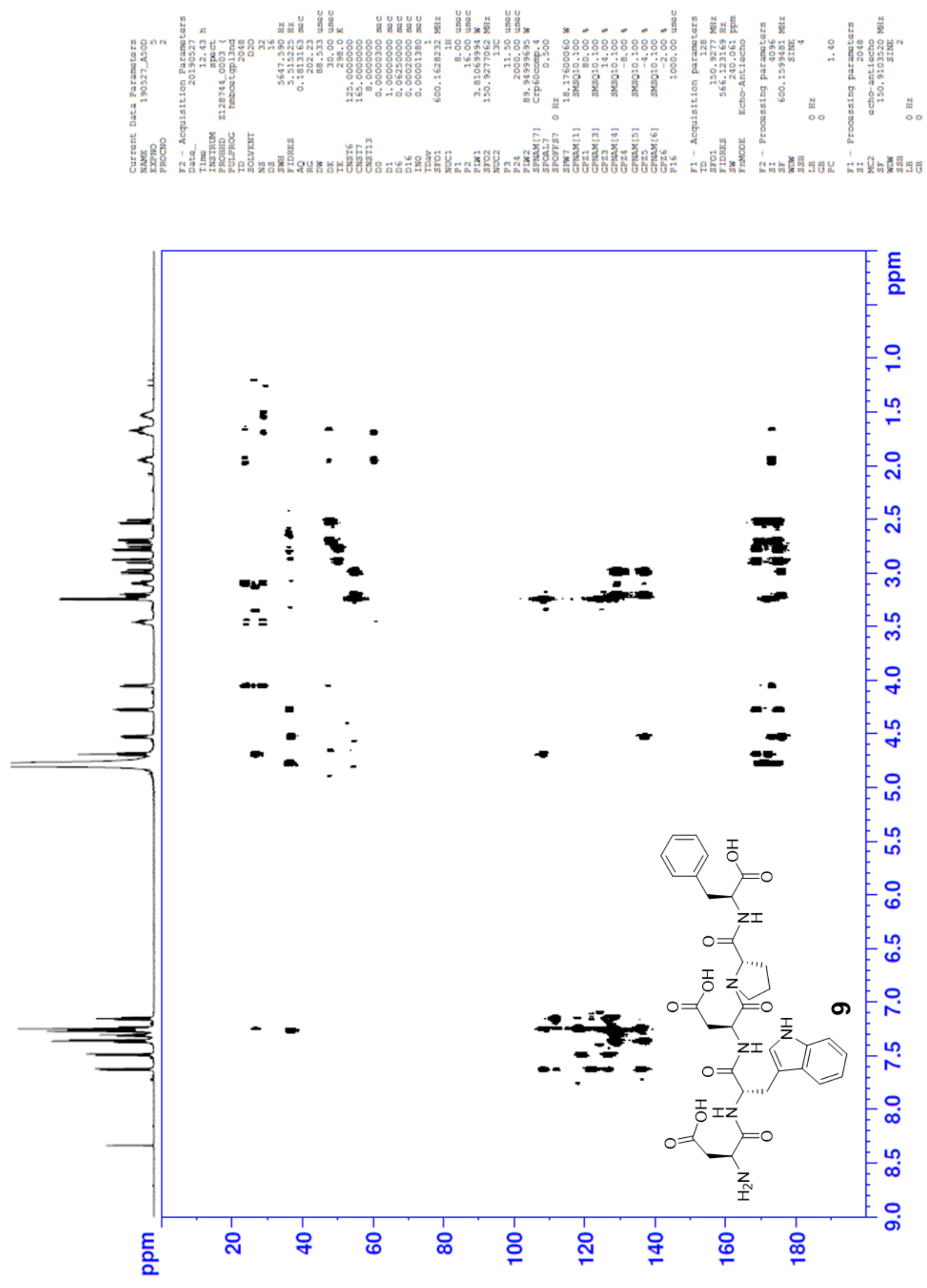


\section{LC/MS of Compound 10}<smiles>N[C@@H](CO)C(=O)N[C@@H](Cc1c[nH]c2ccccc12)C(=O)N[C@@H](CC(=O)O)C(=O)N1CCC[C@H]1C(=O)N[C@@H](Cc1ccccc1)C(=O)O</smiles>

Chemical Formula: $\mathrm{C}_{32} \mathrm{H}_{38} \mathrm{~N}_{6} \mathrm{O}_{9}$

Exact Mass: 650.27

Molecular Weight: 650.69

mAU

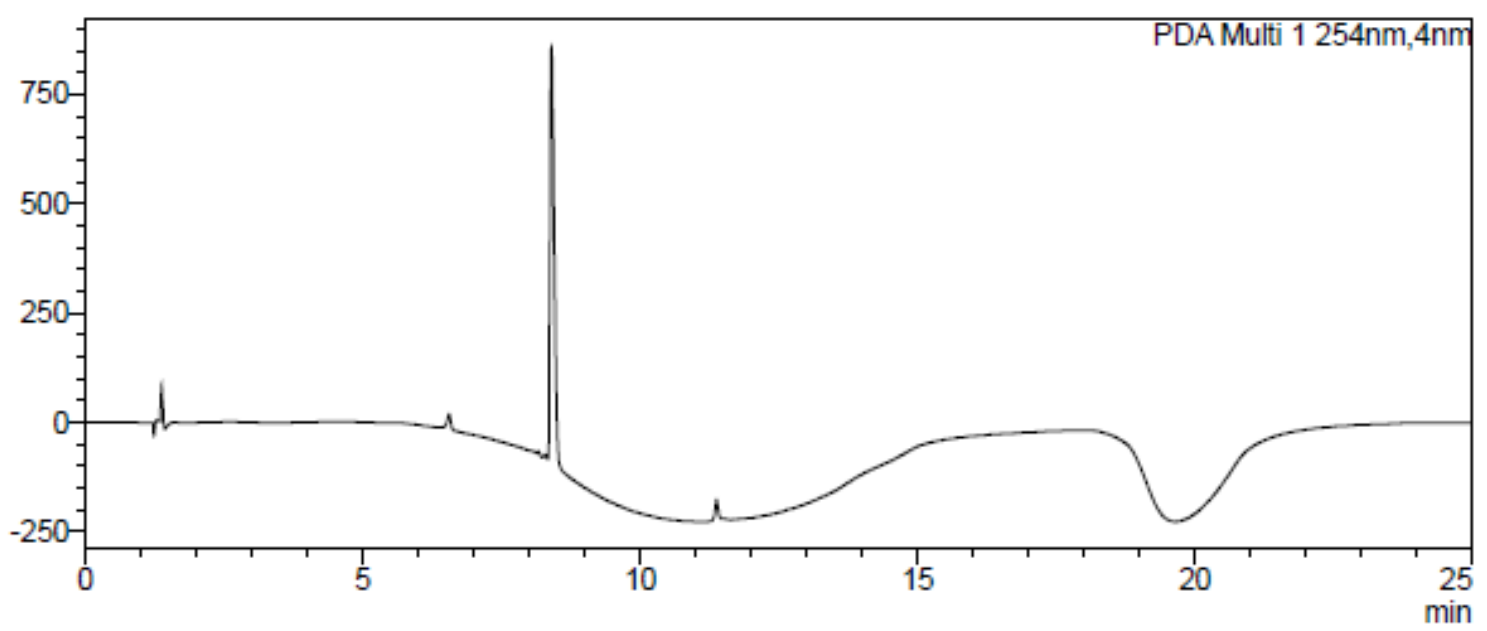

$(\mathrm{x} 10,000,000)$

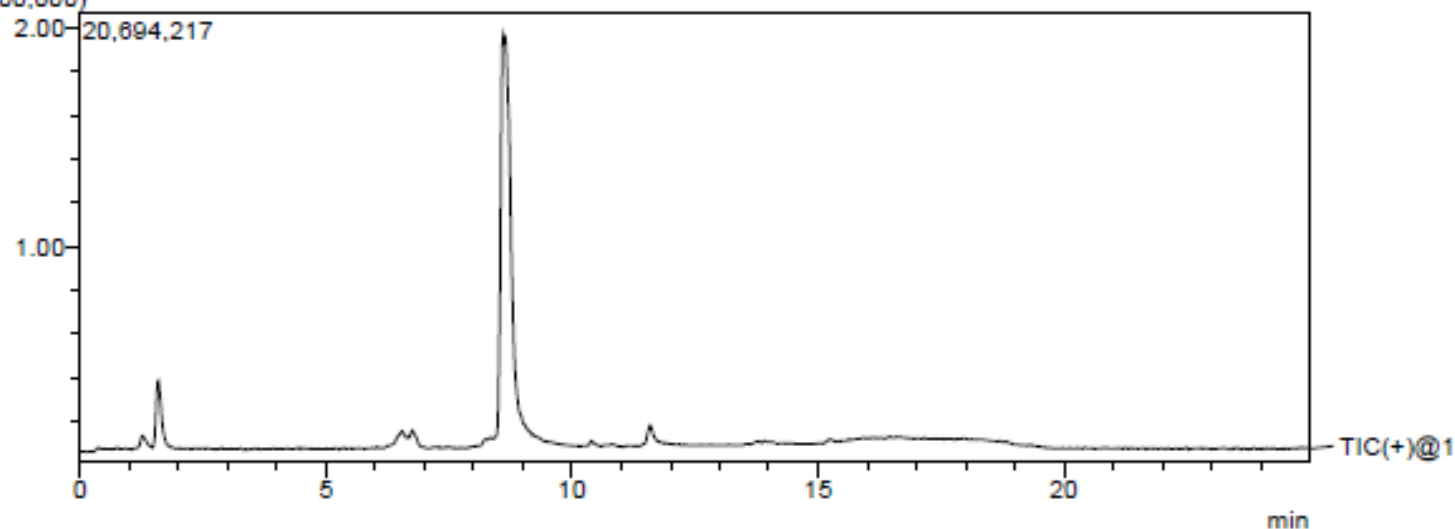

MS Spectrum

R. Time: 8.633 (Scan $\# .519$ )

MassPealks: 1900

Segment 1 - Event 1

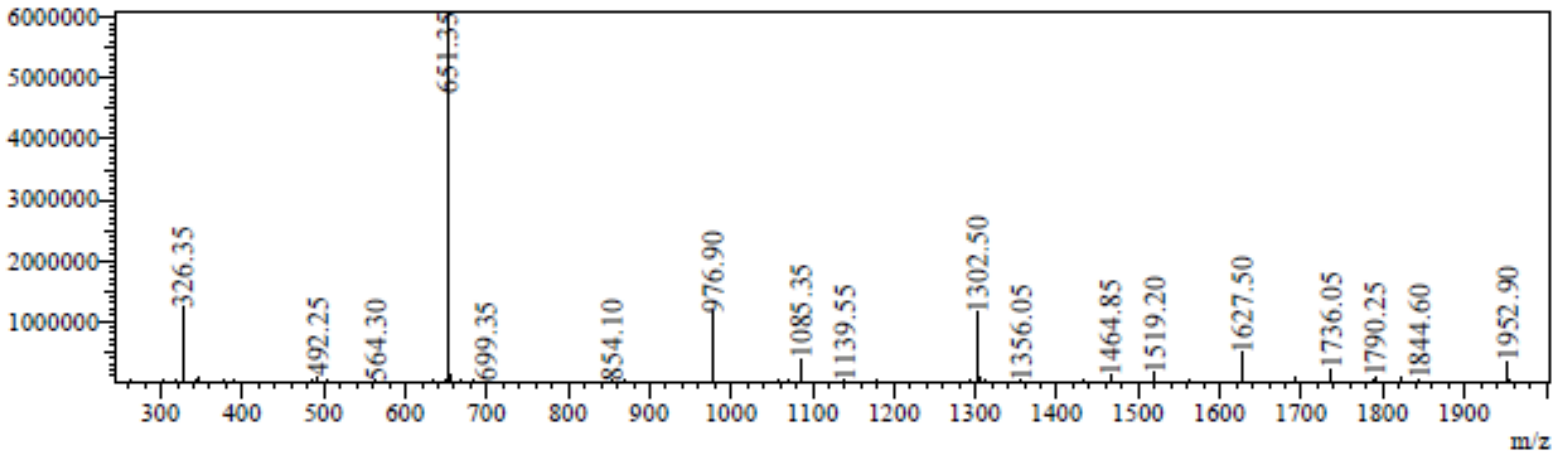




\section{${ }^{1} \mathrm{H}$ NMR of Compound 10}
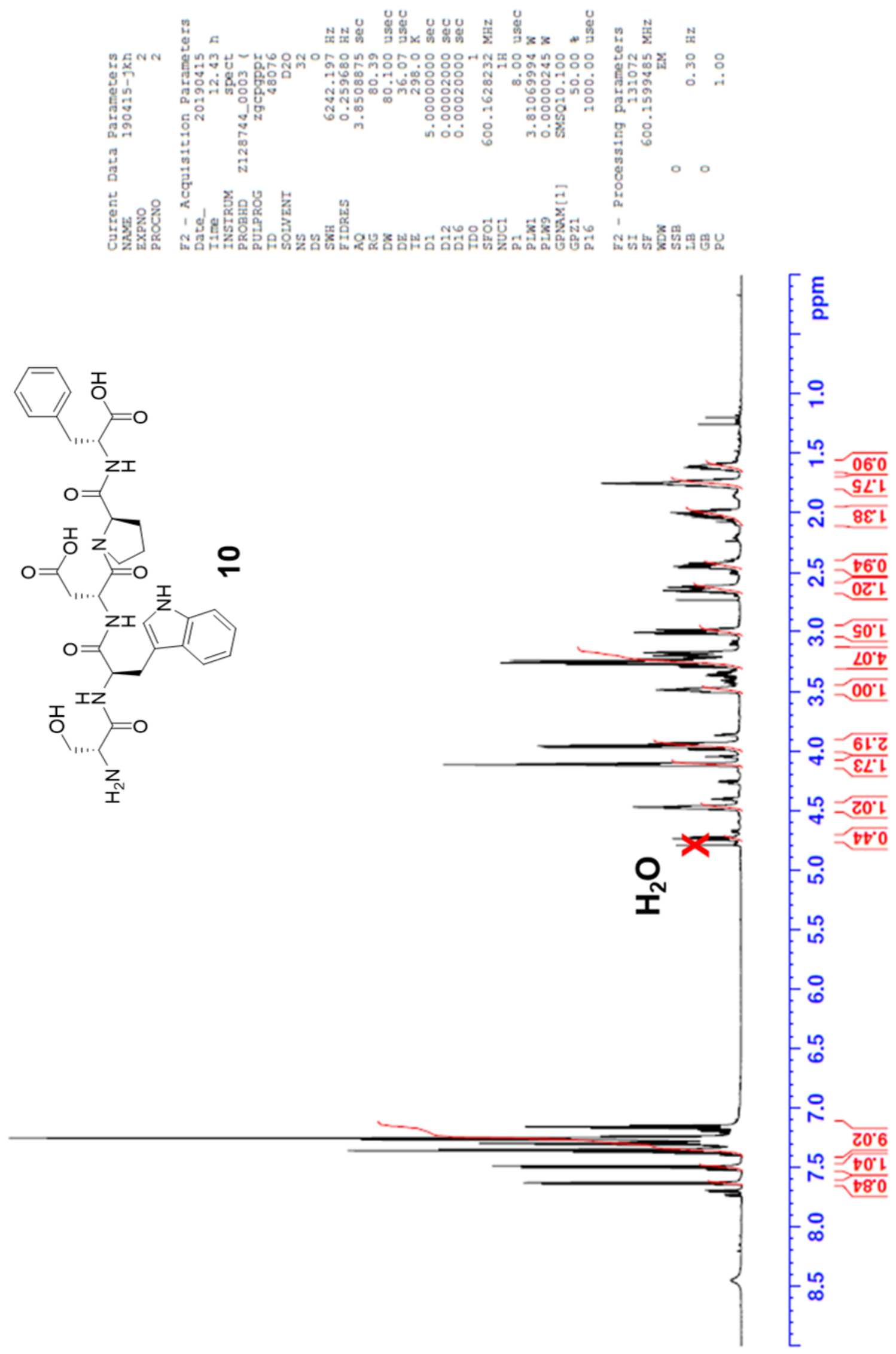


\section{${ }^{1} \mathrm{H}-{ }^{1} \mathrm{H}$ COSY of Compound 10}

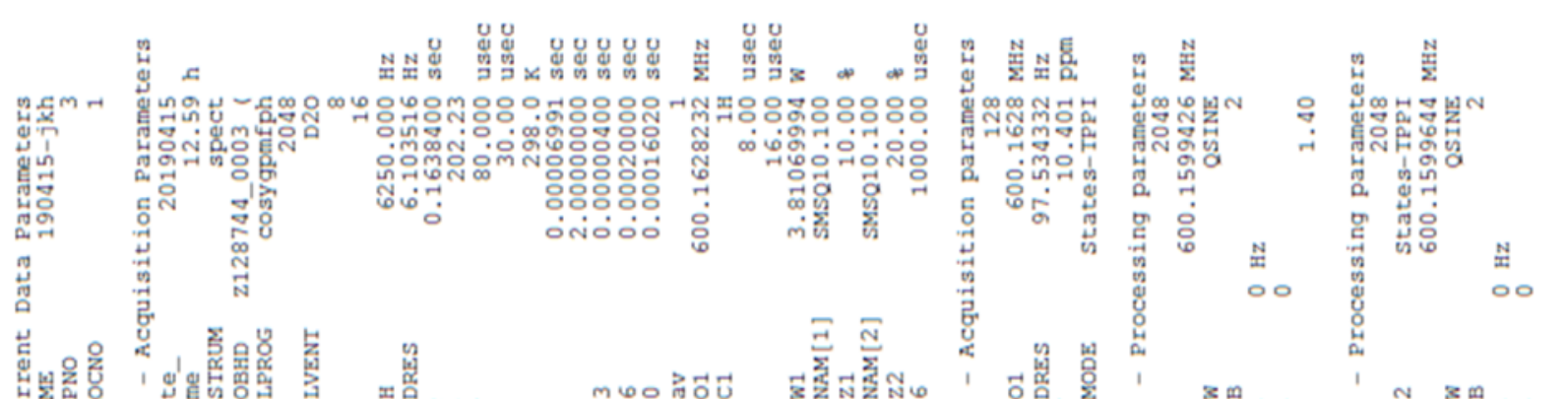

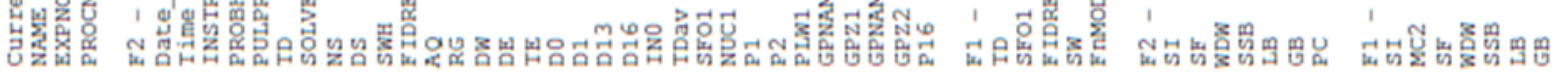

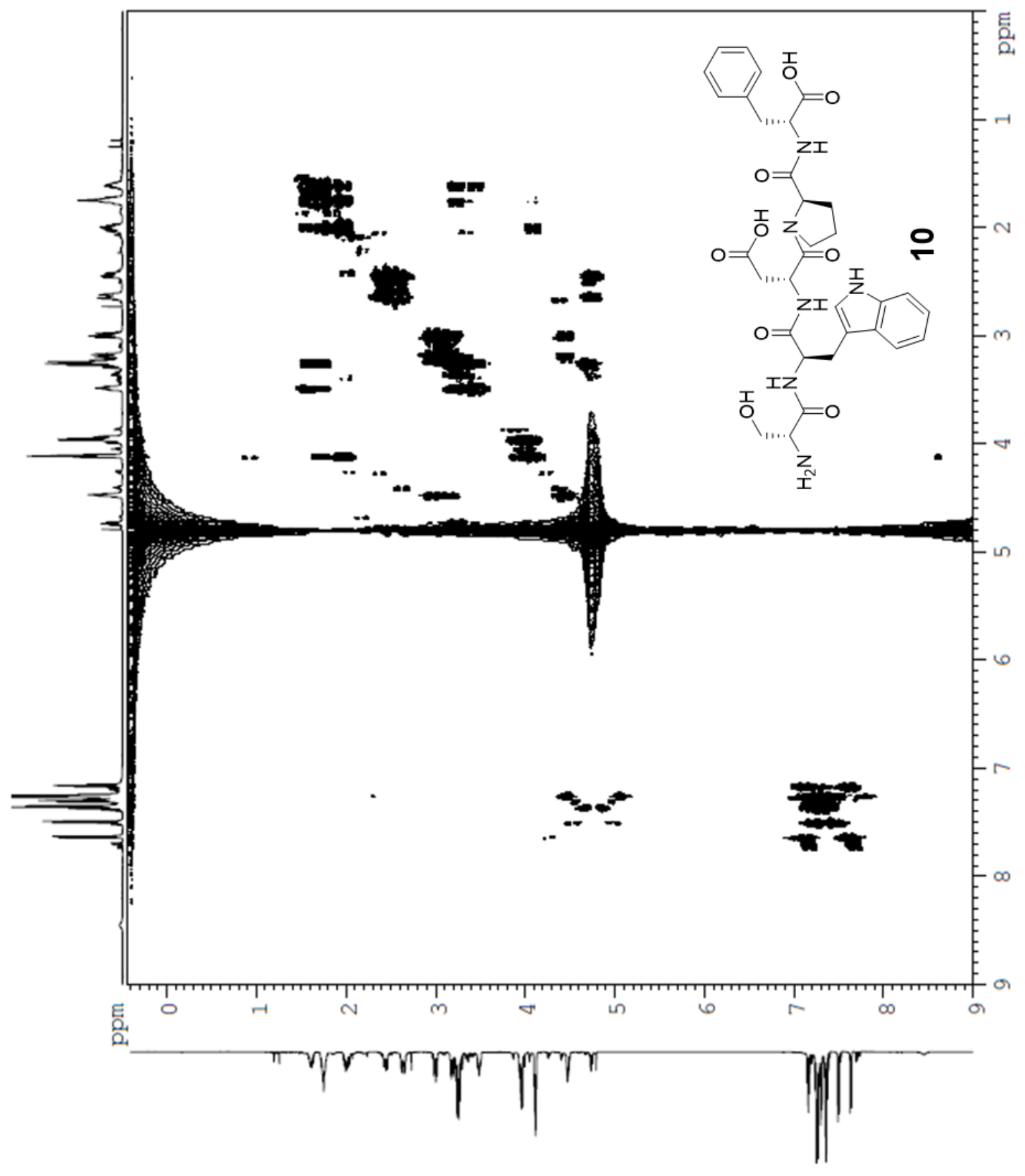




\section{${ }^{1} \mathrm{H}-{ }^{13} \mathrm{C}$ HSQC of Compound 10}
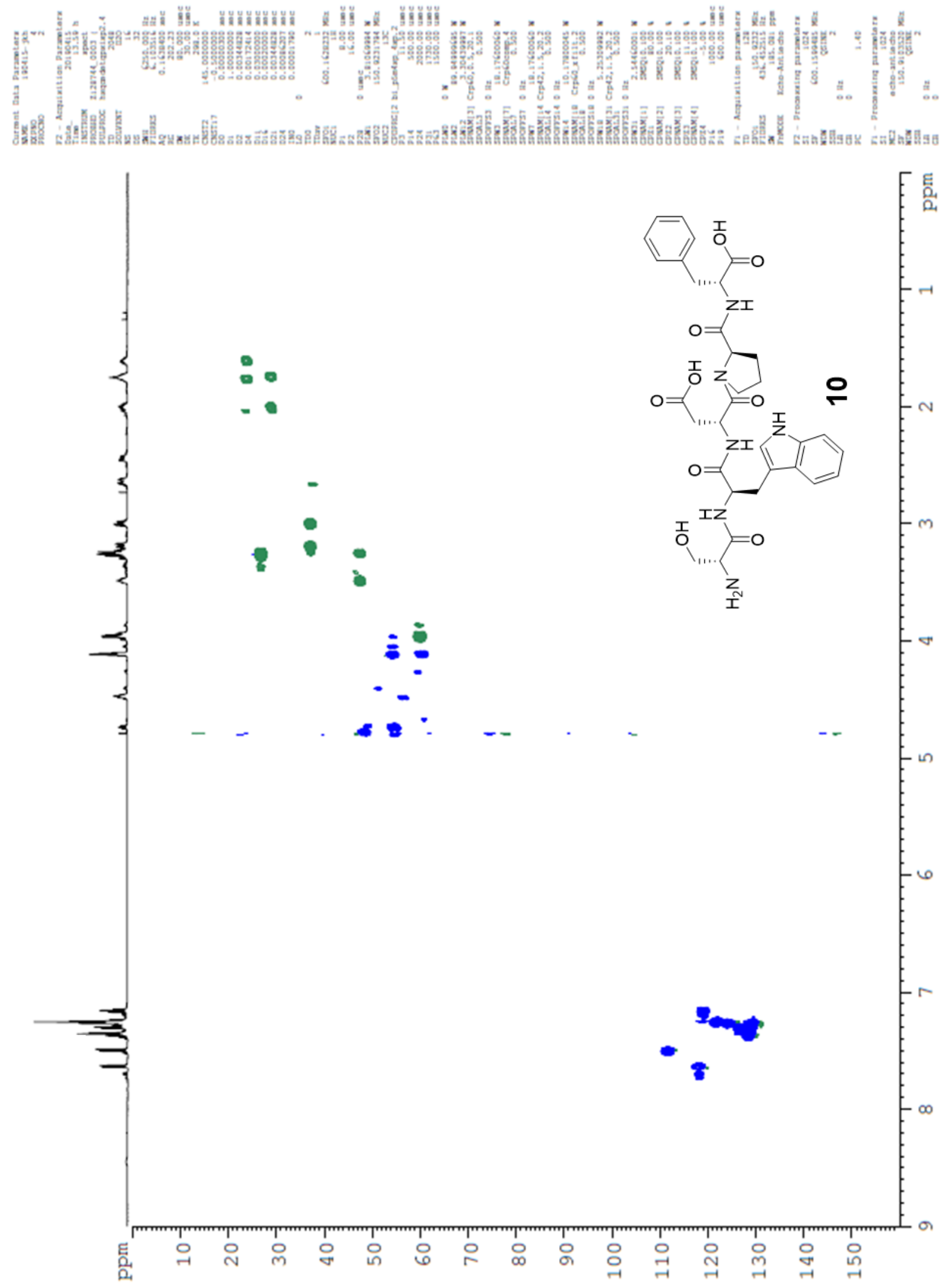


\section{${ }^{1} \mathrm{H}-{ }^{13} \mathrm{C}$ HMBC of Compound 10}

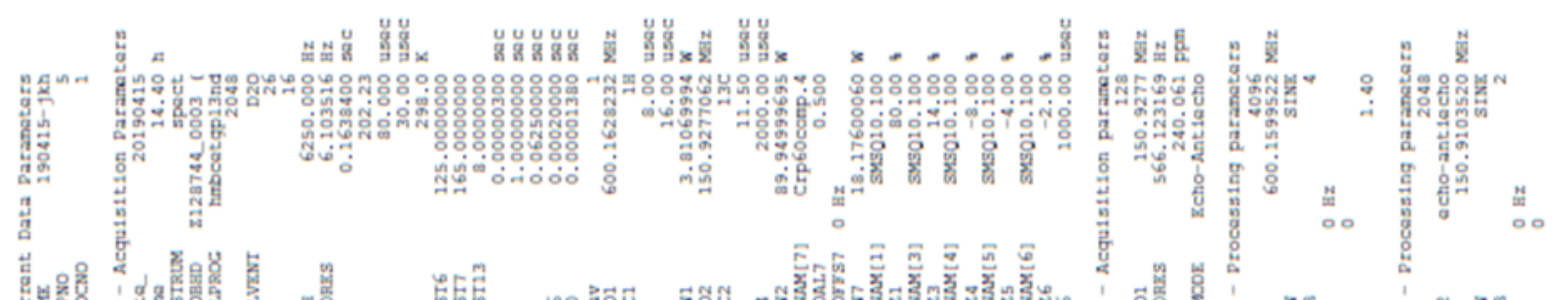

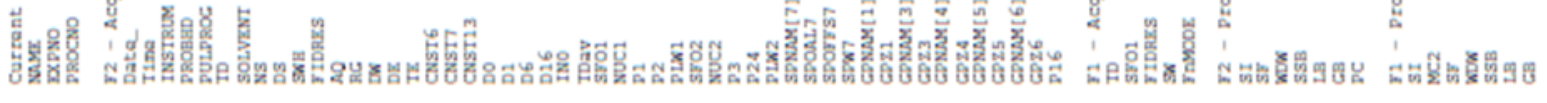

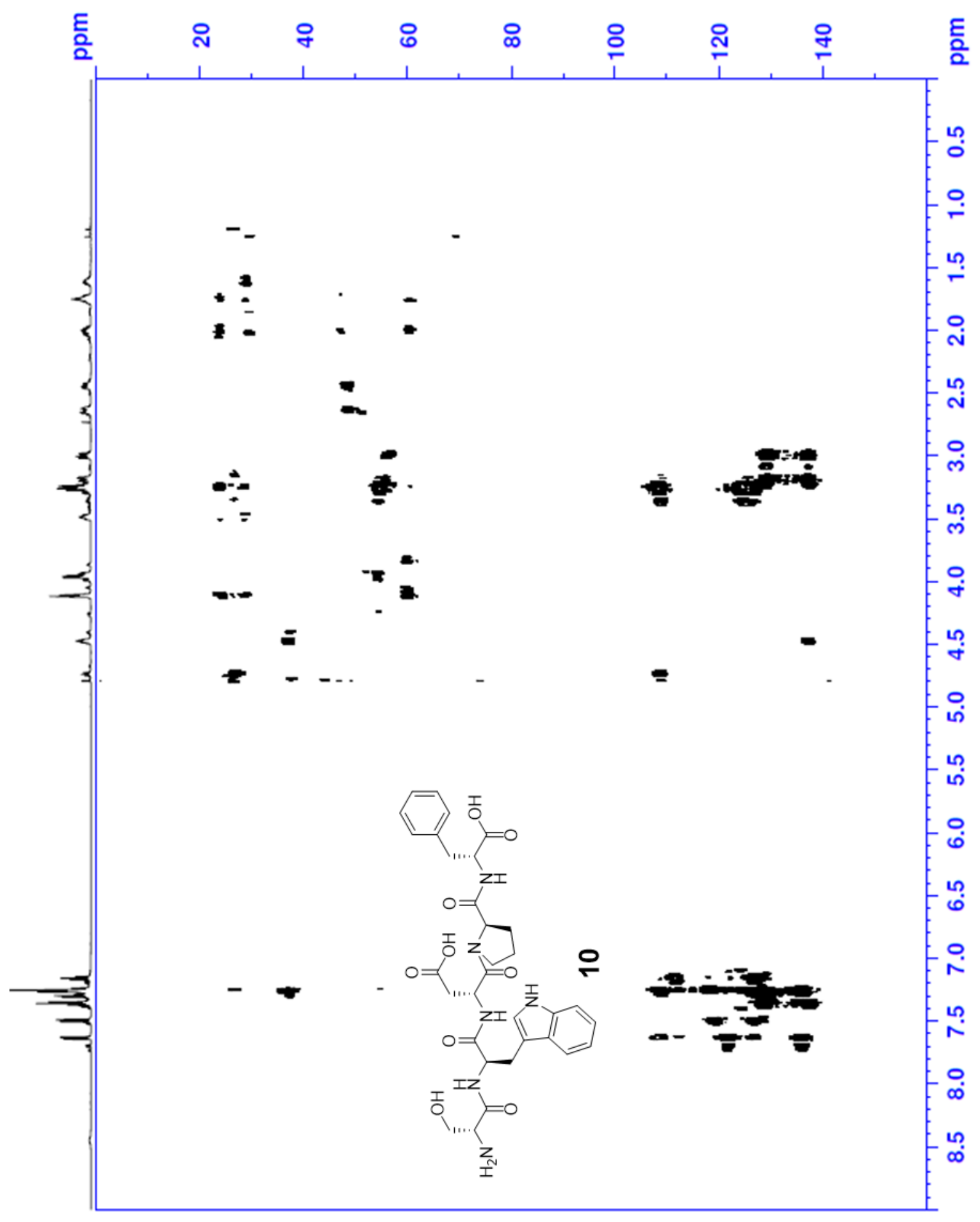




\section{LC/MS of Compound 11}<smiles>N=C(N)NCCC[C@H](NC(=O)C(Cc1ccccc1)NC(=O)C1CCC(C(CC(=O)O)NC(=O)[C@H](Cc2c[nH]c3ccccc23)NC(=O)C(N)CO)N1)C(=O)O</smiles>

Chemical Formula: $\mathrm{C}_{38} \mathrm{H}_{50} \mathrm{~N}_{10} \mathrm{O}_{10}$ Exact Mass: 806.37

Molecular Weight: 806.88

mAU

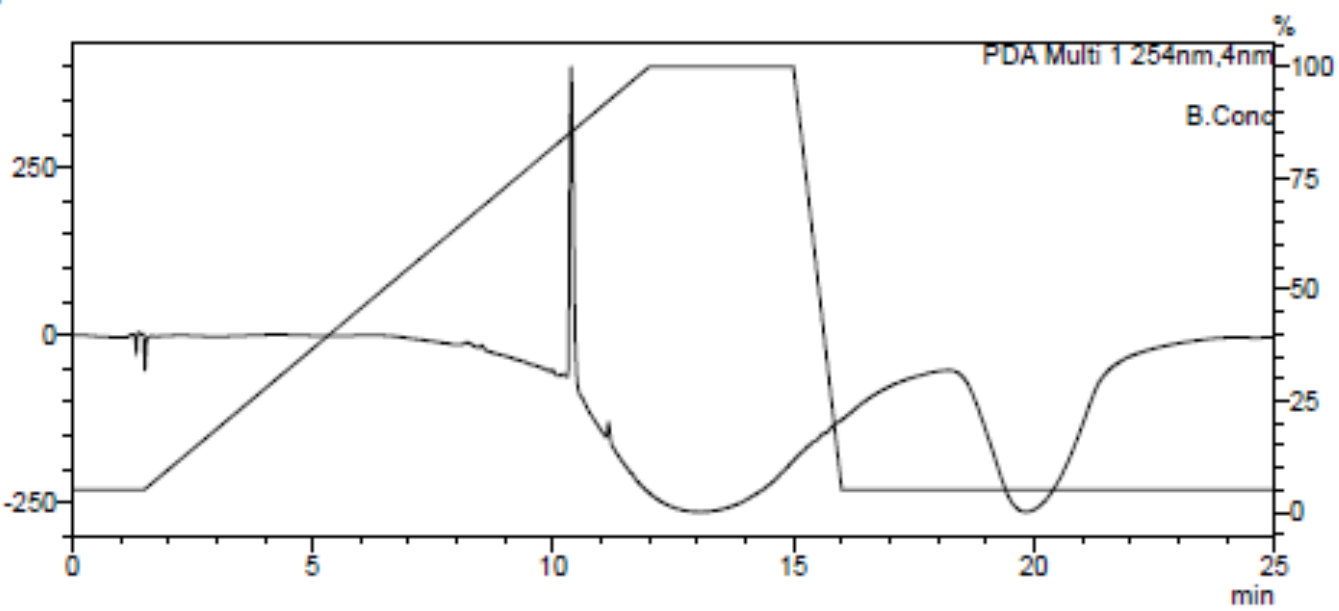

(x10,000,000)

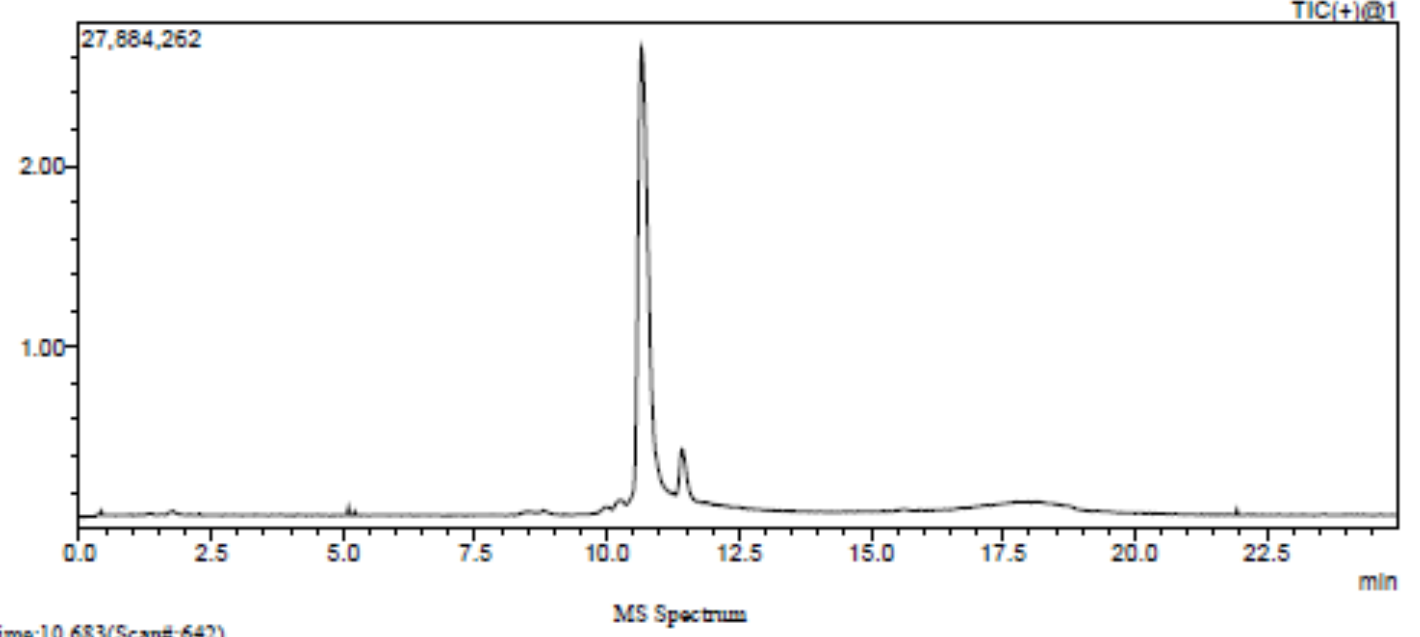

R. Time: 10.683 (Scans: 642 )

ass Peaks: 1938

Sogmeut 1 - Evont 1

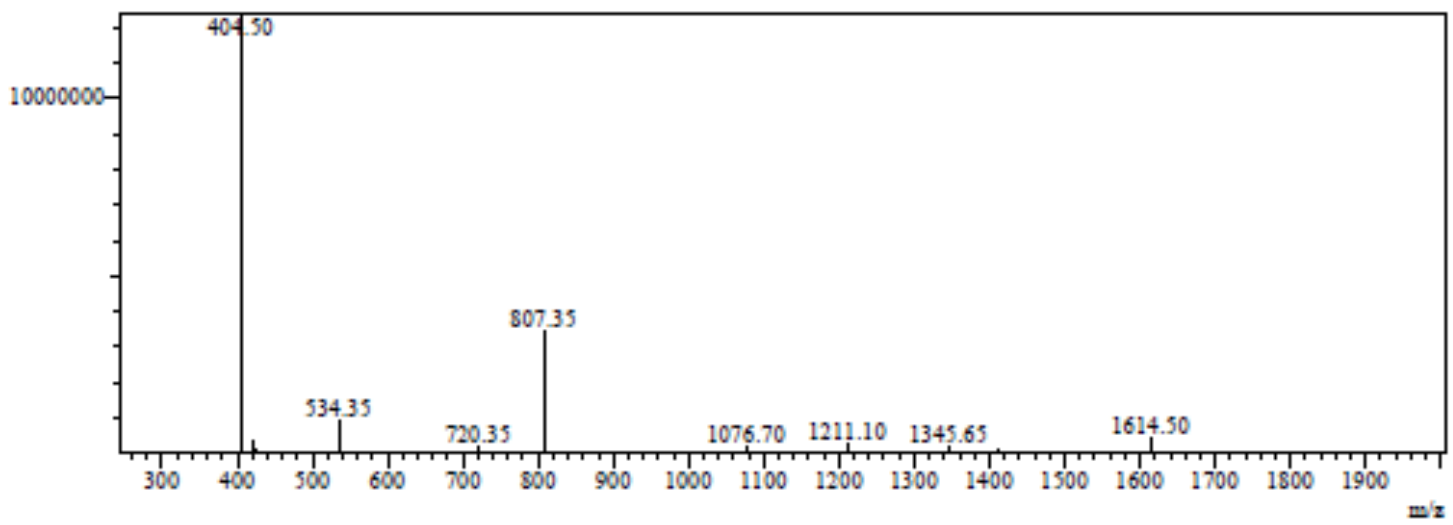




\section{${ }^{1} \mathrm{H}$ NMR of Compound 11}
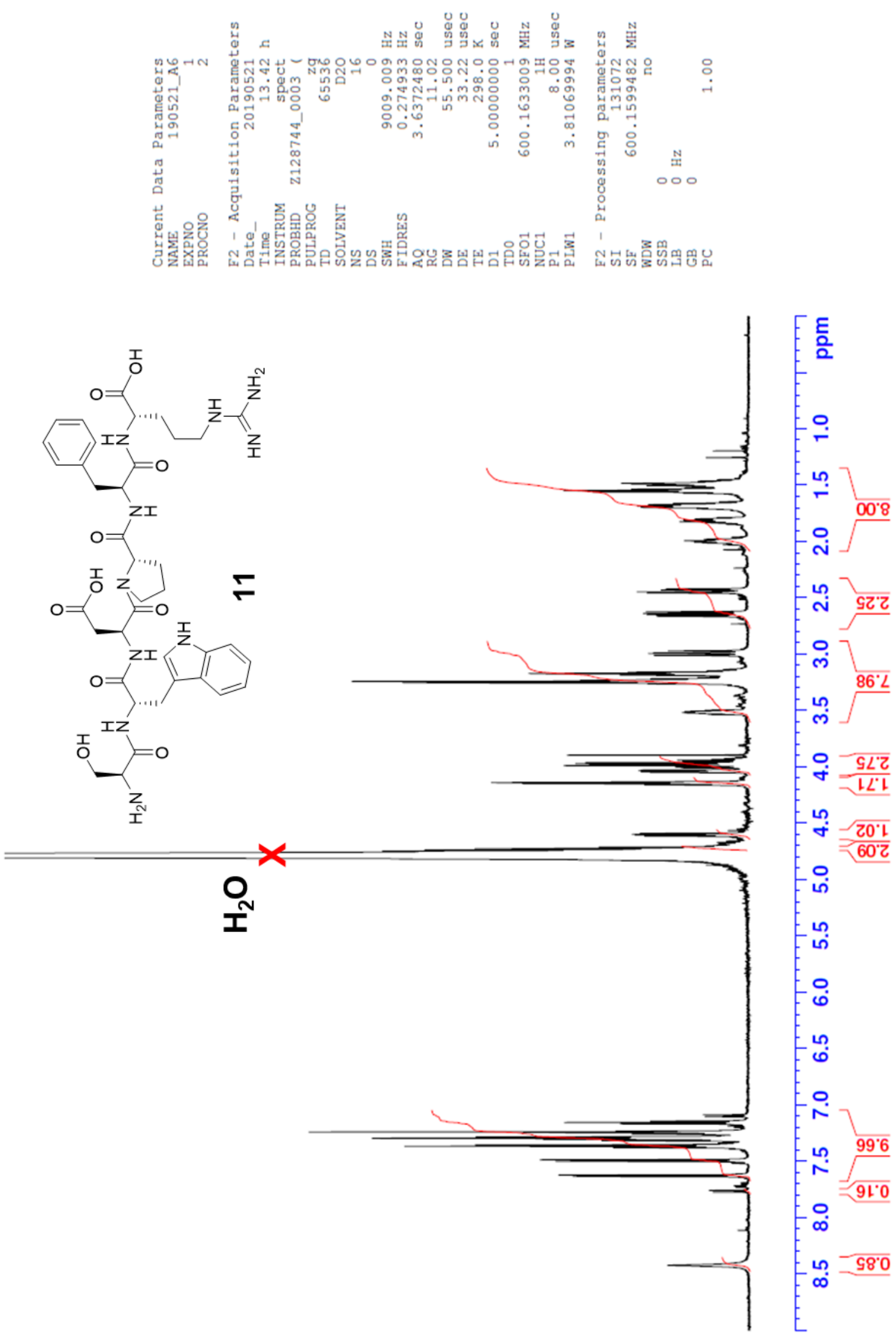


\section{${ }^{1} \mathrm{H}-{ }^{1} \mathrm{H}$ COSY of Compound 11}
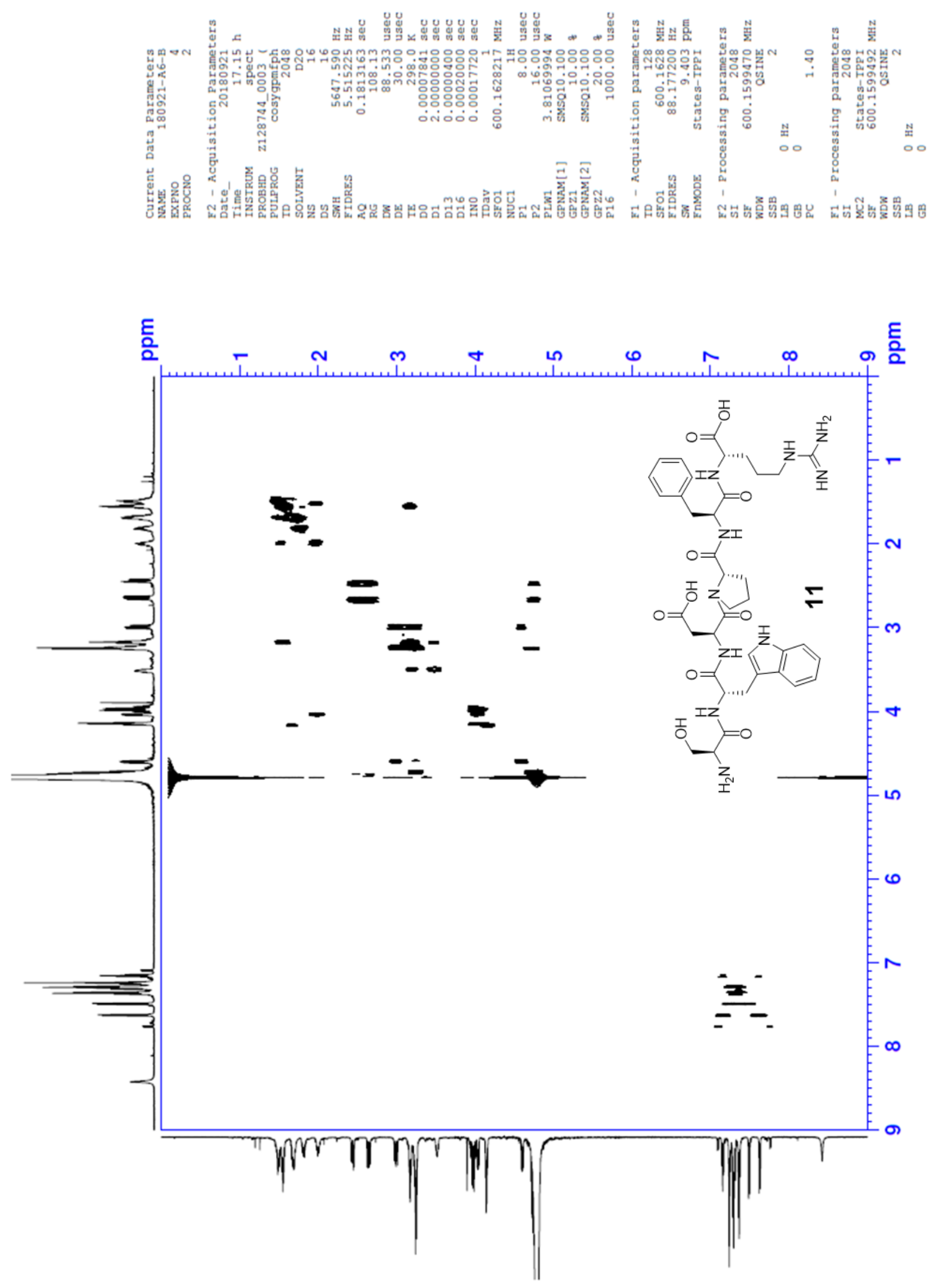


\section{${ }^{1} \mathrm{H}-{ }^{13} \mathrm{C}$ HSQC of Compound 11}

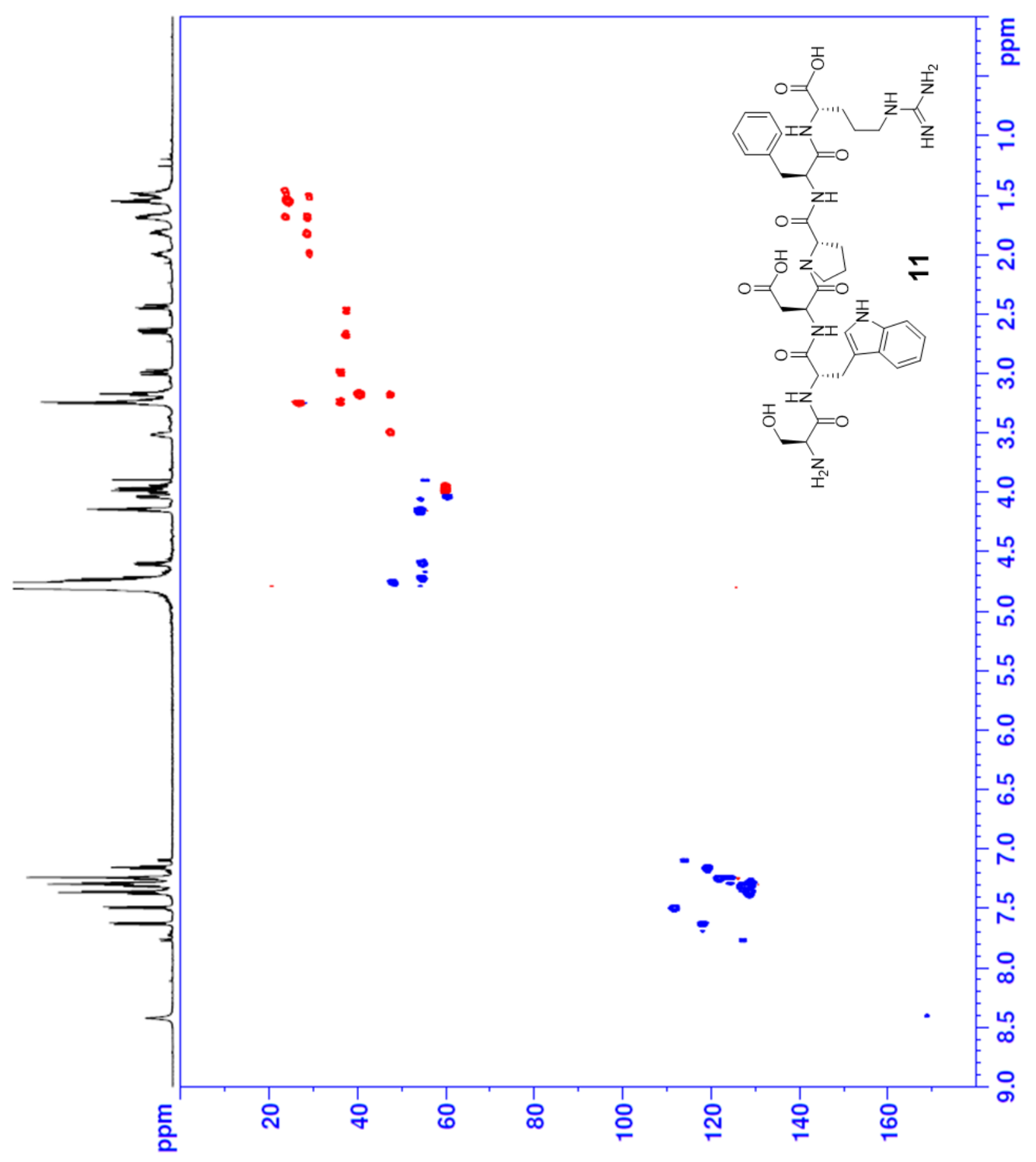




\section{${ }^{1} \mathrm{H}-{ }^{13} \mathrm{C}$ HMBC of Compound 11}
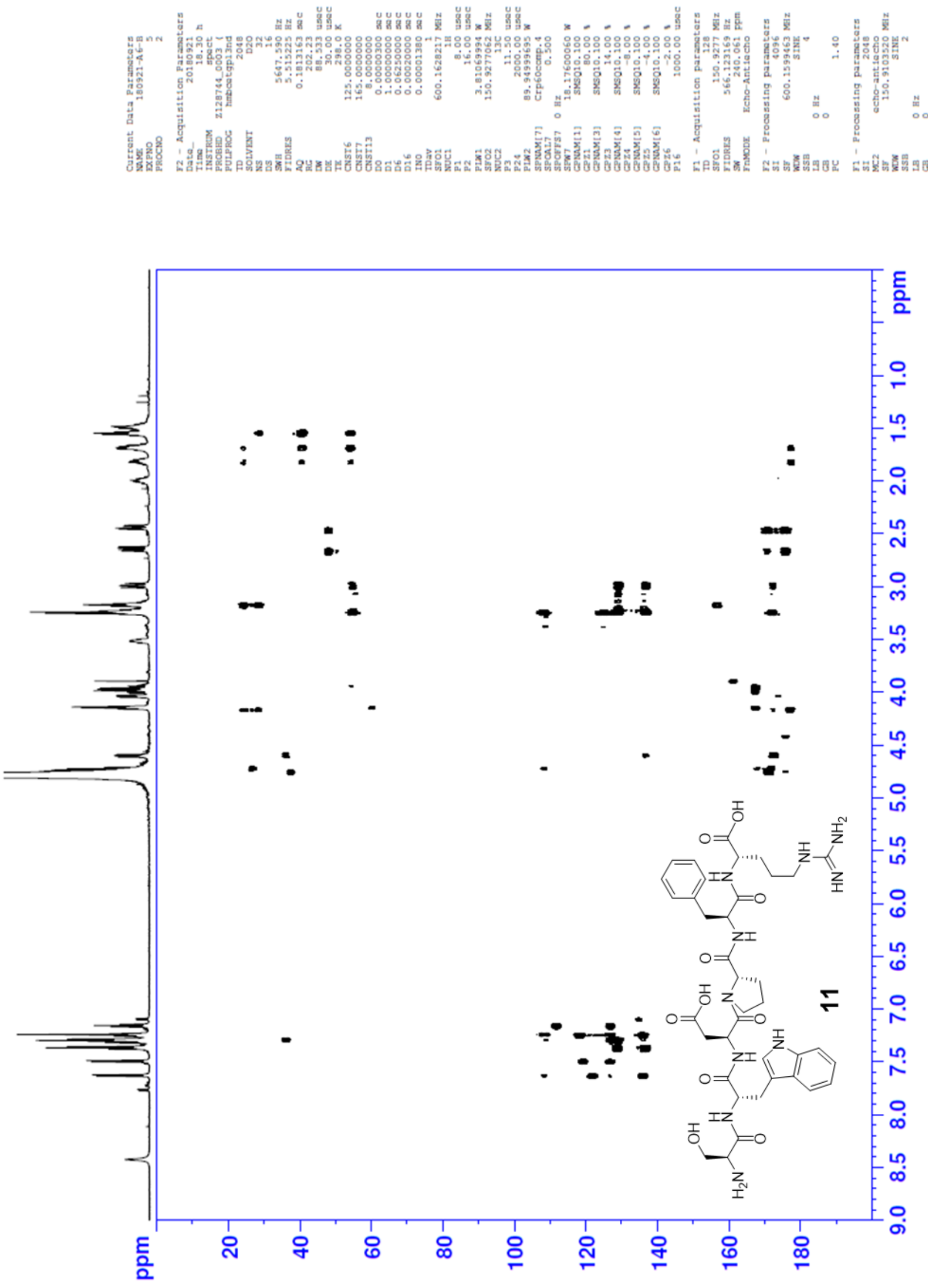


\section{LC/MS of Compound 12}<smiles>N=C(N)NCCC[C@H](NC(=O)[C@H](Cc1ccccc1)NC(=O)[C@@H]1CCC(=O)N1[C@H](CC(=O)O)C(=O)N[C@@H](CC(=O)O)C(=O)O)C(=O)N[C@@H](Cc1c[nH]c2ccccc12)C(=O)N[C@@H](CO)C(=O)O</smiles>

Chemical Formula: $\mathrm{C}_{42} \mathrm{H}_{55} \mathrm{~N}_{11} \mathrm{O}_{13}$

Exact Mass: 921.40

Molecular Weight: 921.97

mAU

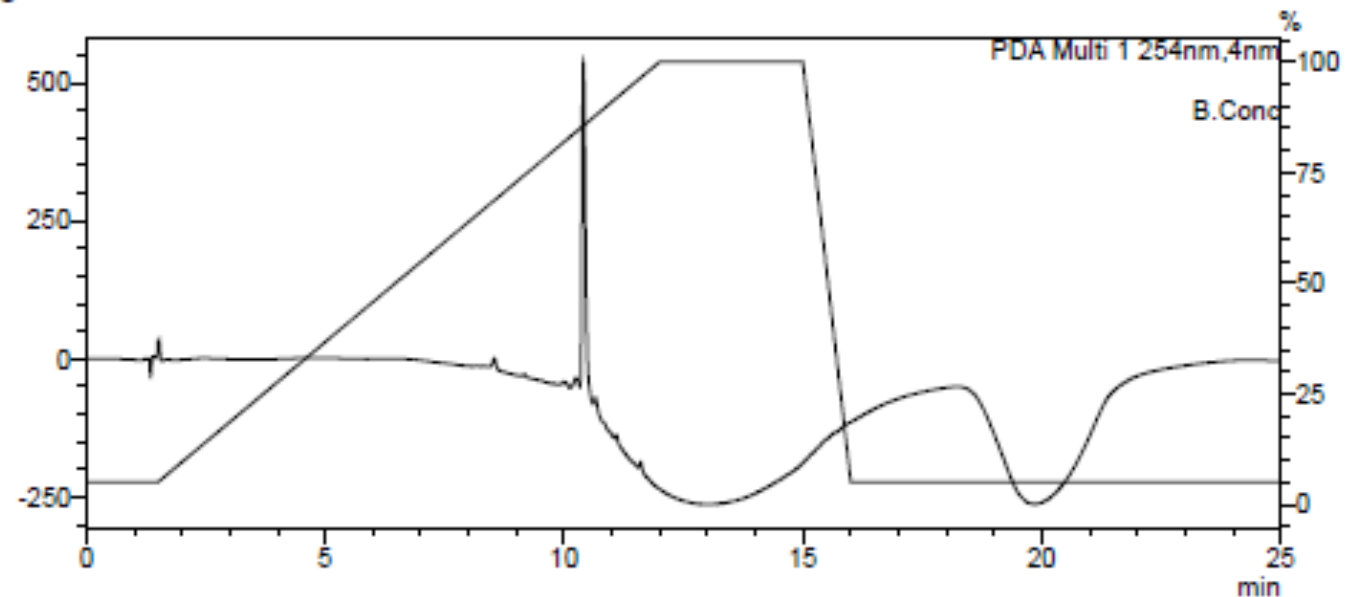

$(x 10,000,000)$

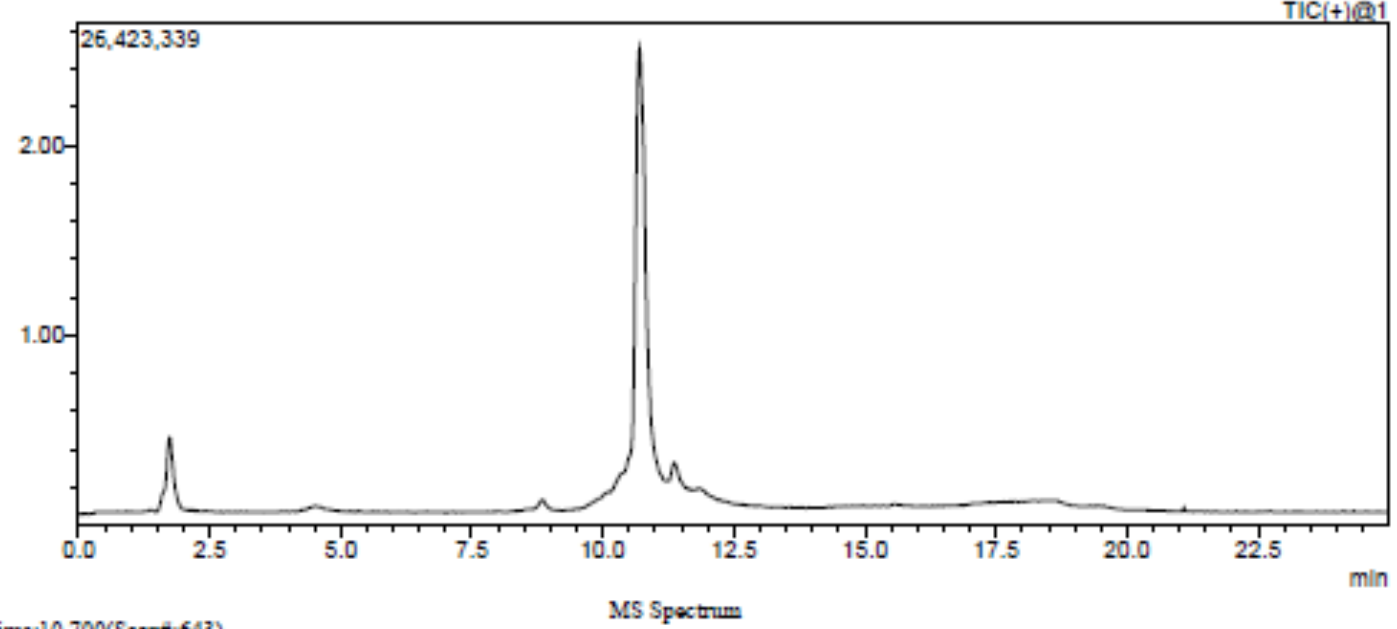

R. Time: 10.700 (Scane 643 )

Sogment 1 - Evont

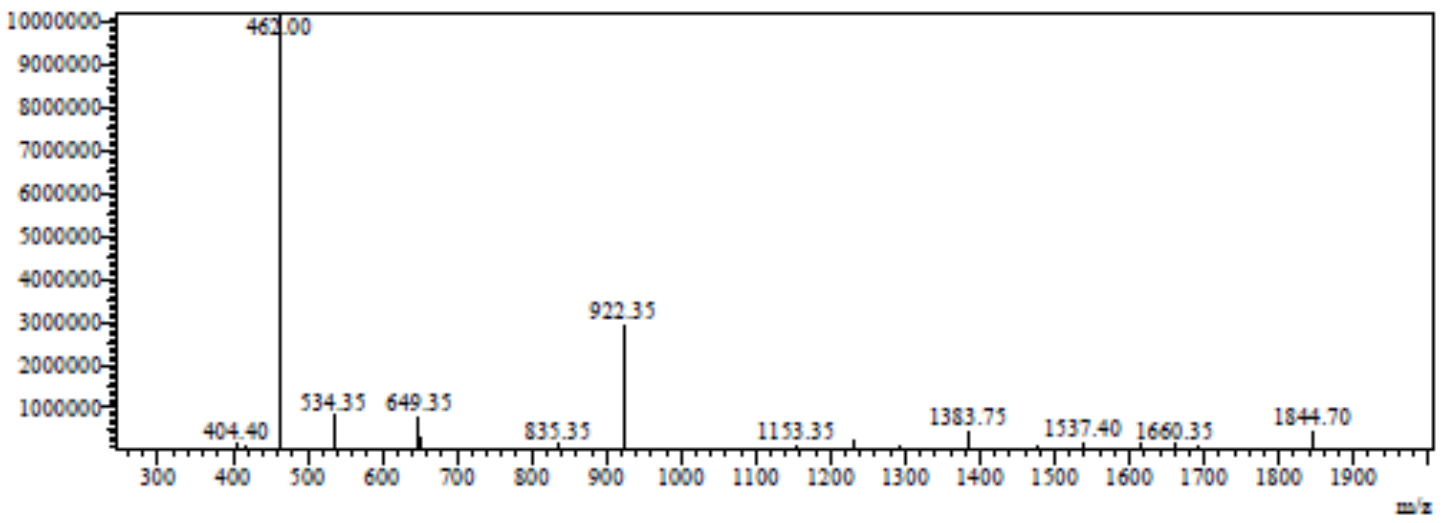




\section{${ }^{1} \mathrm{H}$ NMR of Compound 12}
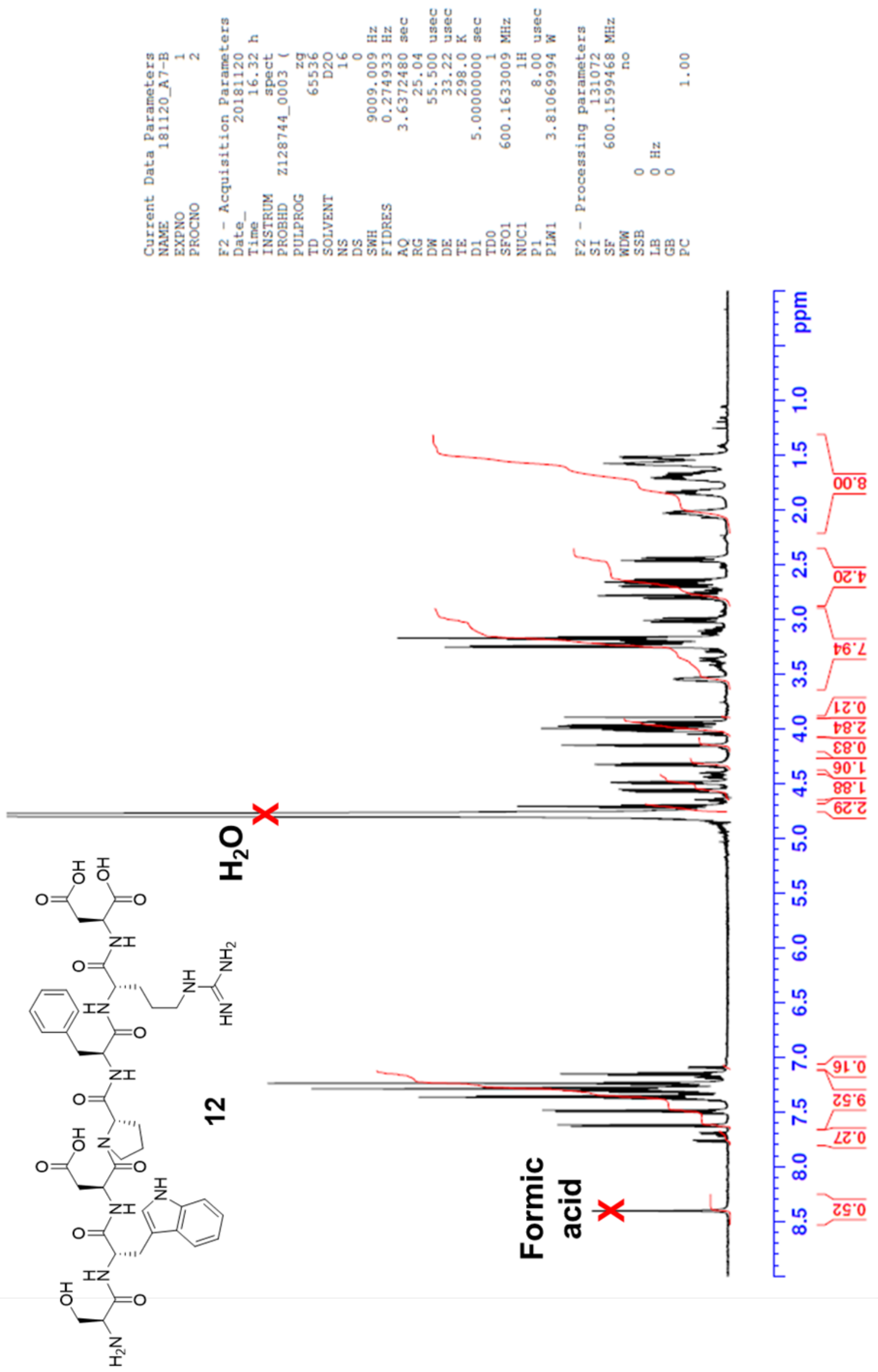


\section{${ }^{1} \mathrm{H}-{ }^{1} \mathrm{H}$ COSY of Compound 12}
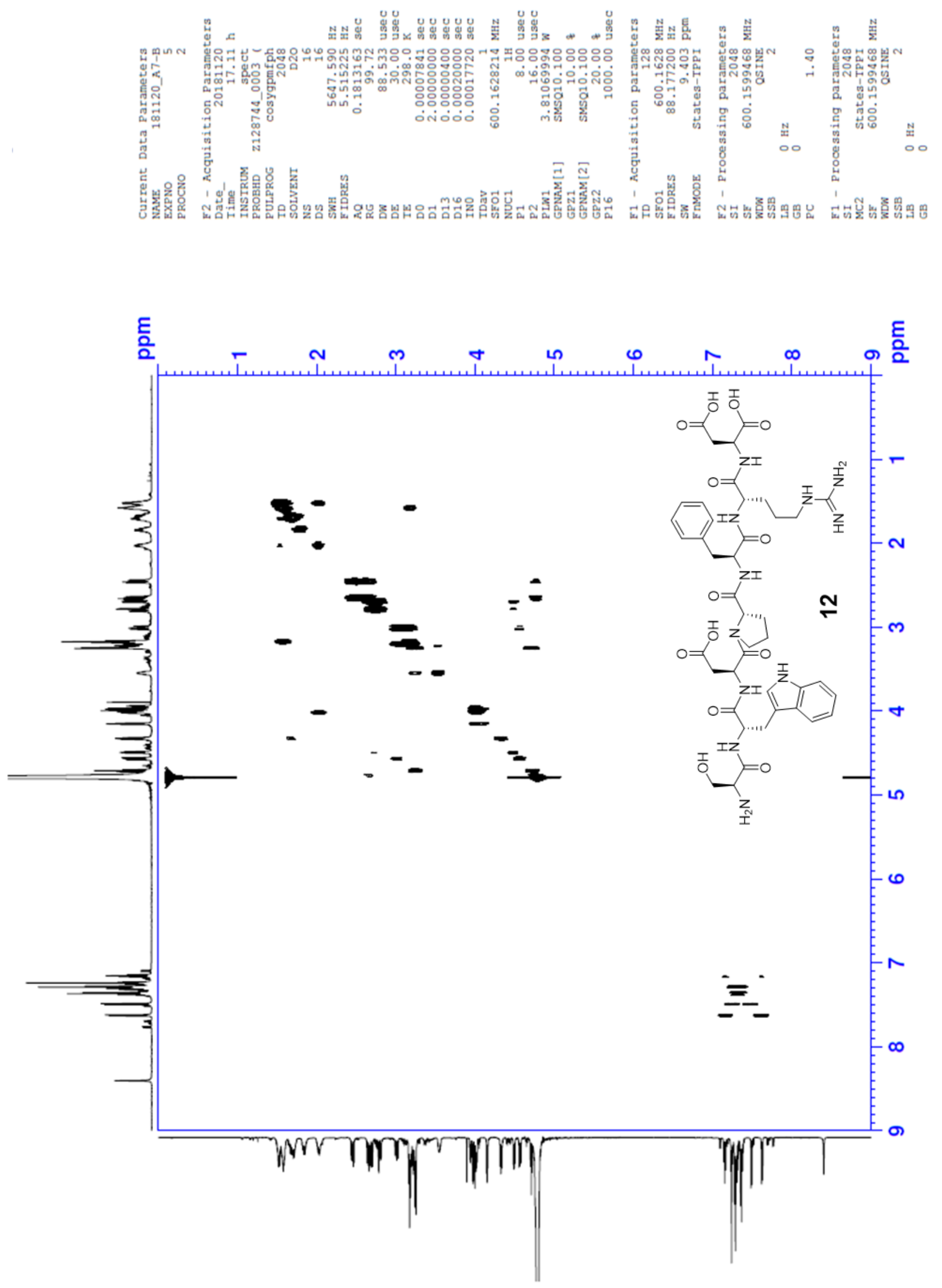


\section{${ }^{1} \mathrm{H}-{ }^{13} \mathrm{C}$ HSQC of Compound 12}
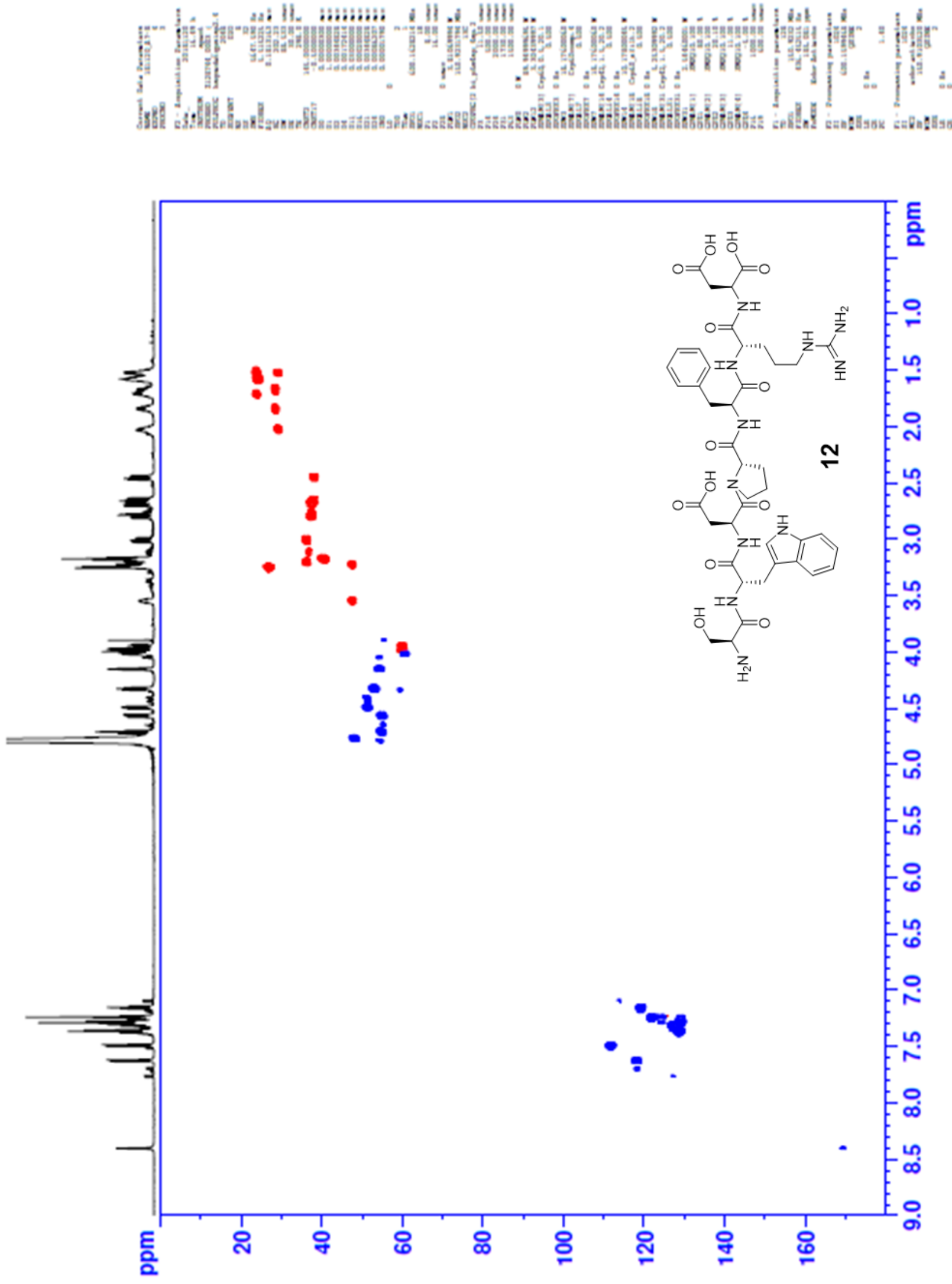


\section{${ }^{1} \mathrm{H}-{ }^{13} \mathrm{C}$ HMBC of Compound 12}
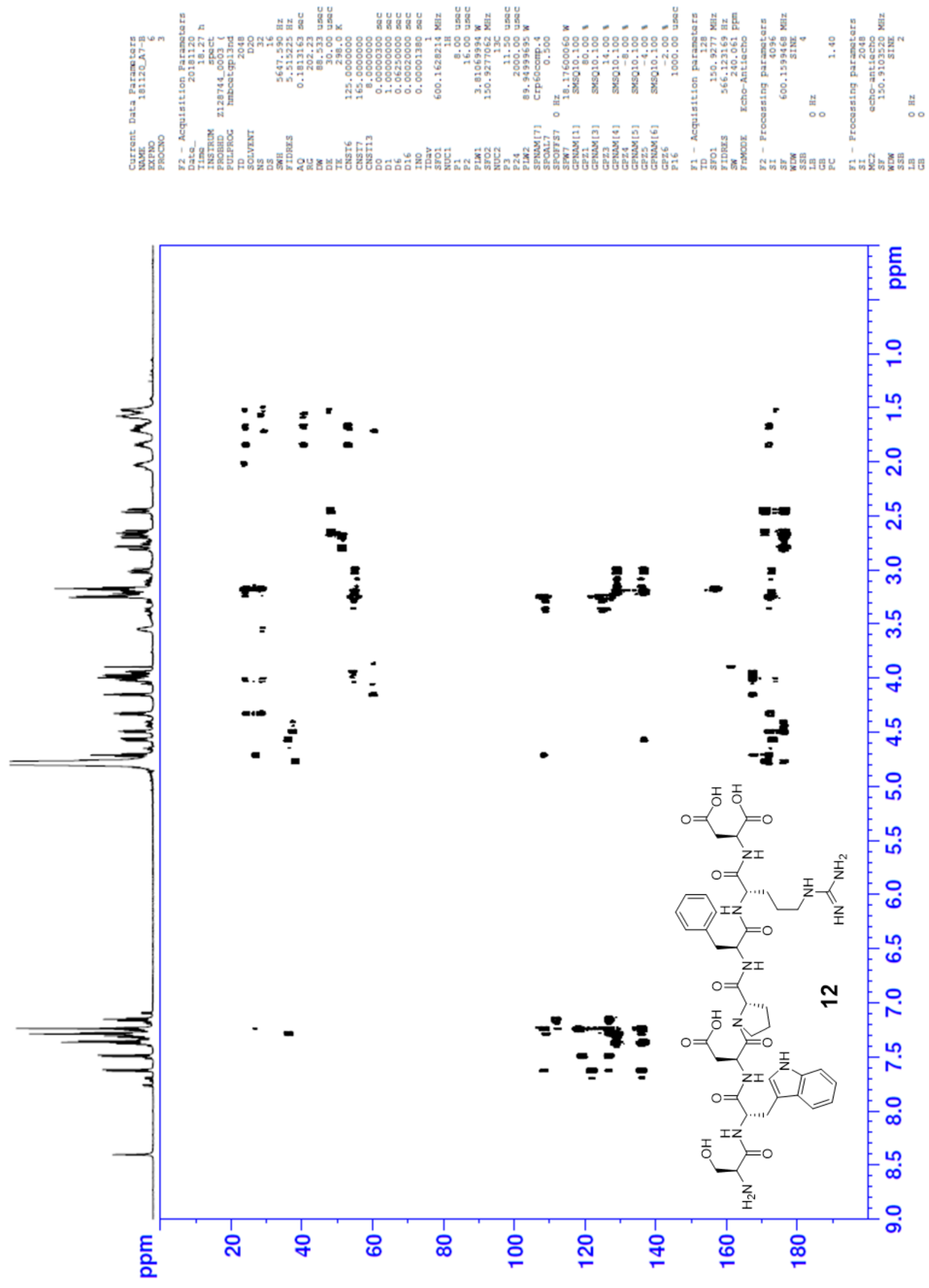


\section{LC/MS of Compound 13}<smiles>N=C(N)NCCC[C@H](NC(=O)C(Cc1ccccc1)NC(=O)[C@H]1CCCN1C(=O)[C@H](CC(=O)O)NC(=O)[C@H](CC(=O)O)NC(=O)[C@H](N)CO)C(=O)N[C@@H](Cc1c[nH]c2ccccc12)C(=O)N[C@@H](Cc1c[nH]c2ccccc12)C(=O)O</smiles>

Chemical Formula: $\mathrm{C}_{53} \mathrm{H}_{65} \mathrm{~N}_{13} \mathrm{O}_{14}$ Exact Mass: 1107.48

Molecular Weight: 1108.18

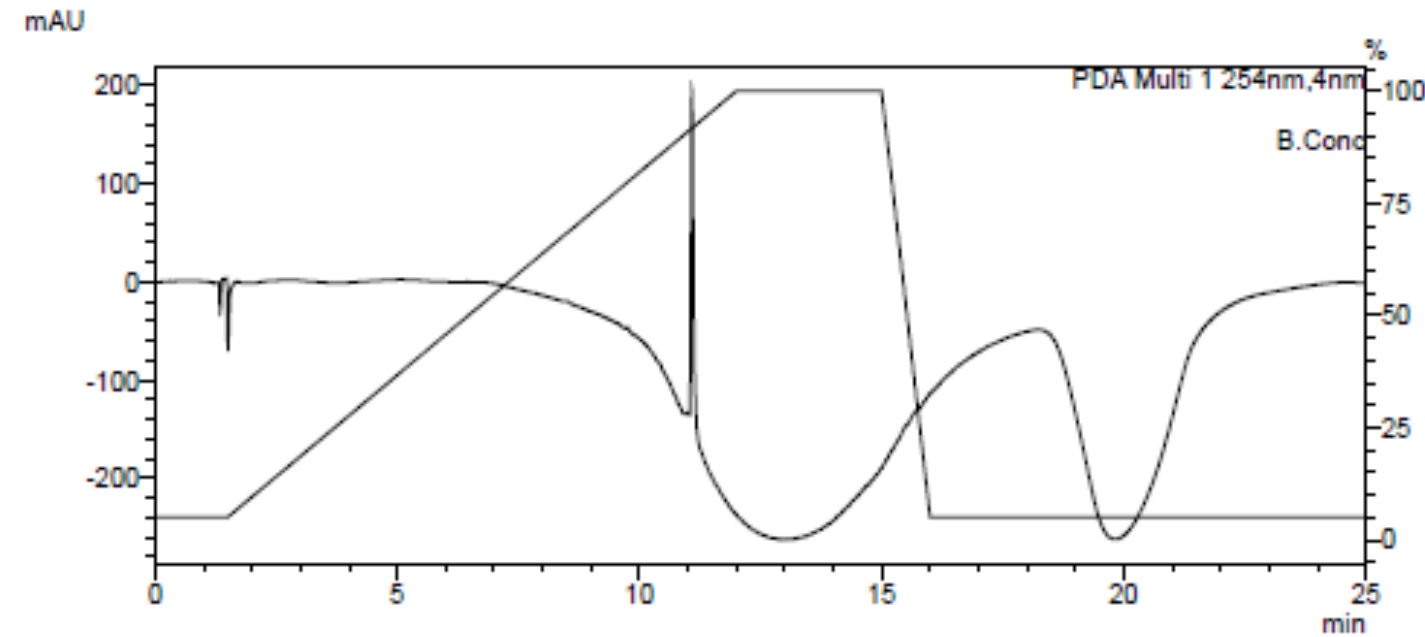

$(x 10,000,000)$

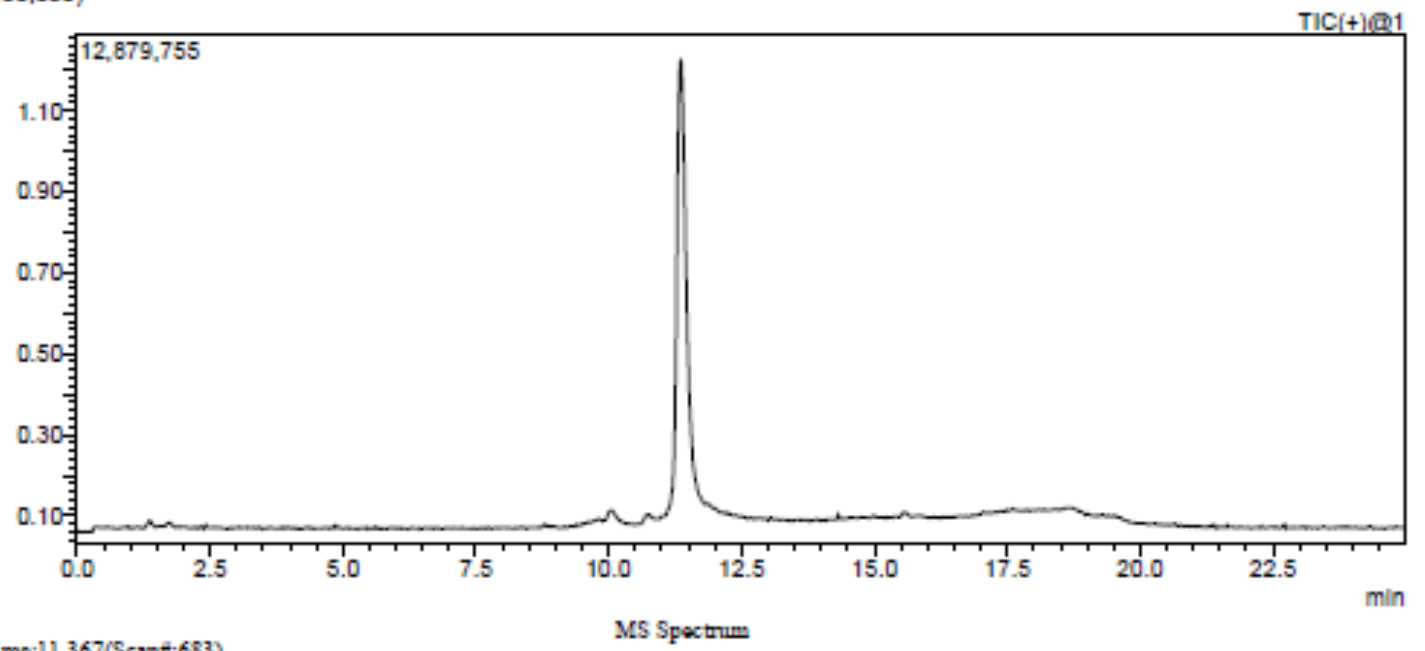

R. Time:11.367(Scane:683)

Sogment 1 - Evout 1

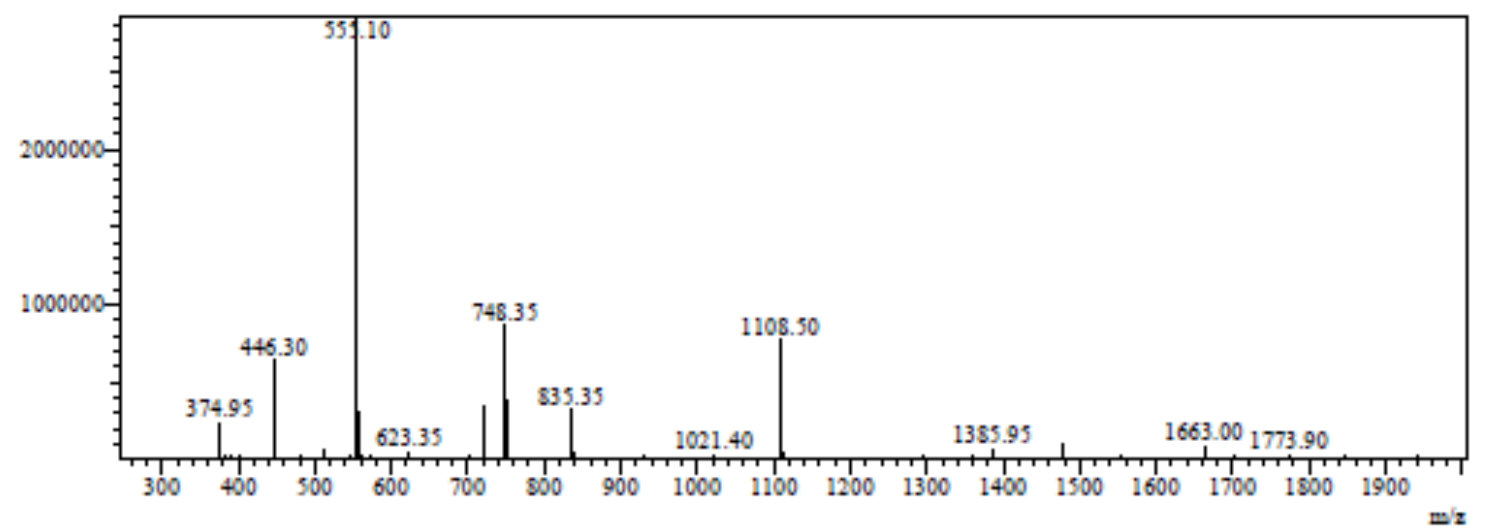




\section{${ }^{1} \mathrm{H}$ NMR of Compound 13}
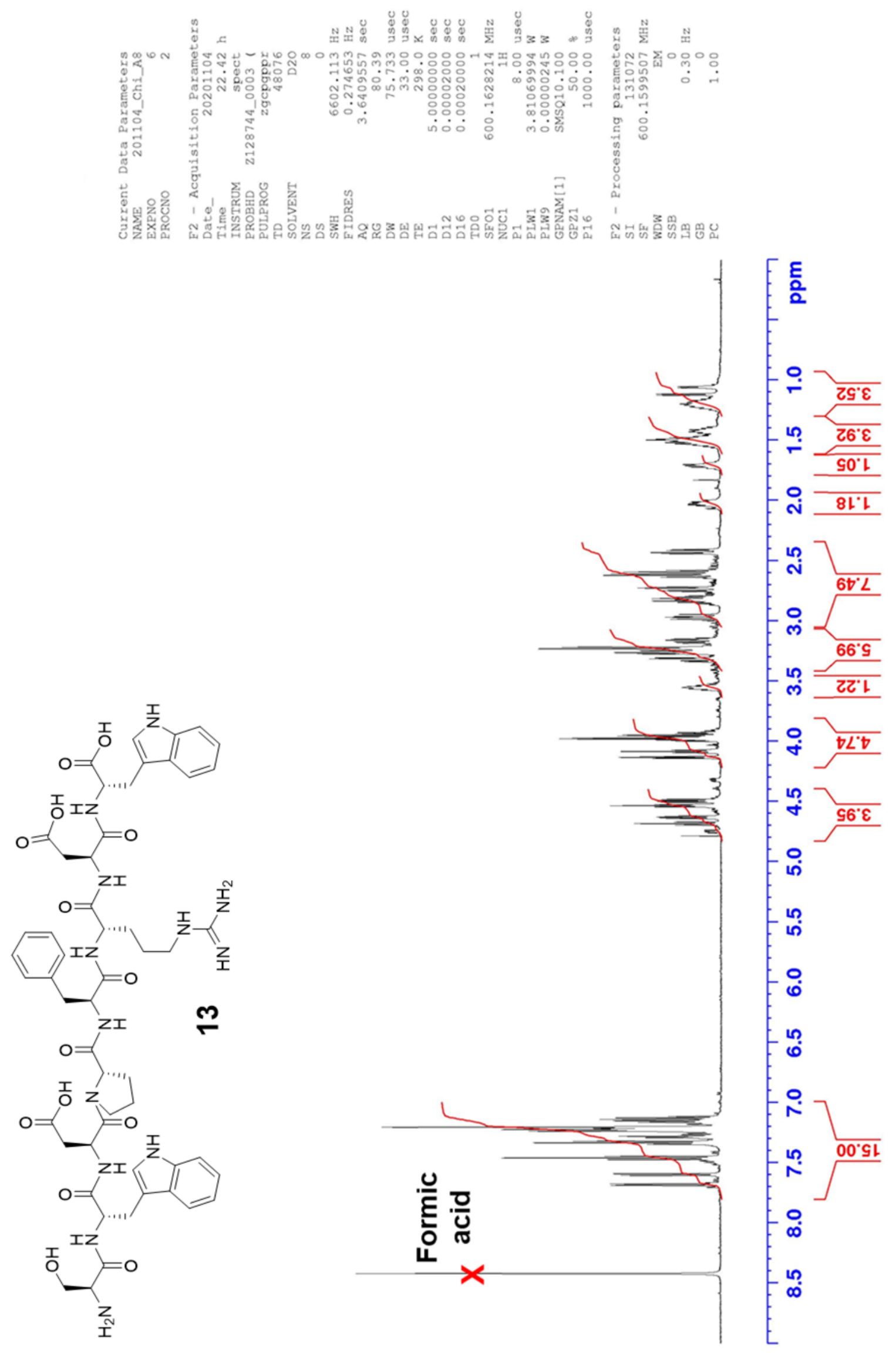


\section{${ }^{1} \mathrm{H}-{ }^{1} \mathrm{H}$ COSY of Compound 13}

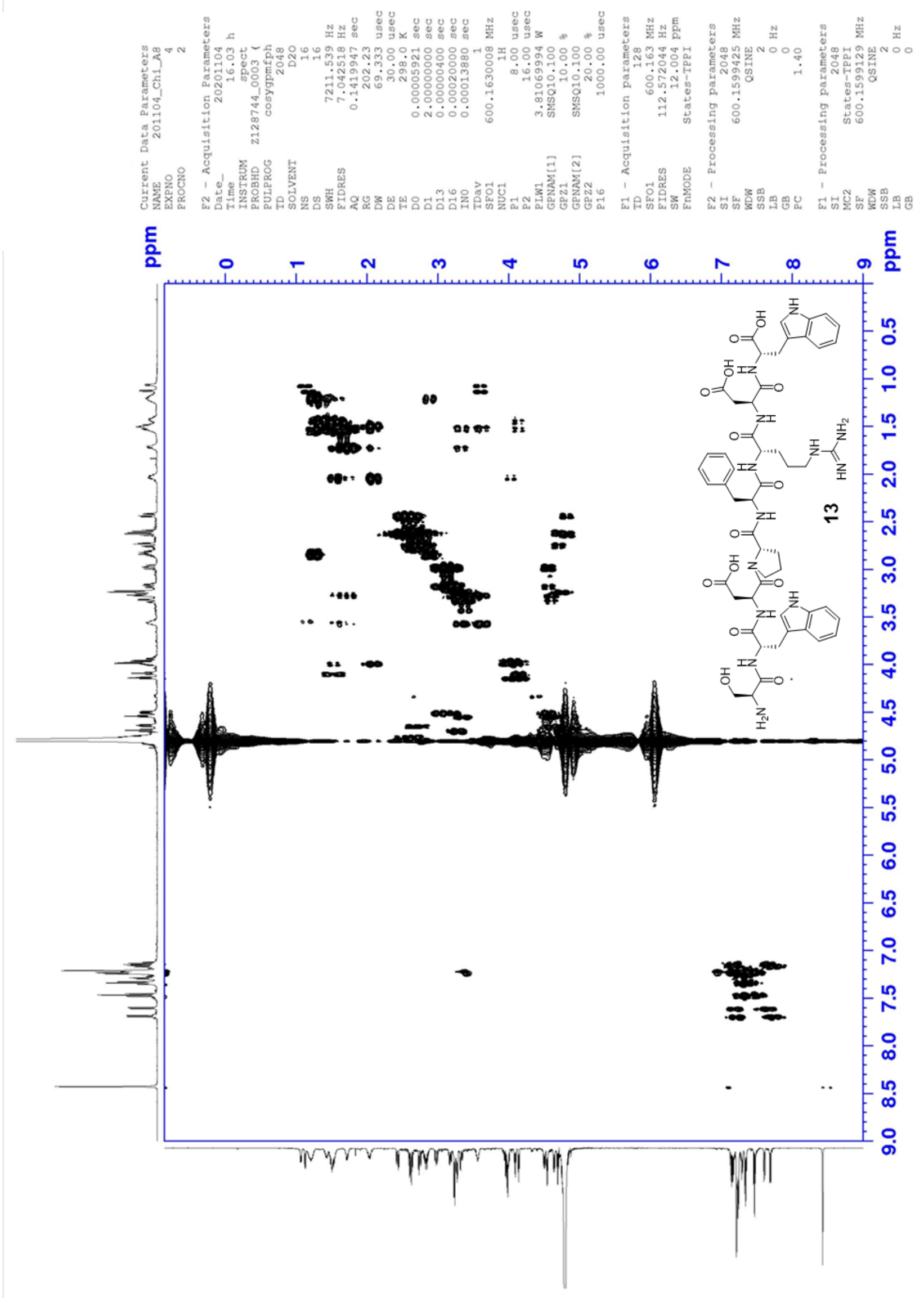




\section{${ }^{1} \mathrm{H}-{ }^{13} \mathrm{C}$ HSQC of Compound 13}

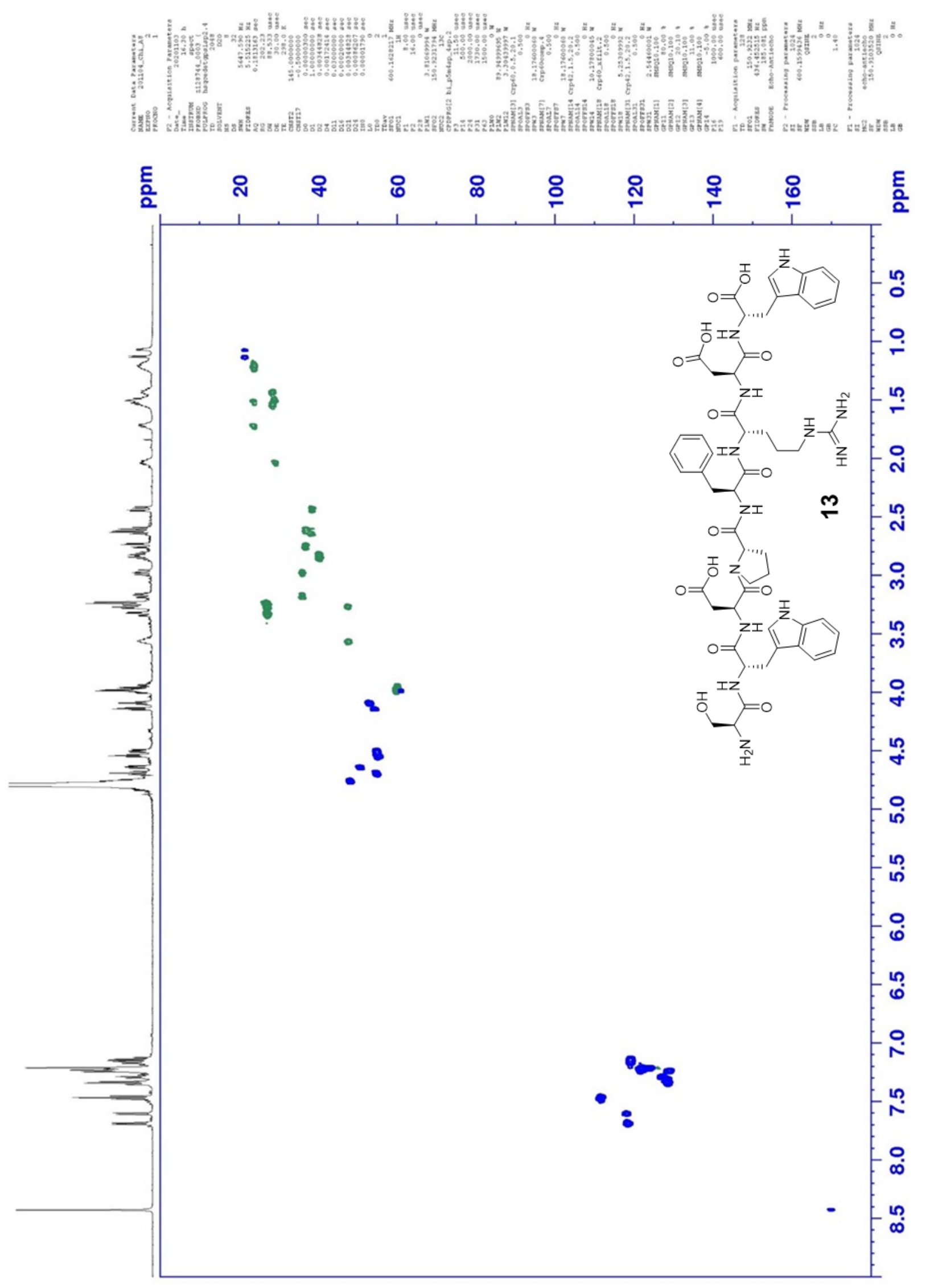




\section{${ }^{1} \mathrm{H}-{ }^{13} \mathrm{C}$ HMBC of Compound 13}

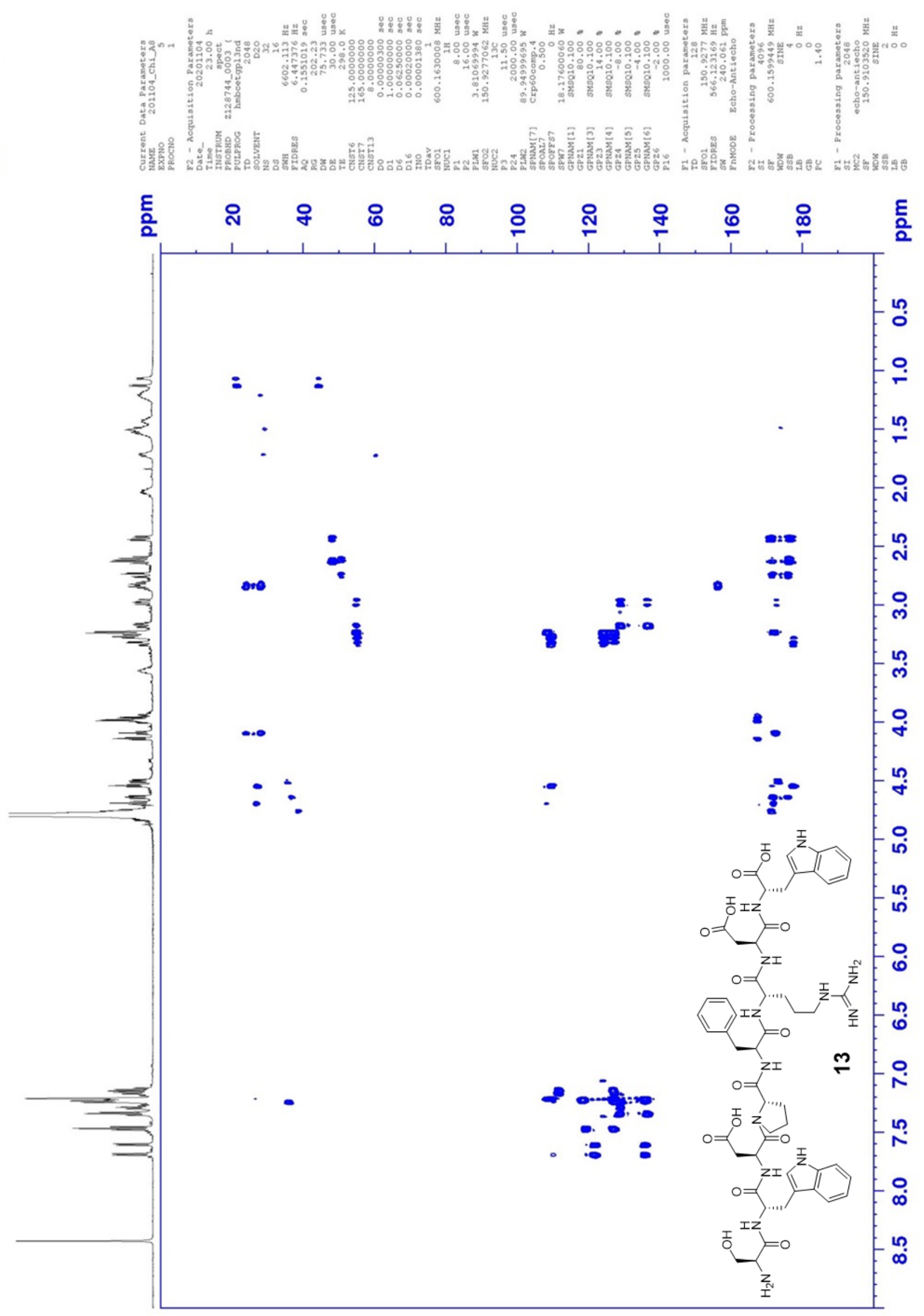

